\title{
Abstracts of the 12th Annual Meeting of Israel Society for Neuroscience
}

Eilat, Israel, December 14 - 16, 2003

Mutation in the glucocerebrosidase gene in Ashkenazi Mutation in the glucocerebrosidase gene
Jewish patients with Parkinson disease

Aharon-PeretzJ., ${ }^{1,4}$, dRosenbaum $H^{2,4}$, Gershoni-Baruch $R^{3,4}$ ${ }^{I}$ Cognitive Neurology and Movement Disorder Unit, ${ }^{2}$ Dept. of Hematology and Bone Marrow Transplantation, ${ }^{3}$ Dept. of Human Genetics, Rambam Medical Center; ${ }^{4}$ Rappaport

Faculty of Medicine; Technion, Haifa

Background: The association of Parkinsonism with type 1 Gaucher disease (GD) has recently been repeatedly reported. The objective of the present study was to evaluate the impact of glucocerebrosidase (GBA) mutations on idiopathic Parkinson disease (PD). Methods: We screened for five GBA mutations (N370S, L444P, 84GG, IVS+1, V394L) a clinic-based cohort of 30 consecutive Ashkenazi patients diagnosed with idiopathic Results: Ten PD patients carried either one or two GD alleles. Five N370S heterozygotes, four $84 \mathrm{GG}$ heterozygotes, and one N370S homozygote were identified. GD carriers did not differ from non-carriers with regard to initial motor manifestations, initial response to levodopa or dopaminergic agonists, development of motor dyskinesias or positive family history of $P D$ in a first or second-degree relative. GD carriers had a significantly younger age of PD onset. All $84 \mathrm{GG}$ carriers had noticeable cognitive deterioration. Conclusions: One in three Askhenazi PD patients carriers a GD mutation. The nature of this remarkable association, reported here for the first time, remains elusive. A possible explanation would involve deficient proteolysis of misfolded proteins deposited and aggregated in excess in dopaminergic neurons. Our result suggests that heterozygosity to a non-neuropathic GD mutation may not be absolutely considered an asymptomatic state.

Amygdalar GABAergic involvement in extinction of conditioned taste aversion and possible relevance to post traumatic stress

Akirav I. and Dudai Y.

Dept. of Neurobiology, Weizmann Institute of Science, Rehovot Experimental extinction is the decline in frequency or intensity of the conditioned response following the withdrawal of reinforcement. It does not reflect forgetting but rather relearning, in which the new association of the conditioned stimulus with the absence of the original reinforcer comes to control behavior. It has been suggested that in post-traumatic stress disorder (PTSD), an anxiety disorder that may develop following exposure to extreme emotional trauma, persistent re-experience of the traumatic event is a consequence of diminished capacity to extinguish emotional conditioned responses. Thus, the initial emotional reaction to the extreme event does not decay properly with time. This may involve use-dependent alterations in inhibitory circuits in the amygdala, a brain area known to control emotional valence and response.

Here, we examined the possible involvement of the $\mathrm{GABA}_{\mathrm{A}}$ (gamma aminobutyric acid) receptors in the basolatera amygdala (BLA) in the acquisition and extinction of conditioned taste aversion (CTA) in the rat. In CTA, the subject learns to associate a taste with delayed malaise. Rats were subjected to CTA training to a novel taste (saccharine) followed by repeated presentation of the conditioned taste in the absence of the malaise-inducing agent. Infusion of a $\mathrm{GABA}_{\mathrm{A}}$ agonist (muscimol, $0.05 \mu \mathrm{g} / \mu \mathrm{l}$ ) into the amygdala following retrieval in an extinction mode blocked extinction of CTA. In contrast, infusion of a $\mathrm{GABA}_{\mathrm{A}}$ antagonis (Bicuculline, $0.005 \mu \mathrm{g} / \mu \mathrm{l}$ ) into the amygdala facilitated CTA acquisition without a significant effect on extinction. The data indicate that GABAergic neurotransmission plays a role in extinction, and suggest that GABAergic mechanisms may contribute to pathological emotional response following trauma.

Supported by the Israeli Science Foundation
Genetic correlational analysis of glycogen synthase kinase- 3 and prepulse inhibition in inbred mice Amar S. ${ }^{1}$, Jones B.C. ${ }^{2}$, Belmaker $\mathrm{RH}^{1}$ and Agam G. ${ }^{1}$ ${ }^{i}$ Stanley Foundation Research Center and Dept. of Clinical Biochemistry, Faculty of Health Sciences, Ben Gurion University of the Negev, Beersheva. Israel. ${ }^{2}$ Dept. of

Biobehavioral Health, The Pennsylvania State University, University Park, PA. USA

One of the key characteristics of schizophrenia is difficulty with sensorimotor gating. Thus, those afflicted are less able to shut out non-consequential stimuli that others are able to filter out as "noise". Prepulse inhibition (PPI) is a diagnostic method by which the amplitude of an elicited reflex (e.g. acoustic or tactile startle, eyeblink etc.) can be dampened by another stimulus delivered immediately before the reflex-eliciting stimulus (prepulse). Unafflicted individuals show substantial prepulse dampening of the reflex; however, those diagnosed with schizophrenia produce the reflex with diminished prepulse inhibition. PPI can also be measured in animals and in rodents it shows genetic-based variability in expression. The enzyme glycogen synthase kinase 3 (GSK-3) has also been shown to have decreased activity in the frontal cortex of schizophrenics. The question then is whether GSK-3 is merely a marker for schizophrenia or is there a functional relationship? In order to address this question, we sought to start by examining the association between PPI and GSK-3 in genetically defined mice. In this way we can capture the genetic variability in these two parameters without the necessity of measuring both in the same animals. We searched the literature for PPI in inbred mice (Crawley, et al., Psychopharmacology 132: 107-124, 1997), ordered the same 10 strains from the supplier and measured GSK-3 $\beta$ protein levels and total GSK-3 $(\alpha+\beta)$ activity in the frontal cortex. Genetic correlational analysis, using the Pearson product-moment coefficient revealed statistically significant associations between GSK-3 activity and acoustic-pulsed tactile startle PPI $(r=0.779, p<0.005)$ and acoustic-pulsed acoustic startle $(r=0.727, p<0.01)$. Abundance of the enzyme protein was not significantly correlated with PPI of either method. These preliminary results showing enzyme activity to be related to a sensorimotor gating function is consistent with GSK-3 having a functional role in neurobiology. Thus, low expression of the enzyme and PPI may point to neuropathology related to schizophrenia.

Nerve epithelial interaction in chemical ocular injury: Morphological and biochemical study

Amir A., Fishbine E., Cohen M., Dachir S., Gutman H., Shalem Y., Cohen L., Sahar R. and Kadar T.

Dept. of Pharmacology, Israel Institute for Biological Research, Ness Ziona.

The cornea is a highly innervated tissue, richly supplied by sensory nerve fibers deriving from the ophthalmic division of the trigeminal nerve. These sensory fibers exert important trophic influences on the corneal epithelium and contribute to the maintenance of a healthy ocular surface. Chemical trauma to the eye stimulates ocular nerve fibers and plays a role in the acute neurogenic inflammation mediated mostly by substance $P$ and CGRP

The possible involvement of sensory nerve damage in the pathogenesis of chemical ocular injury, rabbit eyes were exposed to sulfur mustard (SM) vapor. A clinical follow-up was carried out for up to 3 months. Blinking response, using a Cochet-Bonnet esthesiometer, tested corneal sensitivity. Animals were sacrificed at different time points, eyes enucleated and frozen. The morphology of corneal nerves was visualized in whole mount corneal preparations stained by cholinesterase histochemistry. Biochemical analysis of neuromediators was conducted in aqueous humour $(\mathrm{AQ})$ and cornea for detection of neurogenic inflammation

Initial changes in CGRP content of $A Q$ and cornea were measured within the first $24 \mathrm{hrs}$ after exposure. Corneal erosions (epithelial loss) were observed during the first 3 days after exposure and then healed spontaneously. During this time and for weeks afterwards, corneal nerves underwent a slow retrograde degeneration, concomitant with alterations in 
corneal sensitivity. Following a latent period, $50-90 \%$ of corneas displayed delayed clinical symptoms consisting of blood vessels ingrowth and recurrent corneal erosions. Regeneration of nerve fibers took place, if at all, months after exposure. It is suggested that the impairment in corneal innervation following SM exposure contributes to the development of prolonged corneal injuries through the lack of neurotrophic mediators.

Supported in part by the U.S. Army Medical Research and

Material Command under contract No. DAMDI7 -03-C-0064.

Zebrafish serotonin- $\mathrm{N}$-acetyltransferase-2 gene regulation: Pineal-restrictive downstream module consisting of a functional E-box and PCE

Appelbaum L. ${ }^{1}$. and Gothilf Y. ${ }^{2}$

${ }^{T}$ Faculty of life Sciences, Bar Ilan University Ramat-Gan:

${ }^{2}$ Faculty of Life Sciences, Tel Aviv University, Tel Aviv

The expression of a battery of specific genes in the pineal gland is mandatory for proper transduction of photoperiodic information and rhythmic melatonin secretion by the pineal gland. Serotonin- $N$-acetyltransferase (AANAT), the key enzyme in the melatonin synthesis is specifically expressed in the pineal gland and retinal photoreceptors in all vertebrates. In vivo transient expression analyses of promoter-reporter constructs, in which fluorescent proteins are driven by regulatory regions of the zebrafish (zf) AANAT-2 gene, indicated that pineal-specific expression of this gene requires a $257 \mathrm{bp}$ fragment, located more than $6 \mathrm{~kb}$ downstream of the structural gene. This fragment was designated PRDM in the context of the zfAANAT-2 promoter and heterologous promoters indicated that this module serves two functions: One is the enhancement of promoter activity in the pineal and the second is inhibition of promoter activity in non-pineal tissues. The former is an autonomic property of PRDM while the later is accomplished by cooperation with the upstream regulatory region of the zfAANAT-2 gene. Functional analyses of the PRDM sequence revealed that three photoreceptor conserved elements (PCE, TAATC) and a single perfect E-box (CACGTG) are crucial for PRDM function. These results propose a new mechanism for pineal-specificity that includes a novel function and interaction of downstream E-box and PCEs.

The lipophilic transition metal chelator DP-b99 modulates intracellular zinc

Arielly R. ${ }^{1}$, Friedman J.E. ${ }^{2}$, Hershfinkel M. ${ }^{1}$, Moran A. ${ }^{1}$

Sekler I.', Shapiro 1.2, Kozak A. and Angel I.

Physiology, Ben Gurion University of the Negev, Beer Sheva; ${ }^{2}$ D-Pharm Ltd, Rehovot

Zinc plays an essential role in mammalian devclopment and function. Following brain ischemia or injury, massive amounts of zinc are released into the extracellular milieu. This zinc can then enter neurons via various routes including AMPA and calcium channels. The result is a rapid elevation in free intracellular zinc activating pathways leading to cell injury and death. Thus, maintenance of normal intracellular $\mathrm{Zn}^{2+}$ levels while preventing a massive increase during periods of stress must be achieved and would be beneficial in preventing zinc-induced neurodegeneration. A family of lipophilic analogues of BAPTA, which exerts its activities in the membrane region is described. A member of this family, DP-b99, preferentially chelates $\mathrm{Zn}^{2+}$ and $\mathrm{Cu}^{2+}$ as compared to calcium. Recently DP-b99 has successfully completed a Phase Ila stroke study. The present study tested whether DP-699 can reduce excess free zinc levels in the intracellular environment. Min- 6 cells were loaded with the fluorescent zinc probe $\mathrm{Zn}-2 \mathrm{~F}$ DA and monitored using fluorescence microscopy. An influx of Zinc $(100 \mu \mathrm{M})$ was stimulated by potassium-induced depolarization, demonstrated by increased fluorescence. Subsequent addition of DP-b99 resulted in a decrease in fluorescence, showing that DP-b99 chelates cytoplasmic zinc and reduces its free intracellular concentration. This occurred in both the absence and presence of extracellular calcium. The rate of drop in fluorescence correlated with the concentration of DP-b99 (10-50 $\mu \mathrm{M})$ used. Additional studies examining zinc toxicity in primary cortical neurons found that $20 \mu \mathrm{M}$ DP-b99 reduced zinc-induced toxicity by $50 \%$. We suggest that the efficacy of DP-b99 could be due to its ability to chelate excess zinc and help restore zinc homeostasis to neurons. This ability to modulate zinc should be beneficial in the treatment of neurological degenerative disorders such as Alzheimer's disease

Support Contributed By D-Pharm Ltd
Isolation and characterization of neuronal stem cell progenitors from human umbilical cord blood (UCB) for

putative use in cell therapy 'Dept. of Pharmacology, School of Pharmacy, The Hebrew University of Jerusalem. ${ }^{2}$ Dept. of Hematology and Bone Marrow Transplantation ${ }^{2}$, Chaim Sheba Medical Center. TelHashomer

Neuroprotection towards neurodegenerative disorders involves modern technologies of cell and gene therapies. The rate-limiting factor in these technologies is the lack of neurona tissue for transplantation. Recent reports suggested that non-hematopoietic progenitors deriving from umbilical cord blood (UCB) may differentiate into neuronal cells in vitro [1] and in vivo [2]. Therefore, we further developing a technology for future use of neuronal progenitors of UCB origin for therapy of central nervous system disorders. In the present study we have cultured (on collagen coated plates) non-hematopoietic precursors from mononuclear cells (MNC) of UCB. These MNC progenitors were highly adherent to collagen similarly to control neuronal cell lines as pheochromocytoma $\mathrm{PCl} 2$ and neuroblastoma. During 14 days of culture using unique conditioning combinations, we found populations of progenitors that survive, proliferate and generate a neuronal-like morphological phenotype (cells showing "neurite" outgrowths of different length). We have further characterized these neuronal-like progenitor cells using immunofluorescent antibodies against different neuronal markers. Some of progenitor populations were specifically stained with anti trk-A anti microtubule-associated-protein 2 (MAP-2), and anti nestin. Additional neuronal markers are evaluated by PCR reactions and immunofluorescence. Some of the neuronal progenitors were cloned and we attempt to develop optimal conditions for their proliferation and differentiation. As these ncuronal progenitors were positively immuno-stained to the nerve growth factor (NGF)-trk-A receptor, experiments are performed to investigate the cellular effects of $N G F$ on $U C B$ progenitor fate. Our main rational goal is to cstablish, characterize and optimize cell culture conditions for the proliferation and differentiation of UCB neuronal progenitors that will be of therapeutic relevance in transplantation against neurodegenerative disorders.

[1]. Bicknese AR, Henderson V, Sinclair-Goodwin HS, et al. (2001) Cell derived from human fetal cord blood express markers for neurons, astrocytes and oligodendrocytes. Exp. Neurol. 170, 199; [2]. Zigova T, Song \$, Willing AE, et al (2002) Human umbilical cord blood cells express neural antigens after transplantation into the developing rat brain. Cell Trans. 11, 265-274.

Estimation of the tension in deviated white matter tracts caused by brain lesions measured by diffusion tensor

imaging ${ }_{1,2}$, Pianka $P{ }^{1}{ }^{1}$, Rotshtein $P .{ }^{1}$, Sigal M. ${ }^{1}$ and Hendler $T .{ }^{1}$ IFunctional Brain Imaging Unit. Tel Aviv Sourasky Medical Center; ${ }^{2}$ School of Chemistry. Tel Aviv University. Tel Aviv

Diffusion Tensor Imaging (DTI) is used to estimate the tension of deviated white matter caused by brain lesions. DTI is a recent development in MRI that is used for specific white matter mapping. It is based on the unique diffusion properties of water in white matter. While parallel to neuronal fibers the diffusion is relatively free, perpendicular to them it is hindered. This diffusion anisotropy is measured by DTI and quantified by the fractional anisotropy index. In addition, DTI provides the architecture and 3D alignment of the fibers. DTI was recently shown to be very helpful in delineating white matter both within brain lesions and surrounding them. Displacement of white matter fibers may be one of the consequences of tumor growth adjacent to white matter and may lead to significant disability to the patient. We found that the fractional anisotropy (FA) is increased by $38 \%$ in areas of shifted white matter fibers. By contrast, the trace apparent diffusion coefficient values in those areas were found to be similar to normal control values. Analysis of the three diffusion eigenvalues revealed that the increase in the fractional anisotropy is a result of two processes. The first is the increase in the diffusion parallel to the fibers - lambdal (by $18 \%$ ) and the second is the decrease in the diffusion perpendicular to fibers - lambda3 (by 34\%) as compared with the contralateral side. These opposing changes cause an increase in the diffusion anisotropy but no change in the trace $A D C$. It is suggested that the pressure caused by the lesion may lead to an increase in white matter fiber tension thus causing an increase in lambdal. On the other hand, the same pressure causes increased fiber density per unit area leading to a higher degree of restricted diffusion in the extra-cellular space and hence a reduction in lambda3. We propose a new image index given by the ratio lambda3 / lambdal that seem to be highly sensitive to tension in white matter. 
The voltage gated $\mathrm{Ca}^{2+}$ channel is modified by syntaxin $1 \mathrm{~A}$ in a cooperative manner

Atlas D.

Dept. of Biological Chemistry, Inst. of Life Science The

Hebrew University of Jerusalem, Jerusalem 91904

Syntaxin 1A (Sx1A) modifies the activity of voltage-gated $\mathrm{Ca}^{2+}$ channels acting via the cytosolic and the two vicinal transmembrane (TMD) cysteines $(271,272)$ (Cohen et al, 2003; Trus et al., 2001). Here we show that Sx1A modulates the Lc-type $\mathrm{Ca}^{2+}$ channel, $\mathrm{Ca}_{v} 1.2$, in a cooperative manner and explore whether channel clustering or $\mathrm{Sx1A}$ homodimer is responsible for this activity. SxIA formed homodimers, but when mutated at the two vicinal TMD cysteines, was unable to either dimerize or modify the channel activity. Also application of a vicinal thiol-reagent, phenyl arsine oxide (PAO), abolished SxlA action indicating the accessibility of Cys271, 272 thiols (Arien et al., 2003). However, the supralinear mode of channel inhibition was correlated to the monomeric form of Sx1A and was apparent only when the three-channel subunits $\alpha_{1} 1.2 / \alpha 2 \delta 1 / \beta 2 \mathrm{a}$ were present. This functional demonstration of cooperativity suggests that the three-subunit channel responds as a cluster and SxIA monomers bind to a dimer (or more) of a three-subunit $\mathrm{Ca}^{2+}$ channel. The rapid transduction of a channel conformational change, driven by $\mathrm{Ca}^{2+}$ entry to the exocytotic machinery, is consistent with the formation of a channel/Sx1A cluster. This non-linear relationship could convey the co-operativity that is the hallmark of the process of neurotransmitter release.

Arien et al., J Biol Chem. (2003) 278(31): 29231-9; Cohen R, Elferink LA, Atlas D. J Biol Chem. (2003) 278(11): 9258-66. Wiser, O., Cohen, R. and Atlas, D. Proc Natl Acad Sci U S A 99 (6): 3968-3973 (2002).

A novel therapeutic vaccination for protection against toxicity of aggregated $\beta$-amyloid in the central nervous system

Avidan H., Butovsky O., Kipnis J., Schori H. and Schwartz M. Neurobiology, Weizmann Institute, Rehovot

Alzheimer's disease is a devastating neurodegenerative disorder in which extracellular deposits of fibrillar $\beta$-amyloid form plaques in the brain. Using a mouse model of neuronal loss induced by local injection of aged (aggregated) $\beta$-amyloid peptide, we show that the physiological ability to fight off the toxic effects of aggregated $\beta$-amyloid is T-cell dependent varies among strains, is suppressed by naturally occurring CD4+CD25+ regulatory $T$ cells, and is amenable to boosting by vaccination with an immunodominant antigen residing in the site of toxicity or with a synthetic antigen that cross-reacts with such self-antigens. Vaccination with the $\beta$-amyloid peptide was not protective. Ex-vivo experiments using mice hippocampal slices showed that microglia become cytotoxic on encountering aggregated $\beta$-amyloid but protective when they encounter a T cell-derived cytokine such as interferon (IFN)- $\gamma$. These findings suggest that immunodominant self-antigens residing in the site of plaque formation, or nonself-antigen that weakly cross-reacts with the relevant self-antigens, should be considered as potential T cell-based therapeutic vaccines for neurodegenerative conditions.

Local circuit alterations in rat model for PTSD Avital A. and Richter-Levin G.

Dept. of Psychology and The Brain \& Behavior Research Center, University of Haifa, Haifa 31905

We have recently shown that exposure of rats to juvenile + adulthood stress results in long-term behavioral symptoms. This behavioral protocol was suggested as a rat model for PTSD.

Previous studies have demonstrated that stress impairs LTP Several years ago we have demonstrated that High-Frequency Stimulation (HFS) to the perforant pathway (PP), which induces LTP in the Dentate Gyrus (DG), results in alterations also in local circuit activity.

The present study was aimed to assess whether in addition to affecting the ability to induce LTP, the exposure to juvenile + adulthood stress would also affect local circuit activity and plasticity.

Rats were exposed to the combination of juvenile and adulthood stress and $1 \mathrm{hr}$ post-adulthood stress were prepared for acute recording of field potentials in response to PP stimulation. In the COMM group, an additional stimulating electrode was positioned in the contra-lateral DG to activate the commissural pathway. Local circuit was examined by applying two stimulating protocols: the paired-pulse stimulation and the commissural stimulation protocols.

The results showed that the exposure to juvenile + adulthood stress was associated with suppression in the ability to induce LTP. In addition, juvenile + adulthood stress was found to be associated with a significant increase in paired-pulse (presumably feed-back) and commissural (presumably feed-forward) -induced inhibition.

HFS, which failed to induce long-lasting LTP, significantly reversed feed-back but not feed-forward inhibition. These results suggest that the exposure to juvenile + adulthood stress is associated with specific impairments in local circuit activity and plasticity. These impairments may be of relevance to our understanding of stress-related disorders such as PTSD.

Supported by a grant from The Israel Foundations Trustees (2000) to G.R.L

Neuronal synchronization and the transmission of sensory information in visual cortical neurons

Azouz R.

Dept. of Physiology, Faculty of Health Sciences, Ben-Gurion University, Beer-Sheva 84105

Neuronal synchronization has been proposed as the mechanism underlying the transient formation of cell assemblies. Although abundant evidence indicates that synchronization is present in many brain regions, its role in sensory information processing is still controversial. Using intracellular recordings from visual cortex in vivo, we find that the generation of action potentials in response to visual stimuli is associated predominantly with transient depolarization of the membrane potential. Cellular conductance measurements suggest that these transient depolarizations arise from the temporal interplay between synchronous excitatory and inhibitory synaptic inputs anverging onto cortical neurons. The magnitude and time course of these depolarizations are influenced by voltage-gated conductances and are tightly tuned for stimulus orientation. Finally, using numerical simulations we show that adaptive spike threshold mechanism dynamically maintains the temporal correlation between cellular input and output. Together these findings suggest that neuronal synchronization plays a critical role in the processing and transmission of sensory information in the visual cortex.

The amygdalar circuits that subserve consolidation of taste memory are not essential for its reconsolidation

Bahar A. Dorfman N. and Dudai Y.

Dept. of Neurobiology, The Weizmann Institute of Science Rehovot.

Even though evidence now exists for the occurrence of a renewed consolidation process after retrieval of long-term memory, the question whether reconsolidation recapitulates consolidation is unresolved. We have previously reported in a double dissociation study (Bahar et al., Eur. J. Neurosci. 2003 $17 \cdot 1527$ ) that whereas inhibition of protein synthesis in the amygdalar central nucleus (CeA) disrupts consolidation of a newly acquired conditioned taste aversion (CTA) memory in the rat, a similar intervention in the basolateral nuclei (BLA) has no affect. Conversely, inhibition of protein synthesis in the BLA but not in the CeA, blocks experimental extinction of CTA memory. We have set out to investigate whether the amygdalar nuclei that are involved in the consolidation of the newly acquired memory (CeA) or in its experimental extinction (BLA), are also involved in reconsolidation. Toward this end, we microinfused into the CeA or the BLA the protein synthesis inhibitor anisomycin during retrieval of CTA memory. Retrieval was performed under conditions that lead to reconsolidation of the retrieved CTA memory. No effect was observed on the trace after retrieval, under the conditions that block consolidation (CeA) or experimental extinction (BLA) These results suggest that both amygdalar nuclei are not involved in the process of reconsolidation of CTA memory, and moreover, even if they do subserve reconsolidation in the intact brain, the molecular mechanisms involved in consolidation and reconsolidation, respectively, are different All in all, these data are incongruent with the hypothesis that reconsolidation recapitulates consolidation.

Supported by the Israeli Science Foundation, The Human frontiers Program, and the Volkswagen Foundation.

Mode of inhibition of phospholipase $A 2$ activity of B-bungarotoxin by a characteristic library-derived peptide Balass M., Kalef E. and Katchalski-Katzir E.

Dept. of Biological Chemistry, Weizmann Institute of Science. Rehovot 76100

$\beta$-bungarotoxin ( $\beta-\mathrm{BTX}$ ), one of the major neurotoxins in the venom of the snake Bungarus multicinctus, is targeted to the pre-synaptic membrane at the neuromuscular junction. $\beta$-BTX is a heterodimer protein that consists of two chains, chain $A$ (phospholipase $A_{2}$ subunit) and chain B (targeting subunit that interacts with certain types of potassium channels), that are linked together by a single disulfide bond. Employing a combinatorial phage peptide-library, we selected, by means of $\beta$-BTX, four different peptides having a consensus sequence WD(E)xLxxL. We show that a library-derived peptide 
(CAEVSTWEMLQQLNTTRMPPPC) binds to chain A of the toxin and inhibits its phospholipase $A_{2}\left(P L A_{2}\right)$ activity. This peptide inhibits PLA activity of $\beta$-BTX fraction as well as the $\mathrm{PLA}_{2}$ activity of the whole venom. Moreover, when this peptide was injected into mice concomitantly with $\beta$-BTX, it fully neutralized the toxin's lethality. Following HPLC analysis of the total isoforms of $\beta$-BTX on a hydrophobic column (C-8), we obtained five peaks (designated PI to P5) which represent the known five fractions of the majoi isoforms of the

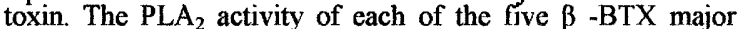
peaks is inhibited by the above peptide to a different degree We further purified a single $\beta-B T X$ isoform $\left(\beta_{1}-\mathrm{BTX}\right)$ and showed that the peptide inhibits non-competitively the purified isoform. As compared with some other snake toxins that have PLA $_{2}$ activity and a pancreatic PLA $A_{2}$ enzyme as well, we demonstrated that the above peptide inhibits to a different extent the enzymatic activity of the various $\mathrm{PLA}_{2}$ protein preparations.

Perceptual training generalizes to verbal working memory Banai K. and Ahissar M. ${ }^{2}$

Depts. of Neurobiology and Psychology ${ }^{2}$, Hebrew University Jerusalem

Psychoacoustic discrimination abilities and verbal working memory are significantly correlated, in a subpopulation of dyslexics with additional learning problems. Yet the direction of causality is not clear. Furthermore, the relations between mechanisms underlying verbal and non-verbal functions of working memory are not clear. In this study we first compared frequency discrimination thresholds of learning disabled $8^{\text {th }}$ grade students on two tasks of 2-tone frequency judgments. Students with poor high/low judgments also had poor verbal working memory scores, though they had no deficits in same/different judgments. We concluded that the deficit of these individuals is not at the level of signal representation but rather at the working memory level. To test this conclusion, we trained the group of learning disabled students with poorest frequency discrimination thresholds $(n=12)$ on the higher/lower task on other simple psychoacoustic tasks (e.g. longeristiorter in duration discrimination) for 8 weeks. Trained students improved greatly on all trained tasks. Improvement transferred uniquely to verbal working memory but not to other cognitive tasks or reading. This improvement lasted at least 5 months post training. We propose that the deficit of LDs in 2-tone frequency discrimination reflects a generalized working memory deficit. Practicing this task generalizes to improved working memory functions also with verbal material indicating that common mechanisms underlie the processing of verbal and non-verbal stimuli.

\section{Synaptic dynamics and efficacy estimated in a} biologically-realistic model of a spiny stellate cell of cat

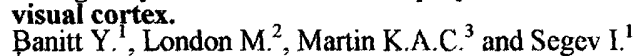

${ }^{1}$ Institute for Life Science, Hebrew University Jerusalem; ${ }^{2}$ University College, London; Institute of Neuroinformatics, Zurich.

Spiny stellate cells (SSC's) are the most common cell type in layer 4 (L4) of sensory cortex and are the major recipients of input from the sensory thalamus. Virtually all SSCs in cat visual cortex have simple receptive fields (Martin \& Whitteridge, J. Physiol. 353:463-504, 1984). Although traditionally, models of simple cells use only feedforward input from the lateral geniculate nucleus ( $L G N$ ) of the thalamus, not all excitation is from the LGN: SSC $:$ are excited by other L4 SSCs and by layer 6 pyramidal neurons (L6) (Stratford et al., Nature 1996). Inhibition comes mainly from small basket cells in layer 4. A biologically-realistic compartmental model for a SSC was built, which incorporated all four synaptic inputs. Based on morphological data, we distributed 360 LGN boutons, 1440 L4 boutons, 3108 L6 boutons and 357 inhibitory boutons over the dendritic surface of the SSC model. Modeled synapses were activated individually to match synaptic parameters (amplitudes, shape indices and dynamics) of the corresponding PSPs measured in-vitro. The model predicts a large synaptic conductance per LGN synapse. The information efficacy of LGN synapses decays significantly (up to 10 foli) as a function of their distance from the soma. The model predicts a long-lasting inhibitory conductance with a large peak. It also shows that, paradoxically, a facilitatory conductance underlies the apparently depressing IPSP amplitudes observed experimentally. The LGN contributes less than $10 \%$ of the excitatory synapses. The model shows that the contribution of the LGN synapses to the total excitatory current diminishes rapidly with time. If the entire LGN input produces an uncorrelated (random) barrage, only a few spikes are evoked in the SSC because the LGN synapses depress sharply. The inhibitory input, which is relatively more proximal, is powerful and capable of effectively controlling the gain of the recurrent cortical component of the total excitation.

The neuroprotective activity of rasagiline and its propargyl moiety is dependent on activation/gene expression of protein kinase C (PKC)

Bar-Am O. Amit T., Weinreb O., Yogev-Falach M. Chillag-Talmor $\mathrm{O}$. and Youdim $\mathrm{MBH}$.

Eve Topf and US National Parkinson's Foundation Centers of Excellence for Neurodegenerative diseases and Dept.s of Pharmacology, Technion, Haifa.

Rasagiline (N-propargyl-(1R)-aminoindan), a highly potent selective irreversible monoamine oxidase (MAO)- $\mathrm{B}$ inhibitor has been shown to suppress apoptosis induced by neurotoxins and oxidative stress not related to its MAO-B inhibitory activity. It has finished its final phase III clinical trials for Parkinson's disease (PD) and submitted to FDA for approval In the present study, we show that rasagiline (0.1-1 $\mu \mathrm{M})$ significantly reduced cell death induced by serum deprivation in rat $\mathrm{PC} 12$ cells and in SH-SY5Y neuroblastoma cells after 24 $h-48 \mathrm{~h}(-30 \%)$. Similar effects were also demonstrated for propargylamine, the propargyl moiety present in rasagiline Since PKC plays a key role in many cellular events, including regulation of apoptosis, we further investigated the effects of rasagiline on $\mathrm{PKC}$ activation and gene expression. Rasagiline dose-dependently $(0.1-10 \mu \mathrm{M})$ induced $\mathrm{PKC}$ phosphorylation (using p-PKC (pan) $\mathrm{Ab}$, detecting $\mathrm{PKC} \alpha, \beta_{\mathrm{I}}, \beta_{\mathrm{II}}, \zeta, \varepsilon, \eta$ and $\delta$ isoforms) as well as translocation of the isoforms PKC $\alpha$ and PKC $\varepsilon$ to the membrane. These effects were blocked by the PKC inhibitor, GF109203X. Moreover, propargylamine similarly and significantly increased p-PKC (pan) levels. Additionally, using real-time RT-PCR, we show that rasagiline elevated PKC $\alpha$ and PKC 8 gene expression after $24 \mathrm{~h}$ of serum deprivation

These results indicate that the neuroprotective activity of rasagiline, which is linked to $\mathrm{Bcl}-2$ family proteins at mitochondrial permeability transition pore, is mediated by modulation of PKC activation and gene expression. Furthermore by comparing the actions of rasagiline with propargylamine, we have been able to demonstrate that the propargyl moiety of rasagiline has the crucial role in these effects

PRS-211,375, a novel selective CB2 receptor agonist, demonstrates analgesic activity in several animal models Bar-Joseph A., Fink G., Richstein A., Dar D.E., Amselem S Bar-Ilan A., Avidor B., Yacovan A. Weksler A. and Meilin S Pharmos Ltd, Kiryat Weizmann, Rehovot 76326

Cannabinoid receptor agonists inhibit responses to painful stimuli by activation of the CBI receptor, located mainly in the CNS, and the CB2 receptor, expressed mainly by inflammatory and immune cells. PRS-211,375, a selective $\mathrm{CB} 2$ receptor agonist (CB2 affinity $\mathrm{IC}_{50} \sim 1 \mathrm{nM}$ ) was tested for its analgesic effect using 3 experimental animal pain models. The models were: (i) the tail-flick in mice for noxious pain; (ii) carrageenan-induced thermal and mechanical hyperalgesia in the rat hind paw for inflammatory pain; (iii) IP acetic acid induced visceral pain in the mice. PRS-211,375 showed relatively little analgesia in the tail flick test. However, PRS-211 375 administered either IP or PO showed significant dose-dependent analgesia (a decrease of more then $50 \%$; $\mathrm{p}<0.05$ ) in the carrageenan-induced inflammatory paw test (both in the thermal and mechanical stimuli). The compound also reduced paw edema volume in a dose-dependent manner $(30 \mathrm{mo} / \mathrm{kg} \mathrm{PO}$ reduced the edema by $50 \%$ ). Pre-treatment with PRS-211,375 significantly reduced visceral pain as assessed by a reduction in writhing counted during 5 minutes $(0.1 \pm 0.1$ compared with $25.7 \pm 1.3$ in vehicle-treated control animals; $p<0.01$ ). PRS-211,375 proved to be much more potent than NSAIDS tested in the visceral pain model (Ketoprofen, Diclofenac and Celecoxib). Administration of a CB2, but not CB1 antagonist significantly reversed the effect of PRS-211,375 in both the inflammatory and visceral pain models suggesting that the analgesic activity of PRS-211,375 is mediated by the CB2 receptor.

These data indicate that PRS-211,375, a CB2 selective agonist may be a potent analgesic compound for treatment of inflammatory related pain. 
Neuronal recruitment in adult zebra finch brain during a reproductive cycle

Barkan S. ${ }^{1}$, Ayali A. ${ }^{1}$, Nottebohm F. ${ }^{2}$ and Barnea A. ${ }^{3}$

${ }^{7}$ Dept. Of Zoology, Tel-Aviv University, Israel; ${ }^{2}$ The

Rockefeller University, Field research center, NY USA ${ }^{3}$

Dept. of Natural Sciences, The Open University of Israel

The occurrence of neurogenesis (birth of new neurons) and new neuronal recruitment

in adult brains is by now a well known phenomenon, found in several warm-blooded vertebrates, including humans. The hypothesis is that this phenomenon serves adaptive functions and contributes to the cellular basis of brain plasticity and ability to acquire new long-term memories. Accordingly, regulation of new neuron survival by extent of circuit use may be a general mechanism for ensuring that neuronal replacement is closely attuned to environmental change and to the need to store novel information.

Previous studies in our laboratory support this hypothesis and show that in adult birds, increase in information load is associated with an increased recruitment of new neurons: when adult birds are exposed to a new complex social setting, more neurons are recruited in parts of their brains that process auditory and visual information, than in brains of other birds which were exposed to a simple setting. These findings indicate that social complexity might affect neuronal survival. Along this line, the present work is designed to further test the hypothesis that times when new and heavy memory loads have to be handled coincide with the recruitment of new neurons. To do this, we use an estrildid songbird - zebra finch (Taeniopygia guttata) - as a model system. This species is highly social and in nature it nests colonially and forages in large flocks of up to several hundred individuals. Nestlings remain in the nest for about two weeks and during this period they depend exclusively on their parents. After fledging when the young birds leave the nest and join with other juveniles in the colony, their parents keep feeding them for two more weeks until they reach independence. This suggests that prior to this stage parents must learn to recognize their offspring. Recognition can be elicited via visual as well as auditory or even olfactory cues. The hypothesis to be tested is that changes in hormonal profile during egg laying, incubation or rearing of the young, and attendant changes in experience, may trigger a sharp increase of new neurons into relevant parts of the brain, to enable parents-offspring recognition when the young have to be fed until they reach independence.

To do this, we inject adult breeding birds (males and females) with [3]H-thymidine, a cell birth marker, during different stages of their reproductive cycle, as follows:

1. control: birds are treated and then kept singly; these birds do not engage in reproduction. 2. birds are treated and two weeks later are paired and allowed to breed. 3. birds are paired allowed to breed and treated after the last egg is laid. 4. bird are paired, allowed to breed and treated when their juveniles fledge from the nest.

Forty days later we record the number of new neurons in two brain regions: neostriatum caudale (NC. a forebrain region that processes auditory and visual information) and the olfactory bulb. A comparison between experimental groups will be presented and discussed

Explicit and implicit aspects of Kanizsa figure detection and discrimination

Barlasov A. and Hochstein S.

Neurobiology Dept., Institute of Life Sciences \& Neural Computation Center, University of Jerusalem, 91904 Jerusalem

Previous studies suggested that perception of an illusory contour, such as a Kanizsa-type figure, might involve a temporal sequence of stages (Reynolds, 1981 Perception 10: 107; Ringach \& Shapley, 1996 Vision Res 36: 3037). We were interested in the cortical level(s) of these stages in reference to Reverse Hierarchy Theory (RHT; Hochstein \& Ahissar, 2002 Neuron 36: 791). We presented subjects with four inducing figures ("pacmen"), which were arranged as if at the corners of a 60-degree parallelogram. The openings of the pacmen were aligned so as to induce an illusory Kanizsa parallelogram $(25 \%$ of trials), a Kanizsa equilateral triangle $(25 \%)$, or neither $(50 \%)$. Non-inducing pacman openings were unaligned or faced outward. Presentations were brief and backward-masked (SOA of 50-200ms). Subjects reported whether they perceived a Kanizsa figure (detection) and which shape they saw (discrimination) - "guessing" the shape even when it was not explicitly perceived. Performance improved with SOA, as expected. Surprisingly, for longer SOAs, even when subjects did not explicitly perceive the figure, they were significantly above chance at guessing which shape had been presented (d'>1), suggesting implicit figure perception. An alternative, that figure discrimination without awareness depends on detection of local features was ruled out by the following control: For triangle-shaped figures, when the fourth inducer faced outward, this local cue was used as a signal of a triangle shape only when the figure was perceived explicitly and for longer SOAs. These findings support the RHT view that associates focused attention to local cues with late explicit processing at low-level cortical areas.

Support: Israel Science Foundation (ISF) "Center of Excellence" Grant; US-Israel Binational Science Foundation (BSF)

\section{Social change affects the turnover of new neurons in the} forebrain of adult songbirds

Barnea A. ${ }^{1}$, Mishal A. ${ }^{2}$, Rado $\mathrm{R}^{2}$ and Nottebohm $\mathrm{F}^{3}$ Natural and Life Sciences, The Open University of Israel. ${ }^{2}$ Zoology, Tel-Aviv University,Israel; ${ }^{3}$ The Rockefeller University, NY, USA

Many new neurons are added to the adult avian brain. Most of them die $3-5$ weeks after they are born. Those that survive replace, numerically, older ones that have died. It has been suggested that the new neurons enhance the brain's ability to acquire new long-term memories. Previous work in our laboratory support this hypothesis and show that in aduit birds increase in information load is associated with an increased recruitment of new neurons: when adult birds (Taeniopygia guttata) are exposed to a new complex social setting and tested 40 days later, more neurons are recruited in parts of their brain that process auditory information, than in brains of other birds which were exposed to a simple setting and tested at the same time. This finding indicates that social complexity might affect neuronal survival. Along this line, the present work was designed to test whether social complexity also affects the longevity of these new neurons. To do this, we treated adults birds with ${ }^{[3]} \mathrm{H}$-thymidine immediately before introducing them into one of two new social settings: complex (40 strange birds) or simple (isolation). We tested their brains, in addition to 40 days later, also at 60 and 150 days after treatment. The results show that the higher level of recruitment found in the complex social setting after 40 days is followed by a higher rate of neurons disappearance than that seen in the simple one. In this light social setting seems like a dynamic situation that calls for many new neurons which in turn, tend to be replaced promptly, so that overall there is a greater turnover of neurons in the more complex social setting. These results further support the hypothesis that regulation of new neuron survival by extent of circuit use may be a general mechanism for ensuring that neuronal replacement is closely attuned to environmental change and to the need to store novel information.

\section{Sex differences in response to neurofeedback treatment}

Barnea A ${ }^{1}$, Rassis A ${ }^{1}$ and Zaidel E.

${ }^{1}$ Bio-Keshev Clinic, Kibutz Givat Chaim Ichud, Israel, ${ }^{2}$ Dept. of Psychology, UCIA, USA

Methods: Human subjects can learn to control their brain waves parameters (Neurofeedback or NF) when given reinforcement via instantaneous feedback. The parameters we used for giving feedback were spectral powers in different frequency bands. We applied a Neurofeedback training protocol that rewarded reducing the power in the theta (4-7 $\mathrm{Hz}$ ) band and enhancing the power in the SMR $(12-15 \mathrm{~Hz})$ band at central sites of 20 Israeli children aged 10-12, half boys and half girls. Half of the subjects received C3 training and the other half $\mathrm{C} 4$ training. Training consisted of 20 half-hour sessions. We assessed the effects of training on lateralized lexical decision in Hebrew, on dichotic listening to nonsense stop consonant-vowel syllables, as well as on computerized cognitive tasks, including the IVA CPT, Spatial Orientation, Nonverbal IQ, Picture Memory and Word Memory. Results: There were surprising sex differences in the effects of training. In lateralized lexical decision, both $\mathrm{C} 3$ and C4 feedback affected both hemispheres, though differently in boys and girls. Thus, in boys $\mathrm{C} 3$ training impoved right hemisphere accuracy of word recognition, and $\mathrm{C} 4$ training improved left hemisphere accuracy, whereas in girls both $\mathrm{C} 3$ and $\mathrm{C} 4$ training improved left hemisphere accuracy. In the dichotic listening test, NF increased the right ear advantage (REA) in girls, signaling increased left hemisphere specialization for phonetic perception, but NF decreased the REA in boys, thus shifting the greater asymmetry from boys to girls. Here, NF on either side affected hemispheric specialization (in girls) as well as callosal transfer (in boys). $\mathrm{C} 4$ feedback improved the IVA Auditory Speed in girls whereas $\mathrm{C} 3$ feedback improved it in bovs. Conclusion: Laterality of NF can affect asymmetric control networks (as in lexical decision), while feedback on either side can affect the same lateralized processing modules or the same callosal channels (as in dichotic listening). We suggest that the NF protocol activates hemispheric control circuits that are organized differently in boys and girls. 
Intrinsic protein synthesis regulation by eukaryotic elongation factor $\boldsymbol{z}$ (eEF2) is correlated with memory consolidation

Belelovsky K. ${ }^{1}$, Elkobi A. ${ }^{1}$, Nairn A.C. ${ }^{2}$ and Rosenblum K. ${ }^{1}$ 'Center for Brain and Behavior, Haifa University, Haifa

Israel; ${ }^{2}$ Yale University Sch. Med., New Haven, CT, USA.

One of the most intriguing questions in neurobiology is to understand the neural mechanisms of long-term memory formation. Studies have shown that the initial stage of memory formation, a consolidation process, is dependent on protein synthesis in a number of species, following different learning paradigms. The necessity of intact protein synthesis machinery for memory consolidation suggests that regulation of protein synthesis in neurons is a crucial component of the consolidation process. However, it is not clear whether neurons regulate protein translation during memory consolidation, and if so, what proteins are involved in this process. This study was conducted in order to learn whether active regulation of protein synthesis plays a role in memory consolidation. We report here that phosphorylation of eukaryotic elongation factor-2 (eEF2) on Thr56 by eEF2 kinase in the insular cortex is correlated with taste memory consolidation. We found a significant increase (22\%) in eEF2 phosphorylation $20 \mathrm{~min}$ following learning, but not at other time points. The temporal pattern of eEF2 phosphorylation is similar to ERKI/II activation. The phosphorylation of both proteins is specific to the taste cortex. In addition, eEF2 phosphorylation during memory consolidation is detected in a synaptosomal fraction made from taste cortex. The results provide for the first time possible evidence for the involvement of active synaptosomal translation regulation during the formation of long-term memory trace.

Di-isopropylfluorophosphate (DFP) subtoxic exposure at early postnatal critical period impairs performance and acquisition of passive avoidance in female, but not male adult mice

Ben Bashat $\mathrm{G}$ and Kofman $\mathrm{O}$.

Dept. of Behavioral Sciences and Zlotowski Center for

Neurosciences, Ben-Gurion University, Beer Sheva

Background: ACh plays a major role in encoding, attention and regulation of cortical development, and sex differences were found for behavioral effects of AChE-I's. Acetylcholinesterase inhibitors (AChEI) are widely used as pesticides, exposing a substantial population to the risk of neurological damage. A single exposure to AChEIs in 3-10 day old mice caused down-regulation of muscarinic receptors, impaired spatial learning and altered motor behavior in females only (Dam et al. 2000). The present study examined the effect of chronic developmental exposure to AChEI on passive avoidance (PA) learning.

Methods: C57BL/65 mice of both sexes were injected with $1 \mathrm{mg} / \mathrm{kg}$ sc DFP or saline on postnatal days 4-10 or PND 14-20 in a second experiment or, in a third experiment, on PND 30-36. Step-down PA was conditioned at 4 months using 0.5 $\mathrm{mA}$ footshock for $5 \mathrm{sec}$ and tested after $24 \mathrm{hr}$ and $72 \mathrm{hr}$ Resuits: After treatment at PND 4-10, DFP females, but not males showed no impairment in acquisition, but a significant impairment in retention at $24 \mathrm{hr}(\mathrm{F} 2,104=4.30, \mathrm{p}<0.02)$. Treatment on days 14-20 impaired acquisition in females and a trend towards an impairment in retention at $24 \mathrm{hr}$, but not at 72 $\mathrm{hr}$, suggesting latent learning of PA. No difference between male DFP and control mice was seen after $24 \mathrm{hr}$, but $72 \mathrm{hr}$ after acquisition, step down latencies in control, but not DFP males declined significantly. DFP on PND 30-36 did not impair acquisition or retention in either sex.

Conclusions: The results suggest that in females DFP affects the rate of acquisition or retention differentially when administered at different time points during development.

The $M_{2}$ and $M_{1}$ muscarinic $G$-protein-coupled receptors are voltage sensitive

Ben-Chaim Y. ${ }_{1}^{1}$ Tour O., ${ }^{1,2}$ Dascal N., ${ }^{3}$ Parnas I., ${ }^{1}$ and Parnas H. The Otto Loewi Minerva Center for Cellular and Molecular Neurobiology, the Hebrew University, Jerusalem 91904

Israel; 'Presently at the Dept. of Pharmacology, University of California San Diego, La Jolla. CA 92093-0647, USA; Dept. of Physiology and Pharmacology. Tel Aviv University, TelAviv 69978. Israel.

G-protein coupled receptors (GPCRs), involved in the majority of signal transduction processes in the body, act mainly by activation of second messengers within the cell. The regulation of the GPCRs activity is not considered to be affected by membrane potential. Here, using Xenopus oocytes, we show that the $M_{2}$ and the $M_{1}$ muscarinic receptor ( $m 2 R$ and $m I R$ ) are voltage sensitive. The $\mathrm{m} 2 \mathrm{R}$-mediated potassium channel (GIRK) currents were used to assay the activity of the $m 2 R$ We found that the apparent affinity of the $m 2 R$ toward acetylcholine ( $\mathrm{ACh}$ ) was reduced upon depolarization. Binding experiments of $\left[{ }^{3} \mathrm{H}\right]$. ACh to individual oocyte expressing $\mathrm{m} 2 \mathrm{R}$ confirmed the electrophysiological findings. We further found that the voltage sensitivity of binding of ${ }^{3} \mathrm{H} \mid-\mathrm{ACh}$ to oocytes expressing $\mathrm{m} 2 \mathrm{R}$ was greatly diminished following pretreatment with pertussis toxin. In contrast to the $m 2 R$, the apparent affinity of the $\mathrm{mlR}$ was increased upon depolarization. However, when the third intracellular loop of the $m 1 R$ was replaced by the same loop of the $m 2 R$, the receptor lost its voltage sensitivity. The cumulative results suggest that the $\mathrm{mlR}$ and $\mathrm{m} 2 \mathrm{R}$ are, by themselves, voltage sensitive. Furthermore, the voltage sensitivity does not reside in the ACh binding site, rather it most likely resides in the receptor region that couples to the G-protein

These finding may be of significant physiological importance as they imply that membrane potential may affect the regulatory activity of GPCRs.

\section{Adult human bone marrow stromal cells express} transcription factors following in vitro induction Ben-ZurT. ${ }_{\text {Melamed E. }}$. Lulvik S. B. , and Offen D.

${ }^{I}$ Felsenstein Medical Research Center, Dept. of Neurology, Felsenstein Medical Research Center, Dept. of Neurology,
Rabin Medical Center. Sackler School of Medicine, Tel Aviv University; 'Laniado Medical Center, Natanya

Human mesenchymal stem cells present in adult marrow can develop into bone, cartilage, fat, tendon and muscle. In this study, we demonstrate that human bone marrow stromal cells (hBMSc) isolated from human iliac crest can be induced to differentiate into dopaminergic neural-like cells. The hBMSc were analyzed by flow-cytometry and were found negative for lymphohematopoietic cell surface markers such as CD5, $\mathrm{CD} 1 \mathrm{lb}, \mathrm{CD} 20, \mathrm{CD} 34$ and CD45. However, they express CD90 a protein that plays a role during synaptogenesis. A few hours after $\mathrm{hBMSc}$ were incubated with $\mathrm{N} 2$ supplements, retinoic acid, butylated hydroxyanisole, isobutylmethylxanthine and dibutyryl-cyclic-AMP, the cells changed their phenotype to neural-like cells, spindle shaped, with cell bodies and long branches. Using protein/DNA array (Panomics Inc.) we purified and incubated the nuclear proteins with labeled DNA and probed the bound DNA to transcription factors consensus sequences microarray. In the differentiated hBMSc, using this array technology, we showed induced expression of several neuron-specific transcription factors such as glycosaminoglycan (GAG) and the neural zinc finger factor $(N Z F-3)$. The characterization of specific transcription factors is the key for successful induction of hBMSc to become neural-like cells.

\section{Functional integration of transplanted embryonic stem} cell-derived neurons

Benninger F ${ }^{1,2}$, Beck $\mathrm{H}^{2}$, Wernig M.', Tucker K.L. ${ }^{4}$, Bröstle $\mathrm{O}^{\top}$ and Scheffler $\mathrm{B}$.

Institute of Reconstructive Neurobiology and Dept.s of ${ }^{2}$ Epileptology and ${ }^{3}$ Neuropathology, University of Bonn Medical Center. D-53105 Bonn, Germany, and

${ }^{4}$ Interdisciplinary Center for Neurosciences, University of Heidelberg, D-69120 Heidelberg, Germany

The generation of neurons and glia from pluripotent embryonic stem (ES) cells represents a promising strategy for the study of central nervous system (CNS) development and repair. ES cell-derived neural precursors have been shown to develop into morphologically mature neurons and glia when grafted into brain and spinal cord. Yet, there is a surprising shortage of data concerning the functional integration of ES cell-derived neurons (ESNs) into the host CNS tissue. Here, we use ES cells engineered to express enhanced green fluorescent protein (EGFP) only in neuronal progeny to study the functional properties of ESNs upon integration into long-term hippocampal slice cultures. Following incorporation into the dentate gyrus, EGFP $^{+}$donor neurons display a gradual maturation of their intrinsic discharge behavior and a concomitant increase in the density of voltage-gated $\mathrm{Na}^{+}$and $\mathrm{K}^{+}$channels. Integrated ESNs express AMPA and GABA receptor subunits. Most importantly, neurons derived from ES cells receive functional glutamatergic and GABAergic synapses from host neurons.

Specifically, we demonstrate that host perforant path axons form synapses onto integrated ESNs. These synapses between host and ES cell-derived neurons display pronounced paired-pulse facilitation indicative of intact presynaptic short-term plasticity. Thus, ES cell-derived neural precursors generate functionally active neurons capable of integrating into the brain circuitry. 
Enriched environment in mice: Elevates natural killer cell activity and attenuates psychological stress response Benaroya-Milshtein N. ${ }^{1}$, Hollander $\mathrm{N}^{2}$, Apter A. ${ }^{3}$, Wilf A. ${ }^{1}$, Yaniv 1 . and Pick C.G.

Deps ${ }^{1}$ Anatomy, ${ }^{2}$ Human Microbiology, Tel Aviv University, Deps ${ }^{3}$ Psychiatry ${ }^{4}$ Hemato-Oncology, Schneider Children's Medical Center of Israel

The importance of the environment in the regulation of brain, behavior and physiology has long been recognized in the biological, social and medical sciences. Animals from enriched conditions clearly have better learning abilities than animals from standard conditions. However, the effects of environmental enrichment on immunology and emotionality are less documented and remain questionable. Moreover, mice are less investigated regarding enriched environment. Therefore, we investigated the influence of enriched environment on natural killer cell activity, psychological stress response and behavioral parameters. Male $\mathrm{C} 3 \mathrm{H}$ mice were raised either in enriched or standard conditions for 6 weeks. Afterwards, NK cell activity was measured and mice behavior was tested in the staircase test and Elevated plus maze. In addition, electric shock with reminders was given, in each reminder freezing time was measured. The $\mathrm{C} 3 \mathrm{H}$ mice in the enriched condition had higher cytolytic activity than did the standard group $(\mathrm{p}<0.001)$. The $\mathrm{C} 3 \mathrm{H}$ mice in the enriched condition showed lower freezing time in the reminders $(p<0.05)$, decreased anxiety and higher activity compared to standard. For instance, greater percentage of time spending in the open arms $(p<0.01)$ in the Elevated plus test, and higher rate of stair climbing in the staircase test $(p<0.05)$. The beneficial effect of enriched environment on NK cell activity in $\mathrm{C} 3 \mathrm{H}$ mice is clear. Enriched condition reduces anxiety as seen in the elevated plus test and in lower freezing time after psychological stress. It is still unclear which component of the enriched environment is responsible for these changes.

Sniffing with the mind's nose: Olfactomotor activity during imagery mimics that during perception

Bensafi M., Khan R. and Sobel N.

Helen Wills Neuroscience Institute, UC Berkeley, Berkeley, CA 94720, USA

Neural representations created in the absence of appropriate external events are referred to as mental imagery. Imagery may be augmented by reenactment or "playback" of sensory-motor processes. For example in vision, occulomotor responses during imagery are correlated with those during perception. Furthermore, this occulomotor activity functionally contributes to the quality of visual mental imagery. like visual perception, odor perception requires a sensory (smelling) and a motor (sniffing) integration that is mediated by dissociable neural networks. Here we set out to ask whether the olfactomotor response (sniffing) is enacted during olfactory mental imagery. We measured nasal airflow in 30 human subjects who were imagining sights, sounds, and smells. Subjects were unaware of airflow measurement. Only during olfactory imagery, humans spontaneously enacted the sensory-motor componen of olfaction, that is, they sniffed $\left(F_{3,87}=22.290, P<0.0001\right)$. Moreover, as in perception, imagery of pleasant odors involved larger sniffs than imagery of unpleasant odors $\left(F_{1,449}=4.854 P<0.029\right)$. Finally, encouraging or preventing sniffing, increased or decreased the vividness of olfactory mental imagery respectively $\left(F_{1,19}=6.192, P<0.023\right)$. These findings suggest that sniffs play a functional role in the creation of olfactory percepts.

\section{Development of a bioassay of exposure to environmental} hazardous agents

Berman-Shlomovich T. and Wormser U.

Hebrew University of Jerusalem, Faculty of Natural Sciences One of the major limitations of current methods of biological detection of exposure to hazardous environmental agents is their inability to detect long-term exposures, and exposures that occurred in the past without immediate clinical effects. In the current study we examined the potential of a new bioassay that is based on the hypothesis that scrum of exposed individuals contains a toxic factor or factors produced by an affected cell or tissue in response to an exposure. In the present study PC12 cell cultures were exposed to serum samples of rats treated with the organophosphate chlorpyrifos. We observed a decrease of up to $31.8 \%$ and $40 \%$ in cell viability and nerve growth factor-induced neurite outgrowth respectively, in PCI2 cells treated with serum of chlorpyrifos-exposed rats, in comparison to cells treated with serum of control rats. Maximal effect was observed for both parameters 4 weeks after exposure whereas motor activity and cholinesterase activity returned to normal levels within one week after the exposure; thereafter the rats showed no signs of toxicity, including 4 weeks post-exposure. These results demonstrate the potential of the proposed method to detect environmental exposures long after they have occurred.
Behavioral analysis of multiple memory tasks in monkeys Bernacchia A ${ }^{2}$, Yakovlev V. ${ }^{\text {, }}$, Hochstein S. , Orlov T. ${ }^{1}$ and Amit DJ ${ }^{1}$

'Hebrew University, Jerusalem 91904 Israel; ${ }^{2}$ Universita' di Roma, La Sapienza, 00185 Italy:

We investigated primate performance in a behavioral task that requires holding several visual images simultaneously in working memory. Two Macaque monkeys were trained on a delayed-match-to-sample task with multiple samples. In each trial, a sequence of 1-7 different images served as samples. These were followed by a match stimulus, which was a repetition of one of the samples, (the cue). The length of the sequence and the position of the cue in the sequence were chosen at random on each trial. The monkey's task was to recognize the appearance of a repetition of any one of the samples and therefore it needed to hold in working memory all the images of the sequence. Two type of errors occurred:

"Misses": a failure to detect the repetition. We found that the distribution of these errors depended on the position of the cue in the sample sequence: The greater the cue-match separation, the poorer the performance. Surprisingly, for fixed cue-to-match separation, performance improved with increasing sequence length, i.e. with the number of items in working memory,

"False positives": a response erronously reporting repetition, folowing a sample stimulus presented for the first time in the current trial. Distribution of these errors increased with the time and number of stimuli since the beginning of the trial. Our findings are consistent with a model (Amit et al., Cerebral Cortex, 2003) suggestng that noise, associated with neural dynamics, spontaneously erases items from working memory (leading to misses) and induces "ghost" memories of familiar, unseen stimuli (leading to false positive errors)

Supported by the Israel Science Foundation (ISF) Grant $\# 8009$.

\section{Modeling the BOLD MRI flow signal}

Bick A. ${ }^{1,2}$ and Goelman $\mathrm{G}$

${ }^{7}$ MRIMRS lab, HBRC, Medical biophysics and nuclear medicine dept., Hadassah - Hebrew University Hospital;

${ }^{2} I C N C$, Hebrew University

In order to achieve a better understanding of the processes underlying blood oxygenation level-dependent (BOLD) MRI we conducted a simulation. Its aim was to achieve a reliable tool for testing and generating hypotheses about brain connectivity seen with MRI. We utilized the Monte Carlo simulation first presented in Ogawa et al (Biophys, 64, 803 ,1993) and developed further in Boxerman et al (MRM 34, 555 ,1995). This model calculates the expected signal attenuation due to phase dispersion in a voxel that contains an assembly of vessels. It uses a random walker proton to simulate diffusion. The signal is the average of the collected phase $<\mathrm{e}^{\mathrm{I}},<^{3}$ when the averaging is done over the organization of the vessels in the voxel and the possible paths of the random walker in the medium. The averaging is done using $\mathrm{MC}$ methods by averaging the signal of $\mathrm{N}$ random protons.

This method was developed to calculate the signal at a given time in a single voxel, however it is too slow to allow the simulation of many voxels in different times needed for simulating flow. We therefore chose to apply an analytic method that calculates statistically the approximate value of the desired averages. The Analytical method (Kiselev \& Posse MRM 41, 499,1999 ) is based on solving the Torrey-Bloch equation (Torrey, Phys Rev 104, 563, 1956) and calculating the mean assuming random distributions. The means were calculated numerically resulting in full analytic forms of the signal. There is good agreement between the results from both methods, and therefore the analytical method can substitute for the MC method. Simulating connectivity requires adding flow to the model. The parameters in the model that can vary as a result of flow are the deoxyhemoglobin level, the CBV, and distribution of radii of vessels in the voxel. The simulation necessitates understanding the regularities that underlie the dynamics between voxels and the structure of the vascular system. These are our current goals.

\section{Regional modulation of NMDA receptor density by age} and brain injury

Biegon A. Fry P. and Paden C.

Dept. Imaging, LBNL, CA USA and Dept. Cell biology and Neuroscience; Montana State Univ. Bozeman. MT USA

Non-fatal brain injury is often associated with long lasting cognitive deficits and is recognized as a highly significant ris factor for Alzheimer's disease. Glutamate acting through NMDA receptors (NMDAR) is thought to be important in memory formation and is also implicated in the pathophysiology of traumatic and ischemic brain injury.

We have investigated age related changes in NMDAR density in normal control rats as well as in rats subjected to a 
penetrating (knife cut) brain injury. Regional receptor density was measured by quantitative autoradiography; using radioactive MK801 as a ligand. Male rats were subjected to the surgery at 7 weeks of age and groups of 3-6 killed 3 days, one month or 2 months later; in parallel with normal age-matched controls. Normal animals exhibited modest but statistically significant age-dependent loss of NMDA receptors in most brain regions studied. Injury produced large and statistically significant decreases in NMDAR density after 3 days; which were mostly but not exclusively limited to the ipsilateral hemisphere and to regions surrounding the knife track. Significant partial or even full recovery was observed 30 days after the lesion, such that NMDAR density in the injured animals was not significantly different from age matched controls. However, this was followed by a dramatic, bilateral reduction in NMDAR relative to age matched controls; suggesting that early brain injury sensitizes the brain to later age-dependent receptor loss. These changes, if relevant to humans, may contribute to the long-term cognitive deficits following brain injury as well as to the effect of head injury on the risk of Alzheimer's disease.

Endogenous 5-HT released from thalamic axons induces local disinhibition in developing layer 4 of mouse barrel cortex

Binshtok A.M., Fleidervish I.A. and Gutnick M.J.

Koret School of Veterinary Medicine, The Hebrew University of Jerusalem, Rehovot 76100

During the first two postnatal weeks, as thalamocortical (TC) axons grow into somatosensory cortex, arborize, form synapses in Layer 4 and orchestrate segregation of neurons into discrete "barrels", TC cells transiently express the serotonin transporters responsible for 5-HT uptake from the extracellular space and concentration in synaptic vesicles. Since these cells are not serotonergic, it has been suggested that they use 5-HT as a "borrowed" neurotransmitter. We previously demonstrated that in Layer 4 of mice younger than $\mathrm{P} 14$, exogenous 5-HT completely and reversibly blocks stimulus-elicited IPSCs and induces all-or-none epileptiform discharges that are similar to those induced by GABAA blockers. We also showed that these effects are mediated by presynaptic 5-HT2A receptors. Here, using thalamocortical slices from P6-P13 mice, in which the ventrobasal (VB) nucleus of thalamus, the TC axons and the "barrel" cortex are all present, we show that endogenous 5-HT released from TC axons mimics the effect of exogenous 5-HT Repetitive, low-intensity VB stimulation evoked long-lasting negative shifts in field potential (fp) recorded in the corresponding "barrel". Whole cell recordings from single Layer 4 spiny stellate cells revealed that these negative fps were accompanied at the cellular level by bursts of action potentials that appeared with varying latencies $(200-800 \mathrm{~ms})$ following VB stimulation. Both the negative fps and the underlying neuronal activities were completely and reversibly abolished by bath application of ketanserine tatrate $(20 \mu \mathrm{M})$, a specific 5-HT2A receptor antagonist. VB stimulation in slices from animals older than P14 did not elicit negative fps.

Our data support the hypothesis that during the time window when 5-HT becomes concentrated in presynaptic TC terminals thalamic discharge causes co-release of 5-HT along with glutamate, and thereby produces local disinhibition and consequent population bursts of synchronous cortical discharge. Such a feed-forward excitation mechanism may play a critical role in specific TC circuit maturation

Supported by grant 449/00 from the Israel Science Foundation

\section{$T_{1-}, T_{2^{-}}$and diffusion MRI of pig spinal cord in}

experimental allergic encephalomyelitis

Biton I.E. ', Mayk A.
'School of Chemistry, Tel Aviv University, Tel Aviv; ${ }^{2}$ Teva

Pharmaceutical Industries Ltd. Netanya; ${ }^{3}$ Functional Brain

Imaging Unit, Tel Aviv Sourasky Medical Center

Experimental allergic encephalomyelitis (EAE) is an immune-mediated inflammatory demyelinating animal model affecting the central nervous system. EAE pathology has similarities to multiple sclerosis (MS) in humans that takes a relapsing-remitting or a progressive course.

Tissue samples of control $(\mathrm{N}=3)$ and $E A E$ diseased $(\mathrm{N}=10)$ pig spinal cords (cervical) were used in this study. The EAE mode was induced in the pigs by immunization with bovine myelin basic protein, complete Freund's adjuvant and $M$. tuberculosis. The in vitro magnetic resonance imaging (MRI) protocol included saggital and transverse $T$-weighted images ( $T R / T E=700 / 20 \mathrm{~ms}$ ), $T_{2}$-weighted images (TR/TE $=3500 / 50 \mathrm{~ms}$ ) and diffusion-weighted images (DWI) (TR/TE $=1000 / 46 \mathrm{~ms}$, $\mathrm{D} / \mathrm{d}=200 / 10 \mathrm{~ms}, \mathrm{G}_{\max }=12 \mathrm{G} / \mathrm{cm} \mathrm{b}_{\max }=2 \times 10^{6} \mathrm{~s} / \mathrm{cm}^{2}, \mathrm{q}_{\max }=511$ $\mathrm{cm}^{-1}$ ), in which the diffusion was measured perpendicular and parallel to the long axis of the spine. Image analysis of the DWI data set was performed using a Matlab program to produce q-space displacement maps.

In seven from the ten EAE diseased spinal cords we found well defined plaques in the white matter, which were characterized by areas of increased intensity in the $T_{1}$-weighted images. $\mathrm{T}_{2}$-weighted images were also capable of identifying the lesions and demonstrated lesion heterogeneity in many cases. In nine from the ten EAE diseased spinal cords the q-space displacement maps, obtained from DWI, were different from that of the control maps both in the plaques $\left(p<2 \times 10^{-1}\right)$ and in the "normal appearing white matter" $\left(\mathrm{p}<6 \times 10^{-4}\right)$ of the EAE diseased spinal cords. The tenth case that did not showed any MRI abnormalities had less significantly clinical signs as compared to the other nine EAE diseased pigs.

This study provides the first identification of EAE abnormalities in pig spinal cords using MRI. The q-space displacement maps showed abnormalities in the white and gray matter that $T_{1}$ and $T_{2}$-weighted images could not identify.

"To boom or not to boom" - ' 7 BOOM' as a novel test in the diagnosis of ADHD

Biran I. and Gal A.

Dept. of Neurology, Hadasssah University Medical Center, Jerusalem

AIM: To establish the validity of the ' 7 Boom' task in the diagnosis of Attention Deficit Hyperactivity Disorder (ADHD). Introduction: Subjects with ADHD show a pattern of both inattention and impulsivity/hyperactivity. The diagnosis is based on the history, neurological examination and neuropsychological testing which demonstrate difficulties with tasks that require sustained attention and response inhibition. We adapted the game ' 7 Boom' which depends heavily on attention and compared the performance of ADHD patients in this task to that of normal controls. Methods: Subjects - 11 normal controls (age - 27.5 years \pm 10 , education - 14.5 years \pm 3.0 ); 8 ADHD patients - (age - 26.1 years \pm 9 , education 13.6 years \pm 3.6 ). The diagnosis was based on the DSM criteria and the performance in the Test of Variables of Attention (TOVA). Task - Based on the children's game ' 7 Boom', subjects counted alternating with the examiner from 1 to 100 . Whenever a number with the figure ' 7 ' (e.g. 27) or a multiple of ' 7 ' (e.g. 49) was reached the subjects were asked to say 'boom' instead of the number. The task was administered twice - in the first the examiner counted the odd numbers and the subject the even numbers and vice versa in the second. Results: ADHD subjects performed worse than the control subjects (14.6 errors $\pm 4.9,6.5$ errors \pm 2.9 respectively, $p=0.004$ ) Conclusion. The '7 Boom' task reflects the behavioral pattern of subjects with ADHD and can help in the diagnosis of this syndrome.

Convergence and "small world network" organization of dendrites in cultured networks: Effect on synaptic

distribution and clustering

Blinder $P$ and Baranes D

Dept. of Life Sciences, The Institute for Applied Research, Ben Gurion University of the Negev.

Dendrites of hippocampal neurons in culture form complex network morphologies We implemented graph theory to study such networks; we defined contacts between dendrites as vertices and dendritic segments between two vertices as edges. The mean distance from each vertex to all others vertices (path length $(L)$ ), and the level of contact among vertices neighbors (connectivity (C)) showed that the dendritic network architecture belongs the Small World Network (SWN) type SWNs combine elements of random and regular networks in a way that strongly lowers $L$ and increases $C$, yielding a highly connected network with small degree of separation among dendrodendritic contact sites. This is utilized by axons when they fasciculate on dendrites and turn to switch targets at the vertices, minimizing the growth length required to connect distant cells. Around 15\% of the vertices clustered due to convergence of multiple dendrites into single sites of up to $35 \mu \mathrm{m} \mathrm{m}$ in diameter. Such clusters were rare in random simulations of network formation. While clustering had a minor effect on $L$ and $C$ in graphs including only dendrites, it significantly affected these parameters when we added new edges that simulated axonal growing. Such axonal behavior was observed in the culture where it led to accumulation of synaptic connections at the clusters. Hence, multiple-dendritic convergence leads to synaptic clustering and may serve as a mechanism of plasticity since it was found to be activity-dependent. 
Pattern generating circuitry and sacrocaudal afferent pathways are differentially modulated by exogeneous opioids in spinal cords of neonatal rats

Blivis D. and Lev-Tov A.

Dept. of Anatomy \& Cell Biology, The Hebrew University

Medical School, Jerusalem

The possible involvement of nociceptive pathways in generation of rhythmic patterns and the modulation of these patterns by activation of opioid receptors was examined in the isolated spinal cord of the neonatal rat. Coordinated motor activity can be induced in the isolated spinal cord by bath applied neurochemicals and by stimulation of sacrocaudal afferent (SCA). In the present work we show that the rhythm induced by stimulation of SCA at various intensities is completely blocked by bath application of morphine, or the mu-opioid receptor agonist DAMGO, but not by the delta-opioid receptor agonist SNC80. Addition of the opioid receptor antagonist naloxone $(1 \mu \mathrm{M})$ restored the rhythmic activity within 5-10 minutes. By contrast, bath application of 5HT/NMDA or alphal-adrenoceptor agonist methoxamine, induced a robust rhythmic activity in the thoracolumbar and sacrococcygeal cord in the presence of morphine or DAMGO. This rhythm was characterized by a slower cycle time compared to the untreated control preparations and by a regular alternating pattern. It is suggested that opioid receptors may be used as potent modulators of motor rhythmic patterns, that nociceptive pathways may play a role in pattern generation but that the pattern generating circuitry of the thoracolumbar and sacrococcygeal segments can be activated effectively even when the SCA pathway-interneurons are blocked by bath applied opioids.

\section{Organophosphate-induced brain damage in rats:}

\section{Alterations of protein kinase $C$ isozymes}

Bloch-Shilderman E., Gilat E., Kadar T. and Levy A.

Dept. of Pharmacology, Israel Institute for Biological

Research, Ness Ziona

Organophosphates (OP) produce robust convulsions and subsequent brain damage. They are potent inhibitors of cholinesterase (ChE), which activate cholinergic mechanisms, leading to extensive excitation of the glutamatergic system. This excitation is essential for the maintenance of convulsions and for the associated brain pathology. It has been shown that soman an irreversible ChE inhibitor, increased the level of inositol phosphate and $\mathrm{IP}_{3}$, and thus might interfere with calcium homeostasis and protein kinase $\mathrm{C}$ (PKC) activities in rat brain. PKC signaling pathway has been associated with modulation of motor behavior, learning and memory, all of which are severely impaired in OP intoxication. Nevertheless, the role of PKC in OP intoxication is largely unknown. Thus we characterized the alterations in brain $\mathrm{PKC}$ isozymes in rat hippocampus and frontal cortex following sarin $\left(1 \times L D_{50}\right)$ exposure, and evaluated the neuroprotective effect of selective peptide regulators for PKCs after such insult. The results indicated that: 1) A significant down-regulation (up to $50 \%$ of control) of membrane cPKC immunoreactivity was observed from $30 \mathrm{~min}$ up to 5 days post sarin exposure in frontal cortex. 2) An up-regulation $(45-85 \%)$ of membrane cPKC immunoreactivity was found 10 days after sarin exposure in hippocampus. 3) A significant reduction in expression of the non-conventional zeta-PKC was seen in frontal cortex up to 24 hrs post sarin. This was in contrast to the increase in expression seen in the hippocampus. 4) betall-PKC peptide (380 ng/kg), which inhibits PKC translocation, and thus activation, attenuated convulsion related neuropathology induced by sarin. This is the first report identifying a role for both conventional and non conventional PKC isozymes in OP exposure in the rat, suggesting that betall-PKC, as well as zeta-PKC, might be involved in the functional and or structural modifications in the brain that occur after OP-induced convulsions.

\section{A new neurobiological theory on primate (man and} monkey) posterior parietal (IPL) function Blum B. and Israeli

Dept. of Physiology \& Pharmacology ${ }^{l}$ and Dept. of Neurology, Beilinson Medical Center, Sackler School of Medicine, Tel Aviv University, Tel Aviv 69978

We proposed (ECVP, 2002) primate IPL feedback control of 'state of body' in external and internal environment. In support microelectrode recordings in awake monkey operant behavior hand reaching to targets HR), elucidate IPL role as reward-oriented behavior in extrapersonal space (EPS); entitling IPL to multimodality, it's neurons to multi duty encoding of monkey's and trainer's hand movements in monkey EPS. In visual and non visual HR, IPL multi-duty parametric summation is revealed; salience of visual component is hinted by it's absence when reaching is out of cell's receptive field (r.f). Tactile cells of PF show same cell r.f. linkage of tip of fingers and palm of hand, coherent with grip behavior, \& with construing body scheme. IPL neurons "learn" to respond to stimuli, de novo, in course of experience. "Touch response" is to "learnt", none to "unlearnt target" suggesting involvement of attentional gating. IPL control also over internal environment is proposed on account of parietal lesion cases reported to show body scheme disturbances "feeling unreal"; we report "as if" cardiac and/ or gastric disturbances, not verified as organic but linked to anxiety and to a loss of feeling of well being. It is proposed that such a feeling', a biological parameter aimed at homeostasis, is operative in IPL mechanisms for holistic whole-body-state awareness; it's reduction releasing a corrective action moment Possibly parietal interactions with autonomic pathways mediate this internal environment feedback control of 'whole body' physiologic state, an interaction presumably decoupled in parietal lesion cases.

Modeling connectivity patterns of the primary visual cortex from spontaneous neuronal activity and feature maps

Blumenfeld B., Bibitchkov D., Naaman S. and Tsodyks M

Dept. of Neurobiology, Weizmann Institute of Science,

Rehovot 76100

Population activity across the surface of the primary visual cortex exhibits well-known regular patterns. The location and the shape of activity patches depend on features of the stimulus such as orientation. Recent studies have shown that activity patterns generated spontaneously are similar to those evoked by different orientations of a moving grating stimulus [Kenet, et. al., Nature 2003]. This suggests the existence of intrinsic preferred states of the cortical network in this area of the brain. We deduce possible connections in such a network from a set of single condition orientation maps obtained by voltage-sensitive dye imaging. We assume the maps as attractor states of a recurrent neural network and model the connectivity using a modified version of the pseudo-inverse rule of the Hopfield network. The results suggest a local distance-dependent Mexican-hat shaped connectivity. Long-range connections also exist and depend mainly on the difference in orientation selectivity of the connected pixels. The strength of connections correlates strongly with orientation selectivity of the neurons. The dependence of the obtained synaptic weights on the distance between neurons correlates with the pattern of correlations in the spontaneous activity, suggesting that intrinsic connectivity in neuronal networks in this area of the brain underlies the activity in both spontancous and evoked reimes.

Early traumatic experience alters metabolic brain activity in the rodent limbic system

Bock J. and Braun K

Dept. Zoology/Developmental Neurobiology, Otto von

Guericke University, 39118 Magdelurg, Germany.

How does the juvenile brain respond to acute emotional stress? Early deprivation and traumatic experiences can interfere with the establishment and maintenance of synaptic networks in the developing brain. Based on a previous study we applied the 2-fluorodesoxyglucose (2FDG)-technique to look for changes in metabolic activity of thalamic, hypothalamic and prefrontal cortical brain areas during stress in 8 day old Octodon degus pups. Metabolic activation, densitometrically measured as 2-FDG uptake, was compared between four experimental groups: i) pubs, that were left undisturbed with their parents in the home cage, ii) pups, that were separated as a group of littermates iii) pups, that were individually separated for 45 minutes from the parents, iv) pups, treated as group iii but during separation exposed to maternal vocalizations. We found significant alterations of metabolic brain activity in all separated groups in thalamic and hypothalamic brain areas and in the orbitofrontal cortex. In general, a strong reduction of brain activity was seen in the separated animals compared to the pups with parents. However, there were also differences between the animals of the three separated groups. The individually isolated animals (group i) showed a more pronounced reduction of 2-FDG uptake than the degu pups separated together with their littermates (group ii) and the degu pups stimulated with maternal vocalizations during separation (group IV). Since neuronal activation regulates the development of synapses during postnatal brain maturation, the disturbed activation during stressful situations may interfere with the refinement of limbic connectivity patterns, resulting in altered processing of emotional stimuli in adulthood

Supported by SFB 426/DFG. 
Deciphering the genetic program undelying transmitter phenotype in $P 19$ developing neurons Bogoch Y. Tayar S. and Linial M.

Dept. of Biological Sciences, Institute of Life sciences, The Hebrew University, Jerusalem

The generation of a specialized neuron relies on induction of an intrinsic gene regulation program and on responding to environmental cues. P19 cells are mouse originated embryonal cells that serve as a model for studying differentiation processes. Neuronal induction is performed by aggregating the cells to embryonic bodies like structures in the presence of low concentration of retinoic acid for 4 days. The potential of these cells to mature and efficiently release neurotransmitter (NT) was established in our lab. We discovered that several variables, most notably, cell density and various neurotrophic factors, most notably NGF and CNTF affects neuronal maturation, survival and most surprisingly, the choice of NT phenotype. Upon plating the cells at a low cell density (Sparse) a cholinergic phenotype dominates the culture while the same culture when plated at high density (Dense) becomes primarily glutamatergic. We hypothesized that key genes that determine the phenotypic identity should be under a strict regulation and furthermore that the acquisition of a phenotype is an early event of neuronal differentiation. A differential analysis of a large number of genes allows us a global view of the processes leading to phenotype determination. Results from several Affymetrix microarray experiments (12,000 genes/array) were analyzed using advanced bioinformatical methods. Only $2.5 \%$ of the genes tested were differentially expressed between the Sparse and Dense culture. Individual genes $(\sim 10)$ that whose expression was maximally changed as well as a set of genes that share a common biological pathway were selected for further investigation. One set of such pathway-related genes is the Nedd4A, Uba3 and Cullins that are part of the SCF ubiquitination process. By manipulating the physiological state of the cells, we are able to change the Cullin expression level while other candidate genes are unaffected.

We suggest that Cullins and specifically Cullin 3 is a key factor in linking gene expression and protein degradation by changing the balance of the two conflicting regulatory mechanism in early differentiation. The involvement of additional genes such as doublecortin and several of the genes that are involved in Wnt and $\beta$-catenin pathway are discussed.

Analysis of the effects of oxidative stress and potential neuroprotective agents in early differentiating neurons Boutboul $\mathrm{O}^{1,2},{ }^{1}$, Bledi $\mathrm{Y}^{1}{ }^{1}$, Weinstock M. ${ }^{2}$ and Linial M. ${ }^{1}$ 'Dept. of Biological Sciences, Institute of Life Sciences and ${ }^{2}$ Dept. of Pharmacology, Faculty of Medicine, Hebrew

University, Jerusalem

Oxidative stress is a pivotal factor in neuronal degeneration. Following trauma, damaged tissue liberates large amounts of reactive oxygen species (ROS). These can cause lipid peroxidation, DNA damage, protein modification and denaturation. We have examined the conditions for inducing oxidative damage in a mouse embryonic carcinoma cell line P19 that can be induced by retinoic acid to show the behavior of functional neurons that efficiently release neurotransmitters. P19 cells in the form embryonic body-like structures were treated with retinoic acid for 4 days to induce terminal differentiated neurons. Following trypsinization and mechanical disruption, the derived cells were plated and rapidly extended neurites, forming a layer of highly connected neuronal networks. To induce ROS we exposed the cells to $\mathrm{H}_{2} \mathrm{O}_{2}(5-200 \mu \mathrm{M})$. Cell damage was quantified using propidium iodide (PI), an indicator for membrane integrity, JC-1, a probe of mitochondrial membrane potential and Âlamar Blue, a redox fluorescent indicator of mitochondrial metabolic function. Neuronally-differentiated cells showed a dose-related cytotoxicity with $\mathrm{H}_{2} \mathrm{O}_{2}(4-16 \mu \mathrm{M})$. Approximately $80 \%$ cells lost their membrane potential within $40-60$ min of exposure to $15 \mu \mathrm{M}$. The damage was irreversible as $30 \mathrm{~min}$ later the membrane was fully permeable to PI. Pretreatment with TV3326, $(0.1-1 \mu \mathrm{M})$ a propargylamine-containing cholinesterase inhibitor significantly decreased the cholinesterase inhibitor significantly decreased the
ROS-induced cytotoxicity induced by $4-8 \mu \mathrm{M} \mathrm{H}_{2} \mathrm{O}_{2}$, but not by $16 \mu \mathrm{M}$ or more. The mechanism of protection is currently being studied by the application of specific markers of early apoptosis such as Annexin V and DNA fragmentation dyes We conclude that neuronally-differentiated P19 cells are a suitable model for studying the mechanisms underlying ROC-induced damage and potential neuroprotective agents.
Salt appetite is not dependent upon taste-related sensory variables

Bracha A. Portnoy F., Zach G. and Leshem M

Dept. of Psychology and Brain \& Behavior Center. University of Haifa

Genetic sensitivity to the taste of PROP, lingual papilla density, and intensity and hedonics of basic tastes, have been postulated to influence dietary intake and BMI. It is not known if these variables also determine salt appetitc. PROP taster status, intensity and hedonics of four tastes $(\mathrm{NaCl}$, sucrose. citric acid and quinine $\mathrm{HCl}$ ) density of anterior lingual fungiform papilla, dietary and activity questionnaires and measures of salt appetite were examined in 82 students. PROP responses correlated with hedonics of $\mathrm{NaCl}$, and correlated negatively with hedonics of sucrose. No relationships were found between PROP sensitivity, density of papilla, and responses to citric acid or quinine, and none of these variables predicted salt appetite, dietary intake or BMI. However, salt appetite correlated with level of physical activity. Since salt appetite has been related to exercise, our findings may support the notion that salt intake is influenced by physiological need rather than taste sensory variables.

Supported by ISF Grant 902/00-2 to Micah Leshem

Modeling human eye injury on psychophysical and perceptual performance using simulated scotomas Brandeis R. ${ }^{2}$ Peri D. ${ }^{1}$, Egoz I. ${ }^{1},{ }^{2}$ Sapiens N. ${ }^{2}$ and Turetz J. 'Dept. of Pharmacology, Israel Institute for Biological Research, Ness Ziona: ${ }^{2}$ El-Op, Ness Ziona; ${ }^{3}$ Dept. of Ophthalmology. Assaf Harofeh Medical Center. Zerifin

Macular scotomas, affecting visual functioning, characterize many eye and neurological diseases like AMD (Acquired Macular Degeneration), diabetes mellitus, multiple sclerosis and others. In this work, foveal and parafoveal visual field defects were modeled, and their effects were evaluated on spatial contrast sensitivity and target recognition performance. The modeled occluding scotomas, of different size, were superimposed on the target stimuli presented on the computer display, and were stabilized on the retina using a mono Purkinje Eye-Tracker. Spatial contrast sensitivity was evaluated using square-wave grating stimuli, whose contrast thresholds were measured using the method of constant stimuli with "catch trials". In the target recognition task the visual search display was conjunctive, the targets of which shared three features: size, contrast and position. Search accuracy as well as R.T. measures were used for performance evaluation. The artificial scotomas suppressed spatial contrast sensitivity in a size dependent manner, similar to previous studies. Deprivation effect was dependent on spatial frequency, consistent with retinal inhomogeneity models. Target recognition was impaired as a function of size and retinal location of the artificially generated scotomas. The data suggest that modeling retinal damage, simulating visual field deficits, is an efficient method for estimating the relationship between retinal lesion characteristics and visual functioning.

Asynchrony between visual and auditory modalities as a possible explanation of dyslexia

Breznitz Z

Laboratory for Neurocognitive Research. Brain and Behavior

Center. University of Haifo

A theory is proposed suggesting that asynchrony between the processing rates of the visual and auditory modalities, and stages of brain activity may be an underlying cause of dyslexia. This research project investigated whether asynchrony of speed of processing (SOP) between the visual-orthographic and auditory-phonological modalities, can account for word recognition deficits among dyslexic readers. SOP among adult developmental dvslexic readers was compared to that of chronologically age-matched regular readers. SOP was assessed using nonlinguistic and linguistic auditory and visual low-level tasks, and higher-level lexical decision orthographic and phonological tasks. Behavioral and electrophysiological (ERP and LORETA ) measures were obtained. Data indicated that dyslexic readers were significantly slower than controls on most of the experimental tasks. Moreover, dyslexics revealed a systematic SOP gap between the auditory-phonological and visual-orthographic modalities. This gap was found in both P200 and P300 latencies. Among dyslexic as compared to normal readers data also revealed a larger time gap between P200 and P300 components. Data analysis revealed that the between modalities gap scores, and the processing time gap between P200 and $\mathrm{P} 300$ explained most of the variance in word recognition. Significant differences were also found between the two groups of subjects in the intensity and in the localization sources of brain activity. On most of the experimental tasks in the control group brain activity was more intense and involved activation in more areas of the brain as compared to the dyslexic group. 
Brain activity of regular and dyslexic readers while reading Hebrew as compared to English sentences Breznitz Z., Oren R. and Miller-Shaul S.

Laboratory for Neurocognitive Research, Brain and Behavior Center, University of Haifa

The aim of the present study was to examine differences among 'regular' and dyslexic adult bilingual readers when processing reading and reading related skills in their first (L1 Hebrew) and second (L2 English) languages. Brain activity during reading Hebrew and English unexpected sentence endings was also studied. Behavioral and electrophysiological measures including Event-Related Potentials (ERP) and LORETA methodology were employed. Results indicated discrepancies in the processing profiles of dyslexic and regular bilingual readers in both first and second languages. In general, the amplitudes of the evoked potentials were higher and the latencies longer among dyslexic readers during processing of information in first and second languages (L1 and L2), but were more pronounced in English (L2).

LORETA (Pascual-Marqui et al., 1994) analysis indicated evidence that the source of brain activity measured by current density of brain activation is different when reading Hebrew as compared to English sentences mainly among dyslexics and not among regular readers. The date from the present study supports the "dominant bilingual' hypothesis for defining bilinguals. A discrepancy between achievement in performing various $\mathrm{L} 1$ and $\mathrm{L} 2$ tasks was consistent across groups. Both groups were better in there mother tongue, which was Hebrew as compared to English.

AHP reduction after odor learning is caused by reduction in $\mathrm{SI}_{\mathrm{AHP}}$, but not by expression changess of SK channels Brosh I. ${ }^{2}$, Rosenblum K. ${ }^{2}$ and Barkai E.

${ }^{7}$ Zlotowski center for neuroscience, Ben-Gurion University, Beer-Sheva; ${ }^{2}$ Center for Brain and Behavior, Haifa University We have previously shown that learning-induced enhancement in neuronal excitability is mediated by reduction in one or more of the calcium-dependent potassium currents that control neuronal frequency adaptation. The purpose of the present research was to identify which of these currents is reduced after learning. Rats were trained in an olfactory discrimination task to distinguish between pairs of odors until demonstration of rule learning. Two days after training completion, intracellular recordings were made from pyramidal neurons in layer II of piriform cortex brain slices. As previously shown, AHP amplitude was significantly smaller in neurons from trained rats compared with neurons from pseudo trained and naive rats $(-4.98+0.30 n=32$ trained, $-6.79+0.38 n=23$ pseudo trained, $-6.76 \pm 0.37 \mathrm{n}=24$ nalve). In the presence of specific $\mathrm{I}_{\text {AHP }}$ blocker apamin (50nM), AHP amplitude remained significantly smaller in neurons from trained rats $(-2.76+0.41$ $n=20$ trained, $-4.67+0.59 n=16$ pseudo trained, $-4.73+0.3 \overline{0} n=9$ naive), and the reduction in AHP amplitude was similar in all three groups. In order to test the possibility that changes in SK1, 2 and 3 channels expression is mediating the reduction in AHP, we used western blot analysis. Expression of SK1, 2 and 3 did not change after training. In the presence of noradrenaline $(10 \mathrm{mM})$ which blocks the $\mathrm{sI}_{\mathrm{AHP}}$ and increases the $I_{A H P}$, AHP amplitude was similar in neurons from all groups $(-6.32 \pm 0.71 \quad n=14$ trained, $-5.20 \pm 0.54 n=12$ pseudo trained, $-5.64+0.40 n=24$ naive). These results indicate that the reduction in AHP amplitude after learning is due to secondary messengers affecting the slow $I_{A H P}$ and not to SK channels modulation of expression.

Effects of chronic oral homocysteine on mice behavior Buskila E. and Levine J.

Ben Gurion University, Beer Sheva

Homocysteine is an amino acid not included in protein synthesis. It participates in the single carbon cycle that provides methyl groups to multiple metabolic functions in the body. It is present normally in human plasma in very low concentrations being converted enzymatically rapidly to methionine. A recent study found highly elevated plasma levels of homocysteine in patients with schizophrenia and this study was replicated in an independent sample. Homocysteine has been shown to activate apoptotic processes via NMDA receptors and elevated homocysteine in schizophrenia could therefore be a mechanism for the cognitive deterioration known to occur in schizophrenia. We therefore decided to try to develop a model of homocysteine neurotoxicity in mice

Mice were fed homocysteine in water at a concentration of $200 \mathrm{mg} / \mathrm{kg}$ mouse (calculated final dose) Water was changed daily. Independent samples of animals were studied at 1 month, 2 months, 3 months, 4 months, 5 months and 6 months for behavioral tests having face value similarities to defects in human schizophrenia such as pre-pulse inhibition, visual memory in the Morris Water Maze, apomorphine induced sterotopy, and roto rod neurological integrity. Animals were sacrificed after behavioral testing and carotid blood taken for homocysteine levels. Homocysteine levels were elevated between 300 and $400 \%$ by this procedure. No homocysteine induced defects were found in any behavioral test until month 5 when a mild but statistically significant abnormality in the Morris Water Maze was detected.

\section{Effect of chronic D9-THC on gene expression in the rat brain}

Butovsky E., Goncharov I., Zangen A., Juknat A., Elbaz J., Nevo I. and Vogel Z

Dept. of Neurobiology, Weizmann Institute of Sceince, Rehovot The use of cannabinoids (most notably D'-THC, the active component in marijuana) has been widespread throughout history. Cannabinoids have a multitude of physiological and therapeutic properties, but are especially known for their rewarding effects. Several brain regions have been implicated in the rewarding effects of cannabinoids, and in the process of addiction to drugs of abuse. There is also evidence that cannabinoid treatment can affect gene expression. The advent of the DNA microarray allows the analysis of thousands of genes in parallel. We have used the Affymetrix GeneChip System to identify the changes in gene expression induced by $D^{3}$-THC $(1.5 \mathrm{mg} / \mathrm{kg} /$ day i.p. for 7 days $)$ in specific regions of the rat brain, which have been shown to play a role in cannabinoid reward (such as the nucleus accumbens). The expression of 60 " genes was found to be affected by more than 2 fold by this treatment, with most of these genes (50") having been upregulated, while only a few were downregulated. Among the upregulated genes were various receptors, channels, neurotrophic and growth factors, and molecules related to apoptosis cytoskeleton, and cell adhesion. The results for genes found to be of interest have been corroborated by other methods (such as quantitative real-time PCR). Subsequently, we used various immunological approaches (immunohisto-chemistry, Western blotting) to follow the changes in expression of some of these genes. This study should advance our understanding of the genes regulated by cannabinoid treatment, and of the role of their gene products in regulating the rewarding properties of the cannabinoids, and will eventually lead to a better understanding of the adaptive changes that take place during chronic exposure to these widely abused drugs.

Supported by the Nella and Leon Benoziyo Center for the Neurosciences

Aggregated $\beta$-amyloid mimics bacterial behavior by rendering microglia cytotoxic phenotype and impairing antigen presentation: Implications for Alzheimer's disease Butovsky O., Talpalar A.E. and Schwartz M.

Dept. of Neurobiology, Weizmann Institute of Science, Rehovot Inflammation has often been blamed for deleterious effect in the central nervous system (CNS). Using rat microglia and organotypic hippocampal slice cultures we show here that microglia activated by aggregated $\beta$-amyloid or lipopolysaccharide (LPS) are detrimental to neural tissue. In contrast, interferon (IFN) $-\gamma$ rendered microglia a protective phenotype to neural tissue. In addition, expression of cell-surface class II major histocompatibility complex antigens (MHC-II) was impaired in detrimental microglia. The impairment occurred at the level of expression of mRNA encoding the invariant chain (Ii, a key participant in the presentation by MHC-II of small peptides to helper CD4+ lymphocytes). The results suggest that self-agents which play a role in neurodegenerative diseases, like invading microorganisms, induce microglia to adopt a neurotoxic phenotype that evades, at least transiently, the adaptive immune mechanism needed to maintain the CNS by fighting off toxic self-compounds.

Isoforms specific effects of ApoE on lipid uptake and synthesis and on neurite outgrowth and synaptogenesis Caspi L., Grundland A., Ashery U., Devir A. and Michaelson DM.

Dep. Neurobiochemistry, Tel Aviv University, Tel Aviv

Apolipoprotein $E$ (apoE), is the major brain lipoprotein. There are three apoE isoforms (apoE2, apoE3, apoE4) of which apoE4 is a main risk factor of Alzheimer's disease. In the present study we investigated the possibility that apoE affects neuronal lipid metabolism and synaptogenesis in an isoform-specific manner. In the presence of Intralipid (an exougenous lipid solution), ApoE3 inhibited lipid uptake and increased lipid synthesis by Neuroblastoma $\mathrm{N} 2 \mathrm{a}$, whereas apoE4 had no such effects. ApoE4 binds more avidly to Intralipid than apoE3 but both bind similarly to N2a cells. This suggests that the isoform specific effect of apoE3 and apoE4 on lipid uptake and synthesis may be related in part to 
differences in apoE binding to N2a cells and to the extent of apoE lipidation. Morphological experiments revealed that apoE4 inhibited neurites outwrowth compared to apoE3 and control. The finding that ApoE3 enhanced neurites outgrowth and inhibited lipid uptake suggests that factors additional to lipid uptake may mediate the effect on neurites outwrowth. In order to learn about possible differential effects on synapses' formation and function, we established a system of cultured hippocampal neurons derived from ApoE deficient mice. The neurons are exposed to Glia Condition Medium obtained from transgenic mice expressing human ApoE3 or ApoE4 and neurites elongation, synapse formation and synaptic transmission are measured using electrophysiology and Immunocytochemistry. The effects of ApoE isoforms on synapse formation and function is currently under investigation.

Expression of genes and proteins in rat frontal cortex in response to chronic administration of haloperidol plus fluvoxamine versus clozapine: Differential effects on GABA system

Chertkow Y ${ }^{1}$ Weinreb $\mathrm{O}^{1}$, Youdim $\mathrm{MBH}^{1}{ }^{1}$ and Silver $\mathrm{H}^{2}{ }^{2}$ Eve Topf \& U'S National Parkinson's Foundation Centers of Excellence for Neurodegenerative diseases, Rappaport Faculty of Medicine, Technion, Haifa; ${ }^{2}$ Molecular Neuropsychiatry Unit, Shaar Menashe MHC Brain Behavior Laboratory.

Faculty of medicine Technion

Recent research has shown that 'negative symptoms' of schizophrenia, which do not respond to standard antipsychotics, can be improved by adding serotonin selective reuptake inhibitor (SSRI) to the treatment. The molecular and biochemical mechanisms underlying the efficacy of the combined treatment have not been studied. We proposed that chronic treatment with SSRI combined with typical antipsychotics produces a 'net effect', different from that of the individual drugs and common to that of the atypical antipsychotic, clozapine which also ameliorates negative symptoms. The purpose of this study was to identify, in the rat frontal cortex, gene and protein cascades whose expression is altered by treatments, which ameliorate negative symptoms. cDNA arrays and real-time PCR were used to identify and validate gene expression alterations following 30 days administration of haloperidol $(1 \mathrm{mg} / \mathrm{kg})$, fluvoxamine $(11 \mathrm{mg} / \mathrm{kg})$, combination of fluvoxamine and haloperidol, clozapine $(1 \mathrm{lmg} / \mathrm{kg})$ and saline. Genes of interest were also examined at the protein level by using the western-blotting technique. We found that the combined treatment affected gene and protein expression differently than fluvoxamine or haloperidol alone despite similar drug concentrations in the brain. Some of the changes unique to combined treatment were similar to those following clozapine administration. Notable was the differential effect on GAD67 and PKCb proteins that were increased following treatment with haloperidol, but not after combined treatment or clozapine. These results suggest that gene and protein candidates from the GABAergic system may be involved in mechanisms alleviating negative symptoms. Effective treatments may act by reducing the "net" relative overactivity of the GABA system in the frontal cortex, which may characterize schizophrenia.

Do olivary subthreshold oscillations mind the gap?

Chorev E. , De Zeeuw C.I. ${ }^{2}$, Manor Y. ${ }^{3}$, Sohl G. ${ }^{\text {, }}$, Willecke $\mathrm{K}^{4}$ and Yarom $\mathrm{Y}^{1}$

${ }^{3}$ Dept. of Neurobiology, Life Sciences, Hebrew University, Jerusalem, Israel: ${ }^{2}$ Dept. of Neuroscience, Medical Faculty Erasmus MC, 3000DR Rotterdam, The Netherlands; ${ }^{3}$ Dept. of life sciences \& Zlotowski Cntr for Neuroscience, Ben-Gurion U., Beer-Sheva. Israel; ${ }^{4}$ Institute of Genetics, Division of Molecular Genetics, University of Bonn, 53117, Germany Neurons in the olivary nuclei exhibit subthreshold oscillations (STOs) of their membrane potential. Many evidences support the hypothesis that gap-junctions between olivary neurons are essential for the generation of STOs. To test this hypothesis, we used a strain of knockout mice that lack Connexin 36 (KO $\mathrm{Cx} 36$ ), the protein that forms gap junctions in the olive and other brain areas. Except for small deficits in vestibuloocular reflex (VOR) gain learning; the phenotype of $\mathrm{KO} \mathrm{Cx} 36$ appears normal. Furthermore, despite the absence of electrotonic coupling, neurons from $\mathrm{KO} \mathrm{Cx} 36$ mice maintained the ability to oscillate. However in contrast to the STO in WT neurons, the STO in KO neurons were voltage dependent. Hence, knocking out the coupling transforms quiescent olivary neurons into oscillatory neurons. This transformation involves an increase in input resistance, due to higher specific membrane resistance, and an increase in the low-threshold calcium conductance. Using the dynamic clamp, we demonstrated that these changes in membrane properties are sufficient to explain the transformation of a quiescent WT neuron into a KO-like neuron. These results suggest that the lack of $\mathrm{Cx} 36$ proteins triggers compensatory mechanisms, which transform the olivary neurons from quiescent to auto-rhythmic. Further support for this hypothesis comes from developmental studies, which show that the differences between WT and KO emerge during the maturation of the olivo-cerebellar system. The voltage dependence of these oscillations provides a mean to control their appearance: by a common inhibitory input for instance. We propose that the ability of olivary neurons to produce STOs using different strategies call attention to the importance of these oscillations to the normal function of the animal.

Experimental analysis of parameters determining the electrical coupling between neurons and transistors Cohen $A^{1}$. Shappir $J^{2}$, Yitzhaiek $S^{3}$ and Spira M.E. ${ }^{1}$ Depts. of Neurobiology, ${ }^{2}$ Engeneering, and ${ }^{3}$ Chemistry The Hebrew University of Jerusalem.

The use of field effect transistors (FETs) to detect action potentials generated by cultured neurons, progressed in recen years. The recorded signals are nevertheless highly variable in shapes and amplitudes

Using isolated Aplysia neurons and FETs produced by us we analyzed the parameters that determine the shape and amplitude of the FETs output signals

We found that the critical parameters for recording action potentials by FETs are the generation of current flow across seal resistance $\left(R_{s}\right)$ formed between the FET sensing area and the solution around it. Current flow across $R_{s}$ is generated under two conditions (a) The formation of a voltage drop along a non-isopotential neuron with a space constant significantly larger than the dimensions of the sensing area. Or (b), polar distribution of the ionic channels that generate action potentials in isopotential neurons. These principals were experimentally demonstrated. For example, The FET's recordings of propagating action potentials generated by a non-isopotential neuron with a long axon are abolished when the axon is transected, and the neuron continues to generate action potentials but becomes isopotential. Another parameter is the values of $R_{s}$ and the currents generated across it. To assess the contribution of $R_{s}$ to the shape and amplitude of action potentials recorded by FETs, we mechanically forced the cell body or an axonal segment against the sensing area of a FET, while injecting different forms of current pulses into the neuron. The resulting voltage drop across the neuron's membrane and the potentials were recorded by the FET. This allowed us to calculate the seal resistances produced by the mechanical pressure, and to relate it to the amplitude and shapes of the voltage outputs and action potentials of the FETs. The insights gained by this study simplify the interpretation of recorded signals by transistor and enables us to designing more sophisticated neuron-transistor junction.

Acetylcholine modulates dentate gyrus neurogenesis in the

Cohen J.E. ${ }^{1}$, Dori A. ${ }^{2,3}$, Friedman A. ${ }^{1}$ and Soreq $\mathrm{H}^{3}$

Zohotowski Center of Neuroscience, Ben-Gurion University,

Beer Sheva, ${ }^{2}$ Neurosurgery, Soroka MC, Beer Sheva;

${ }^{3}$ Biological Chemistry, Hebrew University of Jerusalem

In the adult mammalian dentate gyrus (DG), neurogenesis proceeds continuously, producing functional granule cells with pivotal roles in learning and memory. Both memory and stress, which involve cholinergic neurotransmission, influence DG neurogenesis. To test the possibility that the morphogenically active acetylcholine (ACh) hydrolyzing enzyme acetylcholinesterase (AChE) participates in DG neurogenesis, we employed fluorescent in-situ hybridization. neurogenesis, we employed fluorescent in-situ hybridization.
Proliferating cells at the sub-granular layer (SGL) were intensely positive for AChE mRNA. Furthermore, DG cells intensely positive for AChE mRNA were immunolabeled for TUJ1 (all tubulin) but not NeuN (Neuron specific nuclear protein), identifying them as immature granule neurons. To quantify the AChE effect on these cells, mice were injected i.p. with Bromo-deoxy-Uridine (BrdU) and sacrificed 3 weeks post-injection. Proliferating cells in the SGL, positive for the proliferating cell nuclear antigen (PCNA), and surviving and the ratio of GCL BrdU ${ }^{+}$cells to SGL PCNA cells was defined as the "survival index". TgSin mice, overexpressing catalytically inactive "synaptic" AChE, presented a significant $(\mathrm{P}<0.01)$ increase in SGL proliferation compared to either FVB/N controls, TgS transgenics overexpressing catalytically active "synaptic" AChE or TgR transgenics with excess "readthrough" AChE. In contrast, TeS mice showed a higher number of surviving cells $(P<0.05)$ compared with $F V B / N$ controls. Also, brain AChE hydrolytic activity, which was lowest in TgSin and highest in TgS mice, was associated with the survival index of GCL neurons. Our findings support the notion that $\mathrm{ACh}$ induces proliferation and reduces survival in DG neurogenesis, whereas $\mathrm{AChE}$ promotes survival of maturing GCL neurons. 
Reconstitution of voltage-dependent exocytosis in xenopus laevis oocytes

Cohen R and Atlas D

Dept. of Biological Chemistry, The Hebrew University of

Jerusalem, Jerusalem 91904

Voltage dependent exocytosis is a complex mechanism, assembled by several steps that are carried out by various proteins. Some of these proteins are essential for the final steps of vesicle fusion while others play a modulatory function. In the present study we suggest to determine the minimal set of proteins that is essential for triggering depolarization evoked secretion. For that purpose we suggest to reconstitute the process of secretion in Xenopus oocytes and monitor changes in capacitance directly as a measure of exocytosis. Xenopus Laevis oocytes are broadly used as a heterologous expressing system to study the function and activity of proteins such as ion channels, transporters and receptors. Previously we showed interactions between voltage-gated calcium channel and synaptic proteins $(1,2,3)$ and here we monitor changes in capacitance that result from the fusion of oocytes vesicles in response to depolarization. Total membrane capacitance is a known technique used to determine changes in membrane surface as a measure for endo/exocytosis processes. We employ the "Paired Ramp" technique for whole cell capacitance to monitor online analysis of exocytosis events in the Xenopus oocytes according to Schmitt et al., 2002 (4). We show that co-expression of synaptic proteins implicated in exocytosis along with voltage gated calcium channels is sufficient for induction voltage-dependent vesicle fusion as revealed by whole cell $\mathrm{Cm}$ changes. Our data demonstrates that the Xenopus oocyte system can be used to faithfully mimic voltage-dependent exocytosis. We propose a stepwise reconstituting of the exocytotic machinery from its elements to further understand the role of each component in this complex process

1. Cohen R, Elferink LA Atlas D. (2003) JBC 278(11):9258-66; 2. Wiser O, Cohen R, Atlas D. (1999) PNAS U S A. 99(6):3968-73; 3. Wiser O, Bennett MK, Atlas D. (1996) EMBO J. 15(16):4100-10; 4. Schmitt BM, Koepsell (2002) Biophysical J. 82:1345-1357

ERK activation is correlated with maintenance of learning-induced enhancement of synaptic transmission Cohen-Matsliah SI ${ }^{1}$, Rosenblum $\mathrm{K}^{2}$ and Barkai ${ }^{2}$

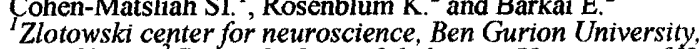
Beer Sheva; ${ }^{2}$ Center for brain \& behavior. University of Haifa We have shown previously that olfactory learning is accompanied by enhanced neuronal excitability of piriform cortex pyramidal neurons, manifested by reduced post-burst after-hyperpolarization (AHP), and enhanced synaptic transmission between these cells, manifested by reduced paired-pulse facilitation (PPF). Extra cellular regulated kinase (ERKI/II) are thought to play major role in learning and memory as well as synaptic plasticity in cortical areas. The purpose of the present study was to determine whether ERKL/I has a role is maintaining olfactory-learning induced cellular modifications. Intracellular recordings from pyramidal neurons were performed in piriform cortex brain slices. The specific MEK inhibitor PD98059 (40 לM) caused a significant increase in PPF amplitude in neurons from trained rats only Consequently, the difference in PPF amplitude between trained and pseudo trained rats was diminished (from $1.22+0.25, \mathrm{n}=2$ to $1.56+0.29 n=7$ in trained and from $134+0.20, n=6$ to $1.44+0.18, n=5$ in pseudo trained). PD98059 did not have an effect on post-burst AHP amplitude in any group on neurons (from $6.25 \pm 2.29, n=44$ to $6.53+2.72, n=19$ in trained and from $7.82+1.74, \mathrm{n}=20$ to $7.31+1.7 \overline{4}, \mathrm{n}=8$ in pseudo trained). We conclude that ERKI/II is involved with long-term modulation of synaptic transmission in the piriform cortex of trained rats but not in learning-induced enhancement of neuronal excitability.

Prosaposin increases density and glutamate receptor R1 content of spines dependent on MÂPK and synaptic

activity $_{1,2,3}$, Morales C.R ${ }^{4}$ and Baranes D. ${ }^{1,2,3}$

${ }^{1}$ Dept. of Life Sciences, Ben Gurion U.. Beer Sheva, Israel; ${ }^{2}$ Zlotowski Center for Neuroscience. Ben Gurion U., Beer

Sheva, Israel: ${ }^{3}$ The Institute for Applied Research. Ben Gurion U., Beer. Sheva, Israel; ${ }^{4}$ Dept. of Anatomy \& Cell Biology, McGill U., Montreal, Canada.

Synaptic plasticity involves strengthening of synaptic efficacy. This can occur via increasing the density of synapses or elevating the concentration of synaptic neurotransmitter receptors. We found a deficiency in the Glutamate Receptor (GluR1) subunit of the AMPA receptor in hippocampi of knockout mice for prosaposin a secreted neurotrophic factor In hippocampal cultures, inhibition of extracellular prosaposin activity with a specific antibody reduced both spine density and the level of GluRI but not of N-methyl-D-aspartate receptor 1 (NR1) in dendrites or in spines. Similarly, elevation of prosaposin activity by application of exogenous prosaposin increased the content of GluR1 but not of NR1 in spines. Inhibitors of synaptic activity and of the MAPK pathway blocked prosaposin's effects. Hence, prosaposin increases spine density and targeting of GluR1 to spines in an activity-dependent manner, suggesting that prosaposin promotes synaptic plasticity.

\section{Mean-field theory for a network of spiking neurons with} slow learning of working memory

Curti $E$. Mongillo $G$. and Amit DJ

INFM, dip. di Fisica, Universita' di Roma, La Sapienza; Racah Institute of Physics, Hebrew University, Jerusalem dip di Fisiologia Umana, Universita' di Roma, La Sapienza

Mean-field (MF) theory (a description in terms of average spike rates in homogeneous neural populations) is extended to realistic networks of excitatory and inhibitory spiking neurons embedding working memory of $p$ randomly chosen stimuli of a given coding level $f$. The underlying synaptic matrix is a result of generic, slow synaptic plasticity of two state synapses, upon repeated presentation of a fixed set of an arbitrary number of external stimuli. The theory is developed in terms of neural subpopulations each responsive to a given number of stimuli in the training set (multiplicity). The structure of the synaptic matrix is also determined by this classification1. The resulting number of MF equations would have been $2 p+1$. The effective number is reduced to $2(\mathrm{pf}) 1 / 2$ by an automatic process which produces very precise results and reduces very significantly the computational complexity.The mean-field results for the bifurcation diagrams and the rate distributions in stationary states of the network agree well with microscopic simulations of the full network of spiking neurons.

$\mathrm{N}$. Brunel, F. Carusi and S. Fusi (1998) Slow stochastic \{Hebbian\} learning of classes in recurrent neural networks, Network 9:123-152

Supported by the Israel Science Foundation (ISF) Grant $\# 8009$, and and the Center of Excellence Grant 'Statistical Mechanics and Complexity" of the INFM. Roma-1.

Salt preference, dietary intake, taste papillae, PROP response, and taste responses in Ethiopian immigrants Dally Farah R., Khair A., Shalev E., Yonas A. and Leshem M. Dept. of Psychology and Brain \& Behavior Center, University of Haifa

Sensory variables, physiological need, and cultural preferences, may influence dietary intake and BMI. To determine how these variables might contributes to salt appetite, we examined taste sensory variables (PROP (6-n-propylthiouracil response, density of anterior lingual fungiform papilla, intensity and hedonics of $\mathrm{NaCl}$, sucrose, citric acid and quinine HCL), and dietary and activity guestionnaires, in a sample of 30 Ethiopian students (13. $1 \pm 1.0 \mathrm{y}(4-22)$ in Israel). Overall, salt appetite was greater than sweet preference, salt appetite correlated with number of cigarettes/day, and there was a gender difference - preference for salt snacks was greater in men than in women. Compared to a group of native-born Caucasian students, Ethiopian students had lower dietary sodium intakes, were more sensitive to the taste of salt and sugar, preferred higher concentrations of these tastes, reported quinine as more aversive, and had a lower BMI. However, salt appetite was similar. Dietary intake of macronutrients, other than carbohydrates and sodium, was inversely related to time since immigration as was level of activity, probably accounting for the stability of BMI. No relationships were found between PROP response, papilla density, taste sensitivity or hedonics, and these variables did not predict salt appetite, dietary intake, or BMI. Thus sodium intake is stable whilst intake of other macronutrients and activity levels change over length of stay in Israel. Taken together, the findings suggest that sodium intake is largely independent of taste sensory responses and cultural preferences, and therefore may be physiologically regulated by need.

Supported by Israel Science Foundation Grant 902/00-2 to Micah Leshem.

FMRI BOLD responses elicited by sound location changes Deouell L.Y. ${ }^{1,2}$, Heller A. ${ }^{2}$, D'Esposito $\mathrm{M}^{2}$ and Knight R.T. ${ }^{I}$ The Hebrew Universtiy of Jerusalem. Israel; ${ }^{2}$ University of California, Berkeley, USA

An unattended change in the acoustic environment elicits a neural response registered as the mismatch negativity event related potential. Electric and magnetic dipole analyses suggest close but non-overlapping sources in response to changes in various attributes. However, EEG and MEG are inherently 
ill-posed to portray the full spatial extent of activity Functional MRI has been recently used to delineate active brain areas in response to deviations in frequency, duration, and phonetic structure. Responses to changes in spatial location of sound were more elusive. We used a 4T FMR scanner to measure responses to changes in virtual sound locations in 13 subjects. Outside the scanner, broad-spectrum sounds were played from speakers surrounding the subjects, and recorded using miniature microphones placed in the entrance of the ear canal of each subject. In the scanner, the sounds were played back via MRI compatible headphones Prior to scanning, subject judged the relative positions of 5 sounds positioned at $\pm 60, \pm 15$, and 0 degrees with over $90 \%$ accuracy. To prevent masking by scanner noise, we used "sparse" fMRI design - MRI scans were spaced 12 seconds apart, and sounds were presented between scans. Blocks of standards alternated with blocks of standards and deviants. In separate runs, standards and deviants were 15 and 60 degrees to the right of the subject, respectively, or vice versa (counterbalanced within subjects). Subject ignored the sounds while watching a movie. Random effect analysis contrasting standards-and-deviant blocks with standard-only blocks showed robust bilateral activation in Heschl's gyrus extending posteriorly into the planum temporale. In addition, small activated clusters were found in the left inferior parietal, right cingulate, and right middle frontal gyrus. Spatial mismatch response is generated mostly in the primary and secondary auditory cortices, with possible contribution from extra auditory loci

\section{Dissociated roles for the basolateral and lateral nuclei of} the amygdala in classical conditioning: Differential involvement in contextual and tone fear conditioning Desmedt A., Calandreau L., Decortes L. and Jaffard R Laboratoire de Neurosciences Cognitives, CNRS UMR 5106, Universit' de Bordeaux I. Talence, France.

Although the basolateral amygdala complex (BLC) is required for both elemental and contextual conditioned associations (i.e discrete conditioned stimulus (CS)-unconditional stimulus (US) and context-US association), anatomical and electrophysiological studies suggest a differential involvement of the lateral (LA) and basolateral (BLA) nuclei components of the BLC in these two kinds of associations. Using a classical fear conditioning paradigm, the present study investigated this hypothesis by comparing the effects of inactivation of either the BLA or the LA during training on subsequent conditioned freezing responses to the elemental (tone) $\mathrm{CS}$ and to the context. Results showed that inactivation of the BLA impaired conditioning to the context while sparing conditioning to the tone CS. In contrast, inactivation of the LA disrupted conditioning to the tone CS but also impaired or enhanced contextual conditioning as a function of the training protocol (CS-US unpairing vs. pairing). Therefore these findings reveal dissociated roles for these two amygdaloid nuclei in fear conditioning. They indicate a selective involvement of the BLA in contextual conditioning and suggest that, beyond its requirement for elemental CS-US associations, the LA could modulate the processing of BLA-mediated context-US associations depending on the predictability of the discrete CS for the occurrence of the US.

Dynamic imaging of brain function: Relating imaging signals to underlying physiology

Devor A., Dunn A.K., Andermann M.L., Ulbert I., Narayanan S.N., Jones S.R., Einevoll G.T., Bower J.M., Boas D.A. and Dale A.M

MGH-NMR Cntr, Harvard Medical School, Charlestown, MA

Neuronal activity changes are coupled to changes in blood flow, blood volume, and blood oxygenation, collectively referred to as the hemodynamic response. Coupling between the hemodynamic response and brain electrical activity is of a great importance for interpreting fMRI. We address this question by employing simultaneous optical and electrophysiological recordings from rat cerebral and cerebellar cortex using multiple stimulus conditions.

During reflectance imaging of intrinsic signals, the illuminating light was filtered at 6 wavelengths $(560-610 \mathrm{~nm})$ using a rotating filter wheel. The raw optical data was corrected for wavelength-dependent path length differences. These multiwavelength maps were used to calculate oxyhemoglobin (HbO) and deoxyhemoglobin ( $\mathrm{Hbr}$ ) concentration changes in the response to stimulation. During laser speckle-contrast imaging the cortex was illuminated with a diode laser $(785 \mathrm{~nm})$, and the raw speckle images were converted to blood flow maps. Using both spectral and speckle data, we calculated oxygen consumption (CMRO2) maps.

Electrophysiological recordings were performed using either single metal electrodes or laminar multi-electrodes. In the cerebrum, the electrode was placed in the center of the barrel as determined by the optical activation. In the cerebellum, the signal was mapped along crus IIA. The signals were filtered at $0.1-500 \mathrm{~Hz}$ to record population field and at $150-5000 \mathrm{~Hz}$ to record multi-unit activity.

We demonstrate a non-linear relationship between the hemodynamic signal and the underlying electrical activity. Earliest hemodynamic response gives better localization of the neuronal activity. During the positive phase of $\mathrm{Hbr}$, blood volume gives the best and blood flow the poorest localization.

\section{Neuregulin rescues PC12-ErbB4 cells from cell death}

induced by -amyloid involvement of P13K

Di Segni A., Stien R., Pinkas-Kramarski R

Dept. of Neurobiochemistry, Tel Aviv University, Tel Aviv

Neuregulins (NRGs), which are highly expressed in the nervous system, bind and activate two receptor tyrosine kinases, ErbB-3 and ErbB-4. We previously showed that NRC mediates survival of PC12-ErbB4 cells from apoptosis induced by serum deprivation, TNFa treatment or $\mathrm{H} 2 \mathrm{O} 2$. These effects of NRG are mediated by the PI3K signaling pathway. In the present study we show that NRG induces a significant protective effect from b-amyloid 25-35 peptide induced cel death. The PI3K signaling pathway mediates this effect of NRG since NRG failed to rescue cells from b-amyloid insult in the presence of the PI3K inhibitor LY294002. Furthermore, the downstream effector of $\mathrm{PI} 3 \mathrm{~K}, \mathrm{PKB} / \mathrm{AKT}$, is activated by NRG in the presence of b-amyloid and PKB/AKT activation is inhibited by LY294002. In addition, our results demonstrate that $b$-amyloid-induced cell death is reduced by expression of activated PI3K. These results suggest that PI3K dependent pathways may regulate the toxic effect of $b$-amyloid. In addition, b-amyloid induced alteration in apoptotic protein levels (elevation in $\mathrm{Bax}$ and reduction in $\mathrm{BclX}_{\mathrm{L}}$ ). NRG treatment however, induced $\mathrm{BclX}_{\mathrm{L}}$ and reduced $\mathrm{Bax}$ levels The results suggest that NRG may affect cell viability by increasing the anti-apoptotic protein $\mathrm{BclX}_{\mathrm{L}}$ levels and reducing the pro-apoptotic protein Bax levels.

Closed head injury induces up regulation of Beclin 1 at the site of injury

Diskin T. ${ }^{1}$, Erlich S. ${ }^{1}$ Tal-Or P. ${ }^{1}$, Shohami E. ${ }^{2}$ and

Pinkas-Kramarski $R^{1}$

${ }^{1}$ Dept. of Neurobiochemistry. Tel-Aviv University, Tel-Aviv; ${ }^{2}$ Dept. of Pharmacology, the Hebrew University, Jerusalem

Autophagy, a bulk degradation of sub-cellular constituents, is activated in several neurodegenerative conditions. Beclin 1, a $\mathrm{Bcl} 2$ interacting protein, was found to promote autophagy. The closed head injury model was used to investigate the possible role of Beclin in neurodegeneration. It is demonstrated that levels of Beclin-1 are dramatically increased near the site of injury. Time course analysis of Beclin expression after $\mathrm{CHI}$ show an elevation of protein levels in the cortical region at the site of injury, starting at 4 hours following injury. Elevated Beclin levels were observed up to 4 weeks after the injury

Neurons and astrocytes constitute the major population of cells with the highest Beclin levels near the site of injury. Beclin expression was not detected in activated microglia/macrophages. In addition Beclin-1 expression 4 hours, 24 hours and 3 days after injury is elevated also in CA1 and $C A 2$ regions of the hippocampus. Confocal microscopy analysis suggests that the high level of Beclin protein in part of the cells, is accompanied by DNA degradation and cell death. These findings show for the first time that Beclin may play a role in brain responses to head trauma. Overexpression of Beclin may be important for autophagy at the lesion site and may serve as a mechanism to discard injured cells and reduce damage to cells by disposing injured components.

Isoform specific effects apoE on the deposition and cleareness of brain $\beta$ amyloid following inhibition of neprylisin

Dolev I and Michaelson DM

Dept. of Neurobiochemistry, Tel Aviv University, Tel Aviv

Apolipoprotein E4 (apoE4), a major risk factor of Alzheimer's disease (AD), is associated with an earlier age of disease onset and with an increase in the number of $\beta$ Amyloid ( $\beta A$ ) containing plaques. Studies of transgenic mice, which express mutant human APP together with either human apoE4 or apoE3, which is the benign apoE isoform, revealed that $B A$ deposition is enhanced isoform specifically in aged apoE4 transgenic mice. The mechanisms underlying these effects and the extent to which they are related to either enhanced aggregation or impaired removal of $\beta \mathrm{A}$ by apoE4 are yet unknown. In the present study we inhibited the $\beta A$ degrading enzyme, neprylisin, by continuous application of thiorphan into the lateral ventricle of control and apoE transgenic mice for up to one month, and examined the resulting effects of apoE4 apoE3 and of apoE deficiency on the rates and extents of $\beta A$ deposition and on the subsequent clearance of these 
deposits. Immunohistochemical examination of the brains revealed time dependent deposition of $\beta A$ which was maximal at 1 -month and which was more than three fold higher in the apoE4 transgenic mice than in the apoE3, control and apoE deficient mice. The apoE transgenic mice also had fibrillar thioflavin-S positive deposits, whose levels were about 25 percent of the total. Examination of the brains of mice a month after the application of thiorphan was terminated revealed a decrease in the cortical and hippocampal levels of $\beta A$ deposits of the apoE3 transgenic and control mice. In contrast, the levels of hippocampal $\beta \mathrm{A}$ deposits of the apoE4 transgenic mice did not decrease whereas their corresponding cortical $\beta A$ deposits did. These findings suggest that apoE4 accelerates the deposition and slows down the dissolution of $\beta A$ isoform specifically.

Excitatory- and inhibitory-like serotonin-mediated accumbal dopamine release: Alteration in an animal mode of depression is reversed by antidepressant treatment Dremencov E. ${ }^{1}$, Herman-Gispan I. ${ }^{1}$, Kinor N. ${ }^{1}$, Gefen-Aricha $\mathrm{R}^{1}$, Weizman $Y^{1}{ }^{1}$, Blatman-Jan G. ${ }^{1}$, Overstreet D. $\mathrm{H}^{2}$ and Yadid $G^{1}$

${ }^{I}$ Faculty of Life Sciences, Bar-Ilan University, Ramat-Gan Israel; ${ }^{2}$ Dept. of Psychiatry, University of North Carolina. Chapel Hill, North Carolina, USA

The aim of the current study was to determine the pharmacological mechanism of alterations in serotonin-induced accumbal dopamine release in an animal model of depression, Flinder sensitive line (FSL) rats. We previously found that FSL rats are characterized by absence of accumbal serotonin-dopamine interaction and antidepressant-induced restoration of this interaction directly correlated with improvement of their depressive-like behavior. In the current study, using in-vivo microdialysis, we found that: (a) $5 \mathrm{HT}_{2 \mathrm{C}}$ receptor blocking resulted in an increase in accumbal dopamine levels, which was significantly higher in FSL than in control rats; (b) $5 \mathrm{HT}_{3}$ receptor agonist administration resulted in elevation of accumbal dopamine levels, which was significantly lower in FSL than in control rats; (c) antidepressant treatment of FSL rats resulted in a decrease in $5 \mathrm{HT}_{2 \mathrm{C}}$ antagonist-induced and $5 \mathrm{HT}_{3}$ agonistinduced dopamine release; (d) the onset time for each antidepressant treatment for normalizing $5 \mathrm{HT}_{2 \mathrm{C}^{-}}$and $5 \mathrm{HT}_{3}$-mediated dopamine release was equal to that needed to improve depressive-like behavior of FSL rats. We concluded that: (a) the absence of accumbal serotonin-dopamine interaction in FSL rats can be explained by increased inhibitory-like activity of $5 \mathrm{HT}_{2 \mathrm{C}^{-}}$and decreased excitatory-like activity of $5 \mathrm{HT}_{3}$ receptors; (b) repetitive antidepressants decrease $5 \mathrm{HT}_{2 \mathrm{C}^{-}}$and increase $5 \mathrm{HT}_{3}$ receptor activity; (c) antidepressants with more rapid effect on $5 \mathrm{HT}_{2 \mathrm{C}}$ and $5 \mathrm{HT}_{3}$ receptor activity are characterized by a faster onset of behavioral effect of the treatment.

\section{Directional tuning of motor cortical neurons during} continuous and reaching movement

Drori R. Ben-Shaul Y. and Abeles M.

Hadasah Medical school and the Interdisciplinary Center for Neural Computation, Hebrew University of Jerusalem

Arm related neurons in the motor cortex are thought to represent the direction of arm movements. This is expressed by the tendency of neurons to elevate the firing rate mostly before and during a movement in a preferred direction (PD). This result was described when studying reaching movements. The purpose of this study was to check whether the same holds when continuous movements are performed

Two monkeys were trained to perform various types of movements in the horizontal plane holding a 2-joint manipulandum. Each monkey alternated between the center-out (CO) task that yielded straight trajectories in six equally-spaced directions, and another task (scribbling/free-tracing) that yielded curved, prolonged trajectories. During a scribbling trial the monkey moved the manipulandum freely in the horizontal plane until hitting an invisible position randomly chosen out of 19 positions that tiled the plane. Then another position was chosen and the monkey continued to move. During a free-tracing (FT) trial it followed one out of 40 predefined paths that were designed to yield prolonged trajectories with a uniform distribution of directions.

Neural activity was recorded from arm related areas in primary and pre motor cortices during task performance. Preferred directions of well isolated units were obtained from $\mathrm{CO}$ trajectories. Segmentation of continuous trajectories was carried out based on the corresponding velocity profiles so that relatively straight segments were extracted. For each cell, the PD from $\mathrm{CO}$ trajectories was compared to the PD obtained from continuous trajectories' segments. PDs obtained from FT segments were often similar to those obtained from $\mathrm{CO}$ trajectories. Unlike that, PDs obtained from scribbling segments tended to differ from those of $\mathrm{CO}$ trajectories. These results may therefore suggest that directional tuning of arm related neurons in the motor cortex tend to change when the context of movement is changed

Supported by grants from ISF and GIF

Extrasynaptic acetylcholine receptor repression deficit in dystrophin-deficient muscles of mutant (mdx) mice Durrant A.R., Salpeter M.M.", Anglister L.

Dept. Anatomy and Cell Biology, Hebrew Univ. Med. Sch. Jerusalem

The regulation and distribution of acetylcholine receptors (AChRs) are critical for proper function of the neuromuscular junction ( $\mathrm{nmj}$ ), and are influenced by specialized elements of muscle membrane, cytoskeleton and basal lamina and by nerve released compounds. Previously we showed that in dystrophin-deficient mutant $(m d x)$ mice the nmj's are structurally distorted and their AChR degradation rate is pathologically altered. The present study examines AChR distribulion to determine whether these abnormal muscles are able to repress their extrasynaptic AChRs and maintain the high concentration of junctional AChRs as in normal muscles. AChRs in sternomastoid muscles of $m d x$ and control adult mice ( 2 age groups; $m d x$ strain confirmed by elevated serum creatine kinase levels) were labeled in vivo with ${ }^{125} \mathrm{I}-\alpha$-bungarotoxin. Muscles were then excised; junctional and extrajunctional regions were separated and measured for radioactivity. AChR densities were calculated from the corrected radioactivity per muscle weight. In the mature group (43-52 wk old) extrajunctional AChR density in $m d x$ muscles was $9325 \pm 1652$ compared with $2051 \pm 732$ fmoles $/ g$ in the controls (mean $\pm \mathrm{SEM}, 4-5$ fold increase, $\mathrm{P}<0.01$ ). An extrajunctional AChR increase (3-4 fold) was also observed in $m d x$ muscles of the young adults (16-17 wk old). In both age groups the density of junctional AChRs in the $m d x$ muscles decreased to $65-70 \%$ of normal. These findings suggest that there is a deficit in the signaling pathway for extrasynaptic repression of AChRs in the $m d x$ muscles. Further studies will determine the relative contributions of nerve and muscle to this deficit in AChR regulation and help clarify whether this deficit is common to other neuromuscular diseases.

Supported by Israel Scl. Found. 685/01

M.M.Salpeter (Cornell Univ.) died on 24 October, 2000

DP-155, a phospholipid derivative of Indomethacin,

exhibits an improved therapeutic index in fever, analgesia and animal models Alzheimer's disease

Duvdevani R. ${ }^{1}$, Friedman J.E. ${ }^{1}$, Dvir E. ${ }^{1}$, Lee J.Y. ${ }^{2}$, Koh J.Y. ${ }^{2}$ Younis F. ${ }^{1}$, Raz S. ${ }^{1}$, Bello L. ${ }^{1}$, Polyak M. ${ }^{1}$, Shapiro I. ${ }^{1}$, Kozak A. ${ }^{1}$ and Angel I.

${ }^{1}$ D-Pharm Ltd. Rehovot. Israel; ${ }^{2} U$. of Ulsan, Seoul. Korea.

Although Indomethacin (Indo) is a widely used potent, anti-inflammatory drug, its use is limited by the high risk of unwanted gastrointestinal (GI) and renal side effects. To address this issue D-Pharm designed a novel, phospholipid derivative of Indo, DP-155. This is a comparative study of Indo and DP-155, at equimolar doses, assessing the in vivo efficacy, toxicity and pharmacokinetics (PK).

The antipyretic activity of Indo and DP-155 was studied in rats following induction of fever by SC injection of Brewer's Yeast. Analgesic efficacy was assessed using the Writhing and Formalin tests in mice. DP-155 showed a delayed onset of action as compared to Indo. DP-155 and Indo administered orally had similar analgesic and anti-pyretic efficacy starting at 2 and 3 hours, respectively.

The effect of oral administration for 3 days of DP-155 or Indo on A $\beta$-processing was tested on aged female $h A P P-T g 2576$ mice. Soluble $A B$ was extracted and analvzed using sandwich ELISA. Indo and DP-155 both significantly reduced the levels of A $442(29.7 \%$ and $23.6 \%$, respectively) without affecting A $\beta 40$ levels.

Total serum radioactivity measurements and HPLC analysis showed that DP-155 has a high bioavailability following oral administration in mice. The PK profile of Indo when administered as DP-155 orally to rats, was different from the one obtained following administration of Indo; demonstrating longer absorption time, broader $\mathrm{Cmax}$ and longer elimination half-life. PK studies of twice daily orally administered $\left[\mathrm{H}^{3}\right]-\mathrm{DP}-155$ to mice showed a longer retention of radioactivity in the brain post-dosing than for other organs. Following both single and multiple ( 3 davs) dosing. renal and GI toxicity parameters indicated that DP-155 is 5-10 fold safer than Indo. In conclusion, the data suggest DP- 155 as a superior derivative of Indo, potentially suitable for chronic treatment of Alzheimer's disease and peripheral inflammatory disorders. Support Contributed By: D-Pharm Ltd 
Retrograde transport of nerve growth factor (NGF) at the single molecule level

Echarte M.M. ${ }^{1}$, Pietrasanta L.I. ${ }^{1}$, Jovin T.M. ${ }^{2}$.

Centro de Microscopoas Avanzadas, FCEyN, Universidad de Buenos Aires; ${ }^{2}$ Dept. of Molecular Biology. Max Planck

Institute for Biophysical Chemistry

NGF is a peptide responsible for the regulation of survival, differentiation and maintenance of responsive neurons. It is released from the target tissue and interacts with receptors on the axon tips (Levi-Montalcini, 1987)

For neurotrophins to mediate survival effects, they must have the ability to transduce a series of complex signalling events from the nerve terminal plasma membrane to the nucleus situated in the cell body. NGF was the first neurotrophic factor shown to be retrogradely transported (Hendry et al., 1974)

To exert its effects. NGF interacts with two different receptors: TrkA and $\mathrm{p} 75$. Both receptors have been shown to be internalized and retrogradely transported in a signaling endosome, formed by the clathrin-mediated endocytic pathway. Based on their different kinetics of traffic and internalization, Bronfman et al. (2003) suggest that the signaling endosome containing the TrkA-NGF complex may be temporally and spatially different from that containing the p75-NGF complex

Our work is aimed to study the kinetics of the retrograde transport of NGF. To achieve our proposes, we plan to follow the internalization and traffic of NGF inside the PC12 cell, by using NGF coupled to biotin and quantum dots conjugated to streptavidin.

Quantum dots are nanometer-scale crystal of semiconductor material. Their high photostability and the fact that they are conjugated to streptavidin make them ideal probes for cellular imaging over long time. The possibility of visualizing NGF by conjugation with quantum dots, may allow us to track the motion of single molecules in real time in living celis. We have been able to visualize specific binding of quantum dots to differenciated PC12 cells membranes that have bcen preincubated with NGF-biotin. Current experiments are directed at visualizing the endocytic and internal trafficking stages.

In the context of a collaboration with Dr. Michael Fainzilber of the Weizmann Institute of Science.

Cyclin S: A new member of the cyclin family plays a role in long-term passive avoidance memory in the one-day-old chick

Edelheit $S$. and Meiri N.

Institute of Animal Science, Agriculture Research

Organization. The Volcani Center, Bet Dagan 50250

Memory is thought to be subserved by structural and functional alteration in synaptic connectivity. But although neuronal plasticity requires gene expression, the identity of the proteins involved is largely unknown. Using the chick one-day-old passive avoidance learning paradigm and differential display RNA fingerprinting we identified 13 candidate genes which are up-regulated in the intermediate medial hyperstriatum ventrale (IMHV), an area that has been correlated with the initial processing of memory formation. Among the genes induced in correlation with passive avoidance memory are N-CAM, HSP 90, EIF-2B epsilon, (phosphorylase kinase delta) (CALMI) and neurotensin/neuromedin $N$ precursor (NT/NMN). One of the induced genes is a new member of the cyclin family which was named cyclin $S$, with high homology to cyclin $L$ (ania-6a). Analysis of the expression pattern of this gene after training, revealed two time waves of induction: the first correlated with learning and initial memory process in the IMHV (a 2.5 -fold induction); the second, correlated with memory consolidation, first in the IMHV (a two fold induction, 24 hours after training), and then in the lobus paraolefactoris (LPO) (a 10 -fold induction $48 \mathrm{hr}$ after induction). There is a correlation between methylanthranilate (MeA) concentrations (The malaise substrate in the passive avoidance training procedure), the duration of memory, and the expression level of cyclin S. While training chicks on low concentration of MeA cause short-term memory and low expression level of cyclin S, high concentration of MeA induces long-term memory and high expression level of cyclin S in the IMHV. The role of cyclins in regulation of neuronal-plasticity-related gene expression was overlooked, and it might serve as a key step in long-term memory formation.
Effects of dexamethasone on neural differentiation and sprouting: implications for stress and depression Eick I. , Karry R., Klein E. and Ben-Shachar D. Laboratory of Psychobiology and Dept. of Psychiatry, Rambam Medical Center and B. Rappaport Faculty of Medicine, Technion

Substantial clinical and preclinical evidence implicate alterations of the stress hormone system in the etiology and development of depression. Depression is associated with an impairment of corticosteroid receptor function that gives rise to an excessive release of neurohormones, cortisol and CRH to which a number of signs and symptoms characteristic of depression can be attributed. In the past decade, research has focused on the involvement of long term intracellular processes in depression and stress leading to abnormal neuronal plasticity. To examine possible role for the synthetic glucocorticoid, dexamethasone (DEX) on neuronal morphology and plasticity human neuroblastoma SH-SY5Y cells were treated with $10^{-5}-10^{-7} \mathrm{M}$ of DEX for 24 and $48 \mathrm{hrs}$. SH-SY5Y cells treated with DEX showed a time and dose dependent decrease of cell number. Moreover, cells treated with $10^{-6} \mathrm{M}$ showed elongated granular rich cell body and increased number of neurites when compared with non treated cells. The decrease in cell number together with the morphological changes suggests a role for glucocorticoids in cell differentiation. These changes were accompanied by a dose and time dependent increase in CREB, the transcription factor involved in plasticity and implicated in stress and depression. However, the expression level of two neurite-outgrowth promoting genes, CAM-L1 and laminin was decreased. Interestingly, in a previous study we have shown that the expression of both $C A M-L 1$ and laminin. was increased while that of CREB was unchanged by norepinephrine. The diverse effect of glucocorticoid and of norepinephrine, as well as its relevance to their role in depression will be discussed.

\section{Reconsolidation blockade in the medaka fish may disrupt} memory retrieval rather than storage

Eisenberg $M$. and Dudai $Y$.

Dept. of Neurobiology, Weizmann Institute of Science

All items in long-term memory undergo a period of stabilization, termed consolidation, immediately after the acquisition event. The textbook account is that for each item in memory consolidation starts and ends just once. Recent evidence casts doubt on this assumption. We have recently demonstrated using both conditioned taste aversion in the rat and fear conditioning in medaka fish, that when conflicting long-term associations are activated in retrieval, that association that retains or gains control over behavior afterwards, becomes susceptible anew to agents that block consolidation i.e. reconsolidates (Eisenberg et al. Science $2003,301,1102$ ). However, whereas consolidation blockade is assumed to lead to memory storage deficit, it is yet unknown whether reconsolidation blockade leads also to storage deficit, or, alternatively, to retricval deficit, which means that despite the apparent amnesia the item is still retained memory though in a latent mode. We report evidence that in fear conditioning in the medaka fish, the memory blocked by the reconsolidation blockers does not recover spontaneously (within 7 days), but can still be reinstated by presentation of the unconditioned stimulus (mild electric shock) in the absence of conditioned stimulus (light). These data together with our recent findings in conditioned taste aversion in the rat (see Kobilo and Dudai, these proceedings), suggest that blockade of post-activation consolidation of long-term memories disrupts retrieval, and raises the possibility that reconsolidation is required to reupdate retrieval access to the activated memory.

Supported by the Israel Science Foundation, the Volkswagen

Stiffiung and the Human frontiers science Program.

Binocular rivalry mechanisms in adults and children Eisenberg M. Hochstein S. and Kovacs I.

Hebrew University of Jerusalem

Binocular rivalry occurs when the brain cannot fuse the images seen by the two eyes because they are two completely different pictures or differ in one or more features such as: color, orientation, size, motion velocity or luminance. After an initial $150-200 \mathrm{~ms}$ attempting to fuse the images, (where perception is of the two superimposed), perception alternates between two percepts, usually corresponding to the two eye's views, respectively. The debate is still open regarding the locus of binocular rivalry, i.e. what is the neural site of binocular alternations? where is the switch located in the brain? Based on recent research it seems that a number of different mechanisms are involved, found at low and high cortical levels. The consensus is that alternations occur at distributed sites, following the hierarchy of cortical processing: While 
local stimuli induce solutions (and alternations) at early cortical sites, complex stimulus configurations induce higher-level perceptual interpretations. If indeed binocular rivalry depends on distributed cortical sites, its development should correspond to their maturational course. To address this issue, we asked: Do children observe binocular rivalry? What is the temporal characteristic of rivalry alternations in young children? We presented preschool children with rivalrous anaglyph images of orthogonal gratings. The children were informed that they would see a horizontal and a vertical grating in alternation, and were asked to press a button whenever the grating changed its orientation. Most of the children in the 5-6 year-old group reported alternations. Some saw no rivalry at all (no alternations); some had very fast alternations and some very slow and some reported piecemeal rivalry. The shape of the frequency histogram of dominance durations resembles that of the adults and can be fit with a gamma function. Comparing the two histograms, one notes that children switch faster than adults. The faster rivalry alternation found in children could reflect actually faster switching, or could be caused by the children experiencing more piecemeal rivalry and pressing as if local fluctuations were full alternations. Either hypothesis could be explained by an immaturity of part of the visual cortex that is involved in rivalry. Since VI develops early, perhaps young children experience rivalry that corresponds to a V1 mechanism - i.e. local rivalry or patchwork. The integration of parts necessary for perception of the full picture and for "holding the percept together" happens "higher" in temporal cortex, which develops later, and therefore is only present in older children and adults. Supported by the Israel Science Foundation, grant \# 8009

\section{Proteomics approach to study the rule of ERKI/II in} learning and plasticity

Elkobi A. ${ }^{1}{ }_{2}$ Kaphzan $\mathrm{H}^{1}{ }^{1}$, Admon A. ${ }^{2}$, Ziv T. ${ }^{2}$ and Rosenblum $\mathrm{K}$

${ }^{I}$ University of Haifa, Center for Brain \& Behavior; ${ }^{2}$ Technion, protein center, faculty of biology, Haifa.

We are interested in the role of the extracellular regulated kinase I/II (ERKI/II) in the formation of long-term memory and plasticity. ERKI/II is involved in both early and late phases LTP (Rosenblum et al. $J$. Neuroscience (2002)). In addition, ERKI/II activity is correlated and necessary for the formation of long-term memories. In order to understand the molecular details of ERKI/II role in taste memory formation we aim to identify ERKI/II substrates in the taste cortex that are involved in taste memory formation (novel taste learning). We identify ERKI/II substrates using antibody that recognizes phosphorylated-Threonine only when followed by the amino acid Proline (the favorite phosphorylated site by MAP kinase) together with proteomics (two dimensional electrophoresis, mass spectrometry, and bioinformatics). We identified several proteins phosphorylated on Thr-Pro in synaptosomal fraction as well as total homogenates made from the insular cortex. We differ between ERKI/II substrates and other potential kinases using the MEK inhibitor U0126 on brain slices. Following the results obtained with the proteomics approach we use regular Western blot analysis to confirm the protein list. We identified known ERKI/II substrates (e.g. synapsin1) and novel proteins that may explain the role of ERK K $/ / / \mathrm{I}$ in plasticity and learning.

\section{Mapping chromosome 18p11 to normal personality}

Elhadad (Rosolio) N.', Dina C. ${ }^{2}$, Zohar A.H ${ }_{4}^{3}$, Osher Y.Error! Reference source not found. ${ }^{1}$, Gritsenko $I^{4}{ }^{4}$

Bachner-Melman R. ${ }^{4}$ Agam $G^{1}$, Birk O. ${ }^{1}$, Benjamin J.', Belmaker $\mathrm{RH}^{1}$ and Ebstein RP

'Faculty of Health Sciences, Ben-Gurion University, Beer-Sheva, Israel; ${ }^{2}$ Genetique Statistique - Institut de Biologie de Lille, UPRES A 8090 Lille, France; ${ }^{3}$ The Ruppin Institute of Higher Education Emek Hefer, Israel; ${ }^{4}$ Dept. of Research, S. Herzog Memorial Hospital \& Psychology Dept. (Scheinfeld Center), Hebrew University, Israel;

Human beings differ widely in the style, temper, vigor or emotional expression accompanying their behavior. Use of self-report questionnaires have characterized several personality factors, stable over time and across cultures. Personality traits were suggested to be endophenotypes for major mental illnesses. Therefore, chromosomal regions (such as $8 p$ and $18 p$ ) linked to mental illnesses are also expected to be common loci that predispose emotional lability that later provides a causal link to psychosis. A chromosomal area of interest for harboring Quantitative Trait Loci (QTLs) contributing to normal personality traits is chromosome $18 \mathrm{p}$ since it both contains a locus for bipolar disorder and is linked to schizophrenia. We examined a group of 7 closely arranged $(\sim 10-15 \mathrm{cM})$ microsatellite markers in the $18 \mathrm{p} 11$ region in healthy 182 sibling pairs with two parents, 14 sibling pairs with one parent and 376 sibling pairs with no parents. Subjects were both Ashkenazi and non-Ashkenazi Jews who filled out the Tridimensional Personality Questionnaire (TPQ) questionnaire. Genomic DNA was extracted from periphera blood or mouth washes using phenol-chloroform. PCR products obtained using primer pairs for the microsatellite markers were separated on an ABI Prism 310 Genetic Analyzer.

Association of each individual marker within these families to the four TPQ personality dimensions was examined using th Quantitative Transmission Disequilibrium Tests (QTDT) statistical package. Overall global $p$ value was significant only for the D18s1150 marker ( $p<0.05$ following Bonferroni between these microsatellite markers and TPQ personality traits, weak evidence ( Lod score 1 ) for linkage for Novelty Seeking was observed.

p53 inhibitor reduces PrPsc acumulation in-vitro, but has no effect in-vivo

Engeistein $\mathrm{R}^{1}$ Shaked M.G. ${ }^{1}$, Ovadia H. ${ }^{1}$, Greig N.H. ${ }^{2}$, Grigoriadis N ${ }^{3}$ and Gabizon $\mathrm{R}^{3}$.

Dept. of Neurology; The Agnes Ginges Center for Human Neurogenetics, Hadassah University Hospital, Jerusalem Israel ${ }^{;}$Laboratory of Neuroscience, National Institute on Aging Gerontology Reaserch Center, Baltimore, USA; ${ }^{3}$ Dept of Neurology AHEPA, Aristotale University Hospital,

Thessalonoki, Greece

The only identified component of the prion infectious agent (causing scrapie and other neurodegenerative diseases) is the prion protein $\left(\mathrm{PrP}^{\mathrm{sc}}\right)$, the abnormal isoform of the normal protein $\operatorname{Pr}^{\mathrm{c}}$. As for today, the mechanism of pathogenesis of prion diseases have not been elucidated. Several investigators have proposed that apoptosis plays a role in prion disease pathogenesis. Apoptosis, defined as programmed cell death, was shown to be mediated by several factors and proteins, and is easily characterized by the ordered damage in the DNA. In several diseases, inhibition of apoptosis was shown to affect disease course.

We show here that Pifitrin $\alpha$ (PFT), a newly synthesized p53 inhibitor can inhibit the accumulation of $\operatorname{Pr} \mathrm{P}^{\mathrm{sc}}$ in ScN2a cells, a neuroblastoma cell line

permanently infected with prions and expressing $\mathrm{PrP}^{\mathrm{sc}}$ Following these encouraging in-vitro results, we proceeded to test the effect of PFT in-vivo. Hamsters infected with scrapie prions were subsequently treated with PFT or with vehicle only were observed for symptoms of disease. Incubation time from inoculation to disease was established for all gropus. Our results indicate that PFT did inhibit apoptosis in the treated hamsters, as seen by measuring caspase-3 expression by both western bloting and immunohistochemistry. Howerer, such inhibition had no affect on disease course, incubation time or $\operatorname{PrP}^{\mathrm{P}^{\mathrm{S}}}$ and accumulation of $\operatorname{Pr}^{\mathrm{Sc}}$ in the infected hamstres brains. We conclude that although apoptsis of neurons seems to play some role in the disease, reduction of apoptosis does not aleviate the symptoms or the severity of the disease.

High frequency local field potentials in the striatum of the halothane-anaesthetized rat

Engler G. ${ }^{1}$, Moll C.K.E. ${ }^{1}$, Bergman $\mathrm{H}^{2}{ }^{2}$ and Engel A.K. ${ }^{1}$ Institute of Neurophysiology and Pathophysiology, University Hospital Hamburg-Eppendorf, Hamburg, Germany: ${ }^{2}$ Faculty of Medicine. The Hebrew University, Jerusalem. Israel

Recent animal studies and human neurophysiological investigations have placed special emphasis on both the physiological and pathophysiological role of synchronization and oscillations within the basal ganglia circuitry.

In this context, high-frequency local field potential (LFP) oscillations in the upper gamma-frequency band $(40-80 \mathrm{~Hz})$ have been recorded from the subthalamic nucleus (STN) in parkinsonian patients treated with levodopa (Cassidy. et al 2002) and the STN of the healthy alert rat (Brown et al., 2002). Together with electrocorticograms from both hemispheres, we recorded single-and multiunit activity as well as local field potentials (LFPs) bilaterally using eight microelectrodes placed in the striatum of the healthy halothane-anaesthetized rat. Here we report on the analysis of the local fields: Field-Potential-Analysis shows that (i) LFPs recorded from the striatum of the anaesthetized rat have a spectral peak in the upper gamma-band (range $55-75 \mathrm{~Hz}$ ); (ii) pronounced coherence in the high gamma-band occurs between cortex and striatum while beta-band-coherence $(15-30 \mathrm{~Hz})$ is low between cortex and striatum but high within the striatum; (iii) strong low-frequency-coherence $(<5 \mathrm{~Hz})$ occurs within and across the hemispheres. Our findings suggest that high-frequent oscillatory activity within the basal ganglia is physiological and may hold a certain function in information processing within the different cortico-basal ganglia-thalamic loops since it is encountered in different subcortical structures. 
Furthermore it appears to be conserved across different species and different vigilance states. Further studies are needed to investigate the origin of this high-frequent subthreshold activity and its relationship to other brain signals, eg its correlation with neuronal firing.

Cassidy $M$ et al., Brain 125 (2002): 1235-46; Brown $P$ et al., Exp Neurol 177 (2002):581-85.

Supported by BMBF-MOS, Project-No. 01GA030I

\section{Selective capturing of transported vesicles by} microtubule-based traps formed by axotomy Erez H. and Spira M.E.Error! Bookmark not defined. Dept. of Neurobiology. Life Sciences Institute. The Hebrew University of Jerusalem.

Supply, targeting and fusion of new membrane with the growth cone (GC) plasma membrane is necessary to assure neuronal outgrowth.

Using cultured Aplysia neurons and on line confocal imaging of MTs, anterogradlly and retrogradlly transported vesicles we found that axotomy leads to the formation of specialized "vesicle traps" that localize the supply of vesicles to the cut ends of the proximal and distal axonal segments.

In the proximal axonal segment, the cut end forms two types of traps. (A) A trap in which all the MTs plus ends point to the center of the growth cone. This MTs configuration leads to the accumulation of vesicles linked to molecular motors that move towards the MTs $(+)$ ends. With time, new MTs polymerize centrifugally from the center of the $\mathrm{GC}$, and the trapped vesicles translocate along the MTs towards the $(+)$ ends to fuse with the plasma membrane and support the growth process. (B) The second trap is a (-) end trap, located few micrometers distally to the $(+)$ end trap. This trap localizes vesicles that are mobilized by molecular motors that move towards the (-) ends of the MTs. The trapped vesicles at this location are gradually transported retrogradly while a GC's lamellipodium extends from the $(+)$ end trap.

In the distal axonal segment, only a (-) end trap is formed. Accordingly retrogradlly transported retrieved plasma membrane, labeled by the fluid phase pinocytotic marker (sulforhodamin 101) is localized to that (-) end trap. With time new MTs polymerize from the center of the $(-)$ trap. Nevertheless, the sulforhodamin 101 labeled vesicles remain within the trap and the development of a GC structure ceases. Supported by the ISF No. 556/01 and BSF No. 2000354

ErbB-4 receptor tyrosine kinase and NRG functions in neuronal survival and differentiation

Erlich S. ${ }^{1}$, Goldshmit Y. ${ }^{1}$, Shohami E. ${ }^{2}$ and Pinkas-Kramarski $\mathrm{R}^{\mathrm{I}}$

${ }^{i}$ Dept. of Neurobiochemistry. Tel-Aviv University, Tel Aviv; ${ }^{2}$ Dept. of Pharmacology, the Hebrew University, Jerusalem

The ErbB-4 receptor tyrosine kinase and its ligand neuregulin (NRG) are widely expressed in the nervous systems. To investigate their possible role in neurodegeneration we used the closed head injury model. We demonstrate that levels of ErbB-4 are dramatically increased at the site of injury. The staining patterns indicate that activated microglia/macrophages and neurons but not astrocytes, constitute the major population of cells that highly express the receptor at the injury site. Confocal microscopy analysis suggests that the high levels of ErbB-4 protein in activated microglia/macrophages is probably due to phagocytosis of neuronal cells. These findings indicate that ErbB-4 receptors may play a role in brain responses to head trauma. After brain insult or trauma, astrocytes become activated and attempt to restore the brain's fine tuned microenvironment. Intracerebrally administration of NRG was used to investigate the possible role of $N R G$ in-vivo. We demonstrate that direct injection of NRG into the brain increases GFAP expression and alters astrocytes morphology. This significant effect of NRG on astrocytes activation persisted for at least 7 days following injection. These findings show for the first time that in-vivo NRG play a role in astrocytc activation. In addition, our study demonstrates that following closed head injury NRG levels increase in astrocytes at the site of injury. These findings indicate that NRG might serve as a potent activator of astrocytes and that it might be part of the mechanism of glial activation observed following CNS injuries and trauma in an autocrine manner.

To examine the possible role of ErbB-4 in neurons we have used the PC12 cell. Expression of ErbB-4 in PC12 cells demonstrated that NRG induced signals and neurite outgrowth, which were indistinguishable of those mediated by NGF. In PC 12-ErbB-4 cells, NRG induced an initial weak mitogenic signal and subsequently neurite outgrowth as well as rescued from apoptotic cell death. Our results demonstrate that neurite outgrowh induced by ErbB-4 in PC12 cells, requires MAPK and $P K C$ signaling networks and $N R G$ induced survival from apoptotic cell death requires PI3K signaling.
Modified cytoarchitecture in glutamatergic neurons under peripheral site inhibition of acetylcholinesterase Farchi N. ${ }^{1,2}$, Dong $\mathrm{H}^{3,4}$, Eilon K. ${ }^{2}$, Hochner B. ${ }^{1}, \mathrm{Lu}$ W-Y ${ }^{3,4}$ and Soreg $\mathrm{H}^{2}$.

Dept. of Neurobiology and ${ }^{2}$ Biological Chemistry, The
Hebrew University of Jenusalem, Israel; Dept. of Anaesthesia and ${ }^{4}$ Physiology, University of Toronto, Canada.

Acetylcholinesterase (AChE) inhibitors serve as Alzheimer's disease drugs, but their mechanism of action is yet incompletely understood and their effects are transient. AChE contributes to neuronal differentiation, cell adhesion and synaptogenesis through yet unknown cellular and molecular processes unrelated to its catalytic properties. Here, we report that ligands selective for the peripheral site of AChE (e.g. BW284c51), but not inhibitors selective for its catalytic site (e.g. physostigmine), trigger in cultured hippocampal neurons impaired glutamatergic currents associated with membrane. In BW284c51-treated cells, transcriptional and alternative splicing responses lead to somatic redistribution of alternative splicing responses lead to somatic redistribution of stress-induced AChE-R mRNA translocated into dendrites. Co-treatment with an antisense agent inducing AChE-R mRNA destruction abolished this intricate feedback response to BW284c51 and rescued glutamate currents. RACK1, a perikaryal PKCaII cargo protein which interacts with AChE-R, decreased in neuronal processes under BW284c51 with significantly lower accumulation near the cell membrane. Moreover, Synaptophysin, Neuroligin and $\beta$-Actin labeling greatly increased in soma while decreasing in neuronal processes, contrasts with AChE or MAP2 over-expression. Our findings attribute to neuronal AChE mRNA redistribution causal role in the poor surface expression of glutamate receptors, associated with selectively altered neuritic protein transport under peripheral inhibition of $\mathrm{AChE}$. These results suggest adverse long-term consequences to the administration of peripheral $\mathrm{AChE}$ inhibitors.

Spectral and temporal response patterns of auditory cortex neurons under barbiturate anaesthesia

Farkas D., Bar-Yosef O., Ulanovsky N., Las-Ahdut L. and Nelken I.

Dept. of physiology, Hebrew University - Hadassah Medical School, and the Interdisciplinary Center for Neural

Computation, Hebrew University.

Anaesthesia is known to strongly affect responses in auditory cortex. Most studies of auditory cortex neurons have been done under barbiturate anaesthesia. Under these conditions, most neurons have mostly onset responses and unimodal, sharply tuned frequency response areas.

We recorded tone responses of over 1300 neurons from the primary auditory cortex (AI) of 26 cats, anesthetized by a mixture of $\mathrm{O}_{2} / \mathrm{N}_{2} \mathrm{O}(30 \% / 70 \%)$ with halothane $(0.2-1.5 \%$, as required). This protocol enabled us to keep the cat well-anesthetized under a lighter regime than can be achieved with barbiturates. Tone bursts (115 ms, with $10 \mathrm{~ms}$ linear onset and offset ramps) were presented at $8-11$ equally spaced levels (5-10 dB resolution) and 45 frequencies equally spaced along a logarithmic frequency axis $(6-12 / \mathrm{Oct})$ over a range covering the fill frequency response of the neuron.

We observed a much richer array of tuning curve shapes than are found under barbiturate anaesthesia, including multi-lobed FRAs $(30 \%)$ which were characterised by low compactness (the total area of the FRA divided by the perimeter of the tuning curve), wide-band neurons that respond over a large frequency range $(38 \%$, with $\mathrm{Q} 30<0.5)$ and a large number of neurons with very low minimal thresholds $(35 \%$, with threshold <20dB).

We used the mutual information (MI) to quantify the We used the mutual information (MI) to quantify the neurons had informative responses extending beyond onset. A substantial number of neurons $(25 \%)$ had informative activity extending beyond stimulus offset for at least $100 \mathrm{~ms}$. The different parameters of the response (compactness, $\mathbf{0 3 0}$, threshold, MI, spike count) showed very low correlations between them. The most pronounced correlations were found between the mutual information and compactness $(r=0.3$. df $=1334, p<0.05)$ and between the mutual information and the spike count $(r=0.6 \mathrm{df}=1334, \mathrm{p}<0.05)$ The large variability of response properties and the low correlations between the different parameters suggest low redundancy between the neurons. Moreover, the informative responses long after the stimulus offset demonstrate the presence of short-term memory in primary auditory cortex. Thus, using halothane anaesthesia is a reasonable compromise for research in auditory cortex, since tonal responses are rich and varied, and they keep many of the neuronal properties described in the awake animal. 
Presynaptic membrane of inhibitory crayfish axon terminals is stained by antibodies raised against mammalian GABA $\boldsymbol{A}_{\mathbf{A}}$ receptor subunits $\boldsymbol{\beta}_{3}$ and $\boldsymbol{\beta}_{2 / 3}$

IOtto Loewi Minerva Center for Cellular and Molecular Neurobiology, Hebrew University, Jerusalem, Israel Institute of Pharmacology and Toxicology, University of Zurich, Winterthurerstrasse, Zurich, Switzerland

The opener muscle of the dactyl of the walking leg of crayfish is innervated by one excitatory axon releasing glutamate and one inhibitory axon releasing GABA. Functional $\mathrm{GABA}_{\mathrm{A}}$ receptors are present postsynaptically on the muscle, and presynaptically on terminals and release boutons of the excitatory axon, whereas presynaptic GABA autoreceptors have not been reported on terminals or release boutons of the inhibitory axon. Using antibodies raised against mammalian $\mathrm{GABA}_{\mathrm{A}}$ receptor subunits $\alpha_{3}$ and $\beta_{2 / 3}$, we obtained highly specific staining of the presynaptic membrane of the inhibitory bouton and of the postsynaptic membrane of the muscle. Using pre- and post-embedding techniques, staining was localized to only presynaptic and postsynaptic membranes of synaptic active zones. We also found near (up to $100 \mathrm{~nm}$ ) to the active zones extrasynaptic receptor subunit immunoreactivity. Staining with antibodies for the $\alpha_{3}$ and $\beta_{2 / 3}$ subunits showed colocalization of particles of the two subunits. We suggest that presynaptic inhibitory boutons of the crayfish possess

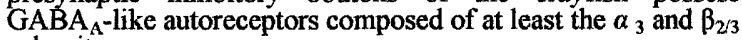
subunits.

Mitochondria and vesicles as calcium regulators

Fęndyur A. ${ }^{1}$, Raveh A. ${ }^{1}$, Blank P. ${ }^{2}$, Kaiserman $~^{1}{ }^{1}$, Coorssen

$\mathrm{J}{ }^{3}$, Zimmerberg $\mathrm{J}^{2}$ and Rahamimoff $\mathrm{R}$.

${ }^{I}$ Dept. of Physiology. \& Bernard Katz Minerva Centre,

Hebrew University, Jerusalem, Israel; ${ }^{2} N I H$, Bethesda, MD,

USA ${ }^{3}$ U. Calgary, Calgary, Canada.

Intracellular calcium ions $\left(\left[\mathrm{Ca}^{++}\right]_{\text {in }}\right)$ play a key role in a wide variety of neuronal functions. They are regulated by mitochondria vesicles and the endoplasmic reticulum. We studied mitochondrial $\mathrm{Ca}^{++}$dynamics in neuronal processes of rat hippocampal cultures and vesicle calcium dynamics in secretory vesicles from sea urchin eggs. Mitochondria. Cultured hippocampal neurons (4-9 days old) of neonatal Sabra rats were used to study mitochondrial calcium dynamics. Using mitochondrial and calcium-specific fluorescent dyes (Fluo-4 and Mitotracker) we double stained hippocampal neurons and simultaneously sampled both mitochondrial location and $\mathrm{Ca}^{++}$dynamics. Three main observations were made: there is calcium self-talk within the mitochondrion there is calcium cross-talk between the mitochondrion and the cytosol and there is calcium cross-talk between mitochondria. The secretory vesicles in the nervous system are too small for calcium imaging. However, the secretory vesicles in sea urchin eggs are large $(\sim 1.5 \mu \mathrm{m}$ in diameter), allowing quantitatively meaningful confocal microscopy. Calcium indicators with different affinities were used to study calcium dynamics in different calcium concentrations, while lypophilic and $\mathrm{pH}$ indicators were used to eliminate artifacts due to vesicles movements or $\mathrm{pH}$ changes, respectively. The secretory vesicles behave as single units with respect to changes in their $\mathrm{Ca}^{++}$ fluctuations. While their total $\left[\mathrm{Ca}^{++}\right]$is $\sim 100 \mathrm{mM}$, using different fluorescent indicators, the free $\left[\mathrm{Ca}^{++}\right]$of the secretory vesicles were estimated to be in the micromolar range

In both organelles calcium waves were observed. In $6 \%$ of the mitochondria and in $\sim 2 / 3$ of the vesicles spontaneous poin waves were detected. These results and the distribution of these two organelles in the presynaptic region reflect their importance in modulating the presynaptic fusion process and other $\mathrm{Ca}^{++}$dependent intracellular functions.

Supported by grants from: BSF, ISF and Bernard Katz

Minerva Centre.

Early weaning and isolation interact to increase anxiety and social interaction in adult rats

Ferdman N. ${ }^{1}$, Braun $\mathrm{K}^{2}$ and Leshem $\mathrm{M}^{\mathrm{t}}$

Psychology Dept. and Brain \& Behavior Center,

The University of Haifa; Israel: "Institute for Biology.

Otto-von-Guericke-University. Magdeburg Germany

We compared the influence of typical weaning, at 21-days-of-age, with later weaning, at 30-days-of-age, on open field behavior and a social interaction test in adulthood. After weaning, pups were reared in individual cages or in groups, to distinguish the effects of weaning from social isolation. Wistar pups born in the lab were raised with their dam until 21 or 30 days of age. These early and late weaning pups were further subdivided into rats reared in individual cages ('isolation') or in group cages. All rats were tested in adulthood (14 weeks) in the open field and the social-interaction test ( 3 min each test). In the open field, early weaning increased peripheral and total number of crossings. In the social interaction test, isolation increased sniffing of the strange rat, and in the early weaned rats it increased social grooming of the strange rat. Early weaned and isolated rats spent more time in the periphery than group-reared late-weaned rats, that were also more active especially in the center of the open field. Our findings suggest that early weaning and isolation increase anxiety in a novel situation in adulthood, and increase social interaction with a strange rat. The anxiogenic effects of isolation appear irreversible, but the social effects of isolation are mitigated by late weaning. Thus 9 more days with the dam (late weaning) apparently protect against the influence of 90 days of subsequent isolation on social responses in adult rats. In turn, this suggests that weaning is a critical formative period for adult social behavior.

\section{Activity dependent cell survival in dissociated neuronal} culture

Fishbein I., Maggio N. and Segal M.

Dept. of Neurobiology, Weizmann Institute, Rehovot 76100

It is generally believed that spontaneous bioelectrical activity (SBA) within the nervous system is required for the development of the cerebral cortex. SBA has also been shown to be a key factor in establishing neuronal network and synaptic connectivity. In dissociated tissue culture, chronic suppression of SBA can increase the rate of cortical cell death (Ramarkers. and Boer Eur. J. Neurosci, 3: 140). To further characterize the importance of SBA for neuronal development nd mantainance, we used dissociated culture from posterior corticai areas. To inhibit SBA, we treated postnatal day 3 cultured neurons with $0.1 \mathrm{uM}$ tetrodotoxin (TTX). We monitored cell survival rate by staining them with anti NeuN, a specific neuronal transcription factor. Massive cell death was detected after 10 days of chronic TTX treatment. Interestingly, inhibiting SBA by blocking AMPA and NMDA receptors did not have the same effect. Furthermore, TTX treatment combined with NMDA and AMPA receptor antagonists was found to partially prevent TTX-induced cell death. We also found that the neurotrophin BDNF rescued neurons from TTX-induced cell death. These results suggest a key role for the presynaptic activity and its possible mediation by endogenous BDNF release.

Role of somatostatin and corticotropin releasing factor in regulation of prostaglandin synthesis in glial cells

Fleisher-Berkovich S., Naama D.

Dept of Clinical Pharmacology, Faculty of Health Science,

Ben Gurion University, Beer-Sheva

Considerable evidence shows involvement of chronic inflammation in the pathogenesis of Alzheimer's disease (AD). This chronic inflammatory response is characterized, among other things, by reactive gliosis in which glial cells are activated and proliferate. Activated glial cells are a major source of two important mediators of inflammation, namely prostaglandins ( $\mathrm{PGs}$ ) and nitric oxide (NO). The aim of the present study was to investigate the role of neuropeptides such as somatostatin and corticotropin releasing factor in the regulation of basal and lipopolysaccharide (LPS)-induced PG and NO production in glial cells. Our results show that SS $\left(10^{-9}-10^{-7} \mathrm{M}\right)$ inhibited basal PG synthesis by $64 \%-68 \%$ and NO production by $46 \%-78 \%$. LPS increased PG and NO synthesis in rat glial cells in a dose and time dependent manner. SS $\left(10^{-50} \mathrm{M}-10^{-9} \mathrm{M}\right)$ inhibited LPS-induced PG synthesis by $40 \%-50 \%$ and NO production by $26 \%-30 \%$. Interestingly, phosphoramidon, a neutral endopeptidase (NEP) inhibitor, enhanced prostaglandin $\mathrm{E}_{2}$ synthesis in glial cells several fold CRF significantly increased prostaglandin synthesis but abrogated phosphoramidon-enhanced prostaglandin production. It is tempting to speculate that if brain $P G$ and NO synthesis that is induced by inflammatory agents like LPS is significantly regulated by neuropeptides, then specific preventive and therapeutic modalities may be devised to intervene with these mechanisms.

\section{5-lipoxygenase and cytochrome P-450 pathways of} arachidonate metabolism contribute to endogenous cryogenesis

$\frac{\text { Fraifeld V }}{{ }_{\text {Wolfson }}{ }^{1}}{ }^{1}$ Paul L. ${ }^{1}$, Kaplanski $J^{1}{ }^{1}$, Asraf $\mathrm{H}^{1}{ }^{1}$, Sagi O. ${ }^{1}$, Wolfson M$^{1}{ }^{1}$, Kozak W. ${ }^{2}$, Kluger M.J. ${ }^{2}$, Tchaikovskaya T. ${ }^{3}$ and Listowsky I.

${ }^{I}$ Ben Gurion University, Beer-Sheva, Israel; ${ }^{2}$ MCG, Augusta, GA: ${ }^{3}$ AECOM, Bronx, NY

Studies on the involvement of the arachidonic acid (AA) cascade in thermoregulation have primarily focused on breakdown products of the COX pathway, mainly PGE. This eicosanoid is considered to be the final central mediator of fever. Recent data obtained in our laboratories, suggest a thermoregulatory role for two other pathways of AA, 5-lipoxygenase (5-LO) and cytochrome $\mathrm{P}-450$ monooxygenase (epoxygenase). 
Herein we report that the central, most likely, hypothalamic leukotrienes (LTs), 5-LO-derived eicosanoids, and epoxygenase-derived eicosanoids (EETs) contribute to the process of endogenous antipyresis or cryogenesis. Several lines of cvidence are in support of this hypothesis: (1) LPS-induced hypothermia in mice is preceded and accompanied by an elevation in hypothalamic cysteinyl-LT production; (2) the inhibitor of LT synthesis MK-886 injected into the lateral ventricle or ip, suppresses both of these processes; MK-886 also prevents the decrease of body temperature $\left(T_{b}\right)$ during the light period of the day; (3) icv injections of $\mathrm{LTC}_{4}$ at nanomolar doses cause a dose-dependent decrease of daytime $T_{b}$ and reduce the nighttime rise of $T_{b} ;$ (4) icv injections of EETs, as well as inducers of P-450, suppress enhance the fever response in rats; (5) 11,12-EET inhibits the activity of COX-2; (6) more pronounced hypothermic $T_{b}$ response to LPS in older mice correlates with the age-related increase in the level of brain 5-LO; (7) the expression of neuronal 5-LO mRNA is down-regulated in mGSTM5 KO mice compared to the wild-type animals. Accordingly, mGSTM5 KO mice develop more pronounced fever in response to low doses of LPS and lesser hypothermia after high doses. The results obtained highlight the role of 5-LO and cytochrome P-450 pathways in endogenous cryogenesis. Supported by grants from the BSF, Jerusalem, Israel and from the Atran Foundation, Albert Einstein College of Medicine, Bronx, NY

Inability to suckle after blockade of cannabinoid receptors: A novel animal model for "failure to thrive" in newborns Fride E. ${ }^{1,2}$, Ezra D. and Blau H.

${ }^{7}$ Dept. of Behavioral Sci. and ${ }^{2}$ Molecular Biology, College of Judea and Samaria, Ariel; ${ }^{3}$ Pulmonary Unit, Schneider

Children's Medical Center, Petach Tikva

Children with 'Non-organic failure to thrive' (NOFTT), have an abnormally low weight and/or height for age without identifiable organic etiology, except for a putative oral-motor dysfunction. An unidentified biological vulnerability has been invoked to explain NOFTT. We have reported that the newly discovered physiological system comprising endogenous cannabinoids and their receptors (the "endocannabinoid" system), play a critical role in milk ingestion and survival of the newborn. Thus specific blockade of the cannabinoid (CB1) receptors in one-day old mouse pups prevented milk intake and resulted in death within days after birth. Similar deficiencies were found in (untreated) $\mathrm{CBl}{ }^{\square /-}$ knockout pups

In the present series of experiments, we investigated the hypothesis that a deficient endocannabinoid system underlies NOFTT. Therefore we directly studied suckling behavior and behavioral/physiological mechanisms in mouse pups, which had been treated with the $\mathrm{CB} 1$ receptor antagonist (SR141716A, 'SR1') within $24 \mathrm{~h}$ after birth.

Suckling with an anesthetized dam was studied on days 2, 4,6 and 9. Weight gain, ultrasonic vocalizations (USVs) and axillary temperature were measured daily, in SR1-treated Sabra, ICR, and $\mathrm{CB}^{\mathrm{D} /}$ - receptor knockout pups.

Results: 1. 80-100\% mortality was observed within days after SR 1 injection 2 . Suckling was completely suppressed in day 2 old antagonist-treated pups and partially in CB1 knockouts. 3. Persistent hypothermia and USV suppression were observed in SR1-treated pups and in neonatal $\mathrm{CB1}^{-1-}$ knockouts 4. Hypothermia and reduced USVs were recorded in the non-surviving SR1 pups already before injection

Conclusions: a. $\mathrm{CBl}$ receptor blockade interferes with the ability of the newborn to attract maternal care and $\mathbf{b}$. induces oral-motor deficiency, thus preventing suckling $c$. These symptoms are compatible with the symptomatology of NOFTT d. A deficient endocannabinoid system may underlie NOFTT. Supported by Danone Research Institute in Israel

Lasting neuronal dysfunction following cortical blood-brain-barrier disruption

Friedman A. ${ }^{1,3}$, Seiffert E. ${ }^{1}$, Dreier J.P. ${ }^{2}$, and Heinemann U. ${ }^{1}$ Thysiology and ${ }^{2}$ Experimental Neurology, Charit, Humbolt University, Berlin, Germany, ${ }^{3}$ Zlotowski Center of Neuroscience, Ben Gurion University, Beer Sheva. Israel

Perturbations in the integrity of the Blood-Brain-Barrier (BBB) have been reported in both humans and animals under numerous pathological conditions. However, the effect of $\mathrm{BBB}$ opening on structure and function of the cerebral cortex is not
known. In human patients we found that persistent $B B B$ disruption may be detected in neurological patients, and was found to be associated with clinical symptoms consistent with partial seizures and focal EEG slowing. To study directly the role of compromised $\mathrm{BBB}$ on cortical function we focally induced $\mathrm{BBB}$ disruption using low concentrations $(<3 \mathrm{mM})$ of the sodium salts, dehydrocholic acid (DHC) or 7-deoxycholic acid (DXC). Topical application was done under anaesthesia following opening a cranial window over the somatosensory cortex of the rat. Sham-operated rats were exposed to a similar surgical procedure but perfused with artificial cerebrospinal fluid BBB disruption was confirmed using fluorescent microscopy and image analyses following the injection of the non-permeable peptide fluorescein isothiocyanate or the albumin-binding dye Evan's blue. Bile salts application did not induce signs of immediate neuronal toxicity in physiological experiments in-vitro nor in histological sections 24 hours after in-vivo application. Intra- and extracellular recordings in-vitro revealed focal paroxysmal hypersynchronous activity evoked in BBB-disrapted cortex. This abnormal activity observed in $>90 \%$ of treated slices and was rarely $(<15 \%)$ recorded in slices from sham-operated rats. Paroxysmal activity was distributed over all cortical layers, could be trigger from neighboring cortical columns but was always limited to the treated region. Pharmacological experiments suggest enhancement of both excitatory and inhibitory transmitter systems in the evoked paroxysmal responses. Our data suggest that persistent $\mathrm{BBB}$ opening may result in cortical dysfunction and epileptiform activity.

\section{Attenuation of amyloid- $\beta$ pathology by a lipophilic transition-metal chelator, DP-109, in organotypic bippocampal brain slices and human amyloid precusor} protein transgenic mice

Friedman J.E. Koh J.Y. ${ }^{3,4}$, Hoffman K. B. ${ }^{1}$, Lynch $\mathrm{G}^{2}$ and Kozak A.

${ }^{7}$ Thuris Corp. ${ }^{2}$ Univ. CA Irvine, ${ }^{3}$ Univ. Ulsan Col. Med.,

${ }^{4}$ COS Biotech., Inc. Seoul.Korea; ${ }^{5}$ D-Pharm Ltd. Rehovot,

Israel

Alzheimer's disease (AD) is the most common neurodegenerative disease causing progressive dementia. The disease is characterized by extracellular amyloid plaques. formation of neurofibrillary tangles and by activation of cellular components of inflammation. A significant body of evidence indicates that zinc and copper ions contribute to aggregation of $A \beta$ peptides, deposition of amyloid plaques and subsequent production of reactive oxygen species and activation of reactive microglia. We examined whether the lipophilic transition metal chelator DP-109 can inhibit these events in both in vitro and in vivo systems, i.e. organotypic rat hippocampal slices and transgenic Tg2576 mice. Organotypic slices were exposed to $A \beta 1-42$ in the presence or absence of DP-109 and/or the integrin antagonist, RGD, which enhances $A \beta$ uptake and subsequent microglial activation. Slices were immunostained for A $31-42$ or reactive microglia (ED1), or stained with Fluoro-Jade $B$ (FJB) to detect degenerating neurons. EDI and Ax staining were markedly increased in slices treated with $A x+R G D$ compared to $A \beta$ alone (both $p<0.001)$ DP-109 dose-dependently reduced the effects of $A \beta+R G D$ on $E D 1$ and $A \beta$-stained particles and particle area in doses ranging from 0.1 to $8 \mu \mathrm{M}(\mathrm{p}<0.01)$. DP-109 also reduced neuronal death elicited by $A \beta+R G D$, as shown by decreased FJB-stained particles $(\mathrm{p}<0.05)$. In vivo, aged female hAPP-T 2576 mice treated with DP-109 $(5 \mathrm{mg} / \mathrm{kg})$ by daily gavage for 3 months had a markedly reduced burden of amyloid plaques as compared to animals receiving vehicle trealment $(21.6 \pm 15.1$ vs. $126.1 \pm 39.5$ plaques/section, $p<$ 0.001 ). Moreover, the cerebrum area loaded with amyloid plaques was decreased by $81 \%$ in DP-109-treated mice $(p<$ $0.001)$. DP- 109 treatment also facilitated the transition of $\mathrm{A} \beta$ from insoluble to soluble forms in the cerebrum. Thus, DP-109 reduced inflammation, amyloid uptake and neuronal death in an in vitro model of $A B$ toxicity as well as reducing the plaque load in a transgenic model of AD. These results support the hypothesis that endogenous metals are involved in the deposition of aggregated $A \beta$ in brain of $A D$ patients and that lipophilic metal chelators may be useful therapeutic agents in the treatment of $A D$ and other neurodegenerative diseases.

Support Contributed By D-Pharm Ltd

A McGurk effect in schizophrenia: Audio-visual integration is altered by visual attention Fromovitz $\mathrm{M}^{\mathrm{1}, 3}$, Modai $\mathrm{I}^{1,2},{ }^{1,}$, Nahshon $\mathrm{I}^{3}$ and Peled A. ${ }^{1,2}$ 'Institute for Psychiatric Studies, Sha ar Menashe Mental Health Center. Mobile Post Hefer 38814. Hadera: ${ }^{2}$ Bruce Rappaport Faculty of Medicine, Technion. Israel Institute of Technology. Haifa; ' Dept. of Criminology Bar Ilan University. Ramat Gan

The illusion of McGurk is obtained when conflicting audio-visual messages are simultaneously presented. The McGurk illusion is one approach to study audio-visual integration in the brain. Schizophrenia has been described as a disease of neural integration; specifically auditory visual integration may be disturbed in patients with positive 
symptoms such as hallucinations. In this study we show altered auditory visual integration in schizophrenia patients compared to controls. The audio-visual stimulus was constructed from a video showing lips pronouncing a set of syllables and incongruent conflicting auditory visual stimuli were "planted" into the set of regular coherent stimuli. Generation of McGurk effect by the conflicting stimuli was measured. Between groups and within group comparisons showed that within the schizophrenia patients group the McGurke effect increased significantly when subjects attend to the visual modality of the stimulus.

\section{Oscillations of ADNP in the arcuate nucleus during the}

estrus cycle ${ }^{\text {Furman } S .}{ }^{1}$, Hill JM. ${ }^{2}$, Hauser JM. ${ }^{2}$, Vulih I. ${ }^{1}$., Brenneman $\mathrm{DE}^{2}$ and Gozes I.

${ }^{I}$ Clinical Biochemistry, Sackler School of Medicine, Tel-Aviv University, Tel-Aviv, Israel, ${ }^{2}$ SDMP, NICHD, NIH, Bethesda, $M D, U S A$.

Our laboratory recently cloned the human activity-dependent neuroprotective protein (ADNP) cDNA. ADNP contains 9 zinc fingers, a proline rich region, a nuclear bipartite signal, a nuclear export signal and a homeobox domain profile, suggesting a transcription factor function. ADNP is a highly conserved vasoactive intestinal peptide (VIP) responsive gene that is expressed abundantly in the brain and in the body. As VIP shows sexual dichotomy in the hypothalamus, the potential differential expression of ADNP in male and female mice was investigated. The expression of ADNP was also evaluated at different stages of the female estrus cycle. Northern blot hybridization of mouse hypothalamic RNA identified increased ADNP mRNA expression in the female as compared to the male. Furthermore, oscillations in ADNP mRNA expression levels during the estrus cycle were observed. Immunohistochemical staining using antibodies directed against the $\mathrm{N}$-terminal region of ADNP revealed ADNP-like immunoreactivity in the arcuate nucleus. Like ADNP mRNA, ADNP-like immunoreactivity changed with the estrus cycle, peaking at proestrus and exhibiting minimal expression during estrus. Even during estrus, the female ADNP levels were higher than those found in males. Many factors play a role in the control of the rodent female estrus cycle and show cyclic oscillations with the estrus cycle in the arcuate nucleus. The arcuate nucleus drives reproductive cyclicity through the hypothalamic pulse generator, where gonadotropin-releasing hormone $(\mathrm{GnRH})$ is regulated by afferent neuronal systems that are sensitive to estradiol and
progeseterone. One factor that affects GnRH neurons is VIP from the suprachiasmatic nucleus and the results presented here suggest that ADNP is one of the factors that regulate the estrus cycle, possibly being affected by VIP.

Support: The combined program of the NIH and the Sackler Faculty of Medicine in Women's Health, BSF, the Neufeld award, the Lily and Avraham Gildor Chair.

\section{Acquisition and extinction of memory in virtual} environment

Furst $\mathrm{E}$. and Dudai $\mathrm{Y}$

Dept. of Neurobiology, Weizmann Institute of Science, Rehovot Our knowledge of the processes and mechanisms of memory extinction stems mostly from studies in laboratory animals, and only little is known about memory extinction in the human brain. A paradigm that was occasionally used in investigating of experimental extinction in humans is the eyelid reflex membrane. This choice has been influenced by the advances in analyzing classical conditioning of eyelid in the cat and the nictitating membrane in the rabbit. Classical conditioning, however, is a primitive type of memory and, moreover, being a fear conditioning type of paradigm, is inconvenient for routine use in humans. We have set out to develop behavioral learning paradigms that could permit the analysis of experimental extinction, yet do not rely on punishment as a reinforcer and, furthermore, are more representative of the complexity of human behavior. Toward that end, we have developed a virtual environment paradigm, in which the subject has to accomplish a task (room painting) using behavioral rules and causative associations that are utterly irrelevant to the real world (e.g. turn on a TV set to mix colors). This ensures that each subject is indeed a tabula rasa at the beginning of learning as far as the rules to be learned are concerned. The acquired rules are then changed without notification and the subject has to abandon the previous rule to succeed in the task. In a third phase, the rules are switched again: either back to the first set of rules, or to a new set. Subjects were found to relearn the first set faster than learning a new one. These results indicate that the original trace was not erased, but rather underwent a relearning, extinction-like process.

Supported by the Israeli Science Foundation and the

Volkswagen Foundation.
Endocannabinoids and hepatic encephalopathy Gabbay E. Avraham $Y^{1}{ }^{1}$, Zolotarev $O_{1}^{1}$, Okun $A^{1}$, Zilberman ${ }_{1}^{1}{ }^{1}{ }^{2}$ Fink N. ${ }^{1}$, Ganzburg V. ${ }^{1}$, Y israeli E. ${ }^{3}$, Ilan Y. ${ }^{3}$, Mechoulam $\mathrm{R}^{2}$ and Berry E.M

Dept. of Human Nutrition ${ }^{1}$, Medicinal Chemistry ${ }^{2}$, Liver disease, Hebrew University, Hadassah Medical School Jerusalem.

Hepatic Encephalopathy (HE) is a reversible impairment in neurological function due to liver disease. Its pathogenesis involves: raised levels of ammonia, gamma-aminobutyiric acid and functional changes in the: opiodergic, serotonergic and dopaminergic systems.

Endocannabinoids (ECs) are neuro- and immune modulators, found in the central nervous, gut and immune systems that function via specific receptors, affecting neurological and cognitive function, appetite and mood.

Studies of chronic liver disease in animals and man have demonstrated increased levels of (ECS) in peripheral blood cells. (ECs) were also involved in some of the hemodynamic changes occurring in cirrhosis however their possible role in hepatic encephalopathy has not been explored. We have shown increased levels of the endocannabinoid 2AG in the brain of cirrhotic animals.

Our study aimed at exploring a possible role for (ECs) in the pathogenesis of $(\mathrm{HE})$. The study involves a model of fulminant hepatic failure in mice, induced by intraperitoneal injection of the hepatotoxin thioacetamide (TAA) vehicle or CBI antagnist were daily administered. Neurological and cognitive function were determined.

The neurological and cognitive performance of mice administered TAA with SR141716A (ECantagonist). improved more rapidly than that of mice given TAA alone. This finding along with the finding of elevated $2 \mathrm{AG}$ levels in animal models of both acute and chronic liver disease suggests that therapy based on modulation of the CBI antagonist function may be feasible.

Administration of the antagonist to the CBI receptor may alleviate both the neurological and hemodynamic derangements, associated with liver disease.

Selective initiation of sacrococcygeal rhythmic patterns by alphal-adrenoceptors agonists in spinal cords of neonatal rats

Gabbay H. and Lev-Tov A

Dept. of Anatomy \& Cell Biology, The Hebrew University Medical School, Jerusalem, Israel

The ability of neurochemicals to selectively activate rhythmogenic networks in the sacrococcygeal (SC) cord was examined in isolated spinal cords of neonatal rats. Fast alternating left-right rhythmic activity was produced in the SC and rostral-lumbar segments by bath-applied noradrenaline (NA). The rhythm slowed down rapidly and blocked

The fast rhythm could be produced and maintained for up to $60 \mathrm{~min}$ by the alphal-adrenoceptor agonist methoxamine and blocked by the antagonist prazosin, the rhythm was blocked in surgically detached thoracolumbar cords, but it persisted in isolated SC cords in the presence of the NMDA receptor-antagonist APV. A slow non-locomotor rhythmic pattern accompanied the fast methoxamine-induced rhythm. This rhythm persisted in detached thoracolumbar cords and it could be blocked by bath applied APV.

Injection of current steps into L2 and S2 motoneurons revealed a $30-40 \%$ decrease in input-resistance $\left(R_{N}\right)$ during troughs of the fast rhythmic drive. The reduced $R_{N}$ coincided with contralateral efferent-bursts. The rhythmic fluctuations of $R_{N}$ in $\mathrm{L} 2$ motoneurons were abolished, but the alternating left-right pattern of the efferent bursts was unchanged in midsagittally split thoracolumbar cords. These intracellular studies suggested that crossed-inhibitory pathways controlled the decrease in $R_{N}$ that activation of these inhibitory pathways in rostral-lumbar segments during the rhythm depended on intact thoracolumbar commissures, and that sacrococcygeal projections maintained the alternating pattern in midsagittally-split thoracolumbar cords.

In summary, activation of alphal-adrenoceptors exhibits differential effects on spinal neural networks; it initiates pattern generation by the SC circuitry, and it modulates the NMDA receptor-mediated rhythmicity of the thoracolumbar pattern-generators. 
Molecular study of neurodevelopmental animal model of schizophrenia, as revealed by the analysis of hippocampal and striatal gene expression profiles by means of cDNA microarrays and Real-Time PCR

Gak E. ${ }^{1,2}$, Koronyo-Hamaoui M. ${ }^{1,2}$, Zuckerman $\mathrm{L}^{3}$ and Weiner ${ }^{1}$ Danek Gertner Genetics Institute, Sheba Medical Center; ${ }^{2}$ Sackler Medical School and ${ }^{3}$ Dept. of Behavioral Sciences, Tel Aviv University, Tel Aviv

A novel neurodevelopmental model of schizophrenia in rats developed by Zuckerman and Weiner 2002 demonstrated that prenatal administration of synthetic cytokine releaser poly I:C that elicits maternal immune activation, leads to maturation-dependent disrupted latent inhibition mimicking that found in schizophrenia, as well as neurochemical and morphological alterations consistent with pathophysiology of schizophrenia. We presently report gene expression alterations in two relevant brain regions of post-pubertal poly $\mathrm{I}: \mathrm{C}$ treated offspring using cDNA microarray and gene-specific quantitative PCR analyses. Our most prominent finding is 5-fold elevated expression of hippocampal secretogranin 11 and 2.5-fold of striatal secretogranin $V$ in poly I:C treated rats, that are reproduced by quantitative PCR. Apart from their established role in secretory granule formation in neuroendocrine and nervous tissues, secretogaranins were also neuroendocrine and nervous tissues, secretogaranins were also hippocampal finding, is 4-fold reduced expression of catalitic $\mathrm{Na} / \mathrm{K}^{+}$ATPase that is well-documented in schizophrenia, together with mitochondrial cytochrome-c oxydase COX IV that is reduced in hippocampal and striatal poly I:C. Several G-proteins involved in intracellular signal transduction are consistently elevated in hippocampal and striatal poly I:C. G-proteins and their regulators were demarcated by recent microarrays analyses of schizophrenic brain. The myelin basic protein, an extracellular neuronal matrix component that conrtibutes to neural connectivity and plasticity, is elevated in the poly I:C hippocampus and striatum, although marginally by quantitative PCR standards. These findings may indicate directions along which the poly I:C model might be further explored and substantiated as an authentic embodiment of neurodevelopmental processes underlying schizophrenia.

Wasp uses specialized sensors to probe and inject venom inside the brain of its cockroach prey

Gal R., Haspel G. and Libersat F.

Dept. of Life Sciences and Zlotowski Center for Neuroscience, Ben Gurion University, Beer-Sheva

The parasitoid wasp Ampulex compressa stings a cockroach, Periplaneta americana directly into the two head ganglia: the supraesophageal (brain) and the subesophageal (SEG) ganglia. This sting is precisely targeted and is followed by the injection of a venom cocktail, which causes the cockroach to become hypokinetic for the next 3 weeks

We hypothesize that the wasp locates the brain and SEG inside the cockroach head with specialized sensors on its stinger to discriminate nervous tissue from non-nervous tissue inside the head cavity of the cockroach. Using Scanning Electron Microscopy we identified at least 2 different types of sensilla-like structures on the wasp's stinger. Using retrograde staining from the cut tip of the stinger we identified the projections of sensory neurons reaching from the stinger's tip to the last abdominal ganglion of the ventral nerve cord. The staining revealed approximately 20 axons in 2 pairs of nerves. To find whether the wasp can discriminate nervous from non-nervous tissue, we let wasps sting cockroaches with the brain or SEG removed. We measured the duration of the stinging sequence in each case, and compared it to sham-operated cockroaches, with no neuronal lesions. The duration of the stinging sequence increased significantly when wasps stung cockroaches with the brain or SEG removed (8-fold and 4-fold, respectively). In a separate set of experiments, we inactivated the cuticular receptors on the wasps' stinger by applying liquid nitrogen on its tip. We then let these wasps sting untreated cockroaches. The stinging duration increased 10 -fold after freezing the stinger

Thus $A$ compress $a$ appears to use a sensory feedback from specialized cuticular sense organs located on its stinger while stinging the cockroach. The sensory receptor neurons associated with these cuticular sense organs are able to discriminate nervous from non-nervous tissue. This enables the wasp to precisely locate the brain and the SEG inside the cockroach head.
The problem of spike-timing-dependent-plasticity for distal dendritic synapses

Gidon A.A. and Segev I.,

Tinstitute for Life Science, Hebrew University Jerusalem,

${ }^{2}$ Interdisciplinary Center for Neural Computation

The spike timing dependent plasticity (STDP) "Hebbian" mechanism has been shown to operate in synapses of many neuron types. When the post-synaptic cell fires a spike within a narrow time-window, following an excitatory input, the conductance of that synapse increases (potentiation). If the post-synaptic cell fires before the pre-synaptic cell, the synaptic conductance is decreased (depression). As long as the total depression is larger than total potentiation there is effectively, a competition between synapses over the control of post-synaptic firing. The degree of competition is determined by the ratio of the depression to the potentiation parts of the STDP function. Simulations show that this competition results in subgroups of synapses that become stronger whereas the rest of the synapses get weaker.

When implementing STDP in a dendritic neuron model with synapses that receive random asynchronous input, the following problem arises. Due to cable filtering, distal synapses evoke attenuated somatic EPSPs and, thus, their efficacy in generating a spike is low. Consequently these synapses are weakened due to the STDP mechanism. Starting with a uniform distribution of synaptic strength, very soon distal synapses are effectively extinct whereas proximal synapses become maximally strengthened. This distribution of synaptic strength however, is not found in in vitro experiments. On the contrary, in some cases distal synapses seems to be stronger than proximal ones.

Here we propose several possible STDP-based local mechanisms that solve this contradiction. One possibility to partially overcome the weakening of distal synapses is to implement a biologically plausible multiplicative model, whereby the modification of a synapse is inversely proportional to its current strength. Other possible mechanisms that equalize the conductance change of distal versus proximal synapses are also discussed.

Novel anti-psychotics that display GABAergic activity and decreased extrapyramidal side effects, for the treatment of schizophrenia and related psychiatric disorders

Gil-Ad $1{ }^{1}$, Rephaeli A. ${ }^{2}$, Weizman $A^{1}{ }^{1}$, Nudelman $A^{3}$

'Lab of Biological Psychiatry, Experimental Oncology,

Felsenstein inst., Tel-Aviv University, Tel Aviv, ${ }^{3}$ Dept. of

Chemistry, Bar Ilan University, Ramat Gan

Neuroleptics are widely used drugs in the treatment of schizophrenia and related disorders. Current drugs induce undesirable side effects such as extra pyramidal symptoms for typical drugs and weight gain and metabolic disturbances for atypical agents. Gamma-amino butyric acid (GABA) is the major inhibitory neurotransmitter in the brain. Recent reports, suggested that GABA deficiency is involved in the pathogenesis of schizophrenia. We synthesized the novel molecules AN-168 and AN-187 and found they display neuroleptic as well as GABAergic activities. Their effect on catalepsy (in an extrapyramidal model), sedation and prolactin release (marker of DA receptor blockade) in young adult male rats or mice following ip or po administration $(2.5-20 \mathrm{mg} / \mathrm{kg})$, was evaluated. Comparison between AN-168 and AN-187 to typical neuroleptics, using equivalent doses and the same parameters, was conducted side by side. Catalepsy was measured hourly for $6 \mathrm{~h}$. At clinically relevant doses, while the typical neuroleptics induced significant catalepsy, these compounds did not induce catalepsy, but did elicit mild sedation. At high doses, they induced mild catalepsy, while typical neuroleptics brought about severe catalepsy. At equivalent concentrations, our compounds and typical antipsychotic drugs stimulated prolactin release to the same extent following the same time course. While AN-168 and AN-187 exerted GABA agonistic and classical DA(D2) antagonistic activities, their extrapyramidal side effects following treatment were markedly diminished. In conclusion, the data suggest, that the novel agents display GABAergic as well as neuroleptic activities and therefore may constitute a novel type of neuroleptics. 
Lysyl oxidase, the extracellular matrix-forming enzyme, in amyloid plaque formation

Gilad G.M. ${ }^{1}$, Kagan H.M. ${ }^{2}$ and Gilad V.H. ${ }^{1}$

TResearch and Development, Laboratory of Neurascience, Assaf Harofeh Medical Center, Zrifin 70300, Israel; ${ }^{2}$ Dept. of Biochemistry, Boston University School of Medicine, Boston. $M A, U S A$

Alzheimer's disease (AD) is characterized by the wide spread formation of extracellular senile plaques in the human forebrain. Amyloid beta protein polymer deposits, the primary constituent of plaques, were found to be associated with various extracellular matrix (ECM) proteins in the plaques, but the precise mechanisms of plaque formation are not yet resolved. The present study is based on the premise that the enzyme lysyl oxidase (LO), which catalyzes the crosslinking of ECM proteins and formation of insoluble matrices, is secreted by cells at the sites of plaque formation and participates in ECM modulation, thereby contributing to the progressive pathology in AD. Experiments were performed on autopsy brain samples taken from the hippocampus region of $\mathrm{AD}$, non-Alzheimer's dementia and a control group of unrelated diseases. The results indicate that $L O$ enzyme activity is increased in $A D$, but also in non-Alzheimer's dementia as compared to controls. Immunocytochemical staining with specific LO antibodies indicates that the enzyme is localized in blood vessel walls and, in the brain parenchyma, it is associated with plaque formations some of which positively stained with thioflavin-S. The observed number of LO-positive plaques in AD was over 2-fold higher as compared to both non-Alzheimer's dementia and control groups. These findings indicate that active LO molecules are associated with senile plaques, thus providing solid support for the novel hypothesis implicating LO in ECM modulation associated with plaque formation in $\mathrm{AD}$.

\section{Agmatine can potentiate and attenuate}

1-methyl-4-phenyl-1,2,3,6-tetrahydropyridine

dopaminergic toxicity

Gilad V.H. ${ }^{1}$, Gilad G.M. ${ }^{1}$ and Rabey J.M. ${ }^{2}$

Laboratory of Neuroscience, Research and Development and 'Dept. of Neurology, Assaf Harofeh Medical Center, Zrifin

Treatment with agmatine, decarboxylated arginine, proved to be nontoxic and to exert potent neuroprotective effects in several models of neurotoxic and ischemic brain and spina cord injuries. Here we sought to determine whether agmatine treatment would also prove beneficial in the mouse 1-methyl-4-phenyl-1,2,3,6-tetrahydropyridine (MPTP) model of Parkinson's disease. MPTP-treated 3-4-month-old C57BV/6 mice (40 mg/kg intraperitoneal, once daily for 2 days) were subjected to agmatine treatment $(100 \mathrm{mg} / \mathrm{kg}$ intraperitonel once daily) commencing either 1 hour prior to the $1^{\text {st }}$, or 8 hour after the last MPTP injection and lasting until the $5^{\text {th }}$ MPTP after the last MPTP injection and lasting until the 5 MPTP
post-injection day. Dopamine (DA) uptake by synaptosomal preparations from the striatum served as a functional marker of dopaminergic axon terminals. Agmatine did not affect synaptosomal DA uptake, but aomatine treatment prior to Synaptosomal DA uptake, but agmatine treatment prior to in pmol $/ \mathrm{mg}$ protein $/ 5 \mathrm{~min}$ : control $=1.63 \pm 0.09(100 \%), \mathrm{MPTP}$ $=0.68 \pm 0.03(42 \%)$ and agmatine $+\mathrm{MPTP}=0.50 \pm 0.04(31 \%)]$ Moreover, prior agmatine treatment completely negated the preventive effect of the monoamine oxidase B (MAOB) inhibitor, deprenyl, against MPTP toxicity (deprenyl + MPTP $=78 \%$ vs. deprenyl + agmatine + MPTP $=38 \%$ of control). In contrast, when agmatine was applied after MPTP it produced partial protection against MPTP toxicity ( $62 \%$ of control). We conclude: 1) the coincident presence of systemic agmatine and MPTP potentiates MPTP-related dopaminergic damage even in the presence of MAOB inhibition, and 2) agmatine attenuates MPTP-related dopaminergic damage when applied well after MPTP treatment. The present findings implicate agmatine in the initial mechanisms regulating MPTP neurotoxicity, while reiterating agmatine's characteristic neuroprotective efficacy after application of the insult.

Analysis of gene expression in MOG-induced experimental autoimmune encephalomyelitis after treatment with a autolmmune encephalomyelitis afte Gilgun-Sherki Y. ${ }^{1}$, Barhum Y..$^{1}$, Atlas D. ${ }^{2}$, Melamed E. ${ }^{1}$, and Offen D.

Dept. of Neurology and Felsenstein Medical Research Center Rabin Medical Center. The Sackler School of Medicine, Tel Aviv University, Petah Tikva 49100; Institute of Life Sciences. The Hebrew University, Edmond Safra Campus, Jerusalem Accumulating data from experimental studies indicate that oxidative stress (OS) has a major role in the pathogenesis of multiple sclerosis (MS). It has been suggested that local production of reactive oxygen species (ROS), probably by macrophages, mediates axonal damage in both MS patient and the mouse model experimental autoimmune encephalomyelitis (EAE). We have previously shown that AD4 ( $\mathrm{N}$-acetylcysteine amide), our novel brain penetrating antioxidant, reduces the clinical and pathological symptoms, including inflammation and axonal damage in myelin oligodendrocyte glycoprotein (MOG)-induced chronic EAE in mice. The aim of this study was to examine the molecular mechanism by which AD4 exerts protection in MOG-induced EAE mice. Therefore, we analyzed gene-expression profile in the spinal cords of MOG-induced chronic EAE mice and compared to MOG-induced mice treated with AD4, using a cDNA microarray. We found that MOG treatment up-regulated genes encoding growth factors, cytokines, death receptors, proteases and myelin structure proteins, while MOG/AD4-treated mice demonstrated gene expression profile similar to the profile seen in naive-healthy mice. In conclusion, our study shows that chronic $\mathrm{AD} 4$ administration suppresses the induction of various pathological pathways that play a role in EAE, and probably in MS

Focal cortical dysfunction and blood-brain-barrier disruption in patients with post-concussion syndrome Golan $H^{1}{ }^{1}$, Korn A. ${ }^{2}$, Melamed I. ${ }^{2}$, Pascual-Marqui R. ${ }^{3}$ and Friedman A

Nuclear Medicine, Rabin Medical Ctr., Golda-Hasharon Campus, ${ }^{2}$ Zlotowski Ctr. of Neuroscience, Ben-Gurion University, Beer Sheva ${ }^{3}$ The Key Institute for Brain-Mind Research, Univ. of Zurich, ${ }^{4}$ Inst. for Physiology, Humboldt Univ., Berlin.

Post concussion syndrome refers to symptoms and signs occurring commonly after mild head injury. Although the organicity of the syndrome is well documented, its pathogenesis is unknown. The aim of this study was to search for sources of abnormal brain activity as revealed by quantitative electroencephalographic recordings and correlate these with imaging. Data from 17 patients following histories of mild head trauma and diagnosis of post concussion syndrome was retrospectively analyzed. Digitized electroencephalography from patients was compared to that from age-matched healthy volunteers. Low resolution electrotomography (LORETA) was used for localizing abnormal rhythms and was correlated with brain imaging. Normalized quantitative electroencephalography revealed significantly higher power in the delta band and lower power in the alpha band compared to controls. The generators for the abnormal rhythms were focally localized in distinct neocortical regions. While brain computerized tomography and/or magnetic resonance imaging did not reveal focal abnormality, single photon emission computerized tomography showed a focal reduction in cortical perfusion in $85 \%(n=11)$ of the patients, and abnormal blood-brain-barrier following Tc-DTPA in $73 \%(n=8)$. In $75 \%$ of these patients, LORETA analysis showed that the generators for abnormal rhythms were closely related to the anatomical location of blood-brain-barrier lesion. Our data point, for the first time, to focal persistent blood-brain-barrier disruption and hypoperfusion as a possible pathogenic mechanism in at least some post concussion patients.

\section{The mouse bHLH transcription factor Nato3 is a novel} floor plate-specific marker

Golan-Lev T and Ben-Arie N.

Dept. of Cell and Animal Biology, Institute of Life Sciences The Hebrew University of Jerusalem, Jerusalem 91904

The basic helix-loop-helix (bHLH) transcriptional regulatory proteins have an important role in developmental processes including neurogenesis, myogenesis, hematopoiesis and pancreatic development

Our lab has revealed a novel bHLH subfamily, which is similar to the atonal subfamily of Drosophila, and was therefore desionated nephew of atonal 3 (Nato3). Mouse Nato3 (MNato3) was found to be expressed as early as E7, and as late as $\mathrm{P} 35$ by RT-PCR.

Expression analysis in the mouse spinal cord at E11-16 by in situ hybridization revealed that MNato 3 is specifically expressed in the floor plate. In comparison to other known floor plate markers, its expression pattern is more similar to Slit2 than to F-Spondin.

Interestingly, at E13, but not at E11, the expression domain in the floor plate is wider at the caudal end and becomes restricted to the midline towards the rostral end. The expression domain at the caudal level becomes more localized to the midline with development (E13.5-E16.5)

Floor plate cells have an essential role in neural cell patterning and axonal outgrowth in the spinal cord. It is therefore suggested that MNato3 may have an important role in the development of the spinal cord.

Supported by the US-Israel Binational Science Foundation

(2001013) and the Roland Center for Neurodegenerative

Diseases. 
The dynamics of GABA $A_{B}$ leads to frequency-dependent latency in the paralemiscal response to vibrissa stimulation Golomb D, ${ }^{1,2}$ and Kleinfeld D.

Tept. of Physiology and Zlotowski Center for Neuroscience, Ben-Gurion Univ., Be'er-Sheva, Israel; ${ }^{2}$ CTBP, Physics Dept. UCSD, La Jolla, CA

The latency in the steady-state spiking response of POm thalamic neurons and cortical L5 neurons, which constitute the paralemniscal pathway, increases with increasing frequency of periodic vibrissa stimulation (Ahissar et al., Nature,2000; Ahrens et al., PNAS, 2002). In contrast, the latency of the response of neurons in VPM thalamus of the lemniscal pathway is essentially constant. We hypothesize that $\mathrm{GABA}_{\mathrm{B}}$-mediated delayed and prolonged inhibition from the reticular thalamic nucleus (RE) is an essential determinate of the latency. We test this hypothesis in terms of a firing rate model that includes excitatory thalamic and cortical neurons in both pathways and one population of inhibitory nuclear reticularis (RE) neurons that form reciprocal connections with POm and VPM cells. The GABA $A_{B}$ inhibition of RE neurons is shown to cause increasing latency with increasing frequency. The increase in latency in POm is greater than that in VPM because of the relatively stronger brainstem drive. We predict that blocking $\mathrm{GABA}_{\mathrm{B}}$ in the POm will destroy the latency effects in thalamus and cortex

Supported by the ISF, NSF, NIMH.

\section{Long term effects of organophosphates exposure, stress} and their interaction.

Grauer E and Kapon J.

Dept. of Pharmacology, Israel Institute for Biological

Research, Ness Ziona.

A possible interaction between stress and organophosphate (OP) exposure was suggested based on long term follow up of victims of the sarin terrorist attacks in Japan. This was supported by a high rate of PTSD, as well as an increase in stress-related symptoms such as anxiety, restlessness, mood shifts, sleep disturbance, depression, attention deficits and memory loss reported in these victims. The following studies were performed in an attempt to detect and characterized possible OP exposure interaction with stress processes in rats, with a special emphasis on long term effects. Experiment 1: rats were exposed to sarin $\left(0.9 \mathrm{LD}_{50}\right)$ and three weeks later were tested in a contextual fear conditioning paradigm. In this, rats undergo a session of fear conditioning followed by three successive re-introductions to the same environment. Results suggest that rats previously exposed to sarin showed decreased freezing behavior compared to controls. Experiment 2: sarin exposed rats $\left(0.9 \mathrm{LD}_{50}\right)$ were subjected to 6 week of variable unpredictable stress exposure. Following the termination of stress, rats were tested in succession of tests. Hypothermia induced by the cholinergic agonist oxotremorine $(0.5 \mathrm{mg} / \mathrm{kg}$, sc) was used to detect alterations in central cholinergic receptor sensitivity. Results suggest interaction between the effect of stress and sarin to produce an increase in the hypothermic response. Similar interaction was also demonstrated in animals' weight at the end of the study; Five months after stress termination the weight of rats exposed to either sarin or stress was lower than that of controls and the weight was further decreased by the combined exposures. Thus, the interaction between the effects of stress and those of sarin exposure may amplify the expected long-term effects of the latter to produce significant symptoms even at low doses.

Phase maintenance promoted by the interplay of synaptic and intrinsic dynamics

Greenberg 1. Merbl Y. and Manor Y

Zlotowski Center for Neuroscience \& Dept. of Life Sciences, Ben-Gurion University. Beer-Sheva 84105

Central pattern generators are neuronal networks that produce multi - phasic rhythms. In many cases, the pattern of neuronal firing activity retains an approximately constant structure despite wide changes in cycle period. In many central pattern generators, pairs of neurons maintain an approximately fixed phase, where the phase is defined as the ratio of firing interval of the two neurons and the cycle period. The mechanisms underlying phase maintenance are not clear. Previous work suggested a simple mechanism for phase maintenance in a circuit that consisted of an oscillator synaptically coupled to a follower cell. When the synapse was inhibitory and depressing, this model demonstrated that the phase between the oscillato and the follower could be approximately constant over a relatively wide range of cycle periods. At slow cycle periods, the synapse maximally recovered and its effect on the firing interval became insensitive to changes in cycle period. This effect upper- bounded the range of cycle periods for which phase could be maintained in this circuit. We extended this model and introduced a transient potassium (IA-like) current in the follower cell. With a proper choice of parameters, we were able to tune the dynamics of the IA conductance such that it delayed the activity of the follower cell only at slow cycle periods. The existence of an IA conductance did not contribute much to phase maintenance when the synapse was non-depressing. However, when synaptic depression was combined with an IA conductance in the follower cell, the interplay between synaptic and intrinsic dynamics dramatically increased the range of cycle periods for which phase was maintained.

Supported by BSF2001 - 039 (YM)

Brain activation during size invariant face recognition: $A$ functional imaging study

Grinfeld $A$. and Gur $M$.

Biomedical Engineering, Technion, Haifa

Invariant object recognition can be done by having a normalized view of the objects in memory - a normalized representation, transforming the viewed image to the size format of the stored one, and then testing for a match (Bundesen, C., \& Larsen, A. 1975).

To identify the brain structures implicated in mental transformation of size, and trace the variation in neural activity within each of these areas over space and time, we used two functional imaging methods: fMRI with a spatial resolution of several millimeters and a temporal resolution of several seconds, and LORETA- a functional imaging method based on Event Related Potentials that gives a fair degree of spatial localization at a superior millisecond resolution. Nine normal subjects compared randomly presented faces regardless of variation in size in a one-back, match- to-sample paradigm.

Here, we report different patterns of activation, primarily located in the dorsal pathways, comprising structures in the occipital, parietal, and temporal areas. Of these areas, the fusiform and inferior temporal ovri were more activated by tasks with different size stimuli than by tasks with same size stimuli.

We propose that the posterior parts of the fusiform and inferior temporal gyri, are involved in higher level processes that lead to mental transformation of size.

Effects of pre-training on performance in spatial and odor discrimination tasks

Grossberger T.', Sarig O.', Barkai E. ${ }^{2}$, Cohen $\mathrm{H}^{3}$ and

Richter-Levin G.

'Dept. of Psychology, Faculty of Social Sciences, University of Haifa.; ${ }^{2}$ Faculty of science and science education, University of Haifa; ${ }^{3}$ Faculty of Medicine, Ben-Gurion University of the Negev.

It is well established that learning new tasks is influenced by proceeding experiences, as in pro-active and retroactive interference. However, phenomena of post-training enhancement are described as well. These phenomena do not indicate transfer of knowledge from one task to another; rather, they implicate a change in the ability to learn a new task. Such a change may be of interest to the field of rehabilitation, in which there's an effort to generalize abilities from treatment to everyday life.

Here, we studied the enhancement of performance in simple learning tasks following learning of other tasks, while manipulating two parameters: 1) nature of task (i.e. spatial vs. odor discrimination), and 2) the motivation to perform the task (i.e. escape learning vs. appetitive learning).

In order to examine these parameters experimentally, we have used and designed four learning tasks: the Morris water maze (a spatial task in which the motivation is to escape the water), the Radial maze (a spatial task in which the motivation is to drink water), an odor discrimination task in the water maze, in which the rat uses odor to escape the water, and an appetitive odor discrimination task (in which the motivation is to drink).

In the present paradigm, rats were trained in one task and immediately afterwards learned another task, so the transfer from one task to another was examined in 12 pairs of tasks. Performance in the second task was compared between pre-trained groups, pseudo pre-trained groups and a najve group.

Results show enhancement of the ability to learn spatial and odor discrimination tasks following learning of appetitively motivated tasks, regardless of their nature. Presently we examine the transfer of ability from any escape task to the other tasks. 
Study of oxidative stress markers in a tissue culture model of stretch injury

A. ." Shohami E. ${ }^{1}$. and Kohen R. ${ }^{2}$

Dept. of Pharmacology and ${ }^{2}$ Dept. of Pharmaceutics.

Hebrew University, School of Pharmacy, Jerusalem, Israel

Background: Oxidative stress is one of the deleterious effects that increases the primary damage after mechanical or ischemic damage. It is a result of massive production of reactive oxygen species (ROS) that exceeds the capacity of the endogenous antioxidants to neutralize them. We have adopted a model system for stretch injury in culture and investigated the levels of nitric oxide (NO) and the antioxidant uric acid (UA), as well as the activity of their synthesizing enzymes: nitric oxide synthase (NOS) and xanthine oxidase (XO). Methods: Spontaneously transformed epithelial cell line (HaCaT) was cultured in wells with a flexible silastic bottom. A cell injury controller was used to produce a rapid pressure of known amplitude and duration leading to a stretch injury which deforms the cells and serves as our model system. Medium was sampled at 9-17 h. UA level was measured by HPLC-EC, NO by Griess reaction, and cell survival by MTT. The effects of the inhibitors Oxopurinol (OP for XO) and NW-Nitro-L-Arginine Methyl Ester (L-NAME for NOS) were studied. Results and Discussion: NO accumulated in the medium between 9-17h after injury to a significantly higher levels than in the control culture, $(p<0.0001)$ indicating ROS production. This was also evidenced by a ROS-sensitive fluorescent dye. At the same time, UA levels, which progressively increased in the control, remained constant in the injured culture, and were significantly lower than in the controls, suggesting consumption of UA by NO. MTT results indicate about $50 \%$ cell death after injury. When L-NAME, OP or both were added to the culture after injury a sionificant reduction in NO and UA were observed $(\mathrm{p}=0.0049$ and $\mathrm{p}=0.0004$ ) yet, these treatments did not affect cell survival. Our findings suggest that stretch injury activates NOS and XO, however, their role in cell death after injury needs to be further explored.

\section{Electroretinographic characteristics of the albino rat} model: Restoration of visual pigment

Gurshumov N. and Yinon U.

Physiological Laboratory, Goldschleger Eye Research Institute. Tel-Aviv University Faculty of Medicine, Sheba Medical Center, Tel-Hashomer

Albino mammals have several structural and physiological abnormalities, resulting in impaired visual functions. A controversy, however, exists with regard to the visua sensitivity of albino animals. The present study had characterized the physiological process of visual pigment restoration in albino rats as compared to pigmented rats. 15 albino (Wistar) and 15 pigmented $(D A)$ adult rats were studied. The retinae were exposed to high intensity light source for a period of 15-20 minutes. The retinal dark-adaptation process, representing the visual pigment's restoration process was observed by a series of flash electroretinographic (ERG) recordings during an additional period of $45 \mathrm{~min}$ in the dark The results indicate no significant difference $(P>0.05)$ in the rate of b-wave and a-wave restoration between the albino and pigmented rats with regards to their amplitude. However, significant $(P<0.05)$ differences were found between the albino and pigmented groups with regard to the latency parameters; a latency values were longer in albino rats

We concluded that despite the fat that the amplitude of a- and b-waves in albino and pigmented rats restores almost identically, the latency parameters of the ERG represent an important characteristic of the albino rats. The longer latency in the albino rat shows a delayed procedure, which may indicate a difference either in their photopigment quality or in the neural processing.

Control of IPSI- versus contra-lateral axonal turning of D1 proprioceptive interneurons

Guy U. Rinski L and Klar A

Dept. of Anatomy and Cell Biology, Hebrew University Hadassah Medical School, Jerusalem

Understanding the neural organization of the spinal cord, and the way in which its circuits integrate proprioceptive and descending neural activity to produce movement has challenged neuroscientists for over a century. Although there are many well-studied populations of spinal interneurons, there is still much to be learnt about the roles and cellular properties of interneurons in sensory-motor pathways.

The avian embryo has many advantages for studying vertebrate embryonic development, and axon pathfinding. The embryo is flat, develops in ovo, and accessible for surgical manipulations. We have developed a method of cell type-specific expression using specific enhancers, in the chick embryonic spinal cord. Mathl is a bHLH transcription factor, expressed in the most dorsally located interneurons in the spinal cord. Mathl govern the development of multiple components of the proprioceptive pathway. Utilizing the Mathl enhancer to drive expression of reporter genes, we studied Dl axonal trajectory. D1 neurons give rise to two subpopulations of neurons. One subpopulation projects axons toward the ventral midline, and eventually crosses to the contra-lateral side of the spinal cord, while the other projects ipsi-laterally. The contra and ipsi-lateral axon branches, fasciculate together at the lateral funiculus. The possible role of LIM homeodomain protein, and guidance molecule, in controlling the contra versus the ipsi decision will be presented.

Assembly of a FET-enzyme bioelectronic hybrid system for the detection of acetylcholine esterase activity

Hai A. Shappir Y. Yitzhaiek S. ${ }^{3}$ and Spira ME

${ }^{T}$ Depts. of Neurobiology, ${ }^{2}$ Engineering and ${ }^{3}$ Chemistry. The Hebrew University of Jerusalem

We have been successful in assembling a highly sensitive sensor for agonist and antagonist of acetyl choline esterase (AchE). The biosensor is based on molecularly modified field effect transistors (FET) to which AchE was covalently linked. The link was formulated by a small and highly conjugated organic coupling agent. The anchoring of AchE by this method does not modify the enzyme's properties. Bathed in water, buffer or ionic solution the enzyme maintains its catalytic activity for weeks. At present the sensor detects $10^{-3} \mathrm{M}$ to $10^{-8}$ $\mathrm{M}$ acetyl choline (Ach) in the above-mentioned solutions. The response time of the sensor at room temperature $\left(\sim 24^{\circ} \mathrm{C}\right)$ is shorter than one sec. The response is totally reversible. The catalytic activity of the enzyme is blocked by AchE antagonists (such as eserine) at physiological concentrations. The Biosensor was developed for use in both water and air interfaces.

Immunological changes induced during antisense oligodeoxynucleotide treatment in experimental autoimmune myasthenia gravis

Hamra Y. Sicsic C. and Brenner T.

Dept. of Neurology Hadassah Medical Center, Jerusalem

Myasthenia gravis is an antibody-mediated, autoimmune neuromuscular disease in which the nicotinic acetylcholine receptor (AChR) is the major autoantigen. The typical neuromuscular junction symptoms can be transiently alleviated by acetylcholinesterase (AChE) inhibitors (such as pyridostigmine). Previously we found that long-term treatment of experimental autoimmune myasthenia gravis (EAMG) rats with antisense oligodeoxynucleotides suppressing muscle AChE biosynthesis (EN101), improved muscle activity as well as clinical symptoms of the disease. The aim of the present study was to determine whether the beneficial influence of EN101 treatment on the clinical outcome of EAMG results from amelioration of the immunological processes. Repeated administration of EN101 for a month reduced the anti-rat AChR antibody levels by $90 \%$. In addition, incubation of both human and rat lymphocytes with EN 101 resulted in a dose response reduction in the proliferation rate. In the in vitro experiments, EN101 also lowered total IgG antibody production by spleen lymphocvtes. Our results show the production by spleen lymphocytes. Our results show the immunological parameters and highlight the potential advantage of gene-targeted drug therapy.

Cognitive functioning and insomnia among elderly people Hanuka E. '. Spira T. ', Hadad B. ', Breznitz Z. ${ }^{2}$, Shefer O. ${ }^{2}$ Breznitz N. ${ }^{2}$ and Haimov I.

'Dept. of Behavioral Science. Emek Yezreel Academic College: ${ }^{2}$ CogniFit: Mind Fitness Solutions. Tzipori

Chronic insomnia and cognitive impairment are both common complaints among older adults. Even so, only a few studies have examined the effects of chronic insomnia on cognitive functioning among the elderly, and the results of these studies are contradictory. We therefore examined whether insomnia is associated with changes in cognitive functioning among elderly people.

The study population comprised two groups: 63 elderly subjects without sleep disorders, and 35 elderly insomniacs exhibiting actigraphically confirmed decreases in sleep efficiency. There was no significant difference between the two groups in age and in computer skills.

The cognitive capacity of each subject was tested at the subject's home using the computerized "MindFit" test (CogniFit, Inc.). This enabled us to monitor cognitive performance and sleep in an objective way, under natural circumstances, and with minimal distortions. 
The test consisted of eight different tasks: (1) memory span, including digit and spatial span; (2) allocating attention to a target, with and without distraction; (3) selective attention, with Stroop-like interference; (4) psychomotor skills, i.e., tracking; (5) time estimation, both auditory and visual; (6) executive functioning-planning and working memory; (7) naming; and (8) integration of two dimensions-visual and semantic.

A preliminary analysis demonstrated that in five categories of cognitive functioning, elderly people without sleep disorders displayed better performance results compared to insomniacs. More specifically, the results revealed significant differences between insomniacs and good sleepers on memory span, allocating attention to a target, time estimation, working memory and integration of two dimensions.

Overall, the findings imply that, at least in some aspects, sleep disturbances may account for cognitive decline in the elderly.

\section{Axoplasmic importins enable retrograde injury signaling in lesioned sciatic nerve}

Hanz S. , Perlson E. ', Willis D. ${ }^{2}$, Zheng J-O. ${ }^{2}$, Huerta J.J. ${ }^{3}$,

Massarwa R. ${ }^{1}$, Kohler $\mathrm{M}^{4}$, van-Minnen J. ${ }^{3}$, Koltzenburg $\mathrm{M}^{3}$,

Twiss J.L. ${ }^{2}$, and Fainzilber $M$.

${ }^{I}$ Dept. of Biological Chemistry, Weizmann Institute of Science, Rehovot, Israel; ${ }^{2}$ Neuroscience Research Laboratory, Al duPont Hospital, Wilmington, DE 19899; Institute of Child Health, University College London, United Kingdom; ${ }^{4}$ Max Delbrueck Center, Charite, Berlin, Germany; 'Vrije

Universiteit Amsterdam, The Netherlands

Ambron and colleagues have shown that Aplysia axonal proteins containing nuclear localization signals (NLS) traffic retrogradely to the cell body following injury, suggesting that such 'retrograde injury signals' are required for successful neuronal regeneration (Mol. Neurobiol. 13, $61-79$ [1996]). We have examined this hypothesis in the mammalian sciatic nerve/dorsal root ganglia (DRG) system, and based on these studies can now propose a general mechanism for retrograde injury signaling in nerve axons. Nuclear import factors from the importin/karyopherin $\alpha$ and $\beta$ families are found in axons in the sciatic nerve, at significant distances from the cell body. The expression level of importin $\alpha$ did not change after lesion, whereas in contrast importin $\beta$ protein was significantly elevated, due to local translation of axonal mRNA. The newly synthesized importin $\beta$ binds to the importin $\alpha$ pre-existing in the axon thereby triggering formation of a high affinity NLS-binding complex. The complex traffics retrogradely via an association with the motor protein dynein. Use of synthetic NLS peptide as a competitor of endogenous proteins that may bind this complex inhibited the regenerative outgrowth of axotomised adult DRG neurons in culture. Moreover, application of synthetic NLS peptide concomitantly with a conditional lesioning of the sciatic nerve in vivo inhibited the subsequent enhanced outgrowth of conditioned L4/L5 DRG neurons. Thus, lesion-induced upregulation of axonal importin- $\beta$ enables the formation of an injury signaling complex and targets it for retrograde transport in injured nerve.

Theatre of the mind: functional selectivity under free viewing of audio-visual motion pictures

Hasson U. ${ }^{1}$, Nir Y. ${ }^{2}$ Levy ${ }^{1,3}$, Fuhrmann G. $^{1}$ and Malach $\mathrm{R}^{1}$ Dept. of Neurobiology. Weizmann Institute of Science, Rehovot 76100; ${ }^{2}$ Dept. of Computer Science, Tel Aviv University $613900^{3}$ The Interdisciplinary Center for Neural Computation, Hebrew University, Jerusalem 91904

To what extent is the functional organization of the human visual cortex maintained during natural vision? Here we explored this question by letting subject freely view an uninterrupted feature film. Using a new, unbiased, analysis, in which spatio-temporal activity patterns in one brain were used to "model" activity in another brain, we found a striking level of voxel by voxel synchronization in individuals watching the same movie. Surprisingly, the synchronization extended beyond primary and secondary visual and auditory areas into association cortices. The inter-subject synchronization consisted of two components: 1. A widespread cortical activation pattern correlated with emotionally arousing scenes 2. A regionally selective component, which fully recovered known cortical specializations through reverse correlation of the time-course activation peaks with the corresponding movie frames. The results reveal a surprising tendency of individual brains to "tick collectively" during natural vision. Finally, we propose the reverse correlation approach as a robust unbiased tool for revealing new cortical specializations.

Supported by the Benoziyo Center.
Methysergide decreases self-grooming in suckling and weanling rat pups

Hecht D. and Leshem $M$

Dept. of Psychology and Brain \& Behavior Center, University of Haifa

Self-grooming behavior develops rapidly in the rat pup between suckling and weaning. Here we examined the role of two neural systems believed to mediate suckling in the adult, oxytocin and methysergide. Wistar rat pups 18-days-of-age (sucklings) and 30 -days-of-age (weanlings) received IP injections of saline or $5 \mathrm{mg} / \mathrm{kg}$ methysergide, $10 \mathrm{~min}$ later they were injected intracerebroventricularly with saline or the oxytocin antagonist, ornithine vasotocin, $0.5 \mu \mathrm{g} / 1 \mu \mathrm{l}$. Ten minutes later the pups were put in a Y-shaped arena with their anesthetized dam in one part, a sibling in another, and their behavior monitored for four minutes. Self-grooming was one behavior found to increase greatly from suckling to weaning. At both ages, the oxytocin antagonist and methysergide reduced self-grooming, and their combined effect was not additive. While self-grooming is known to be oxytocin-mediated, to our knowledge, this is the first demonstration of suppression of spontaneous self-grooming by a serotonin antagonist, and the first in suckling pups. Our findings suggest that serotonin may be no less important than oxytocin in mediating self-grooming behavior. Serotonin's role in self-grooming may be related to its role in mediating oral behavior, nipple attachment and contact with the dam in the pup. As the opportunity for these behaviors wanes with weaning, it may be that self-grooming replaces them, thereby maintaining the essential role of physical contact in development.

Supported by a GIF grant to K. Broun, \& M. Leshem.

Differential effect of emotional and physical stress on cortical dendritic spine development and possible role of 5-HT1 A-receptor blockade

Helmeke C., Abraham A. and Braun K

Dept. of Zoology/Developmental Neurobiology, Otto von

Guericke University, 39008 Magdeburg, Germany

We compared the effects of emotional and physical stress on the development of dendritic spines in somato-sensory cortex (SS) and limbic anterior cingulate cortex (ACd) of the trumpet-tailed rat Octodon degus. An involvement of the serotonergic system, in particular its $5 \mathrm{HTl}_{\mathrm{A}}$-receptor, on these stress-induced synaptic changes was tested. The densities of apical and basal dendritic spines of layer IV/III pyramidal neurons in the ACd and in the SS of 21 days old pups were quantified in the following animal groups: 1) social controls, 2) parentally separated animals (emotional stress), 3) saline injected animals (physical stress) 4) emotional stress plus physical stress, 5) parentally separated animals, which received injections of $0.1 \mathrm{mg} / \mathrm{kg}$ or 6) $1 \mathrm{mg} / \mathrm{kg}$ ), respectively, of the 5-HTl antagonist Way-100,635 prior to parental separation. Emotional and physical stress did not alter synaptic development in the somato-sensory cortex. In contrasi, spine development in the limbic anterior cingulate cortex is modulated by both, emotional and physical stress, but in a different direction. Emotional stress induced a significantly higher spine density in the anterior cingulate cortex compared to the social control group. Physical stress combined with emotional stress induced higher spine densities compared to

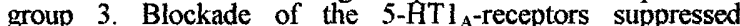
separation-induced spine elevation in the anterior cingulate cortex and decreases spine densities in the somato-sensory cortex. These results indicate that the synaptic development in the limbic (ACd) but not in the sensory (SS) cortex is sensitive towards stressful experience during early postnatal time windows, and that emotional or physical stress induce different synaptic changes in the ACd some of which appear to be mediated by $5-\mathrm{HT} 1_{\mathrm{A}}$-receptor activation.

Supported by grants from the VolkswagenStiftung and the German-Israeti Science Foundation

\section{High-level effects in the search for faces}

Hershler $\mathrm{O}$ and Hochstein $\mathrm{S}$

Dept. of Neurobiology, Institute of Life Sciences, Hebrew University, Jerusalem

Previously we showed that, contrary to older studies, human faces do pop out from a background of varied distractors. These results concur with the reverse hierarchy theory of vision (Ahissar \& Hochstein, 1997, 2002), which proposes that feature search reflects activity in higher cortical

areas as part of a rapid generalizing visual mode - vision at a glance. The present research focuses on when and how the face search mechanism is activated. We find that the pop out effect for human faces does not generalize to animal faces, which generated both longer search times 
and steeper and more varied search slopes. This suggests that the pop-out effect is specific for highly salient categories such as human faces. A second and third experiment examined which parts of the human face mediate the pop out effect. Results indicate that search is mediated by

the configuration of the whole face, rather than specific face parts. These and related findings indicate that search for a human face is indeed a high-level effect, confirming the claim of reverse hierarchy theory. Finally, we investigated the effect of subjective target salience on visual search performance. Supported by a "Center of Excellence" grant from the Israel Science Foundation and a grant from the US-Israel Binational Science Foundation (BSF)

\section{Copper binding to $\mathrm{PrP}^{\mathrm{C}}$ may inhibit prion disease} propagation

Hijazi N., Shaked Y., Rosenmann H., Ben-Hur T. and Gabizon $\mathbf{R}$.

Dept. Of Neurology; Hadassah University Hospital, Jerusalem

Although it has been well established that $\operatorname{PrP}^{\mathrm{C}}$, the normal isoform of $\mathrm{PrP}^{\mathrm{Sc}}$, is a copper binding protein, the role of this metal in the function of $\operatorname{PrP}^{\mathrm{C}}$ as well as in prion disease pathology remains unclear. Here we show that when scrapie infected neuroblastoma cells were cultured in the presence of copper, the accumulation of $\operatorname{Pr}^{\mathrm{Sc}}$ in these cells was markedly reduced. In addition, our results indicate that when normal neuroblastoma cells were cultured in the presence of copper ions, they could no longer bind and internalize $\operatorname{Pr} \mathrm{P}^{s e}$. In another set of experiments, copper was added to the drinking water of normal and scrapie infected hamsters. Our results show that administration of copper to normal hamsters induced cerebellar $\mathrm{Pr}^{\mathrm{C}}$ accumulation. Most important, a significant delay in prion disease onset was observed when scrapie infected hamsters were treated with copper. As shown before for neuroblastoma cells, also in-vivo most of the copper induced accumulation of $\operatorname{Pr}^{\mathrm{C}}$ was intracellular. We hypothesized that $\operatorname{PrP}^{\mathrm{C}}$ internalization by copper may hinder $\mathrm{Pr}^{\mathrm{sc}}$ interaction with this molecule, and thereby affect prion disease propagation.

\section{Hemispheric specialization and interhemispheric}

interaction in schizophrenia

Hirschmann $\mathrm{S}^{1,2}$, Lamschtein $\mathrm{C}^{1},{ }^{1}$ BazBarChani $\mathrm{M}^{1}$, Barnea

$A^{3}$, Zaidel $E^{4}$

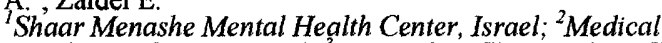

Faculty, Technion, Israel, ${ }^{3}$ Bio-Keshev Clinic, Kibutz Givat

Chaim Ichud, Israel; ${ }^{4}$ Dept. of Psychology, UCLA, USA.

We used a hemifield tachistoscopic lexical decision task in Hebrew, with lateralized targets and distractors, to measure (1) hemispheric specialization (right visual field advantage), (2) psycholinguistic strategy (wordness advantage), (3) interhemispheric transfer (lexicality priming, i.e., better decision when the target and distractors are the same response category, namely, both words or both nonwords), and (4) spontaneous error adjustment (slowing down and becoming more accurate following errors) in 23 acute patients suffering from schizophrenia (Sz) with predominant positive or negative symptoms. Twenty eight normal adults served as controls. Contrary to previous claims, relative to control, Hebrew speaking Sz (reading from right to left) showed a larger right-sided visual field a-symmetry in accuracy $(F(1,47)=6.53$, $p=014)$ and in sensitivity $(F(1,47)=4.75, p=.034)$, signaling greater left hemisphere specialization for word recognition, (2) a greater disadvantage in non-word recognition in both accuracy $(F(1,47)=7.0, p=.01)$ and in latency $(F(1,47)=7.64$, $\mathrm{p}=.0081$ ), (3) increased left hemisphere-to-right hemisphere transfer (accuracy: $F(1,47)=6.24, p=016$ ), and (4) a similar pattern of slowing down and a similar inability to improve accuracy following errors. These effects were largely due to a selective deficit of $\mathrm{Sz}$ in the left visual hemifield/right hemisphere.

The right visual field advantage, lexicality priming, and error adjustment measures in latency and accuracy were tested for correlation with positive and negative symptoms. Negative symptoms were significantly and negatively correlated with the right visual field advantage in accuracy $(\mathrm{r}=-.525, \mathrm{p}<.01)-$ implying that higher negative symptoms are associated with weaker left hemisphere dominance. At the same time, Positive symptoms were significantly and negatively correlated with lexicality priming in latency $(r=.500, p<.01)$, indicating that higher positive symptoms are associated with weaker (inhibitory) interhemispheric communication.

These results suggest that the negative symptoms reflect deficient hemispheric specialization, whereas positive symptoms reflect deficient interhemispheric communication
A comparative genomic study reveals an extreme level of conservation of genes involve in synaptic function between the fruit fly and honey bee

Inberg $A$. and Linial M.

Dept. of Biological Sciences, Institute of Life sciences, The

Hebrew University, Jerusalem

The Phylum Arthropoda that includes all insects is the most morphologically diverse animal group on the planet. Since the appearance of insects they dominate most ecological habitats. The insects and especially the Drosophila had been used as one of the most successful genetic model to study neuronal development, differentiation and simple behavior. Following sequencing the Drosophila (and later the Anopheles) genomes, a more remote organism was selected to cover the divergence of this phylum. The $135 \mathrm{Mbase}$ genome of the honeybee (Apis mellifera) is being sequenced. Honeybees have a complex social behavior that was extensively studied. It is hypothesized that the social behavior will be reflected by the complexity of genes and their variants. With the availability of complete unassembled Apis mellifera DNA fragments and partial cDNA collection, we search for a direct homology to Drosophila genes. We selected over 150 neuronal genes that are known to participate in shaping the synapse, in learning, and memory, in secretion and endocytosis and in membrane physiology as our test set. Among these genes are those associated with SNAREs, synaptic vesicle, post-synaptic PDZ, ion channels and endocytosis.

We show that all tested 150 genes are conserved between the fly and the honeybee. Most notably is the extremely high degree of conservation ( $>95 \%$ identical aa) among the various Shaker type potassium channels between the two organisms. The homology with other synaptic proteins is also extremely high (ranging from $80-95 \%$ aa identity). Mammalian synaptic vesicle proteins such as synaptophysin that are not present in Drosophila were also missing in Apis genome. We aimed to use the comparative genome approach to search for the diversity in protein families between fly, bee, worm and mouse. Such a comparative research will provide us with new insights into the genetic makeup that underlying synaptic function and complex behavior.

\section{Differential activation of hippocampus and amygdala} under controllable or uncontrollable stress

Ilin Y. and Richter-Levin G.

Dept. of Psychology; Brain and Behavior Research Center, Haifa University.

Stress has multiple modes of influence on learning and memory, improving or impairing it under different conditions The Hippocampus is held accountable for explicit memory The amygdale is critically involved in mediating stress-related effects on behavior.

The MAPKs (ERK) are recruited when the brain undergoes synaptic plasticity and remodeling (e.g., during induction of long-term potentiation, learning and memory consolidation).

It has been suggested that the amygdale involvement will be greater under condition of uncontrollable VS controllable stress. To test this possibility four groups of rats were tested: 1 . Good Learners (controllable situation) - subjected to avoidance learning protocol in the shuttle box. 2 . Bad learners (controllable situation) - subjected to avoidance learning protocol in the shuttle box, but have not acquired the task well. 3. Stressed rats (uncontrollable situation) - subjected to parameters of good learners without the possibility to avoidance learning. 4. Nałve rats. Groups 1,2 , and 3 were trained in the Shuttle Box, and 30 minutes after the test session, the Hippocampus, the Amygdala and the Prefrontal Cortex were collected for analysis of ERK activation during Western Blotting procedure.

Differences were found in freezing responses $(3 \mathrm{~min}$ freezing test in shuttle box) between Good learners on one side and bad learners and Stressed rats on the other side, indicating that indeed the context of the shuttle box was most aversive to the Stressed and bad learners. The main finding was a differential activation of ERK in the amygdala following an exposure to uncontrollable condition (stressed rats) compared to all other conditions.

The results confirm our hypothesis that the involvement of the amygdala is increased when being exposed to an uncontrollable condition.

Supported by The Israel Foundation - The Charles H. Revson Foundation (no. 582/00-1 to G.R-L). 
The anti-inflammatory and cholinesterase inhibitor bifunctional compound IBU-PO protects culture neurons from $A B$-induced cytotoxicity by acting on Wnt cell signaling components

Inestrosa N.C. . Godoy J.A. ${ }^{1}$, Farias G.C. ${ }^{1}$ Avila $]^{2}$ Fernández $\mathrm{F}^{2}$, Vásquez M.C. ${ }^{1}$, Muñoz F.J. ${ }^{3}$, Adani $\mathrm{R}^{4}$ Meshulam $\mathrm{H}^{4}$ and Amitai $\mathrm{G}$.

${ }^{I}$ P.U. Católica de Chile, Santiago, Chile; ${ }^{2}$ Centro de Biol. Molecular "Severo Ochoa", U. Autónoma de Madrid, Spain; ${ }^{3}$ Ciències Exper. i de la Salut, U. Pompeu Fabra, Barcelona, Spain. ${ }^{4}$ Pharmacology, IIBR, Ness Ziona, Israel

The current therapy of Alzheimer's disease (AD) is based on cholinesterase inhibitors (ChEI). It was noted that certain non-steroidal anti-inflammatory drugs (NSAIDs) retard or avoid the onset of AD. Therefore, a series of novel bifunctional compounds have been synthesized based on the molecular combination of ChEIs with NSAIDs. The bifunctional compound Ibuprofen-Octyl-Pyridostigmine (IBU-PO) displays neuroprotective activity in CNS inflammation, traumatic brain injury, experimental autoimmune encephalomyelitis and brain hypoxia in mice. The racemic $( \pm) I B U-P O$ and the enantiomer $(+)$ IBU-PO were tested for their neuroprotective activity against amyloid $\beta$-peptide $(A \beta)$-mediated cytotoxicity in rat hippocampal neurons ( $\mathrm{RHN}$ ) and mouse cortical neurons (MCN) in vitro. IBU-PO increased dose-dependently at sub-micromolar levels $(0.01-1 \mu \mathrm{M})$ the viability of RHN challenged by $A \beta(5 \mu \square \square$. The mechanism of action is related to two key members of the Wnt cell signaling: $\beta$-catenin and GSK-3 $\beta$. The levels of $\beta$-catenin in RHN reduced by $A \beta$ were recovered completely by IBU-PO at $0.1-1 \mu \mathrm{M}$. Inactivation of GSK-3 $\beta$ was studied by phosphorylation of Ser-9-GSK-3 $\beta$. ( \pm IBU-PO caused a dose-dependent increase in the phosphorylation of Ser-9-GSK-3 $\beta$ in MCN from wild type and GSK-3 $\beta$ overexpressing mice. The levels of cytoplasmic $\beta$-catenin were stabilized by $( \pm)$ IBU-PO in MCN from both types of mice. $(+)$ IBU-PO, caused an increase in the phosphorylation of Ser-9-GSK-3 $\beta$ in neurons from both types of mice but lower than with $( \pm)$ IBU-PO. Thus, stabilization of cytoplasmic $\beta$-catenin and inhibition of GSK-3 $\beta$ were displayed in RHN and MCN. Moreover, IBU-PO enhanced the non-amyloidogenic APP cleavage by causing an increase in the secreted APP level as well as a decrease in $A \beta_{1-40}$ in RHN.

Amelioration of experimental autoimmune encephalomyelitis by recombinant human

alpha-fetoprotein
Irony Tur-Sinai M. ${ }^{1}$, Grigoriadis $\mathrm{N}^{2}$ and Brenner T. ${ }^{1}$. 'Laboratory of Neuroimmunology, Dept. of Neurology, Hadassah Hebrew University Medical Center, Jerusalem ${ }^{2}$ Aristotle University, Thessaloniki Greece.

Alpha-fetoprotein (AFP) is an immunomodulating embryo-specific glycoprotein produced by the yolk sac and fetal liver. AFP concentrations in maternal human serum increase gradually in the second and third trimesters of pregnancy and drop rapidly at the time of delivery. Clinical remissions during the second half of pregnancy, as well as a tendency to postpartum relapses, have been described in several autoimmune diseases such as multiple sclerosis, myasthenia gravis, rheumatoid arthritis and thyroiditis, and are attributed to the immunosuppressive effect of AFP. Our previous studies showed that human AFP, isolated from maternal or fetal serum, and recombinant human AFP (rhAFP), cloned and expressed in E.coli, suppress experimental autoimmune encephalomyelitis (EAE) and experimental autoimmune myasthenia gravis (EAMG) Recently, following the development of a novel technology based on the secretion of rhAFP in the milk of transgenic goats, we tested this preparation in the EAE model. The goat-derived rhAFP preparations caused marked suppression of EAE. Under AFP treatment fewer animals developed EAE and none of them died. CNS tissue from AFP-treated mice showed a pronounced reduction in the degree of inflammation, axonal loss and axonal injury in all the AFP-treated groups, as opposed to the placebo-treated mice. CNS damage was prevented or limited in the brain and spinal cord meninges and parenchyma, and was correlated with disease severity and incidence Also treatment of EAMG rats with rhAFP conferred a beneficial effect, particularly during the chronic phase of the disease. Our observations further support the role of AFP in the amelioration of experimental autoimmune diseases and other autoimmune disorders.
Epileptiform activity and cortical spreading depression in acute and chronic models of epilepsy

Ivens $S^{1}{ }^{1}$, Heinemann $U^{1}$ and Friedman $A^{1}, 2$

'Institute of Physiology ${ }^{2}$ Exp. Neurology, Charit, Humboldt

University, Berlin, Germany and ${ }^{3}$ Zlotowski Center of

Neuroscience, Ben Gurion University, Beersheva, Israel

Cortical spreading depression (CSD) is a depolarization wave propagating at a rate of $\sim 3 \mathrm{~mm} / \mathrm{min}$ in the cerebral cortex. The phenomenon is triggered by electrical, mechanical or toxic factors and is considered the best known model for migraine aura. CSD was also associated with epilepsy as both migraine and epilepsy are disorders characterized by transient paroxysmal propagating cortical dysfunction and in a number of syndromes, migraine and epilepsy are related. To search for the sensitivity of different cortical regions to initiation and propagation of epileptiform activity and CSD initiation, we used coronal brain slices maintained in-vitro. Spontaneous or stimulus-evoked epileptiform activity or CSD were evoked in the presence of high- $\mathrm{K}^{+}(8-15 \mathrm{mM})$, Low $\mathrm{Mg}^{++}(0-1 \mathrm{mM})$, 4-aminopyridine $(10 \mathrm{mM})$ or bicuculin $(5 \mathrm{mM})$ in slices from control rats or from rats with a chronic epileptic focus following BBB disruption. Recordings were made using 2-4 $\mathrm{K}^{+}$sensitive microelectrodes. Velocity of the propagating epileptiform activity and CSD were measured over different cortical regions (motor, somatosensory-trunk and whisker region and auditory cortex). Our data shows that under different models of increased excitability, ictal-like events as well as CSD generation is more readily triggered in the chronic epileptic cortex. Moreover, ictal events and CSds had longer duration and slower recovery in the epileptic cortex compared to non-treated regions. As glutamate receptor antagonists blocked and GABA-antagonists enhanced abnormal hypersynchronous activity, we propose that focal cortical reorganization with abnormally increased excitatory drive may prone distinct cortical regions to both $\mathrm{CSD}$ and focal epileptiform activity.

\section{Therole of $\mathrm{GABA}_{\mathrm{A}}$ receptor subunits in the development} of affective disorders

Jacobson $\mathrm{S}$. and Richter-Levin $\mathrm{G}$.

Dept. of Psychology, University of Haifa, Center of Brain and Behavior

A new animal model of affective disorders was developed indicating that exposure to both juvenile (26-28 days) and adult (60 days) stress increases anxiety levels. Profound evidence indicates that $\mathrm{GABA}_{\mathrm{A}} \mathrm{Rs}$ are important in the control of the physiological response to stress and anxiety. There are at least 19 related $G_{A B A} R$ subunits the $G A B A_{A} R$ are best distinguished by the type of $\alpha$ subunit. The localization and constitution of $\mathrm{GABA}_{\mathrm{A}} \mathrm{R} \alpha$ subunits varies during embryonic and postnatal ontogeny. In embryonic and early postnatal period of normal rats the expression of $\alpha_{2}, \alpha_{3}$ and $\alpha_{5}$ subunits is high while $\alpha_{1}$ subunit expression is low. In later developmental stages there is a rapid increase in the proportion of $\alpha_{1}$ subunit whereas $\alpha_{2}, \alpha_{3}$ and $\alpha_{5}$ subunits disappear from numerous areas soon after the $\alpha_{1}$ subunit appears. In the present study we began to assess whether in affective disorders emotional abilities are impaired due to changes occurring in the GABAergic system. We have utilized the juveniletadult exposure to stress model to test potential alterations in the expression of $\mathrm{GABA}_{\mathrm{A}} \mathrm{R}$ subunits in the amygdala and hippocampus. We compared two groups: 1) Juvenile+adult exposure to stress group- rats were exposed to both platform stress paradigm and acute swim stress paradigm. 2) Control group- rats were not exposed to stress. Following the adult exposure to stress we conducted anxiety tests and collected brain tissues. We used western blotting technique for the detection of specific $\mathrm{GABA}_{\mathrm{A}} \mathrm{R} \alpha$ subunits $\left(\alpha_{1}\right.$ and $\left.\alpha_{5}\right)$ Following exposure to stress, we found abnormal patterns of $\mathrm{GABA}_{\mathrm{A}} \mathrm{R} \quad \alpha_{1}$ and $\alpha_{5}$ subunit expression. These patterns suggest that there is an incomplete switch from the $G A B A_{A} R$ $\alpha$ subunit highly expressed at the time of birth $\left(\alpha_{5}\right)$ to the predominant subunit of the adult brain $\left(\alpha_{1}\right)$. In the stress group, the functional consequences might be a faulty maturation of the inhibitory GABAergic system that may lead to increased anxiety levels.

\section{Association between BMP signaling, the cell cycle and}

\section{neural crest delamination}

Kalcheim C. Stanleigh J. and Burstyn-Cohen T.

Dept. of Anatomy and Cell Biology Hebrew University Hadassah Medical School. Jenusalem 91120

We have previously found that delamination of premigratory neural crest $(\mathrm{NC})$ cells from the dorsal neural tube depends both upon BMP/noggin signaling and a successful transition from Gl to the S phase of the cell cycle. We then investigated the hypothesis that these two mechanisms are hierarchiclly related. Inhibiting BMP in the dorsal tube by overexpression of 
noggin reduced the proportion of cells entering S-phase as well as expression of cyclin DI mRNA. This was associated with loss of transcripts encoding Wntl, suggesting that Wntl acts downstream of BMP. To investigate whether Wnt signaling links BMP activity to the cell cycle and in turn to NC delamination, we overexpressed by electroporation a mutant form of dishevelled (Xdd1) that abrogates both canonical and non-canonical Wnt activities. Xddl inhibited both the entry of nuclei into S-phase and the delamination of NC cells Altogether, we suggest that the activity of BMP in the neural tube is mediated by Wnt signaling which controls cell cycle progression and consequently the generation of NC cell movement.

Distribution and role of proteasome in the transformation of an axon into a growth conc after axotomy

Kamber D. and Spira M.E.

Dept. of Neurobiology, Life Science Instttute. The Hebrew

University of Jerusalem.

Recent studies indicate that protcasumes play central rolcs in different forms of neuroplasticity including turning and retraction of growth cones (GC) in response to signaling molecules (Douglas \& Holt, 2001, Neuron 32) and in establishment of short and long term synaptic plasticity (Zhao et al., 2003, Curr. Biol. 13). These reports as well as carlier findings by our laboratory (Gitler \& Spira, 1998, Neuron 20) raise the possibility that proteasomes participate in other forms of neuroplasticity such as neuronal regeneration after trauma.

In the present study we use cultured Aplysia neurons and on line confocal imaging to examine the distribution and role of proteasomes in the transformation of a differentiated axon into a GC after axotomy.

To image proteasome activity we used the membrane-permeable proteasome substrate succinyl-Leu-Leu-Val-Tyr-7-amido-4-trifluoromethyl

coumarin (suc-LLVY-AFC). Unlike suc-LLVY-AFC its cleavage product AFC is highly fluorescent. Baseline proteolytic activity was imaged in intact neurons, and was found to reach a steady state (SS) level $\sim 40 \mathrm{~min}$ after addition of the indicator to the bathing solution. Axonal transection leads to elevation of the product's fluorescence in the most distal zone (DZ) of the transected axon, reaching a new SS level that remains high for hours.

Immunolabeling with anti prosomes p25K (ICN Pharmaceuticals, Inc) revealed that axotomy leads to proteasomes accumulation at the $\mathrm{D} Z$ and to its translocation to the membrane and submembrane domains of the GC's lamellipodium. Thus the distribution of the proteasome and the proteolytic product colocalizes within the GC

To examine whether proteasomes activity play any role in the transformation of an axon into a $G C$, we bathed the intact neuron in $10 \mathrm{mM}$ clasto-Lactacystin b-lactone, a membrane-permeable proteasome inhibitor. Under these conditions the protcolysis of Suc-LLVY-AFC and the formation of a GC's lamellipodium after axotomy were greatly inhibited.

Supported by the BSF

Unexpected enhancement of LTP in brain slices by hydrogen peroxide

Kamsler $A$ and Segal $M$

Dept. of Neurobiology, Weizmann Institute of Science, Rehovot Hydrogen peroxide $\left(\mathrm{H}_{2} \mathrm{O}_{2}\right)$, a reactive oxygen species, is assumed to have a detrimental effect on neuronal plasticity. Indeed, $\mathrm{H}_{2} \mathrm{O}_{2}$ suppresses LTP in hippocampal slices of normal rats and wild-type (wt) mice. Transgenic mice overexpressing SODl (tg-SOD), which maintain high ambient $\mathrm{H}_{2} \mathrm{O}_{2}$, have also been shown to be impaired in their ability to express hippocampal LTP. Paradoxically, $\mathrm{H}_{2} \mathrm{O}_{2}$ at a concentration $(50 \mu \mathrm{M})$ that blocks LT'P in wt mice, actually enhanced LTP in slices of 2-month-old tg-SOD mice. $\mathrm{H}_{2} \mathrm{O}_{2}$ dependent LTP in tg-SOD was blocked by the protein phosphatase calcineurin inhibitor FK-506, but not by rapamycin, an FKBP12 inhibitor or by $\mathrm{H7}$ a serine-kinase inhibitor. Interestingly, wt and tg-SOD mice expressed similar levels of the antioxidant enzyme catalase and similar activity of glutathione peroxidase. An opposite situation was found in 2 yr old mice. Aged wt mice were impaired in LTP in a manner that could be reversed by addition of $\mathrm{H}_{2} \mathrm{O}_{2}$. Surprisingly, aged tg-SOD mice exhibited larger LTP than that found in wt mice but this was now reduced by $50 \mathrm{mM} \mathrm{H}_{2} \mathrm{O}_{2}$. Both young tg-SOD and aged control mice displayed altered protein phosphatase activity compared to that of young controls, moreover, FK506 inhibited LTP in old tg-SOD as well as in old wt mice treated with $\mathrm{H}_{2} \mathrm{O}_{2}$. These data promote a role for $\mathrm{H}_{2} \mathrm{O}_{2}$ in the regulation of LTP, and proposes that it is mediated by the protein phosphatase calcineurin.
Extracellular regulated kinase I/II (ERKI/II) as a coincident detector of neuromodulation and fast neurotransmission

Kaphzan H. Belelovsky K. and Rosenblum K. Center for Brain and Behaviour, Faculty of Science and Science Education, University of Haifa 31905

In order for a particular neural representation to become consolidated for longer periods, long lasting changes in the neural comectivity should take place, as well as other intrinsic neural changes. These changes depend upon molecular processes such as signal transduction, transcription and protein synthesis.

We hypothesize that in order for an afferent ionotropic "fast" exprerience to be consolidated as a long term memory, it should come in convergence with another metabotropic "slow" modulatory input This modulatory-metabotropic input ignite a molceslar process, that when converged with the "fast" ionouropic inpul, it promotes the possibility for that specific "fast" input to be consolidated into a long term memory. The molccular convergence sites of the two inputs might serve as their coincident detectors, and act as a molecular "decision" point for consolidating current experience to a long term memory.

One such molecular converging site, which is activated by both types of synaptic activities (i.e. "fast" and "slow") is the ERK molecule. In addition, ERKI/II was found to be necessary for memory/synaptic plasticity consolidation in different brain areas.

In order to test the hypothesis that ERKI/II serve as a coincident detector of fast and slow neurotransmission we developed a system that utilized pharmacology with brain slices. The system enables us to have a full and accurate control over the pharmacological manipulations in means of time scales and doses, while giving us a close biological erivironment as possible to the functioning mature brain in vivo.

Preliminary results with hippocampal slices have shown that $100 \mu \mathrm{M}$ NMDA (as a "fast" neurotransmitter) induced an incrcase in ERKI/II activation within 5 minutes. ERKI/II activation returned to base line levels within 15 minutes presumably as a result of significant activation of ERKI/I specitic phosphatase/s. We are currently analyzing the effect of neuromodulators (e.g. dopamine) on ERKI/II activation with and without the NMDA input.

Urine from scrapie infected hamsters comprises low levels of prion infectivity

Kariv-Inbal Z. ${ }^{1}$, Shaked G.M. ${ }^{1}$, Grigoriadis N. ${ }^{2}$ and Gabizon

${ }^{1}$ Dept of Neurology; Hadassah University Hospital, Jerusalem Israel, 'Dept. of Neurology AHEPA, Aristotale University Hospital, Thessaloniki Greece

The question of whether prion diseases can be transmitted by body fluids has important epidemiological and economical implications. The possibility that prion-infected urine is infectious has also environmental implications, such as the transmition of CWD and scrapie between animals in the field. However, no infectivity has been reported as for today in prion-infected urine

Following our findings suggesting that a protease resistant isoform of PrP may be present in prion-infected urine, we tested the urine infectivity of scrapie sick hamsters by infecting normal hamsters with enriched urine samples by several routes of infection. Following prolonged incubation times, two animals, each one in a different group succumbed to prion disease while most of the others in the same groups present symptoms of sub-clinical prion disease, including low levels of $\mathrm{PrP}^{\mathrm{Sc}}$ in their brains and extensive glyosis. Our results therefore suggest that prion-infected urine comprises low levels of infectivity. We also propose that in general, when low levels of prion infectivity are expected, it is important to test the presence of sub-clinical signs in the infected animals

\section{Functional properties of anterior pituitary cells in} hypothalamo-pituitary slices of the teleost fish tilapia Kasher C., Fleidervish I.A., Zakai H., Gutnick M.J., and Levavi-Sivan B.

Koret School of Veterinary Medicine \& Dept. of Animal

Sciences, The Hebrew University of Jerusalem, Rehovot 76100

The anterior pituitary of the teleost fish contains a variety of endocrine cells, which, under direct control from hypoihalamus, release somatotrophic and gonadotrophic hormoncs and thereby play a major role in fish reproduction social behavior and growth. Although the edocrinological properties of these cells have been extensively studied, little is knowi about their underlying biophysical properties. Here, we have studied the electrophysiological properties of these cells 
using a novel in-vitro slice preparation that leaves much of the hypothalamo-pituitary circuitry intact.

In whole cell current-clamp recordings the cells had membrane potentials between -65 and $-75 \mathrm{mV}$, and a very high input resistance. In response to depolarizing current pulses they fired single, brief, fast-rising action potentials which were followed by pronounced afterhyperpelarization. Voltage-clamp experiments revealed the $\mathrm{Na}^{2}, \mathrm{Ca}^{2+}$, and $\mathrm{K}^{+}$ conductances that underlie these activities. In addition, the cells were found to possess a strongly outwardly rectifying cation-nonspecific furrent which was resistant to classical
blockers of $\mathrm{Na}^{+}, \mathrm{Ca}^{++}$and $\mathrm{K}^{+}$channels. The kinetic properties of this current were similar to those ascribed in other neuronal and non-neuronal systems to currents mediated by TRP channels. We therefore performed Western blots with TRPC channel-specific antibodies to determine whether TRP channels are indeed present in the membranes of fish anterior pituitary cells. We detected high levels of TRPC4 and 6 in the pituitary homogenate, while TRPCl, 3 and 5 were not present. When Lucifer Yellow was introduced into a single cell in order to mark it, the fluorescent dye quickly stained neighboring cells as well. Since Lucifer Yellow freely passes through gap junctions, we recorded simultaneously from pairs of adjacent pituitary cells and found that they are strongly electrotonically coupled. These data lead us to hypothesize that the electrical activities of anterior pituitary cells in tilapia are synchronized by coupling through gap junctions, and that regulation of this coupling may play a critical role in determining complex patterns of pituitary hormone secretion.

Effect of maternal exposure to hypoxic episode on postnatal development; possible protective effect by maternal administration of magnesium sulfate Kashtutsky $1 ., 4,5$, Huleihel $M^{2,4}$, Hallak M. ${ }^{3,4}$, Sorokin $Y^{6}$, Galili $\mathrm{M}^{4}$ and Golan $\mathrm{H}^{1}$.

Dept. of Developmental Genetics', Dept. of Immunology ${ }^{2}$, Dept. of Obstet. \& Gynecol. ${ }^{3}$, Faculty of Health Sciences Zlotowski ctr. for Neuroscience, $B G U$ Beer-Sheva, Israel ${ }^{6}$ Dept. of Obs. \& Gyn. Hutzel Hospital. Detroit, Michigan, USA

Prenatal hypoxic-ischemic brain injury is believed to cause permanent neurological deficits in neonates. It was suggested that the damage to fetus brain involved glutamate toxicity mediated by the NMDA receptor. We examined the possible protective effect of magnesium sulfate $(\mathrm{Mg})$ against glutamate toxicity. Since NMDA receptor is involved in accurate neurogenesis, $\mathrm{Mg}$ may impair development. Here, we tested the effect accreted by maternal hypoxia and possible protective effect of magnesium sulfate treatment in a mouse model. Pregnant mouse at gestation day 17 were exposed to: 1. Hypoxia, following pretreatment with saline $(\mathrm{S}+\mathrm{H}), 2$. Hypoxia, following pretreatment with $\mathrm{Mg}(\mathrm{Mg}+\mathrm{H}), 3$. Control group, air breathing following saline injection (S+A) and 4. Air breathing following $\mathrm{Mg}$ injection $(\mathrm{Mg}+\mathrm{A})$. During the first month, newborns were tested for the development of motor reflexes and coordination. The development of $\mathrm{S}+\mathrm{H}$ newborns was slow in the righting reflex, rotarod and locomotion on inclining slope. $\mathrm{Mg}$ pretreatment improved the performance of newborn in all reflexes, resulting in similar score as the control group. Thus, hypoxia delay the development of motor function and pretreatment with $\mathrm{Mg}$ compensate for the impairment caused by hypoxia.

At the age of 3 months mice of all treatment exhibited reduced number of moves within 5 min $(25,6,24.7,24.5)$ in the open field as compared to the control (31.7). In all treatment groups $33-44 \%$ of mouse develop ataxia as indicated by the hind-paw footprint, compared to $8 \%$ in the control group, stride size was significantly shorter in the $\mathrm{Mg}+\mathrm{A}$ group, as compared to the control. Motor impairment was further addressed by wire hanging test and vertical pole. All control mice hang to the wire for $60 \mathrm{sec}$ while in both $\mathrm{S}+\mathrm{H}$ and $\mathrm{Mg}+\mathrm{A}$ groups, $25 \%$ and $27 \%$ of mice fail to hold, and only $12.5 \%$ of the $\mathrm{Mg}+\mathrm{H}$ group In a vertical pole $18 \mathrm{~mm}$ diameter, $50 \%$ of $\mathrm{S}+\mathrm{A}$ and $\mathrm{Mg}+\mathrm{A}$ mice hold for $60 \mathrm{sec}$, only $25 \%$ of $\mathrm{S}+\mathrm{H}$ and $38 \%$ of $\mathrm{Mg}+\mathrm{H}$ could hold. In summary, hypoxia delay the development of motor function and impair adult mice motor performance, pretreatment with $\mathrm{Mg}$ partially compensate for the impairment caused by hypoxia.
Low molecular weight heparin but not aspirin ameliorates the behavioral hyperactivity in an experimental model of antiphospholipid syndrome

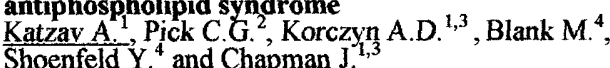

Depts. of ${ }^{l}$ Physiology and Pharmacology, ${ }^{2}$ Anatomy, and

${ }^{3}$ Neurology, Sackler Faculty of Medicine, Tel Aviv University, Tel Aviv 69978, and ${ }^{4}$ Dept . of Medicine $B$ and Research Unit of Autoimmune Diseases, Sheba Medical Center, Tel

Hashomer 52621

Background: The antiphospholipid syndrome (APS), defined by the occurrence of recurrent thromboembolic events (venous or arterial), repeated spontaneous abortions, thrombocytopenia and the presence of elevated titers of circulating antiphospholipid antibodies (aPL) is associated with neurological manifestations. Animals immunized with $b_{2}$-glycoprotein I $\left(b_{2}-G P I\right)$, a serum protein which is essential for the binding of pathogenic aPL, develop all the systemic signs of APS as well as behavioral hyperactivity. Objective: To investigate the pathogenesis of CNS involvement in experimental APS by modulation of coagulation by low-molecular-weight heparin (LMWH, Clexane) or aspirin in this model. Methods: Female $\mathrm{Balb} / \mathrm{C}$ mice were immunized with $b_{2}-G P I$ in complete Freund's adjuvant (CFA) or with CFA alone. Treatment was started 6 weeks after immunization and 3 months later the mice behavior was tested in the staircase test. Results: Immunization of naive mice with $b_{2}$-GPI resulted in elevated levels of circulating anti-negatively charged phospholipids, and anti- $b_{2}$-GPI antibodies. The APS mice exhibited hyperactive behavior compared to controls as reflected by higher rate of stair climbing ( $<<0.008$ by t-test) on the apparatus. Aspirin treatment $(30 \mathrm{mg} / \mathrm{mouse})$ had no effect on the hyperactive behavior of APS mice $(p<0.04$ by t-test). In contrast, LMWH treatment ( $25 \mathrm{mg} /$ mouse) normalized APS mice behavior. Conclusions: The beneficial effect of LMWH on the hyperactivity of APS mice implicates vascular pathology and coagulation factors in causing CNS disease. This effect as well as the lack of efficacy of aspirin may be relevant to the treatment of APS in humans.

A possible function of no in learning that food is inedible in Aplysia: NO signals that animals have attempted to eat Katzoff A. Miller N., Hurwitz I., Susswein Y.Z. and Susswein A.J.

Faculty of Life Sciences, Bar Ilan University, Ramat Gan

Aplysia feeding is modified by associative learning. Food contacts the lips, enters the mouth, and elicits attempts to swallow. If food cannot be swallowed, Aplysia responses are weakened. Thus, reinforcement arises from success or failure to swallow. However, a condition for learning is that food must enter the mouth: food stimulating the lips, without entering the mouth, causes no learning. Learning is followed by short and long term memory, which are blocked by treatment with antagonists of NO during training. Treating with the NO donor SNAP restores the ability of training to produce memory.

Cerebral ganglion neuron $\mathrm{C} 2$ synthesizes and releases $\mathrm{NO}$, which excites the serotonergic modulatory metacerebral cell (MCC). $\mathrm{C} 2$ is a primary mechano-afferent innervating the peri-oral zone. Entry of food into the mouth, and pulling at the food in attempts to swallow, activate $\mathrm{C} 2$, and therebv excite the MCC. We examined the hypothesis that NO in learning signals food entry into the mouth, I.e., reports that the operant has occurred. When lips were stimulated with food, but food did not enter the mouth, no short- or long- term memory occurred. However treating animals with the NO donor SNAP prior to lip stimulation without food entry led to both shortand long-term memory. Thus, NO treatment mimicked the effect of food entery, and converted an ineffective procedure in producing memory into an effective procedure

To examine whether the MCC is activated by SNAP that support memory formation, extracellular recordings from the MCC were performed with and without SNAP, in response to applications of carbamyl-choline $(\mathrm{CCh})$, which elicits fictive buccal motor programs. SNAP elicited activity in the MCC and also amplified the response to $\mathrm{CCh}$. The increase occurred with a delay of $\sim 10 \mathrm{~min}$ and was maintained for $15-30 \mathrm{~min}$ after washing out the $\mathrm{CCh}$. These data support the hypothesis that NO signals food entry into the mouth and thereby causes an increased firing of MCC. This increase is essential for learning. 
Activity and plasticity in the dentate gyrus and the amygdala following controllable vs. uncontrollable stress
Kavushansky A. and Richter-Levin G.

Dept. of Psychology, University of Haifa, Haifa 31905

We studied the effects of controllable versus uncontrollable stress on synaptic plasticity in the hippocampus and in the amygdala. The hippocampus is a brain region involved in the processing of the cognitive aspects of emotional experiences. The amygdala is related to variety of emotional functions. We used the rat model and the electrophysiological procedure of long-term potentiation (LTP) induction in both structures simultaneously, stimulating the entorhinal cortex (EC). EC is located at a nodal point between the hippocampus and the amygdala and has reciprocal projections to both brain areas. The najve group was left undisturbed until the recording. Rats of the controllable stress group ("platform") were trained in the Morris water maze to locate the hidden underwater platform and so to escape the cold water $(19-21 \div \mathrm{C})$, immediately before the recording. The uncontrollable stress group ("no platform") underwent the same procedure without the platform. Rats were anesthetized, and after the recording of baseline field potentials in the dentate gyrus and in the basolateral amygdaloid nucleus, theta-burst stimulation was given to the $\mathrm{EC}$, and recording proceeded for $1 \mathrm{~h}$. Similar to previous results from our lab, stimulation of the EC induced LTP in both the hippocampus and the amygdala. The stress procedures did not significantly affect the LTP in the dentate gyrus. The baseline in the amygdala of the "no platform" group was enhanced compared to the najve group, suggesting uncontrollable stress-induced plasticity in the amygdala. Future research will assess plasticity changes in the $\mathrm{CAl}$ area of the hippocampus following controllable and uncontrollable stress

Real-time detection of nitric oxide producing interneurons

in cortical slices by diaminotriazolofluorescein

Timbi S. ${ }^{T}$ Dept of Physiology, Ben-Gurion University, Beer-Sheva and ${ }^{2}$ Dept of Physiology and Biophysics, Technion, Haifa.

Various anatomical methods reveal that only a subset of GABAergic neurons expresses the enzyme nitric-oxide synthase in cortical neuronal circuits. However, the production of nitric oxide by cortical neurons has never been explored We used the fluorescent indicator for nitric oxide (NO), diaminotriazolofluorescein (DAF-2T) to reveal NO-producing neurons in live neocortical slices.

Acute brain slices were incubated for 15 minutes in a ringer solution containing $2 \mu \mathrm{M}$ of the DAF-2T precursor DAF-2 $\mathrm{DA}$, and then transferred to a slice bath mounted on a fluorescent microscope equipped with IR/DIC optics, where we attempted recording from stained cells. Some experiments were carried out using an upright confocal microscope. Intensely stained neuronal somata and proximal dendrites were visible after several minutes of excitation with $488 \mathrm{~nm}$ wavelength. In addition, there was a diffuse punctate staining in all layers, with particularly high density in laver I. Cells stained with DAF-2T were readily identified under IR/DIC. None of these neurons had pyramidal morphology, and most of them were slightly shrunken and apparently dead. Neighboring neurons could be patched and displayed normal physiology. Slices that had been incubated in DAF-2 DA and then illuminated for 5 minutes displayed increased excitability: 1) field potentials recorded in these slices displayed longer duration and often had more than one negative peak, 2) the horizontal spread of electrically-evoked activity in treated slices was larger than in control slices, and 3) the threshold concentration of the $\mathrm{GABA}_{\mathrm{A}}$ blocker picrotoxin required to induce epileptic activity was significantly reduced in treated slices.

We conclude that NO is produced by a specific subset of GABAergic neurons. These neurons are damaged during the process of DAF-2T fluorescence, and their death renders the remaining cortical tissue with higher excitability, but not sufficient to induce epileptic phenomena.

Supported by grants from the ISF and the Israeli Ministry of

Health to $Y A$

Involvement of regulatory $\mathrm{CD4}+\mathrm{CD25}+\mathrm{T}$ cells in neuronal survival after central nervous system injury and mental stress: Interaction with key neurotransmitters

Kipnis J., Mordechay S., Avidan H., and Schwartz M

Dept. of Neurobiology, Weizmann Institute of Science, Rehovot 76100.

Autoimmune neuroprotection, a term introduced by our laboratory and which would confuse scientists just four years ago, is widely used today world wide. It describes a physiological phenomenon of involvement of autoimmune $T$ cells, specific for self-proteins highly expressed at the site of CNS injury, in helping the body to withstand the consequences of destructive materials appearing from the injured neural tissue and causing secondary neuronal death. Strains of rats and mice differ in their ability to mount a physiological autoimmune response to CNS injury and therefore differ in neuronal survival after injury. Strains, able to mount an autoimmune response will benefit from higher neuronal survival, whereas autoimmune disease will not be induced in those animals. The autoimmune system is probably able to regulate itself, so that autoimmune $T$ cells will protect the damaged tissue without causing a disease. To understand the cellular regulatory mechanism, which allows an autoimmune 'benefit' without the autoimmune 'risk', we examined the role of naturally occurring regulatory $\mathrm{CD}^{+} \mathrm{CD} 25^{+} \mathrm{T}$ cells in recovery after CNS injury. We showed that depletion of these cells, which predisposes animals to development of spontaneous autoimmune diseases, enables betted and faster activation of autoimmune $T$ cells and therefore better neuronal survival. However, injection of these regulatory $T$ cells after CNS injury diminished endogenous ability to withstand secondary degenerative processes. We also showed that neurotransmitters increased after CNS injury deactivate regulatory $T$ cells, thus enabling a rapid activation of autoimmune effector $T$ cells. High sensitivity of regulatory $T$ cells to neurotransmitters might represent the 'missing link' between the immune and the nervous systems interactions. A 'fine tuning' on a level of regulatory $\mathrm{T}$ cells by neurotransmitters could be a promising therapy for neurotransmitters could be a promising

\section{Whisking behavior of freely-moving rats in an object} localization task

Knutsen P.M. Pietr M., Derdikman D. and Ahissar E.

Dept. of Neurobiology, Weizmann Institute of Sceince, Rehovot

Vibrissal touch is a major source of information about the immediate environment for rodents, many of which are nocturnal by nature. Vibrissae of rats can grow up to $\sim 6 \mathrm{~cm}$ long, and their vibrissal touch is characterized by bouts of rhythmic motions ('whisking') primarily in the range $5-11 \mathrm{~Hz}$ and up to 90 degrees of angle. The speed and amplitude of whisking movements, coupled with very rapid head and body movements, thus enables the rat to sample large regions of space in short amounts of time. In order to describe the complex whisking behavior we have devised a method, based on ultra-fast videography and image-processing techniques, which enables us to perform automated tracking of the rat's head and whisker movements. Our algorithm reduced video images of the rat into simple representations of eye, nose and whisker positions as well as whisker shape. These data enable us to precisely quantify the active, self-generated movements of whiskers and to analyze how the whiskers' trajectories and shape change upon contact with objects. This method can be applied to study detailed behavior patterns in normal animals as well as in animals with known vibrissal or motor deficits. Also, behavior can be correlated with neuronal events, such as local-field potentials and spiking events. We give examples of whisker movements in rats trained in a two-choice object-position discrimination task, and show how task performance correlates with particular patterns of self-generated movements.

\section{Blocking post-retrieval consolidation of CTA memory in}

\section{the rat induces a retrieval deficit}

Kobilo T and Dudai Y

Dept. of Neurobiology, The Weizmann Institute of Science

Experimental extinction of conditioned taste aversion (CTA) is a function of the intensity of training. We have previously shown that increasing the number of CTA training sessions from one to two is sufficient to render the retrieved trace significantly more resistant to extinction (Berman et al. Learn\&Memory 10,16, 2003). Furthermore, we have demonstrated that when the long-term trace becomes resistant to extinction, it also becomes upon retrieval sensitive anew to a consolidation blocker, anisomycin. This provides evidence for a post-retrieval or post-activation consolidation phase, i.e. 'reconsolidation' (Eisenberg et al. Science 2003, 301, 1102) We have now determined that the CTA trace that is blocked in reconsolidation does not exhibit spontaneous recovery over 11 days. This indicates that the effect of the consolidation blocker in reconsolidation is not merely to transiently suppress the expression of the long-term memory. However, the question still remains whether other treatments could unveil a latent trace. To address this question, we have used a reinstatement protocol, in which an attempt is made to reinstate a latent conditioned association by presentation of the unconditioned stimulus (in CTA, i.p. injection of $\mathrm{LiCl}$, a malaise inducing treatment), in the absence of conditioned stimulus (in CTA, taste). We found that reinstatement by such a non-associative experience can indeed partially reactivate the CTA memory 
which earlier declined after blockade of reconsolidation. Taken together with our data on reconsolidation in the medaka fish (Eisenberg and Dudai, these proceedings), our findings suggest that the blockade of post-retrieval consolidation of long-term memories somehow disrupts retrieval, and does not cause a pure storage deficit.

Supported by the Volkswagen Foundation and the Israeli Science Foundation.

CREB activation in the hippocampus, amygdala and entorhinal cortex following learning or stress procedurs Kogan I. and Richter-Levin G.

Dept. of Psychology, Brain and Behavior Research Center, Haifa University

Many learning paradigms involve also a significant component of stress. Therefore, it is important to understand the differential contribution of stress and learning to the molecular mechanisms underlying the coping response of the organism. In the present study we attempted to separate between learning and stress induced cellular mechanisms by examining the activation of the transcription factor CREB (a step in the cascade of events leading to protein synthesis) in the hippocampus, amygdala and entorhinal cortex (EC). The hippocampus is most associated with neuronal plasticity and long-term memory consolidation. There are anatomical and functional connections between the hippocampus, entorbinal cortex and the amygdala which is involved in modulating hippocampal function.

Three groups of rats were tested: 1. Learning rats - subjected to massed spatial learning protocol 2 . Stressed rats - subjected to the water maze as the learning group, but without an escape platform and without a spatial task to acquire 3 . Na\}ve rats. Learning and stressed rats were trained in the Morris water maze (in water temperature $23 \pm 1^{\circ} \mathrm{C}$ ), and 10 minutes after the last session, the dorsal CAl of the hippocampus, the amygdala and the EC were collected for analysis of CREB activation.

All the areas studied were activated following the behavioral procedures. In the dorsal CA1 CREB was activated in the learning group compared to stressed and naive animals. In the amygdala elevated levels of phosphorylated CREB were found both in learning and stressed groups. In the entorhinal cortex enhanced activation of CREB was detected in the learning group compared to naives. Additional groups of animals will be tested under more stressful conditions in order to further dissociate. learning and stress mechanisms in the aforementioned brain areas.

Supported by The Israel Science Foundation - The Charles $H$

Revson Foundation (no.582/00-1 to G.R-L.).

\section{Calcium dynamics is associated with dendritic spine} morphology

Korkotian E. and Segal M.

Dept. of Neurobiology, Weizmann Institute, Rehovot 76100

Dendritic spines have been proposed to constitute a unique calcium compartment in that they are able to raise $\left[\mathrm{Ca}^{2+}\right] \mathrm{i}$ to high levels in response to synaptic input independent of their parent dendrites. The great morphological heterogeneity among spines in the same neuron indicates that the dimensions of the spine may be extremely important for its function as a calcium compartment. Indeed, we were able to correlate the length of the spine with its possible impact on the parent dendrite, in term of $\left[\mathrm{Ca}^{2+}\right]$ diffusion between the two compartments. In different methods we found heterogeneous responses that were correlated with spine morphology. We extended these observations to examine the role of spine morphology in its functions using caged molecules. Hippocampal neurons, cultured for 2-4 weeks, were used in a confocal laser scanning microscope. A focused $\left(\leq 1 \mu^{2}\right)$ UV laser light, guided by a red laser was used to fiash photolyse caged fluorescein, for estimation of passive diffusion between spine head and dendritic shaft. Alternatively, flash photolysis of caged calcium was used to study the dynamics of decay of a momentary rise in intracellular calcium concentration. The recorded cell was filled with Alexa 546 or was transfected with red fluorescent protein, to image cell morphology, and with the calcium dye Fluo-4, to report calcium variations. The results indicate that the spine-dendrite communication varies among the different spine types. It is apparent that calcium diffusion is different from the passive diffusion of fluorescein, and that the presence of voltage gated calcium channels vary among different spines as well. These observations illustrate the importance of spine dimensions for its functions; longer spines communicate less with the parent dendrite than short ones. This communication is subject to continuous modulation by varying spine length

Supported by a grant from the Israel Academy of Science.
Retrieving Hodgkin-Huxley like models of $\mathrm{K}^{+}$and $\mathrm{Ca}^{2+}$ conductances in non-space-clamped structures Korngreen A. , Helmstaedter M. ${ }^{2}$, Sakmann B. ${ }^{2}$ and Schaefer A.T.

${ }^{1}$ Dept. of Life Science. Bar-Ilan Univ, Israel. ${ }^{2}$ Dept. of

Zellphysiologie, MPI f. Med. Res. Heidelberg, Germany

Electrophysiological properties of single neurons are crucially determined by the number and types of ionic channels expressed. Thus, for understanding single neuron function it is essential to determine exact distribution and kinetics of the relevant ionic conductances. So far the lack of space clamp has strongly limited the options to obtain correct conductance estimates and kinetic details. Here, we present an approach that retrieves accurate $\mathrm{K}^{+}$- and $\mathrm{Ca}^{2+}$ - conductance properties from voltage-clamp measurements in non-space-clamped structures. Essentially, we have implemented successive one-parameter fitting routines in NEURON to repeat previously performed experiments in a detailed compartmental model of the recorded cell. Using recorded currents as a template, this paradigm extracts correct conductance values over a wide range of experimental conditions. In particular, it is capable of retrieving complete Hodgkin-Huxley-style $\mathrm{K}^{\mathrm{f}}$-channel parameters (activation, inactivation steady-state and Additionally, $\mathrm{Ca}^{2+}$ channel parameters can be extracted with noise levels of up to 70 pA r.m.s. Complementarily to the simulation results, we have corrected voltage-clamp experiments from dendrites of cortical layer-5 pyramidal cells $(n=3)$, illustrating the feasibility of our approach. Finally, we investigated the physiological role of the increased accuracy in channel parameter measurements in full compartmentai neuronal models. Inactivation and activation parameters of $\mathrm{K}^{+}$ channels were varied and their influence on physiological measires such as size and shape of backpropagating APs was determined. The described approach will allow the fast determination of channel distributions in individual neurons and might contribute to a more complete understanding of the biophysical basis of neuronal function.

Relevance of NMDA receptor signaling pathway to genetic prediposition to anorexia nervosa: association analysis of SK3 and NR2B gene polymorphisms in family-based and

case-control population series ${ }_{\text {Koronyo-Hamaoui }{ }^{2}{ }^{2}}$, Frisch ${ }^{2}$, Barkai G. ${ }^{1,2}$, Weizman A. ${ }^{2}$ and Gak E.

${ }^{2}$ Danek Gertner Genetics Institute, Sheba Medical Center; ${ }^{2}$ Sackler Medical School, Tel Aviv University.

We investigated possible involevement of NMDA-R signaling pathway in anorexia nervosa (AN) on the basis of distribution of polymorphisms in candidate genes encoding its various components in $90 \mathrm{AN}$ family trios and case-control series. We presently report an association of two meaningful polymorphisms to $\mathrm{AN}$, a multiallelic coding $\mathrm{CAG}$ repeat in the polymorphisms to $\mathrm{AN}^{2+}$ activituated $\mathrm{K}^{+}$(SK3) channel gene and a biallelic 5073T $>\mathrm{G}$ in the 3 UTR of the NR2B subunit of NMDA-R Longer SK3 alleles, containing above the population modal of 19 CAG repeats, are significanly over-represented in AN patients, as is revealed by matched case-control analysis $(p<0.001)$ and a more robust TDT analysis of $A N$ family trios $\left(x^{2}=11.75\right.$ and $p<0.001$ upon dichotomization at 19 repeats). The distribution of SK3 alleles in the general population is distinct from $\mathrm{AN}$ and is relatively homogeneous for at least four Jewish ethnic groups. Further using TDT antalysis in AN fantily trios, reveais tiat tie $56730 \overline{\mathrm{O}}$ allele of the NR2B subunit is preferentially iransmitted to $A N$ $\left(x^{2}=5.01, p=0.025\right)$. Composite analysis inchding both, SK3 and NR2B, suggests that these factors independently contribute to the overall risk of $\mathrm{AN}$. While attributing consecutive risk scores to each of the composite SK $3 / N R 2 B$ genotypes, indicates that higher risk scores are significantly more prevalent in AN ( $p=0.001)$. Our findings might be conclusive as to the existence of consequential link between SK3 and NR2B as two factors driving towards the same psychopathological outcome. As SK channels hyperpolarize membrane potential, they may impose inhibition of the NMDA-R. Hypofunction of the NMDA-R has been implicated in the etiology of schizophrenia, however, has not been sufficiently substantiated in the context of eating disorders. Implication of NMDA-R signaling pathway in AN, might be meaningful in order to elucidate its underlying cause and appropriate treatment. 
What does it take to make a stable taste memory? Kuulmann $\mathrm{S}$. and Rosenblum $\mathrm{K}$. Center for Brain and Behavior, University of Haifa 30905

The simplest model of memory suggests that sensory information enters short term memory and later the information is transferred to long term memory, where it is permanently stored. The period of conversion short into long term memory is the consolidation phase. It is labile and highly sensitive to interference.

Pre-exposure to a novel taste in latent inhibition (LI) procedure represents a kind of incidental learning. The rats learn the new taste per se, without associating it with any external reward LI, in combination with CTA can be used to assess the familiarity of a given taste. Reduced aversion for a given taste due to LI indicates the existence of long term memory trace in the rat brain.

We aim to understand the temporal and quantitative constrains of taste memory consolidation. In the first set of experiments we determine how much input is needed to create a stable memory (i.e. $\mathrm{ml}$ of novel taste). The rats were pre exposed to $5 \mathrm{ml}(\mathrm{n}=6), 10 \mathrm{ml}(\mathrm{n}=6)$ and $15 \mathrm{ml}(\mathrm{n}=11)$ of saccharin in the $\mathrm{LI}$ paradigm. We found that $5 \mathrm{ml}$ didn't create a memory while 10 $\mathrm{ml}$ created memory and $15 \mathrm{ml}$ created stronger memory trace than $10 \mathrm{ml}$.

Second set of experiments examined whether few (3) exposures of sub threshold input $(5 \mathrm{ml})$ would differ from one exposure of the same amount of input (i.e. $15 \mathrm{ml}$ ). We observed that 3 pre-exposures during 3 successive days created a weaker memory trace than one pre-exposure of the same amount.

The third set of experiments identified the time interval of taste memory consolidation as measured in interference experiments. The rats ( $\mathrm{n}=8$ in each group) were exposed to saccharin while $5 \mathrm{hr}$ or 5 min later thev were exposed to $\mathrm{NaCl}$ U. $5 \%$ in the LI paradigm. Inere was a ciear inierierence in both groups.

We conclude that $5 \mathrm{ml}$ exposure of novel taste doesn't induce stable LTM. Repetition of sub-threshold input $(5 \mathrm{ml})$ creates weaker memory than one exposure to the same amount. In addition, an input that does induce clear LTM is still labile $5 \mathrm{hr}$ after learning.

Norepinephrine regulates genes involved in synaptic connectivity: Implications to major depression Laifenfeld D. ${ }^{1}$, Karry R. , Grauer E. 2 , Klein E. and Ben-Shachar D

${ }^{\prime}$ Laboratory of Psychohiology, Dept. of Psychiatry, Rambam Medical Center and B. Rappaport Faculty of Medicine. Technion. Haifa 31096; '2 Dept. of Pharmacology, Israel Institute for Biological Research, Ness-Ziona

A prevailing theory regards major depression as a disorder of neuronal plasticity, involving corresponding alterations in the expression of genes involved in synaptic plasticity. The present study assessed whether norepinephrine (NE), an immediate target of antidepressants, could initiate events of neuronal plasticity, and whether these could be involved in the etiology and treatment of major depression. NE treatment $\left(10^{-5} \mathrm{M} / 48 \mathrm{~h}\right)$ resulted in increased neuronal differentiation in $\mathrm{SH}-\mathrm{SY} 5 \mathrm{Y}$ neuroblastoma cells, along with an increase in the cell-adhesion molecule $L I$ (CAM-L1) in laminin and in the transcription factor cAMP response element binding protein (CREB), all involved in processes of cell survival and neurite outgrowth. We then examined whether CAM-L1, laminin, and CREB are altered in animals chronically treated with antidepressants, as well as in rats four munths after the termination of prolonged stress exposure. Antidepressants induced increases in mRNA and protein levels of CAM-LI, laminin, and pCREB, in a brain region specific manner in chronically treated rats. In contrast, a decrcase in mRNA and protein levels of CAM-LI laminin and pCREB was observed in stressed rats. Finally, the expression of CAM-L1, Jaminin and CREB was examined in 59 post-mortem specimens of psychiatric paticnts obtained from the Stanley Foundation Brain Collection, from the ventral parieto-occipital cortex, and the prefrontal cortex. Alterations were observed in CAM-LI specifically in the depressed group in both brain regions, along with less specific alterations in laminin and pCREB Moreover, medicated depressed patients differed from unmedicated patients in the expression of CAM-LI, laminin and pCREB. Taken together these results strongly implicate CAM-L1 in the pathophysiology and treatment of depression.
Nitric oxide donors attenuate dopamine - induced apototic cell deathin by maintaining the mitochondria membrane potential in a cyclic - GMP independent mechanism. Lamensdorf 1.', Agus S. ${ }^{1}$, Premkumar A. ${ }^{2}$, Gembom E. ${ }^{2}$, Simantov $\mathrm{R}^{2}$

Dept of Neurology, Hadassah University Hospital. Jerusalem. ${ }^{2}$ Dept of Molecular Genetics. Weizmann Institute of Science, Rehovot

Parkinson's disease (PD) is a neurodegenerative disease characterized by tremor, rigidity, bradykinesia, gait disturbance and postural instability. The pathological features of $P D$ are dopaminergic neuronal cell death in the substantia nigra pars compacta $(\mathrm{SNc})$ and intracytoplasmic inclusion bodies (Lewy bodies)

Nitrogen monoxide (NO) is an important biological messenger affecting multiple signal transduction pathways. It is reported to take part in MPTP and 6-hydroxydopamine (6OHDA) induced dopaminergic cell death. In view of evidence that dopamine (DA) itself can induce neuronal cell death we examined the effect of NO-donors on DA toxicity.

Human neuronal cell line (NMB) was used to examine the effect of NO-donors on DA induced apoptosis. Two NO-donors, S-nitroso-N-acetylpenicillamine (SNAP) and sodium nitroprusside (SNP) reduced DA toxicity, both however, did not attenuate mitochondrial complexes I-IV inhibitors-induced cell death.

NO-donors can attenuate apoptotic cell death in a cyclic-GMP dependent manner and/or via stabilization of mitochondrial potential. Ha et al (2003)1 demonstrated that SNAP protects the dopaminergic cell line $\mathrm{PCl} 2$ against 6OHDA in a cGMP-dependent manner 1

In the NMB cell line, 8-Br-cyclic-GMP or $1,1 \mathrm{H}-[1,2,4]$ oxadiazolo [4.3-alpha] quinoxalin-1-one (ODO) did not reduce dopamine toxicity, suggesting that the protective effect of Nu-donors was cGivip independent. Fiow cylomeiry analysis with the dye Rhodamine-123 and confocal microscopy analysis with the dye $\mathrm{JCl}$ showed that dopamine reduced mitochondrial membrane potential, and SNAP reversed this effect.

These results indicate that NO-donors have a protective effect on DA and 6OHDA induced apoptosis in this model, probably via direct effect on the mitochondrial membrane potential in a cGMP independent manner.

Reference: 1. Nitric oxide prevents 6 - hydroxydopamine induced apoptosis in PC12 cells through cGMP- dependent PI3 kinase'Akt activation. Ha et al., FASEB J. 2003. 17

$1036-1047$

Induction of plasticity by visual stimulation in the adult visual cortex revealed by intracellular recordings

Lampl I. ${ }^{1}$. Ferster D

${ }^{2}$ Dept. of Neurobiology, Institute of Science, Rehovot, 76100. Israel: Dept. of Neurobiology and Physiology Northwestern Universily. Evanston II. 60208 USA

It was assumed that any plasticity dependent on visual experience, in the primary visual cortex (V1), is limited to development stages, early in the animal life. This vicw has been changed dramatically in the past few years when various studies have suggested that the receptive field properties of neurons, even at early stages of the visual cortex can be influenced by visual stimulation. However, direct demonstration of plasticity in the primary visual cortex was limited to extracellular recordings. On the other hand many intracellular studies have characterized the mechanisms of plasticity in brain slices. To narrow the gap between the in vitro and the in vivo studies visual stimulation was combined with intracellular recordings of $V I$ neurons in ancsthetized cats. Advantaged by the recording technique. spikes can be elicited by current injection at desirable times relative to the onset of the visual stimulation. Brief presentation $(10 \mathrm{~ms})$ of optimally oriented bar, flashed on the receptive field of simple. was paired repetitively $(20 \mathrm{~Hz})$ for several seconds with a brief current pulse which induced several spikes of high frequency. A second bar, which was not paired with current injection. was used to test the effect of visual stimulation by itself. In many cells the visual synaptic response to the first bar was significantly increased while on average the response to the unpaired bar was reduced. These effects, that could last more than 15 minutes. indicate that sustained changes of synaptic strength in adult primary visual cortex can be induced by repetitive visual stimulation. 
The ascending auditory pathway: from signal detection to signal representation

Las-Ahdut L. ${ }^{1}$, Stern E.A. ${ }^{2}$, and Nelken $1^{1}$

${ }^{T}$ Dept. of physiology. Hebrew University - Hadassah Medical

School, and the Interdisciplinary Center for Neural

Computation, Hebrew University.

${ }^{2}$ Dept. of Neurology, Massachusetts General Hospital,

Harvard Medical School, Charlestown, MA, USA

Natural stimuli are often weak and accompanied by slowly-fluctuating noise. Humans and animals perceive low-level tones masked by such noise in a highly efficient manner. Neurons in primary auditory cortex (Al) tend to lock to the envelope of slowly-fluctuating noise. We have previously shown that adding low-level tones qualitatively changes these responses by suppressing envelope locking, causing the responses to be more similar to the responses to a tone in silence. Here we study the evolution of this suppression in the ascending auditory system using intracellular recordings in vivo from $\mathrm{A} 1$ and extracellular recordings from two preceding subcortical auditory nuclei, the Medial Geniculate Body (MGB) and the Inferior Colliculus (IC). Hypersensitive suppression was present in the subthrehold responses of all Al neurons that showed envelope locking, and in some MGB neurons; suppression often occurred below tone threshold in silence. In contrast, the locking of IC neurons was only weakly affected by low-level tones. These results show that the neuronal representation of weak tones in strong slowly-fluctuating noise is highly amplified along the ascending IC-MGB-AI auditory axis. We interpret this hypersensitive suppression as a correlate of the representation of the tone as a distinct auditory object, which is gradually built along the ascending auditory pathway.

Development of an in vitro blood-brain barrier model using PC12EN endothelial cells

Lecht $S$. Tabakman R. ${ }^{1}$, Harriton-Gazall $E^{2}$, Gilon $\mathrm{C}^{2}$ Sterin-Cohen $M^{1}{ }^{1}$, Ringel I. ${ }^{1}$, Reich R. ${ }^{1}$ and Lazarovici $P^{1}{ }^{1}$ Depts. of Pharmacology', School of Pharmacy. Dept. of Organic Chemistry'. Institute of Chemistry, The Alexander Silberman Institute of Life Sciences The Hebrew University of Jerusalem

Blood-brain barrier (BBB) separates the brain parenchyma from cerebral microvessel blood environment. It ensures that only vital nutrients and factors will be transported into the brain, by utilizing a wide variety of transporters i.e. glucose, nucleoside large neutral amino acid transporters, etc. The transport from the brain to blood of toxic metabolites or xenobiotics is mediated by efficient efflux transporters i.e. PgP, MRP, etc. Another feature of BBB is represented by its very low paracellular permeability in contrast to peripheral capillaries. These important anatomical and physiological characteristics are due to specialized structures of the cerebral microvessels and unique regulatory factors. The in vitro BBB models available to date are disputable due to the lack of pharmacological characterization.

In the present study a unique PC12EN cell line, generated by hybridization (fusion) between rat pheochromocytoma cell line (PC12) and bovine adrenal medullar endothelial cells (BAME), was used $(1,2)$. The main goal was to develop an in vitro $\mathrm{BBB}$ model using a commercial double chamber technology (e.o. Transwell ${ }^{2}$ ) and PC12EN endothelial cells To accomplish this goal, the optimal culture conditions characterized by measurements of trans endothclial electrical resistance (TEER), that enable barrier properties, were defined. The paracellular transport through $\mathrm{PC} 12 \mathrm{EN}$ monolayer was evaluated with FITC-markers of different molecular weigh such as bovine serum albumin (BSA $66 \mathrm{kDa}$ ) and fluorescein $(0.3 \mathrm{kDa})$. The paracellular permeability was low. indicative of

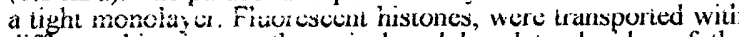
different kinetics on the apical and basolateral sides of the cndothelial cells with an increased transport from the basolateral to the apical side of the monolayer. These experiments indicate the functional asymmetry of PC12EN monolayer. Phospholipids-based pro-drug DP-524 with a shorter falty acid on $\mathrm{C} 2$ position was higher transported transcellularly than DP-487, with a longer fatty acid on C2 position (compounds kindly provided by D-Pharm Ltd.). These experiments may suggest that DP phospholipids arc transported differentially by the PC12EN monolayer utilizing a specific carricr which deserves further investigation. PC 12EN represent a novel endothelial cell line for future developmen of an in vitro $\mathrm{BBB}$ model to be used in CNS drug discovery programs.

1 Rasouly D. et. al (1996) Staurosporine induces neurite outgrowth in neuronal hybrids (PC 2EN) lacking NGF receptors. $J$. Cell Biochem. 62,356-371;2. Jiang H. cl. al (1997) Expression of human p140trk receptors in pl 40 trk-deficient, $\mathrm{PCI}$ 2/endothelial cells results in nerve growth factor-induced signal transduction and DNA synthesis. J. Cell Biochem. 66,229-244.

Supported by PharmaLogica consortium in the frame of MAGNET program of the Israeli Ministry of Industry and Commerce.

Area-specific deprivation effects in occipito-temporal cortex of human amblyopes

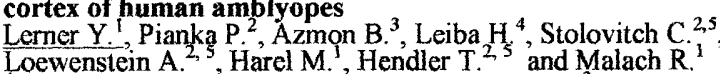
${ }^{2}$ Weizmann Institute of Science, Rehovot, 76100; ${ }^{2}$ Sourasky Medical Center, Tel Aviv, 64239; ${ }^{3}$ Notal Vision $\mathrm{Co}_{5}$, Tel Aviv, 63143; ${ }^{4}$ Kaplan Medical Center, Rehovot, 76100; ${ }^{5}$ Tel-Aviv University, Tel Aviv

Amblyopia is a condition characterized by low visual acuity of cortical origin suggestive of a deficit of central visual field processing, which provides a striking example of the influence of abnormal early visual experience on human vision. Recently, in normal subjects, we have shown that certain object images, e.g. faces and buildings, could be differentially associated with central and peripheral visual field biased representations. Such association predicts that amblyopia could selectively affect representations of faces compared to building images. Here we studied this possibility using fMRI. Amblyopic subjects $(n=17)$ were tested while receiving monocular stimulation of face and building images through either eye. In control experiments, a blurred version of the same images, in which we simulated the level of contrast sensitivity deficit of the amblyopic condition, was used. $<\mathrm{O}: \mathrm{P}</ \mathrm{O}: \mathrm{P}$

Our results revealed that amblyopia produces a selective deficit in face-related (posterior fusiform gyrus (pFs) and lateral occipital area (LO)) compared to building-related (collateral (COS) and transverse-occipital (TOS) sulci) regions. The amblyopic effect depended on both object category and cortical region - the face-related amblyopic effect was found only in face-related regions, and not in building-related regions. These findings are compatible with the concept that face processing depends on high-acuity central vision whereas building-related processes are more peripherally biased. The results also point to the importance of early visual experience in establishing proper functioning of high order face
representations. Behavioral performance was nicely compatible with the fMRI results.

Supported by ISF grant 77/00, Adams Super Center for brain studies. Tel Aviv University grant and Center of Excellence grant 8009

Hippocampal damage in offspring that experience maternal inflammation during pregnancy

Lev V. ${ }^{1}$, Huleihel $\mathrm{M}^{2}$, Hallak M. ${ }^{3}$, Sorokin $\mathrm{Y}^{5}$ and Golan $\mathrm{H}^{1}{ }^{1}$

Zlotowski ctr. for Neuroscience ${ }^{l}$. Dept. of Immunol. ${ }^{2}$ and Dept. of Obs. \& Gyn ${ }^{3}$. Fac. of Health Sciences and, BGU.

Beer-Sheva and Dept. of Obs. \& (ryn. Hutzel Hospital.

Detroit

Intrauterine inflammation is associated with neurodevelopmental damage and increased risk for reduced intelectual achievements in newborns. We have previosly shown that systemic maternal inflammation, induced by intraperitoneal (i.p) injection of lipopolysaccharide (LPS, 0.12 microgr/gr) to 17 gestation day mice (E17). increased proinflammatory cytokins levels in embryo brains. In the present study we used the same inflammation system and evaluats the histopathological damage in brains of offspring at postnatal day 7 (P7), P14, PI80. PFA fixed, paraffin embedded, sagital sections, $4 \mu$ thick, were stained with Niss staining and analyzed. The most sensitive brain area inspected was the hippocampus. Significant differences in CAl cell laver thickness were observed at P7 and P14, while at P180 - no differences were observed in CAI region thickness between offspring to LPS treated mothers and control. However cearfull examination of $\mathrm{CAl}$ region revealed shrinking of pyramidal cells ( $33.5 \pm 4.3 \mu^{2}$ control and $16.4 \pm 1.5 \mu^{2}$ LPS $\beta_{<0.001)}$ and increased cell density $\left(0.033 \pm 0.004 \mathrm{cell} / \mu^{2}\right.$ and $0.064 \pm 0.005 \mathrm{cel} 1 / \mu^{2}$, respectively, $P<0.01$ ). In addition, in the dentate gyrus region, the granular cell layer was significantly thicker and longer in LPS offsprings as compared to control, al $\mathrm{P} 14$ and P180 $(\mathrm{P}<0,001)$ In this region. at P180, no differences were observed in granular cells size or density indicating that the difference in layer thickness and lengih resulted from increase in granular cell number. The present study indicate a distruction of neurogenesis in the hippocampus of offsprings subject to prenatal inflammatory response. These results support our previous observations of altered learning and memory in these mice

Supported by BSF grant contract No. 200172 
Exposure to Vigabatrin modulates reflex development and brain morphology in newborn mice

Levay T. Saar T.. Berkowitz L and Golan H.

Dept. of Developmental Molecular Genetic. Faculty of Health Sciences and Zlotowski center. for Neuroscience, Ben Gurion Unversity Beer-Sheva

Epilepsy is the most common neurological disorder in young numans. Anti-epileptic drugs (AEDS), used to trear seizures in chinưren, infants, and pregnami women, cause cognitive impairments, microcephaly and birth defects. The cause of unwanted effects of therapy with AEDs is unknown. Vigabatrin (VGB) belongs to a new class of anti-epileptic drugs used widely in children with infantile spasms and is considered as a safe therapy. VGB blocks the enzyme GABA-transaminase, thereby unhibiting GABA degradation.

In the present study we examined the influence of exposure to VGB in two time periods during early postnatal age, on morphogenic parameters, reflex development and brain morphology in newborn balb/c mice. Newborn mice were injected (subcutaneous) daily with VGB (50mg/kg) - during
postnatal days 1 (P1)-P7 (VGB) and P4-P14 (VGB'). Control (CT) newborns were injected with a similar quantity of vehicle solution, during similar time periods. Body weight gain of newborns treated with VGB was lower compared to $\mathrm{CT}$ groups (0.51gr, VGB; $0.61 \mathrm{gr}$, VGB'; $0.66 \mathrm{gr}$ and $0.73 \mathrm{CTs}$ ). Teething began two days earlier in both treated groups, compared to CTs, however hair growth was influenced differentially by the two treatments. Examination of reflex development showed that in the righting reflex the percent of newborns succeeding in the task was the lowest in the VGB group, while the newborns in the VGB' group did not differ from the CTs. A similar tendency was seen in the time required for righting. On the rotarod task, an additional motor reflex developing during CT (held more than 180 degrees moto a rofiting rod heginning at $\mathrm{P} 12$ and the CT at P10). The cliff avoidance reflex was at $\mathrm{P} 12$ and the $C T$ at $P 10$. The cliff avoidance reflex was morphology on P7 showed similar cortex lengths and widths and cerebellar area in VGB' and CT. In contrast, CAl cell layer in the hippocampus was thicker in the VGB' group compared to $\mathrm{CP}^{-}$(1022 and 782 microns, respectively). The Dentate Gyrus granular cell layer was thinner in the $\mathrm{VGB}^{\prime}$ group compared to CT ( 957.6 and 1120 microns, respectively). In conclusion, early postnatal exposure to Vigabatrin modifies reflex development and hippocampal morphology, and might have long term consequences on mice behavior.

The role of environmental stimulation on neuronal plasticity in young ApoE transgenic mice. Levi O. ${ }^{3}$, Jongen-Relo A.L. ${ }^{2}$, Feldon J. ${ }^{2}$, Roses A.D. ${ }^{3}$ and Michaelson DM ${ }^{1}$

'Dept. of Neurobiochemistry, George S. Wise Faculty of Life Sciences. Tel Aviv University, Ramat Aviv 69978, Israel: ${ }^{2}$ Dept. of Biology, Laboratory of Behavioral Neurobiology, The Swiss Federal Institute of Technology, Zu rich, Switzerland; 3Dept. of Neurology, Duke University, Durham, $N C$, USA

Evidence for structural modification in the brain following environmental stimulation has been provided during the last decades. These plastic changes are supposed to reflect reorganization of neuronal connections involved in postnatal development and adult adjustment involved in the process of learning and memory. Furthermore, clinical evidence showing an association between higher educational attainment and reduced risk for Alzheimer's disease (AD) indicates that a stimulating environment has a positive effect on cerebral health. The apolipoprotein E4 (apoE4) genotype is a major risk frotor for Alzhoiner's disease; However, the mochanism is unknown. We have recently presented results showing that exposure of young mice ( 1 month old) transgenic to the human apoE isoforms to an enriched environment (EE) for a period of 5 months resulted in an improvement of learning and memory abilities in apoE3 transgenic mice, (as measured by wet $T$ maze), whereas mice transgenic for the human apoE4 were unaffected by this environmental stimulation. These cognitive effects were associated with higher hippocampal levels of the presynaptic protein synaptophysin and of NGF in apoE3 but not in apoE4 transgenic mice.

In the present work we demonstrate that these human apoE isoforms differentially modulate neurite outgrowth in the hippocampus of young apoE transgenic mice after exposure to a complex environment. The apoE3 but not the apoE4 transgenic mice showed increased levels of the dendritic marker MAP-2 and the axonal marker TAU-1. These changes were accompanied by changes in the levels of the low-density lipoprotein receptor-related protein (LRP). These findings suggest that apoE4 inhibits these beneficial effects of the enriched environment on Hippocampal neurite outgrowth and that LRP may have a role in these phenomena.
Activation patterns in visual cortex following optic neuritis: An fMRI study 2

${ }^{1}$ Dept. of Neurology. Hadassah - Hebrew University Hospital. Jerusalem: 'Neurobiology Dept., Hebrew University,

jerusalem

Objectives: To evaluate the distribution of cortical activation in the visual pathways in patients atter an acute optic neurtus episoúe.

Background: Optic neuritis is a common condrtion that causes a reversible loss of vision. Typically, vision deteriorates over a few days to 2 weeks before spontaneous improvement takes place. Improvement in vision is seen up to a year after the acute episode. Recovery to normal or near normal visual acuity is usual, despite the frequent persistence of conduction abnormalities as evidenced by prolonged visual evoked potential (VEP) latencies. It is known that remyelination of the nerves takes place, leading to improvement of visual function (i.e peripheral effects). However, central reorganization processes at higher cortical level may also contribute to the recovery process. Our aim was to assess this issue, using fMRI, by comparing the pattern of activation in the visua cortex in the affected and normal eye

Methods: ARI was utilized to obtain brain activation induced by epochs of object stimuli, scrambled objects, and checkerboard patterns. Activation in primary and higher visual areas was assessed in 3 subjects suffering from unilateral optic -eusitis and prolonged VEP latencies.

Resulws: In all patients, reduced activation was seen upon stimulation of the affected eye in the early retinotopic visual arcas. In contrast, highcr objcct related visual areas showed no difference in the activation level between the two eyes

Conclusions: An acute episode of optic neuritis (which lead to reauced activation in primary visuai areas) ád not aiter the response oi higher objeci reiaied areas. This may inäicate a buit-in robustness of these areas to disruption of the visual input. Alternatively, it could reflect an adaptive functional reorganization of the cortical response to an abnormal input.

Supported by the Israel Science Foundation (ISF) Grant $\# 8009$

Involvement of poly ADP-ribosylation in memory formation in Aplysia

Levitan D. Susswein A.J., Katzoff A., Schwartz JH,

Visochek L., Cohen-Armon M.

Fac Life Sci, Bar Ilan Univ, Ramat Gan 52900, Il; Neufeld Card Res Inst, Tel Aviv Univ, Tel Aviv 69978, 11 ; Cntr Neurobiol Behav, Columbia Univ. New York, NY 10032 USA

PolyADP-ribosylation (pAR) is a transient posttranslational modification of DNA-bound proteins that modulates chromatin structure. It is catalyzed primarily by
polyADP-ribose-polymerase-1 (PARP-1). We examined pAR of PARP1 and of linker histone $\mathrm{H} 1$ in response to training causing long term sensitization of the tail and siphon withdrawal reflexes, and to its neural correlate, long-term facilitation of sensory to motor neuron synapses. Training consisted of 4 spaced shocks to one side of the body wall of Aplysia, which elicits ipsilateral long-term sensitization of withdrawal reflexes, and ipsilateral long-term facilitation of sensory to motor neuron synapses. This procedure caused an increased PAR of PARP1 in the pleural and pedal ganglia ipsilateral, but not contralateral to the shock. Long-term facilitation elicited by 5 spaced pulses of 5 -HT to the isolated pleural-pedal ganglia also caused increased pAR of PARPI and of linker histone $\mathrm{HI}$. In contrast, one pulse of 5-HT, which causes short-term facilitation, did not cause increased pAR. The increases in $\bar{p} A \bar{K}$ were suppressed by pretreatment with 3-aminobenzamide (3-AB), an inhibitor of $p A R$. In addition, 5 spaced applications of FMRF-amide, which causes long-term depression of sensory to motor neuron synapses, suppressed DAR of PARP

We also examined pAR after another form of memory. Aplysia learn to decrease their responses to inedible food. Training leads to short- and long-term memory. Immediately after training there was an increase in pAR in the cerebral and buccal ganglia controlling feeding. There was no increased pAR after animals were stimulated with food (and responded with feeding movements) for a time equivalent to that needed for training. Treatment with 3-AB blocked $\mathrm{pAR}$ after training, and also blocked long-term memory without affecting either the animal s behavior during training or short-term memory. Thus, $p A R$ is a required step in the formation of long-term, but not short-term memory

pAR has previously been associated with cellular responses to DNA damage. Our results suggest that modification of nuclear proteins by pAR also has a role in long-term memon, presumably by altering chromatin structure, and thereby regulating gene expression. 
Differential induction of apoptosis in rat glioma cells: Evidence for p-c-Jun, Cytochrome -c and Caspase 3 involvement

Levkovitz $\mathrm{Y}^{1,2}$, Gil-Ad I. ${ }^{1}$, Zeldich E. ${ }^{1}$, Dayag M. ${ }^{1}$ and Weizman A.

Laboratory of Biological Psychiatry. Felsenstein Medical Research Center, Campus Rabin, Petah-Tiqua 49100; ${ }^{2}$ Shalvata Mental Health Center, and Sackler School of Medicine, Tel-Aviv University, Tel Aviv

Antidepressants are drugs widely used for the treatment of mood disorders. Antidepressants vary on their pharmacology and neurochemical effects with tricyclic antidepressants (TCAs) being less specific in transporter and receptor blockade than newer drugs such as the selective serotonin reuptake inhibitors (SSRI).

Recent studies have shown that some TCAs and SSRI's have potent apoptotic activity but, the detailed mechanism and the major early key players underlying this activity remain unclear.

In the present study we found that various classes of antidepressants differentially affect the survival of rat $\mathrm{C} 6$ glioma and human neuroblastoma cells. The SSRI's (paroxetine and fluoxetine) and the TCA (chlomipramine) but not the TCA (imipramine) and the atypical antideprresant (mianserin) caused a concentration dependent cell death. The potency of the effect was in the following order: paroxetine $>$ chlomiparamine $>$ fluoxetine. Our results strongly imply involvement of apoptotic mechanism, by the findings that: 1 . The antidepressant-induced cell death was accompanied by DNA fragmentation as reflected by the flow cytometry of PI stained C6 cells. 2. The combined staining of Hoechst and PI show a typical PI staining of fragmented nuclei in cells treated with the selected antidepressants. These apoptotic changes anticipatc by rapid increased in p-c-Jun levels, cytocrome $c$ release from mitochondria and caspase 3 activation.

In conclusion, we have demonstrated that selected antidepressant agents belonging to the SSRI's class are potent inducers of apoptosis in glial and neural cell lines.

We provide evidence which strongly implicates activation of p-c-fun and subsequent increased Cyt $\mathrm{C}$ release followed by caspase 3 activation in this apoptotic pathway. More studies are needed to explore whether this process has a clinical implication for potential anti-tumor activity in the brain.

Differentiation of stem cells from human bone marrow to dopaminergic-like neurons

Levy Y.S. ${ }^{1}$, Burshtein A. , Bulvik S. ${ }^{2}$, Ashery U. ${ }^{3}$, Barhum Y. Melamed E. and Orfen D.

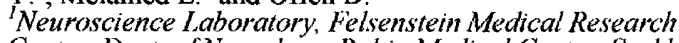
Center, Dept. of Neurology, Rabin Medical (enter, Sackler School of Medicine, Tel Aviv University. Tel Aviv'Laniado Medical Center, Natanya; ${ }^{3}$ Dept. of Neurobiochemistry Wise

Faculty of Life Sciences, Tel Aviv University, Tel Aviv

Human mesenchymal stem cells present in adult marrow are multipotent stem cells that can differentiate into bone cartilage, fat, tendon and muscle. Recently, human bone marrow stromal cells (hBMSc) have been shown to be able to differentiate into hepatocytes, myocytes and neural cells. In our study, we investigated the possible use of hBMSc in cell therapy for Parkinson's disease. We incubated cells from human bone marrow, aspirates from iliac crest, with retinoic acid, butylate hydroxyanisole and increased intracellular cyclic AMP. We found that hBMSc changed their phenotype to neural cells after 24 hours and expressed various neuronal markers. The differentiated cells demonstrated a resting membrane potential of $-70 \mathrm{mV}$, rectified current in response to increased voltage, and intra-cellular calcium increase following potassium challenge. Moreover, the differentiated hBMSc expressed tyrosine hydroxylase, and dopa decarboxylase, the key enzymes of dopamine synthesis. The cells also demonstrated other markers for dopaminergic neurons such as D2 dopamine receptor, dopamine transporter, and vesicular monoamine transporter-2. Furthermore a significant amount of dopamine precursors and metabolites were secreted to the medium, as measured by HPLC analysis. Our results indicate that dopaminergic-like cells might be derived from human bone marrow and could possibly be used for autologous transplantation in Parkinsonian patients.

Strain-dependent differences in $T$ cell-inflammation after CNS injury: Involvement of microglia

Lewitus G.M., Kipnis J., Avidan H., Mordechay S. and Schwartz M.

Dept. of Neurobiology, Weizmann Institute of Scince, Rehovor It was previously shown by our group that CNS injury can benefit from autoimmune T-cell. Immune deficient animals showed worse neuronal survival after CNS injury. A correlation was found between ability to withstand neuronal insult and local inflammatory response in terms of onset. intensity and duration of the response. Strains of mice and rats were shown to differ in their ability to manifest a spontaneous $\mathrm{T}$ cell-dependent ncuroprotective response after injury. An understanding of the basis for such strain differences is still missing in terms of antigenic specificity, phenotype or state of activation. Here we show that the phenotype of T cells homing to injured spinal cord differs among strains. $T$ cells were isolated from injured spinal cords of two mouse strains differing in their ability to mount a protective $T$ cell response, using affinity columns. Among the isolated $\mathrm{CD} 4+$ cells, more than $57 \%$ are memory/activated $\mathrm{CD} 4+(\mathrm{CD} 44 \mathrm{hi} \mathrm{CD} 45 \%$ ) cells in both strains, in contrast to the lymph nodes, where the ratio is $20 \%$ of memory/activated CD $4+\mathrm{T}$ cells and $80 \%$ nałve $\mathrm{CD} 4+\mathrm{T}$ cells (CD44lo CD45hi). However, mice with better ability to mount neuroprotective response following injury showed increased abundance of activated cells (CD69+) among the memory/activated phenotype. The presence of a higher incidence of activated $T$ cells at the early stage following the injury can explain a better control of local microglia response and thus better recovery.

\section{TRP channels may play a role in the response of layer 5} neocortical neurons to hypoxia

Libman L., Gutnick M.J., Levavi-Sivan B. and Fleidervish I.A Koret School of Veterinary Medicine \& Dept. of Animal

Sciences, the Hebrew University of Jemisalem. Rehovot

The rapid and devastating consequences of hypoxia for forebrain function in mammals reflects the sensitivity of synaptic and intrinsic membrane properties of cortical neurons to metabolic insult Because the properties of Transient Receptor Potential (TRP) channels in Drosophila retina have been shown to be very sensitive to metabolic stress, we have investigated whether these calcium and monovalent cation-permeable channels may also be involved in the response of neocortical neurons to hypoxia. TRP channels of various types are widely distributed in mammalian tissue, including brain. Using Western blots with TRPC antibodies. we found high levels of TRPC $1,3,5$ and 6 in neocortical microsomes, while TRPC4 was notably absent. In whole cell recordings from layer 5 pvramidal neurons in neocortical brain slices, blockade of voltage dependent $\mathrm{Na}^{+}, \mathrm{K}^{+}$and $\mathrm{Ca}^{2+}$ channels revealed a large. non-specific cationic current $\left(\mathrm{I}_{\text {cat }}\right)$. several of whose properties were similar to those previously reported for TRP-mediated currents. Thus. the current was inhibited by $\mathrm{La}^{3+}$, and displayed a characteristic, outwardly rectifying current-voltage relationship. The characteristics of $I_{\text {cat }}$ were rapidly altered during hypoxic episodes, which we induced by switching the gas flow to $95 \% \mathrm{~N}_{2}-5 \% \mathrm{CO}_{2}$. Within 120 seconds of hypoxia onset, there was a significant decrease in outward $I_{\text {cat }}$ at depolarizing voltages, and a parallel marked change in the shape of the current-voltage relation, as the inward current at negative voltages increased. The effects were rapidly and completely reversed upon reoxygenation when hypoxic episodes lasted less than 5 minutes. These changes in the properties of a putative TRP channel-mediated current indicate a significant increase in persistent inward cationic conductance at subthreshold voltages, which would be cxpected to contribute to neuronal vulnerability during oxygen deprivation by providing a route for $\mathrm{Na}^{+}$and $\mathrm{Ca}^{2+}$ entrance.

\section{Characterization of the effect of paired associative} stimulation on somatosensory evoked potentials in human subjects

Litvak V., Schramm A., Stefan K., Pratt H.. Zaaroor M. and Classen $\mathrm{J}$.

Dept. of Biomedical Engineering, Dept. of Medicine, Evoked Potentials Laboratory - Technion-Israel Institute of

Technology. Haifa, Isracl: Dept. of Neurology, Human Cortical Physiology' and Motor Control

Laboratory. University of Wuerzburg. Wuerzhurg. Germany

Pairing protocols combining electrical stimulation of a peripheral nerve with transcranial magnetic stimulation (TMS) of the cortex have recently been shown to modity the amplitude of motor evoked potentials (MEPs) and somatosensory evoked potentials (SEPs) in healthy human subjects. There is experimental evidence that this phenomenon is related to spike timing dependent synaptic plasticity at the cellular level. Our goal in thepresent study was to characterize the effect of paired associative stimulation (PAS) on SEPs by means of multichannel electroencephalographv(EEG). EEG was recorded before and after paired stimulation of the right median nerve and left somatosensory cortex in healthy volunteers. Shortlasting modification of SEP amplitude was successfully induced in themajority of the subjects. The associated changes in scalp distribution andspectral characteristics of the EEG activity were quantified and related to the SEP changes. 
Impairment of episodic and spatial memory in rats by selective inhibition of cerebral cytochrome oxidase Luques L. ${ }^{1}$, Magen I ${ }^{1}$, Shoham S. and Weinstock M. Dept. of Pharmacology, Faculty of Medicine. Hebrew University; ${ }^{2}$ Research Dept., Herzog Hospital, Jerusalem

Oxidative stress occurs at an early stage in Alzheimer's disease (AD) and may be associated with abnormalities in mitochondrial function. Cytochrome oxidase (COx) plays an important role in aerobic energy metabolism and its activity is reduced in $A D$. $C O x$ can be selectively inhibited in brain mitochondria by sodium azide (NaAz) that binds to the electron carrier of the heme prosthetic group. The present study examined the effect of chronic $\mathrm{NaAz}$ administration on $\mathrm{COx}$ in discrete brain areas and on spatial and episodic memory that are primarily affected in AD. Male rats (15) weighing $330-350 \mathrm{~g}$ were implanted sc with Alzet minipumps containing $\mathrm{NaAz}$ released at the rate of $1 \mathrm{mg} / \mathrm{kg} / \mathrm{hr}$. Control rats (11) were implanted with pumps containing saline. Episodic memory was assessed 3 and 5 weeks later by the object recognition test, and spatial memory 6 weeks later by the Morris water maze (MWM) test. Rats were then deeply anesthetised perfused transcardially with paraformaldehyde and brains sectioned. $\mathrm{COx}$ showed differential staining in control rats, with higher intensity in layers 3 and 5 frontal cortex and in layers 3-4 in temporal cortex. Densitometric image analysis revealed that $\mathrm{NaAz}$ lowered $\mathrm{COx}$ activity significantly in layer 3 in both regions. Cresyl violet staining did not reveal a loss of cells in any brain region. COx inhibition significantly impaired episodic memory 3 and 5 weeks, and both working and reference memory 6 weeks after instillation of the NaAz pump. Our findings show that NaAz-induced reduction in $\mathrm{COx}$ activity can lead to significant impairment in both spatial and episodic memory in the absence of detectable neuronal loss. This may occur through reduction in the ability of dendrites to process synaptic inputs from subcortical and/or cortico-cortical pathways. $\mathrm{COx}$ inhibition by $\mathrm{NaAz}$ could provide a relevant animal model for testing potential agents with neuroprotective effects against the consequences of mitochondrial dysfunction.

Rivastigmine corrects the episodic and spatial memory deficit induced in rats by chronic cerebral hypoperfusion Magen $1^{1}$. Shoham $S^{2}$, Poltyrev $T^{1}$ and Weinstock $M$. Tept of Pharmacology, Faculty of Medicine, Hebrew University; ${ }^{2}$ Research Dept., Herzog Hospital, Jerusalem Cerebral hypoperfusion occurs during aging, Alzheimer (AD) and vascular dementia (VD) and may contribute to neuronal damage. Permanent bilateral carotid artery occlusion $(2 \mathrm{VO})$ in rats has been proposed as a model of pathological changes in dementia. It reduces cortical blood flow and glucose utilisation, causes a progressive impairment in spatial memory and reduction in hippocampal and cortical acetylcholine, apparently in the absence of overt neuronal damage. We investigated the effect of rivastigmine, a cholinesterase inhibitor currently used for the treatment of $A D$ and $V D$ on both spatial and episodic memory impairment induced by $2 \mathrm{VO}$ in rats. A careful histological analysis was performed to see if any neurodegeneration could be detected. Male rats (300-350g) were subjected to $2 \mathrm{VO}$ or sham operation under Equithesin anesthesia. Episodic memory that depends on intact cortical activity was tested 3 weeks after surgery by the object recognition test, and spatial memory, dependent on hippocampal function, was tested at 4 and 6 weeks by the holeboard and Morris water maze tests, respectively. Rivastigmine $(1 \mathrm{mg} / \mathrm{kg}$ ) was injected sc. $45 \mathrm{~min}$ before each test. Rats were anesthetised 4 months later and brains fixed by transcardial perfusion with paraformaldehyde. Astrocytes were stained by mouse anti-glial fibrillary acidic protein and microglia by mouse anti-complement 3-receptor (clone OX-42). Image analysis revealed for the first time, activation of recident microglia (but not astrocytes) in 2VO rats, in hippocampal CA1-2 field, but not in parietal cortex. This may reflect synaptic loss and impaired hippocampal neuronal activity underlying the significant impairment induced by $2 \mathrm{VO}$ of both episodic and spatial working memory. Memory deficits were corrected in all tests by rivastigmine. The findings support the suggestion that chronic cerebral hypoperfusion induces neuronal deficits in cortical and hippocampal cholinergic transmission that contribute to the memory deficits.

Spreading depression in-vivo is associated with disturbance at the blood-brain-barrier

Major S. ${ }^{2}$. Friedman A ${ }^{2,3}, 3$ and Dreier J.P. ${ }^{1}$

Major S., Friedman A. Physiology, Charit', Humboldt University, Berlin, Germany; ${ }^{3}$ Zlotowski Center of Neuroscience, Ben Gurion University, Beer Sheva, Israel

Cortical spreading depression (CSD) is a depolarization wave propagating at a rate of $-3 \mathrm{~mm} / \mathrm{min}$ in the cerebral cortex. The phenomenon is triggered by electrical, mechanical or toxic factors and is probably involved in the pathogenesis of focal ischemia, head trauma and migraine. Endothelin-1 (ET-1), a vasoactive peptide and astroglial/neuronal modulator has been recently shown to disrupt the blood-brain-barrier (BBB) as well as to potently induce CSD in rat in-vivo. Here we investigated whether the co-appearance of CSD and BBB opening is specific for ET- 1 or a more general characteristic of CSD. Thus, CSD was triggered by high- $\mathrm{K}^{+}$solution in the open cranial window of anesthesized (thiopental, $100 \mathrm{mg} / \mathrm{kg}$ BW i.p.), tracheotomized and artificially ventilated rats. CSDs were monitored using a $\mathrm{K}^{+}$sensitive microelectrode and a subarachnoid $\mathrm{Ag}-\mathrm{Cl}$ electrode. Cerebral blood flow was recorded using Laser Doppler flowmetry (LDF). BBB disruption was quantitatively estimated using image analyses in brain sections following the peripheral injection of the non-permeable fluoresceine isothiocyanate and/or the albumin-binding Evan's Blue. Increased BBB permeability was observed in the cortical region locally superfused with $\mathrm{K}$ to induce CSD. Moreover, we not only found dye extravasation in the area where $\mathrm{K}^{+}$was topically applied but also to a somewhat lesser degree in the other regions of the same hemisphere undergoing CSD. Our data suggest that CSD by itself, propagating through naive cortical tissue, disrupts BBB.

Phosphatidylinositol 3-kinase (PI3K), phosphoinositide-specific phospholipase- $\mathrm{Cg}$ (PLCg) and onstein kinase-C (PKC) signal myelin phagocytosis meais ted by complement receptor-3 alone and combined with scavenger receptor-AI/II in macrophages

Makranz $C_{1}^{1}$, Cohen G. ${ }^{1}$, Baron A. , Levidor L. ${ }^{1}$, Kodama T. ${ }^{2}$,

Reichert $\mathrm{F}^{1}$ and Rotshenker $\mathrm{S}$

${ }^{1}$ Dept. of Anatomy \& Cell Biology, Hebrew University Hadassah Medical School, Jerusalem, Israel; ${ }^{2}$ Lab. for Systems Biology and Medicine, U. of Tokyo, Tokyo, Japan

Complement-receptor-3 (CR3/MAC-1), scavenger - receptor $\mathrm{Al} / \mathrm{II}$ (SRAI/II) and Fcy-receptor ( $\mathrm{F} c \gamma \mathrm{R}$ ) can mediate phagocytosis of degenerated myelin in macrophages and microglia. However, CR3/MAC-1 and SRAI/II, but not FcyR, mediate phagocytosis after axonal injury. We tested for PI3K, PLC $\gamma$ and PKC signaling in myelin phagocytosis mediated by CR3/MAC-1 alone and by CR3/MAC-1 combined with SRAV/II. Phagocytosis was inhibited by PI3K inhibitors wortmannin and LY-294002, PLC $\gamma$ inhibitor U-73122, classical PKC (cPKC) inhibitor Go-6976, general PKC inhibitors Ro-318220 and calphostin-C, and BAPTA/AM which chelates intracellular $\mathrm{Ca}^{+2}$ required for $\mathrm{cPKC}$ activation. PKC activator PMA augmented phagocytosis and further alleviated inhibitions induced by PI3K and PLC $\gamma$ inhibitors. Overall altering PKC activity modulated phagocytosis four- to six-fold between inhibition and augmentation. PLC $\gamma$ activation did not require tyrosine phosphorylation. Thus signaling of myelin phagocytosis mediated by CR3/MAC-1 alone and by CR3/MAC-1 combined with SRAL/II involves PI3K, PLC $\gamma$ and cPKC, the cascade PI3K>"PLC $y>$ cPKC, and wide-range modulation by PKC. This pathway may thus be targeted for in vivo modulation, which may explain differences in the efficiency of CR3/MAC-1 mediated myelin phagocytosis in different pathological conditions.

\section{Do We have two different genetic animal models of} childhood depression?

Malkesman O., Braw Y., Maayan R., Yadid G., Shabat-Simon M. Kesner Y., Touati D., Overstreet D. H. and Weller A.

Bar-Ilan Uiversity, Ramat Gan,., Israel; Tel-Aviv University,

Israel; UNC, USA

Childhood depression has received attention as a distinct clinical entity only relatively recently. We examined whether existing genetic animal models of depression used in psychopharmacological research in adult rats are also valid for modeling depression in juvenile rats. Two different "depressed" lines were studied: the Flinders Sensitive Line (FSL) and their controls. Sprague-Dawley (SD) rats, and the Wistar Kyoto (WKY) line and their controls, Wistar rats. Adults from FSL and WKY lines have been shown to demonstrate depression-like symptoms, e.g., increased immobility in the swim test, prevented by chronic antidepressant treatment, and recently, we found that juveniles from both "depressed" lines show also increased immobility in this test compared to their controls. We hypothesized that FSL and WKY adolescent rats (about 35 days of age) will display different patterns of social play compared to their controls, as observed after 24 hours of isolation juveniles Frequencies of behaviors were recorded and analyzed during 15-min observations of within-line pairs. Next we examined whether FSL and WKY juveniles have different basal plasma levels of corticosterone and $\mathrm{ACTH}$, compared to their controis. Blood from both lines and form their controls was taken after the rats have been sacrificed. 
FSL juveniles demonstrated significantly higher levels of social play behaviors compared to their controls, while WKY juveniles demonstrated significantly lower levels of social play behaviors compared to their controls.

Significantly higher plasma levels of corticosterone and ACTH werc found in FSL juveniles compared to their controls, while WKY juveniles exhibited significantly lower levels of corticosterone and ACTH compared to their controls.

These results may suggest that both FSL and WKY are genetic animal models of depression in adolescent rats, exhibiting separate patterns of social dysfunction and different abnormality of the hypothalamic- pituitary-adrenal (HPA) axis.

\section{Subdivision of cultured Aplysia neurons into} sub-compartments by spontaneous and localized calcium flashes

Malkinson G. and Spira M.E.Error! Bookmark not defined. Dept. of Neurobiology, Life Sciences Institute. The Hebrew

University of Jerusalem

Recent studies show that the free intracellular calcium concentration $\left(\left[\mathrm{Ca}^{2+}\right]_{i}\right)$ in cultured embryonic neurons is spontaneously and locally elevated with well-characterized frequencies and amplitudes. These spontaneous events ("calcium spikes" or "flashes") are thought to play a role in the differentiation of the neurons and in synaptogenesis (Spitzer, J. Physiol. Paris 96, 2002) and in nerve terminals (Kraus et al., J. of Basic \& Clin. Physiol. \& Pharmacol 2000; 11, 4).

Cultured adult Aplysia neurons, in defined medium, vigorously extend new neurites, form chemical and electrical synapses and reveal short and long term forms of neuroplasticity. Numerous studies of the calcium metabolism in cultured Aplysia neurons revealed that non stimulated neurons maintain the $\left[\mathrm{Ca}^{2+}\right]_{i}$ at steady levels. Here we report however, the detection of significant spontaneous local fluctuations in the $\left[\mathrm{Ca}^{2+}\right]$. The local and transient nature of these spontaneous "calcium-flashes" subdivides the neurons in time and space into calcium-compartments.

For the observations, fluo-4 and rhod-2-pentapotassium were injected into the cultured neurons. Evoked or spontaneous local "calcium-flashes" were imaged by a confocal system. In the main axon and cell body local calcium flashes $\left(\mathrm{F} / \mathrm{F}_{0}=\right.$ 1.5-2) appear at an estimated frequency of 3 per minute. In the branching neurites, the frequency of the flashes is higher, the flashes appear to be generated randomly in space and time, and no quiescent periods are detected. In growth cones produced by axotomy, random local calcium flashes are imaged in the lamellipodium and filopodia. In the growth cone the occurrence of the flashes is at a higher rate.

As in embryonic vertebrate neurons, the Aplysia's calcium flashes are blocked when the normal bathing solution that contains $11 \mathrm{mM}$ calcium is replaced by a calcium free $2 \mathrm{mM}$ EGTA solution. This suggests that the spontaneous initiation of a calcium flash is generated by a calcium influx.

Neuroprotection, neurorescue and neuroplasticity in a cup of tea

Mandel S., Reznichenko L., Tal L., Amit T. and Youdim

${ }^{I}$ Eve Topf and US NPF Centers for Neurodegenerative diseases and Dept .of Pharmacology, Faculty of Medicine.

\section{Technion, Haifa}

Tea intake is commonly associated with traditional beverage rituals and particular lifestyles, especially in Japan, China, India and England. Nevertheless, nowadays it is considered as a source of dietary constituents endowed with biological and pharmacological activities with potential benefits to human health, especially in aging and neurodegenerative diseases. Indeed, accumulating evidence support the hypothesis that oxidative-stress (OS), resulting in reactive oxygen species (ROS) generation and inflammatory processes, trigger a cascade of events leading to apoptotic/necrotic cell death in neurodegenerative disorders, such as Parkinson's (PD) Alzheimer's diseases (AD). Thus, novel therapeutic approaches aimed at neutralization of OS-induced neurotoxicity, support the application of ROS scavengers, transition metals (e.g. iron and copper) chelators and non-vitamin natural antioxidant polyphenols, in monotherapy, or as part of antioxidant cocktail formulation for these diseases. The major polyphenol component of green tea, (-)-epigallocatechin-3-gallate (EGCG) and blueberry extract have been shown to improve age-related cognitive decline and to be neuroprotective in models of PD, $\mathrm{AD}$ and cerebral ischemia/reperfusion injuries. However, recent studies indicate that the radical scavenger property of EGCG is unlikely to be the sole explanation for its neuroprotective capacity. Our investigation into the molecular pharmacological action of EGCG, has clearly shown that this polyphenol acts through a rapid activation of PKC-a and PKC-e in PC-12, SH-SY5Y neuroblastoma cells and mice hippocampus. This results in down regulation of a pro-apoptotic gene cascade involving bax, bad, TRAIL, mdm2 Fas ligand and GADD45. Thus, it is apparent that EGCG has anti-apoptotic (cell survival) activity and may induce $\mathrm{PKC}$-dependent long-term potentiation, resulting in neuronal plasticity. The latter is evidenced by the ability of EGCG rescuing and maintaining viable $\mathrm{PC}-12$ cells, in response to serum neurotrophic factors withdrawal.

\section{Involvement of the plasminogen activator system in} experimental autoimmune encephalomyelitis Maravi-Pinto FY. ${ }^{1}$, Irony-Tur-Sinai $\mathrm{M}$. ${ }^{1}$, Grigoriadis N. ${ }^{3}$ Higazi AE. ${ }^{2}$, Akkawi $S^{2}$ and Brenner T. ${ }^{2}$.

Depts. of Neurology and Biochemistry', Hadassah Medical Center. Jerusalem and Aristotle University. Thessaloniki Greece.

Extracellular proteases such as plasminogen activators (PAs) and matrix metalloproteinases play an important role in axonal and myelin damage. These processes are involved in a variety of CNS disorders such as stroke, encephalomyelitis and multiple sclerosis (MS). In MS, as well as in nerve injury, PAs and their inhibitors are upregulated. Although, it is not clear whether this altered expression is a beneficial or deleterious response of the tissue to the disease. In the present study, we studied the involvement of the PA system in experimental autoimmune encephalomyelitis (EAE), using mice lacking tPA (tPA - $/$ ), uPA (uPA $-/$ ) and UPAR (uPAR - $/-$ ). All the knockout mice tested exhibited severe neurological dysfunction in comparison with the wild type (wt) group. while the tPA -/- mice displayed the most severe clinical symptoms. Histological examination of CNS tissue correlated with the augmented clinical outcome. Increased axonal injury, axonal loss and inflammatory foci were found in the tPA and UPAR $\%$ mice. On the other hand, specific $T$ - cell reactivity towards the encephalitogenic antigen $M O G$ and the
response to $M O G$ presentation by professional antigen presenting cells was markedly reduced in the $\mathrm{KO}$ animals by $40-80 \%$ and $60-80 \%$ respectively. Our results indicate that the PAs system is involved in the axonal and myelin damage occurring during EAE and may be a potential target for treatment of $\mathrm{CNS}$ inflammatory and demyelinating diseases.

\section{Restoration of cerebral blood perfusion by the Ras inhibitor FTS is demonstrated by diffusion weighted imaging in a rat closed head injury model \\ Marciano D. , Shohami E. ${ }^{2}, K \operatorname{loog} Y^{3}{ }^{3}$ and Goelman G. ${ }^{4}$ IDept of Organic Chemistry Israel Institute of Biological Research, Ness Ziona: Depts of Pharmacology and}

${ }^{4}$ Medicinal Biophysics, Hadassah Medical Center, Jerusalem ${ }^{3}$ Dept of Neurobiochemistry. Tel-Aviv University.

Traumatic brain injury (TBI) activates cellular and molecular responses resulting in secondary damages leading to decrease in cerebral blood flow, neuroinflammation and neuronal cell death. A post-traumatic decrease in binding of ligands to NMDAR, indicative of loss of NMDA-receptive neurons was observed. TBl-induced activation of NMDAR caused activation of Ras and its effectors. Previous results showing that inhibiting Ras activation lead to both, a decrease in loss of NMDA-receptive neurons and a decrease in the size of the lesion suggested that the neuroprotective effects of a Ras inhibitor could be also associated with the activated Ras in brain blood vessels. This hypothesis was tested by MRI scanning of post-TBI rat brains, treated with FTS, a synthetic Ras inhibitor. The MRI protocols include diffusion weighted imaging from which apparent diffusion coefficients ( $A D C$ ) maps and semi-quantitative perfusion maps were compiled, and anatomical images from which $\mathrm{T} 2$ maps were compiled. The increase in $\mathrm{T} 2$, the nuclear magnetic resonance transverse relaxation time of water, after trauma is usually attributed to vasogenic edema and infracted areas. The ADC is sensitive to the cytotoxic edema defined as the relative change in the volumes of extracellular versus intracellular spaces triggered by energy failure. MRI distinguishes between the water flowing in capillaries (perfusion), and static water (pure diffusion of intra and extracellular water).

The results analysis shows that there is a measurable therapeutic FTS effect in the brain of rats after TBI: From the day following TBI to one week later, ADC values increase as compared with baseline. In contrast, control rats exhibit a pronounced decrease of ADC values. One week after TBI, T2 values of FTS rat brains return to baseline while in the control rats they are still high. In FTS rats, the perfusion contributes $51 \%$ (1 day) and 26\% (1 week) to the increase in ADC values. In control rats, the decrease of the perfusion contributes $57 \%$ ( 1 ' day) and 79\% ( 1 week) to the decrease of the ADC values. From these results, we infer that the FTS rescue of the brain after TBI can be attributed to an increased cerebral perfusion beside the assumed decrease in Ras-dependent MAPK mediated neuroinflammatory responses to TBI 
Characterization of GRK-2 and $\beta$-arrestin in mood disorders

Matuzany-Ruban A., Schreiber G. and Avissar S. Dept. Pharmacology, Ben Gurion University, Beer Sheva

Mental disorders represent a significant share of disability causes worldwide. The biochemical mechanism underlying the pathophysiology of mood disorders remain unclear. Basic and clinical studies have provided evidence that the serotonin and norepinephrine neurotransmitter systems are involved in the pathophysiology of mood disorders. Biochemical research has focused on the cascade of events involved in the signal transduction mechanisms beyond receptors involving protein-coupled receptors. Alteration in cyclic AMP signaling have been described in depression, bipolar disorder, obsessive-compulsive and schizophrenia. Moreover, chronic administration of antidepressant drugs has been shown to alter this pathway, leading to receptor desensitization and down-regulation of this signal transduction pathway. GRK and - -arrestin are proteins involved in homologous desensitization of receptors following continuous exposure to agonist. This study aims at searching for the possible involvement of GRK-2 and $\beta$-arrestin 1 in the regulatory pathways involved mood disorders. Significant alteration in the immunoreactive levels of both GRK-2 and $\beta$-arrestin 1 were detected in mononuclear leucocytes of patients with mood disorders as compared with normal subject. Patients were examined at untreated-acute state, and following drug treatment that led to mood normalization. These findings support a possible role for GRK-2 and $\beta$-arrestin 1 in the molecular mechanisms underlying the pathogenesis of mood disorders.

Characterization of the neuronal output of the peripheral nervous system of the octopus arm

Matzner H. Sumbre G. Flash T. and Hochner B.

Matzner H. Sumbre G. Flash T.

In previous studies we have shown that the octopus' reaching movement is controlled by a motor program that involves strong feed-forward elements (Gutfreund et al 1998, $J$. Neurosci) and that this program is embedded within the neuromuscular system of the arm itself (Sumbre et al 2001, Science). In the current work we test whether an isolated arm nerve cord can generate a fictive extension motor output - a feature characterizing pure feed-forward control systems. The dorsal axonal tract of a segment, taken from the arm nerve cord, was stimulated and the resulting motor activity was measured from the lateral roots at two different locations down the nerve cord $(\sim 1$ and $2 \mathrm{~cm}$ from the stimulating electrodes). The lateral roots were confirmed as carrying motor activity by recording EPSPs in the muscle fibers that were time-locked to single units recorded en passant in the nerve root. The axonal tract was stimulated with trains of $(50-100 \mathrm{~Hz}, 100-600 \mathrm{~ms})$ stimuli, a paradigm effective in evoking typical arm extensions in denervated or amputated arms (Sumbre et al 2001). Such stimulation evoked a prolonged (up to several seconds) tonic activity in the motor roots and occasionally a burst of activity. The intensity of these bursts declined along the nerve cord. Cross-correlation analysis between the two electrode activities revealed no consistent delay that can match the speed of the traveling EMG recorded in behaving animals (Gutfreund et al 1998). These results suggest that an isolated nerve cord cannot generate patterns of activity that might be implicated with the generation of a feed-forward motor output. On the other hand, we have discovered that the nerve roots conduct afferent activity when muscles are mechanically stimulated, suggesting the existence of a mechano-sensory feedback system. Possibly this sensory feedback is essential for the feed-forward-like propagation of muscle activity along the arm.

Supported by: ISF, BSF and DARPA grants

\section{Glycosaminoglycan in urine of prion infected animals and} humans

Mayer-Sonnenfeld T., Halimi M., Dayan Y., Najjar S., Levy Y. and Gabizon R

Dept. of Neurology; Hadassah University Hospital, Jerusalem Prion diseases are a group of neurodegenerative diseases of animals and humans. Among them diseases such as CJD scrapie and BSE, also known as the mad cow disease. It is well established that the key molecule associated with these diseases and the main component of the prion infectious agent is the aberrant isoform of the normal prion protein, $\mathrm{PrP}^{\mathrm{C}}$ denominated $\mathrm{PrP}^{\mathrm{Sc}}$. However, there are different lines of evidence that connect molecules such as glycosaminoglycans (GAGs) to the metabolism of the PrP isoforms and the pathological alterations observed in brains affected by TSE Since we have found that aberrant isoforms of PrP can be detected in the urine of prion affected animals and humans, we decided to investigate whether there are quantitative or qualitative differences also between the GAGs present in sick individuals as compared to urine samples from normal controls. To this effect, we isolated and analyzed GAGs from appropriate urine samples. Enriched urine samples, normalized for creatinine, were analyzed by well established methods for identifying GAGs such as TLC, agarose- gel electrophoresis and alcian- silver staining of SDS- PAGE gels. In addition, the total amount of GAGs in urine was measured by specific quantitative techniques. Our results show that the total GAG concentration of GAGs in prion urine is significantly higher than in normal controls. In addition, all chromatographic methods used indicate significant qualitative differences between GAGs content of urine from sick, as compared to healthy individuals. These findings are consistent with the presence of an amyloid seed in prion urine, which may be composed of GAGs and PrP, and may incorporate other urine components as well.

\section{Exploring the coordinate system of object related areas using $\mathbf{A M R I}$}

Mckyton A. and Zohary E

Hebrew University, Jerusalem 91904

Perception and action necessitates a transformation from a retinotopic based coordinate system to a world based one. For instance, eye movements result in displacement of a static object's image on the retina, although it remains stationary in the world. On the other hand, tracking a moving object with the eyes results in displacement of the object in the world without concomitant movement on the retina. The receptive fields of neurons in early visual areas (such as V1) are strictly retinotopic. Can a world based representation be found in higher object related visual areas? We classified these cortical regions as world (or at least head based) vs. retinal coordinate based, using the fMRI adaptation technique. This technique is based on the fact that the hemodynamic signal in higher order areas is reduced with repeated presentation of the same visual stimulus. A change in the stimulus position leads to recovery from adaptation. It is unknown, however, whether the recovery is due to the change in the object's retinal position or its absolute (world) position. We observed robust adaptation in the lateral occipital cortex (LO) when the object's position was fixed, although the image of the object on the retina was alternated between the two visual fields. Furthermore, recovery from adaptation was seen when the object moved in the world while keeping its image in the same place on the retina, by similar eye movements. This indicates that unlike the early visual areas, and other object related areas the representation in LO may be specific to the location of objects in the world (or at least taking into account the eye position), irrespective of the position of the object's image on the retina.

The analgesic activity of CB2 selective cannabinoids in noxious, visceral and inflammatory pain models

Meilin S., Weksler A., Richstein A., Berckovitch Y., Avidor B., Yacovan A., Bar-Joseph A. and Fink G.

Pharmos Ltd, Kiryat Weizmann, Rehovot 76326

Accumulated data suggest that cannabinoids are effective in relieving pain. Two cannabinoid receptors have been identified, $\mathrm{CB} 1$ and $\mathrm{CB} 2$. The CB1 receptor is expressed in the nervous system whereas the $\mathrm{CB} 2$ receptor is mainly associated with the immune system. The analgesic activity of PRS-211,096, was previously shown in noxious and inflammatory pain. PRS-211,359 is an ester of PRS-211,096, with a similar affinity for $\mathrm{CB} 2\left(\mathrm{IC}_{50}\right.$ of both compounds $\sim 1$ $n M)$. The analgesic activity of PRS-211,359 was assessed in experimental animal models of noxious and inflammatory pain. The ability of PRS-211,359 to reduce noxious pain was tested using the tail-flick model. ICR mice were injected IP with the compound, vehicle, saline or morphine, and analgesia tested $30,90,150,210$ and 330 minutes later. The analgesic effect of PRS-211,359 was dose dependent, as powerful, but longer lasting than that of morphine $(330 \mathrm{~min}$ with PRS-211,359 vs. $30 \mathrm{~min}$ with morphine) and similar to that seen with PRS-211,096. Inflammatory pain was induced by intraplanar injection of carrageenan in adult male rats after which the animals were injected IP with either vehicle or PRS-211,359. Thermal and mechanical pain threshold was assessed 180 minutes after injection. The difference between the carrageenan and contralateral-treated paws was calculated. PRS-211,359 showed dose dependent analgesia in both thermal and mechanical stimuli, reducing the pain severity by more then $50 \% \quad(p<0.05$ vs. vehicle). Visceral pain was induced by IP injection of $0.6 \%$ acetic acid and analgesia assessed by reduction of writhing over 5 minutes. Pre-treatment with PRS-211,096 dramatically reduced writhing compared with vehicle alone $(0 \pm 0$ vs. $26 \pm 5$ respectively, $\mathrm{p}<0.01$ ) and was far more efficient than known NSAIDs. Thus, PRS-211,359 a CB2 selective cannabinoid is a potential analgesic agent for noxious and inflammatory pain. 
Correlates of auditory streaming in auditory cortex of the awake monkey

Melikyan A., Scheich H., and Brosch M.

Leibniz Institute for Neurobiology, Dept. of Auditory

Learning and Speech, Magdeburg, Germany

Sequences of tones are often perceived as originating from one or different sound sources. A recent study in primary auditory cortex, using sequences of tones of two different frequencies, suggests that the same neurons are excited when the tones are perceived as originating from the same sound source whereas different neurons are excited when the tones are perceived as originating from different sound sources (Fishman et al., Hear Res., 2001:151). The goal of the present study was to extend the search for cortical correlates of auditory streaming by using other tone sequences and including non-primary auditory fields. We prepared a monkey (Macaca fascicularis) for electrophysiological experiments. Multiunit activity was recorded by advancing seven individual microelectrodes into the auditory cortex of the left hemisphere. Stimuli were recycling sequences of two high and two low frequency tones from which we created six different patterns by alternating the order of the tones (high ${ }^{-}-$high $_{j}-$ - $\mathrm{w}_{\mathrm{m}}-$ - $_{\text {ow }}$; high $_{i}-$ low $_{m}-$ high $_{j}-$ low $_{n}$ ). The patterns give rise to different perceptual organizations. Tone duration and intertone intervals were $100 \mathrm{~ms}$ and intensity was about $70 \mathrm{~dB}$ SPL. For each tone we calculated peristimulus time histograms with a bin size of $20 \mathrm{~ms}$ and obtained the bin with the maximal number of spikes. We found that the high-low-high-low type induced statistically significant weaker responses than the high-high-low-low type. The results show that there is more suppression between tones within streams than across streams. This suppression may underlie the processes of streaming.

Exocytosis: Threading of the partial reactions into a functional kinetic mechanism

Mezer A. , Nachliel E. ${ }^{1}$, Gutman M. ${ }^{1}$ and Ashery U. ${ }^{2}$ ${ }^{7}$ The Laser Laboratory for Fast Reactions in Biology, Dept of Biochemistry and ${ }^{2}$ Dept of Neurobiochemistry, Tel Aviv University, Tel Aviv

The exocytosis of a single vesicle is thought to be mediated by a sequence of interactions between cytosolic, vesicular and plasma membrane proteins. In the last decades, the functions of specific proteins in this process have been intensively studied. However the precise sequence of protein-protein interactions that drive the exocytotic process is still a debate and a comprehensive description of protein-protein interaction, based on chemical kinetics had not been attempted. In the present study, the reactions between the synaptic proteins were transformed into a set of coupled, non-linear ordinary differential equations where the rate constants and some of the proteins concentrations are adjustable parameters. Recent studies provided some of these parameters, while a search in the parameters space for the others led to accurate reconstruction of the basic $\mathrm{Ca}^{2+}$-driven vesicular fusion. In addition, using the model we were able to reconstruct complex experimental protocols as a sequence of stimulations and munc1 3 overexpression. The model can also give an indication of the dynamic changes of different intermediate complexes during exocytosis. For example it allows identification of the empirically defined 'Readily Releasable' and the 'Slowly Releasable' pools of vesicles with precise synaptic protein complexes formed during the maturation process. The mode allows postulation and prediction of different scenarios regarding deletion and manipulation of some proteins involved in the exocytosis process. The strength of the present model is that we can easily implement another intermediate step according to newly available data about the concentration of specific protein or information about binding kinetics. Therefore, we have created for the first time a comprehensive kinetic model describing the dynamics of interaction between key synaptic-proteins that are associated with exocytosis.

Brain activity intensity location during orthographic and phonological processing using the LORETA program: Comparison between adult dyslexic and regular readers Miller-Shaul S. and Breznitz Z.

Laboratory for Neurocognitive Research, Brain and Behavior Center University of Haifa

Consensus exists among different dyslexia researchers that the origin of the dyslexia deficit is not homorganic. In an attempt to investigate this issue recent studies are investigating the brain activation of dyslexics as compared to regular readers when performing various reading tasks. This study focuses on examining the intensity and the location of activity in the brain while performing orthographic and phonological tasks by using the LORETA (Pascual-Marqui et al:, 1994) program. The study included 40 subjects all university students: 20 developmental dyslexics and 20 chronological age matched regular readers.
Phonological and orthographic processing was examined by using lexical decision tasks. Behavioral and electrophysiological measures were used with evoked potential methodology (ERP). The EEG records of the experimental tasks were analyzed with the LORETA program tasks were analyzed 1994

The findings confirm the hypotheses concerning differences between the groups at both points in time, P200 and P300, for orthographic and phonological processing. Differences were also found between tasks within each group at both points in time.

Results of the study contribute to our understanding the differences in brain activity between phonological and orthographic processing as well as contribute to our understanding the origin of dyslexia phenomenon.

Minimal traumatic brain injury produces cognitive deficits and behavioral disturbances in mice

Milman A. Weizman R., ${ }^{2,3}$ and Pick C.G.

${ }^{7}$ Dept. of Anatomy and Anthropology, and ${ }^{2}$ Psychiatry

Tel-Aviv University Sackler School of Medicine, Israel.

Tel-Aviv Community Mental Health Center, Tel-Aviv, Israel.

Background: Victims of mild traumatic brain injury (mTBI) do not show clear morphological brain defects, but frequently suffer lasting cognitive deficits, emotional difficulties and behavioral disturbances.

Objective: To investigate the effects of mild traumatic brain injury on cognition and depression in mice.

Methods: Experimental mTBI was produced using the concussive head trauma device. Different groups of mice were tested 7,30,60 and 90 days post-injury for cognitive function in the swim T-maze and the passive avoidance test, and for depression in the forced swimming test.

Results: There were significant differences in behavior between the mTBI mice compared to the controls in the swim T-maze and passive avoidance test, beginning 30 days post-injury. There was a significant difference in the depressive behavior between the mTBI mice and the controls, which showed a depressive state in the injured animals beginning 7 days post-injury.

Conclusion: Mild traumatic brain injury results in a cognitive impairment starting a month after the injury, and a depressive state a week after the injury. These results demonstrate that persistent deficits of cognitive learning abilities and behavio in mice, similar to those observed in human post-concussive syndrome, can follow mTBI

In vivo imaging of dendritic spines in the hippocampus Mizrahi A. Crowley J.C. Shtoyerman E., and Katz L.C. Howard Hughes Medical Inst. and Dept. of Neurobiol., Duke University Medical Center, Durham, NC, 27710, USA

The hippocampus plays critical role in learning and memory but its deep anatomical locale makes it technically difficult to image in the intact brain. In order to image the hippocampus, we have developed a surgical preparation that allows imaging of dendritic stucture in the adult mouse hippocampus using a conventional 2-photon microscope (Zeiss LSM 510). When applied to transgenic mice constitutively expressing GFP in a subset of pyramidal cells, this technique enabled visualization of CAl dendrites and spines in exquisite detail in vivo Individual spines were readily resolved on both basal and apical dendrites, and could be followed over extended periods.

Hippocampal dendrites of adult mice were imaged for at least 4 hours ( $n=1082$ spines; $N=4$ mice). To this end, we find a high degree of structural stability over the 4-hour time window as the vast majority ( $>99 \%$ ) of the spines remained stable. We are currently assessing structural plasticity during various experimental manipulations of electrical activity.

Thus, using this preparation, it is now possible to directly image hippocampal dendrites and spines. In addition, this method may prove useful to study plasticity in the hippocampus using other imaging techniques.

Auditory evoked potentials and behavioral measures among dyslexic students in response to linguistic stimul discriminated by temporal cues

Moisesco-Yiflach T ${ }^{1}$, Lamm $\mathrm{O}^{2}$ and Pratt $\mathrm{H}^{1}$

Evoked Potentials Laboratory, Technion, Haifa; ${ }^{2}$ School Of

Education, University of Haifa

Introduction: Students with phonologiclauditory dyslexia present high intelligence and normal attentional and visual skills. Some researchers suggest a specific impairment in processing the temporal aspect of auditory information.

The discrimination of voice/voiceless syllables relies mainly on the perception of the time differences between the beginning of the vocal cords vibration and the release of the stop in the oral cavity - a temporal cue (Voice Onset Time VOT). 
Objectives: To explore the brain activity evoked by linguistic stimuli that rely on temporal cues among dyslectics with years of reading rehabilitation and high academic achievements were studied. Event-Related Potentials (ERPs) manifestations of processing, beginning with the early obligatory components, through the late components and behavioral overt responses were examined

Methods: Fourteen dyslectic university students and 14 normal reading students were tested. The subjects performed an active discrimination task (Oddball paradigm). Stimuli: pair of synthetic $/ \mathrm{ba} / / \mathrm{pa} /$ monosyllables with a voice onset time difference of $20 \mathrm{msec}$. The ERPs were recorded from 21 electrodes. In addition to waveform analysis, LORETA source current density estimation was performed.

Results: Although no significant differences were found in the behavioral measures (reaction time, accuracy), ERP components revealed significant differences between subject groups. Differences were found from the early component arising from the auditory cortex (N100) via P2 and as late as P300 that reflects the conscious perception of the stimuli. The most consistent and significant difference was a prolonged latency of all the ERP components in the dyslectic group. There were also differences in the scalp distribution of the potentials. The source current density distributions showed group differences in the brain structures involved and the time course of their activation. In the control group the typical early activity (around N100) was characterized by rapid alternations of maximum activity between the temporal lobes of the two hemispheres. The late activity (around P300) was hemisphere. Among the dyslectics the early activity did not show rapid changes between the two hemispheres, and the late activity was mainly in the right temporal lobe

Conclusions: Phonologic dyslectic students process temporal cues of linguistic stimuli differently than normally reading controls. The differences manifest in the speed of processing and in the brain structures involved.

\section{Storage capacity of WM in a network of spiking neurons} with randomly chosen stimuli

Mongillo G. Curti E. and Amit DJ.

INFM dip. di Fisica Universita' di Roma, La Sapienza; Racah Institute of Physics, Hebrew University, Jerusalem dip di Fisiologia Umana, Universita' di Roma, La Sapienza

We calculate, in Mean-field the storage capacity of working memory of realistic networks of excitatory and inhibitory spiking neurons. The stimuli embedded in the synaptic matrix, of two-state synapses, are with a coding level \$ \$, are chosen at random (hence with overlaps) and the synaptic matrix is formed by a slow plasticity, upon repeated presentation of the set of stimuli to be memorized.

Working memory capacity is the maximal size of the training set, for which the network can maintain selective delay activity states.

The results, obtained in MF-theory are compared with simulations of microscopic networks of spiking neurons, monitoring the average rates in neural population samples, correlations of visual response distribution to delay activity distribution and rate histograms across the memories.

The storage capacity is studied as a function of the ratio of 1 . the high-to-low synaptic efficacy; 2. the efficacy of inhibitory to excitatory efficacy; 3 . the of the ratio of depression-to-potentiation probability in learning; 4 . of the coding level.

Supported by the Israel Science Foundation (ISF) Grant $\$ 8009$, and the Center of Excellence Grant 'Statistical Mechanics and Complexity" of the INFM, Roma-1.

\section{Can physiological strain index (PSI) reflect central thermoregulatory activity?}

Moran D.S.

Heller Institute of Medical Research, Sheba Medical Center, Tel-Hashomer, Sackler Faculty of Medicine, Tel Aviv University, Tel Aviv

Upon subjection to heat stress, the thermoregulatory center, located in the preoptic area/anterior hypothalamus activates two major heat defense effectorial loops in a hierarchical manner: vasodilatation followed by sweating. Non-invasively, the temperature thresholds (Tsh) for activation of these physiological responses reflect central thermoregulatory activity. To assess whether Tsh is also indicative of the heat strain developed upon subjection to heat stress, in this presentation differences in peripheral vasodilatation Tsh (VTsh) in three groups of exercising human subjects differing in their heat tolerance (heat tolerant, heat intolerant and heat acclimated) was correlated with the calculated PSI strain index (based on rectal temperature (Tre) and heart rate (HR) online recordings, Moran et al., Am J Physiol. 1998 275:RI 29-34.) upon subjection to exercising in hot environment. The onset of vasodilatation and VTsh of the non-tolerant group was earlier and at a higher Tre $\left(37.55^{\circ} \mathrm{IC}\right.$ compared to tolerant and heat acclimated subjects (37.17'IC and 37.01'IC, respectively) There was a correlation between the onset for a developed strain and VTsh in the non-heat tolerant group. This was not the case in the heat acclimated and the heat tolerant groups. In the later. VTsh lagged behind the onset of the developed strain, suggesting that although PSI is indicative of the developed strain its multi-factorial dependence make it unsuitable as a measure of central thermoregulatory activity.

Enhanced temporal non-linearities in human

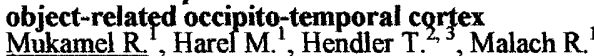
${ }^{T}$ Dept. of Neurobiology, Weizmann Institute of Science, Rehovot $76100 ;{ }^{2}$ Functional Brain Imaging unit Tel Aviv Sourasky Medical Center, Tel Aviv 64239; 3 Faculty of

Medicine, Tel Aviv University, Tel Aviv 69978

To what extent does neural activation in human visual cortex follow the temporal dynamics of the optical retinal stimulus? Specifically, to what extent does stimulus evoked neural activation persist after stimulus termination? In the present study, we used functional Magnetic Resonance Imaging (fMRI) to explore the resulting temporal non-linearities across the entire constellation of human visual areas. Gray-scale images of animals, houses, and faces were presented at two different presentation rates- 1 and $4 \mathrm{~Hz}$ and fMRI signal was analyzed in retinotopic cortex, area MT/V5, and in high order occipito-temporal visual areas. In early visual areas and the motion sensitive area MT/V5, a four-fold increase in stimulus presentation rate evoked a two-fold increase in signal amplitude. However, in high order visual areas, signal amplitude increased only by $25 \%$. A control experiment ruled out the possibility that this difference was due to signal saturation ("ceiling") effects. A likely explanation for the stronger non-linearities in occipito-temporal cortex is a persistent neuronal activation that continues well after stimulus termination in the $1 \mathrm{~Hz}$ condition. These persistent activations might serve as a short term (iconic) memory mechanism for preserving a trace of the stimulus even in its absence and for future integration with temporally correlated stimuli

Effort related changes in responses to tone pairs: An MEG study

Nahum $\mathrm{M}^{1,2}{ }^{1,}$, Renvall $\mathrm{H}^{1}{ }^{1}$, Hari $\mathrm{R}^{1}{ }^{1}$ and Ahissar M. ${ }^{2}$

${ }^{7}$ Brain Research Unit, LTL, Helsinki University of Technology, Espoo, Finland; ${ }^{2}$ ICNC, Hebrew University, Jerusalem,

Israel

When sounds are presented in pairs with intervals of less than $300 \mathrm{~ms}$, the $100-\mathrm{ms}$ cortical response $(\mathrm{N} 100 \mathrm{~m})$ to the $2^{\text {n }}$ stimulus is enhanced compared to the $1^{\text {st }}$ (Loveless et al., 1989 1996). We studied the build-up of this enhancement effect and its relationship to task difficulty.

Whole-scalp magnetoencephalographic (MEG) signals were recorded from 10 subjects with a 306-channel neuromagnetometer. Sequences of 5 pairs of $50-\mathrm{ms}$ binaural tones were presented with stimulus-onset asynchronies (SOA) of $150 \mathrm{~ms}$, inter-pair intervals (IPI) of $1 \mathrm{sec}$ and an inter-sequence-interval of 10 secs. Subjects were asked to discriminate inter-pair frequencies under two difficulty conditions: easy $(1000$ vs. $1100 \mathrm{~Hz})$ and difficult $(1040 \mathrm{vs}$. $1055 \mathrm{~Hz}$ ). In $50 \%$ of the pairs the tones were of the same frequency.

All stimuli elicited prominent $\mathrm{N} 100 \mathrm{~m}$ responses in the auditory cortices, peaking at $83 \pm 3 \mathrm{~ms}$ to the $1^{\mathrm{st}}$ tone and at $130 \pm 17$ $\mathrm{ms}$ to the $2^{\text {nd }}$ tone of each pair. Response to the $1^{\text {st }}$ tone in a pair decreased by $52 \pm 3 \%$ from $1^{\text {st }}$ to the following pairs. In contrast, response to the $2^{\text {nd }}$ tone in a pair gradually increased from the $2^{\text {nd }}$ to the $5^{\text {th }}$ pair. Consequently, the ratio between the $2^{\text {nd }} \& 1^{\text {st }}$ responses in each pair increased throughout the pairs
from $0.53 \pm 0.03$ to $1.22 \pm 0.04(p<0.001)$. Task difficulty did not affect response amplitudes, whereas peak latencies of the $2^{\text {nd }}$ response in each pair were $11 \pm 4 \mathrm{~ms}$ longer for the difficult condition (Both hemispheres: $p<0.03$ ). An even greater difference was found in the offset of the slow shift (SS) of the response, with the $2^{\text {nd }}$ response lasting $93 \pm 26 \mathrm{~ms}$ longer $(p<0.002)$ under the difficult condition. Preliminary results suggest that the source of this "effort related" difference lies in the supratemporal auditory cortex

Taken together our findings suggest that the difficulty of frequency discrimination can affect the $N 100 \mathrm{~m}$ response and is related to the dynamics of response build-up rather than to response strength as such. 
The effect of pre-challenge learning on MK801 induced psychosis-like behavior in an animal model of schizophrenia

Naimark A. Barkai E. and Cohen $\mathrm{H}$

Ben Gurion University of the Negev, Beer Sheva

This study aimed to examine whether pre-challenge learning prevents psychosis-like behavior in an animal model of schizophrenia, and to describe the leaning-related cellular mechanisms which attenuate the course of schizophrenia. By combining behavioral studies with cellular neurophysiology/morphology we explored how learning protects against the psychotomimetic effects of the NMDA receptor antagonist MK801.

Rats were trained to distinguish between pairs of odors in an olfactory discrimination task. We examined whether such olfactory-learning induces protection against the effects of ip injections of $0.1 \mathrm{mg} / \mathrm{kg} \mathrm{MK801} \mathrm{in} \mathrm{a} \mathrm{series} \mathrm{of} \mathrm{behavioral} \mathrm{tasks} \mathrm{-}$ the Morris water maze, prepulse inhibition and elevated plus maze MK801 caused sensorimotor disturbances spatial learning acquisition deficit, and swimming strategy alterations in pseudo trained and nałve rats, but caused neither in trained rats.

Brain slices of the hippocampus were prepared and intracellular recordings were obtained from hippocampal CA1 pyramidal neurons. Data show paired pulse facilitation is reduced in neurons from trained rats indicating enhanced synaptic transmission between $\mathrm{CA} 3$ and $\mathrm{CA} 1$ neurons.

Learning-induced protection may occur due to changes that take place in synapses connecting CA3 with CA1 hippocampal neurons, as a result of strengthening of the non-NMDA mediated synaptic transmission. Thus, AMPA-receptor mediated synaptic connectivity is enhanced after learning in a manner that allows the hippocampal network to maintain its function even in the absence of NMDA-receptor mediated activity.

Planning of bimanual movements in primary motor cortex Nathan C., Paz R., Boraud T., Bergman H. and Vaadia E. Dept. of Physiology, Hadassah Med. School and The Interdisciplinary center for Neural Computation, Hebrew Interdisciplinary center
University, Jerusalem

Recent findings defy the classical view of the primary motor cortex (M1) as controlling only for contralateral movements. Bilateral related activity, i.e. significant modulation in relation to both ipsilateral and contralateral unimanual movements, was found in high densities in Ml as in 'higher' motor areas (SMA, CMA, PMd and PPC).

We have previously shown that a large proportion of neurons in the primary motor cortex exhibit bimanual related activity as well. Such activity is defined as one that cannot be explained from the activities associated with the two uni-movements that comprise the bimanual movement.

We extend here these results by showing that bimanual related activity occurs not only during the movement itself but also during movement preparation and even when the movement cannot yet be fully planned. Monkeys were trained to perform 8 directions center-out task, either uni-left, uni-right or bimanual. Each trial started with a laterality signal (i.e. indicating uni or bi movement) followed by a directional signal and only then a go signal. We found that a large proportion of neurons in the primary motor cortex respond to Laterality cue , and a unique combined population-rate response clearly differentiate between the three of upcoming movements as well. moreover, we show that percentage of bimanually related neurons increases along the trial performance.

The results strengthen other recent findings showing that $M 1$ participates in planning for complex movements and particularly in planning and executing coordinated movements of the two arms.

\section{Characterizing secondary degeneration and protective} autoimmunity kinetics by diffusion weighted MRI Nevo U.,2, Molshanski-mor $S_{1}^{1}$, Hauben E. , Akselrod S. ${ }^{2}$, Neeman ${ }^{1}$ and Schwartz $M$

Weizmann Institute of Science, Rehovot; ${ }^{2}$ Tel Aviv University. Tel Aviv

Secondary degeneration following spinal cord injury (SCI) is a self-perpetuating process that contributes to the loss of neuronal cell bodies and axonal fibers. Our group has pioneered the use of Autoimmune Modulation to protect the CNS from secondary degeneration (Moalem G. et al. Nat Med 1999). This approach involves passive or active immunization with self-antigens residing in the site of the lesion. We have lately suggested 'comprehensive immunity' (Nevo U. et al. Trends in Mol. Med), a possible physiological immune based neuroprotection mechanism whereby the protection involves a transient phase of tissue loss.
In this work we use the Diffusion weighted MRI (DW-MRI) technique to characterize the dynamics of degeneration in rats that were subjected to $\mathrm{SCl}$. We further use this technique to test the predictions suggested by our 'comprehensive immunity' approach. We vaccinated Male adult Lewis rats with copolymer that boosts the protective autoimmunity. At four time-points after the injury, rats were sacrificed and their excised spinal cords were scanned by DW-MRI (Nevo U. et al. Magn Reson Med. 2001). Images were then analyzed to yield the values of the Apparent Diffusion Coefficient, and the Anisotropy to displacement of water molecules. Values of these parameters where then averages across slices, and normalized by the slices area.

Our result quantified the kinetics of the degenerative process, and showed a transient phase in which the tissue loss in the vaccinated animals exceeded the loss seen in the control group. Nevertheless, the overall recovery, when the two groups reached a plateau was better in the vaccinated than in the control group.

Munc18 phosphorylation enhances vesicle pool refilling. $\mathrm{Nili} \mathrm{U}^{1}$, De Vit H. ${ }^{2}$, Tonnen R. ${ }^{2}$, Verhage M. ${ }^{2}$ and Ashery. ${ }^{1}$ ${ }^{T}$ Dep. Neurobiochemistry, Tel Aviv University, Israel and ${ }^{2}$ Dep.of Functional Genomics, Vrije Universiteit, Amsterdam, The Netherlands

During repetitive or intense stimulation, neurosecretory cells and neurons undergo use-dependent changes in secretory strength which are partially mediated by activation of protein kinase $C$ (PKC). However, the molecular targets of PKC and their mode of action remain elusive. In vitro studies have demonstrated that Munc 18 can be phosphorylated by PKC in a calcium-dependent manner and that this phosphorylation inhibits its interaction with Syntaxin. In the present study we investigated the effect of Munc18 phosphorylation on catecholamine secretion from chromaffin cells by using membrane capacitance measurements following flash photolysis of caged calcium. In our experiments we used a protocol consisting of two flash stimulations given two minutes apart. The response to the second flash stimulation assesses the ability of the cells to refill their releasable vesicle pools, which are depleted following the first flash stimulation. Overexpression of wild-type Muncl 8 caused an increase in the secretory response to both the first and second flash stimulations, as compared to control cells. In contrast, a Muncl8 mutant (Munc18-3A), that mimics the non-phosphorylated form of the native protein, caused an increase in response to the first, but not the second flash stimulation. Preliminary results with a Munc18 mutant (Munc 18-D), that mimics a constitutively phosphorylated form of the native protein, showed a robust increase in the response to both the first and second flash stimulations. These results suggest that Munc18 enhances the secretory response to the first flash in a phosphorylation-independent manner, and contributes to vesicle pool refilling in a phosphorylation dependent manner. As was shown recently, following the first flash stimulation PKC is activated by calcium and acts on specific targets to enhance vesicle pool refilling. Our data suggests that munc18 is one of those targets.

High amplitude, visual-like activations in human visual cortex during the complete absence of visual perception:

Implications for visual awareness

${ }^{N}$ School of Computer Science, Tel Aviv University Tel Aviv, Tel Aviv $61390 ;{ }^{2}$ Dept. of Neurobiology, Weizmann Institute of Science, Rehovot 76100; ${ }^{3}$ The Interdisciplinary Center for Neural Computation, Hebrew University, Jerusalem

A fundamental question in human brain research is the nature of the neural correlate of visual awareness. Recent studies using single-unit recording in the alert monkey, as well as functional MRI in human subjects suggested that neuronal activity in high order visual areas could be the neural correlate of visual awareness. We have found that during complete darkness, well known visual areas show high amplitude IMRI activations, with strong peaks occurring at comparable amplitudes to those found during vivid visual perception. This finding demonstrates that strong activity levels in high order visual cortex are not sufficient for visual awareness. The parameter that distinguished the activity during darkness from visually-driven activations was spatial de-coherence, i.e. the breakdown of correlated activations. Thus, during darkness, functionally distinct object areas were concurrently active, but this activity became rapidly and significantly de-correlated $(39 \%$ drop in correlation level, $p<0.008)$ during visual stimulation. The darkness activations showed consistent neuro-anatomical distribution which ruled out purely hemodynamic or scanner noise sources. Control experiments provide initial insights about the possible mechanisms 
underlying the darkness activity. These experiments argue against visual imagery or processes which enhance EEG alpha band power as sources of the coherent darkness activity. We conclude that spatial de-coherence of cortical activity patterns rather than activity per-se plays a critical role in conscious visual awareness.

Supported by the AMN foundation, the Academy of Science Center of Excellence for Applied Geometry, and grant 8009

\section{Effects of a membrane permeable metal chelator on} zinc-regulating proteins in mice

Nitzan Y.B. , Elgazar V. 1 , Sekler $I^{2}{ }^{2}$, Hershfinkel M. ${ }^{1}$ and Silverman W.F.

Depts ${ }^{I}$ Morphology and Physiology, Zlotowski Center for Neuroscience, Ben-Gurion University of the Negev, Beer Sheva 84105 Israel

Recent evidence for the involvement of zinc in the formation of $\square$-amyloid plaques in the brain in Alzheimer's disease (AD) has led to the establishment of new therapeutic strategies for the disorder based on metal chelation. We measured the degree of free zinc depletion in CD-1 mice treated with clioquinol (CQ), an antibiotic and membrane-permeable chelator, recently reported as a potential treatment for Alzheimer's disease (Cherny et al., 2001). The effect of CQ was examined using the fluorescent zinc probe, TSQ. Mice injected intraperitoneally (i.p.) with CQ showed a dramatic reduction in chelatable zinc in the three organs examined, brain, testis and pancreas. In contrast, mice given $\mathrm{CQ}$ orally, showed no significant difference in levels of chelatable zinc in those same tissues. Chelation of brain $\mathrm{Zn}^{2+}$ by ip. $\mathrm{CQ}$ lasted less than 5 hours. Using dual-label immunofluorescence and confocal microscopy, we next examined the effects of $C Q$ administered i.p. on expression of $\mathrm{ZnT}-1$ and metallothionein (MT-I/II), in olfactory bulb (OFB), hippocampus and cerebellum. In the CNS, MT-I/II is expressed exclusively in non-neuronal cells, in contrast to $\mathrm{ZnT}-1$ (Sekler et al., J. Comp. Neurol. $447: 201-209,2002$ ). In hippocampus $C Q$ had no effect on $\mathrm{ZnT}-1$ and MT-I/II expression. In contrast, ZnT-I demonstrated a significant reduction, and $\mathrm{MT}-\mathrm{I} / \mathrm{II}$ was virtually absent in both $\mathrm{OFB}$ and cerebellum following $\mathrm{CQ}$ treatment. In hippocampus, zinc stores recovered rapidly from the effects of CQ, with synaptic zinc, achieving control levels by $5 \mathrm{hrs}$ after administration of the chelator. However, in the cerebellum, where chelatable zinc stores are negligible under control conditions, chelation had a significant effect on expression of zinc-regulating proteins. This study demonstrates that in the mouse, $C Q$ administered orally, does not significantly alter levels of chelatable zinc. On the other hand, administration of $C Q$ via other routes may be useful in the context of zinc-mediated cell injury.

\section{Localization versus configuration categorization in rapid} perception

Nizan A., Shneor E. and Hochstein S.

Neurobiology Dept., Institute of Life Sciences and Neural

Computation Center, Hebrew University, Jerusalem

Subjects detect rapidly an odd element which differs from its surroundings in a single dimension, but they cannot necessarily report its specific location. Treisman \& Gelade (1980) found that only with the help of focused attention can subjects both identify and localize an odd element. On the other hand, Sagi \& Julesz (1985) found that detection and localization are two parallel fast and early processes, that do not demand focused attention. Reverse Hierarchy Theory (RHT; Ahissar \& Hochstein 1997, 2002) predicts that early, implicit processing leads to explicit detection at high cortical levels, while later explicit processing adds details such as precise localization, by guiding attention to appropriate low cortical level mechanisms. We replicated the Sagi and Julesz experiment, and tested subject localization ability. We presented a round array of 36 while diagonal line elements with 2 or 3 elements replaced by a horizontal line and asked subjects $(n=6)$ to detect how many odd elements were in the array. In another experiment, with 3 odd elements, we asked subjects to categorize the configuration as regular or special, i.e. an isosceles triangle. The difference between these two triangles, as in the Sagi and Julesz experiment, was the position of one triangle corner by 1 place in the array, which is why they called subjects' distinguishing between the two triangles, a localization task. However, in a separate experiment, the same subjects were asked to directly localize the specific place of one odd element, which appeared in a similar array. Our results showed good performance in the detection and categorization tasks, but poor performance in the direct localization task. Therefore, we reinterpret the Sagi and Julesz result and suggest that triangle categorization is a holistic, high-level task, rather than a low-level localization task. We conclude that detection and localization are significantly different from each other. Detection (as categorization) is an easy, early, high cortical level task, while localization is a harder task, that takes place at low cortical levels, where the receptive fields are small. Thus, the specific position of an odd element can be detected only with the help of focused attention. Our results are consistent with RHT and the notion of a holistic view of the visual scene.

Supported by an Israel Science Foundation "Center of Excellence" grant and the US-Israel Binational Science Foundation.

Bifunctional compounds eliciting both anti inflammatory and cholinergic activity as potential drugs for CNS

disorders ${ }_{\text {Nizri E. }}$, Adani R. ${ }^{2}$, Meshulam $\mathrm{H}^{2}{ }^{2}$, Amitai G. ${ }^{2}$, and Brenner $T^{1}$

I Laboratory of Neuroimmunology, Dept. of Neurology, Hadassah HebrewUniversity Medical Center, Jerusalem, ${ }^{2}$ Division of Medicinal Chemistry, IIBR, Ness Ziona.

The existence of cholinergic receptors in cells of the immune system is well documented. Recently, it was found that the immune cholinergic system plays a regulatory role in inflammatory processes. These findings prompted us to test the efficacy of IBU-PO, a bifunctional compound containing both non-steroidal anti inflammatory drug (NSAID) ibuprofen (IBU) and a cholinesterase inhibitor Pyridostigmine Octyl (PO) as cholinergic up-regulator (CURE). IBU-PO was examined in experimental autoimmune encephalomyelitis (EAE) an autoimmune model in mice for CNS inflammation. IBU-PO ameliorated significantly $(40-50 \%)$ the clinical score in EAE, when given recurrently during the course of the disease ( $1 \mathrm{mg} / \mathrm{kg}$, ip, during 30 days). The observed clinical improvement was further supported by ex vivo results: T cell taken from the treated animals showed decreased proliferation in response to various mitogens (e.g. PHA) and also decreased proliferation to MOG, indicating reduced responsiveness of MOG specific $T$ - cells. In vitro experiments done in human-borne lymphocytes were in consistence with the animal results. In addition, IBU-PO down regulated nitric oxide (NO) production in cultured rat brain asrtocytes. In an attempt to determine which moiety is responsible for the observed effect, we tested the two components IBU and PO separately. The T cell proliferation response was partially inhibited by both IBU and PO, but the combined effect of IBU-PO was more enhanced. Our findings indicate that combining the anti-inflammatory activity of NSAID with cholinergic intervention may be a novel approach for the treatment of CNS inflammation and demyelinating disorders such as Multiple Sclerosis.

Unique prolonged brain activity to verbal stimuli with negative subjective meaning revealed by electrophysiological functional brain imaging

Ofek E. and Pratt $\mathrm{H}$.

Evoked Potentials Laboratory, Technion - Israel Institute of Technology, Haifa

Introduction: The effects of subjectively negative content of verbal stimuli on brain activity have not yet been demonstrated. Verbal stimuli in general have multiple semantic dimensions (e.g., meaning, affective valence), complicating the study of neural correlates of semantic content In contrast, first names have only one major semantic dimension (subjective valence). The subjective meaning of a first name is mostly acquired from familiar people in the subjects' life that have this name. Specific brain activity to subjectively cmotionally loaded stimuli has already been demonstrated. The purpose of this study was to indicate the unique brain activity to names with a negative affective valence - names of persons who have hurt the subject in the past. Objective: Identify whether a unique brain activity pattern is evoked by stimuli with subjectively negative valence.

Methods: EEG was recorded while twelve right handed subiects performed a task of identifying 3 emotionally neutral names that ended in a specific consonant from a list of 25-30 names. Names were auditory presented. Following the experiment, subjects were interviewed and the names were rated for their valence. For each subject, the name of the person who hurt the subject the most was chosen. Event-Related Potentials (ERPs) were averaged separately for each name, for each subject. The time course of brain activity to names with subjectively negative valence compared to neutral names was derived using the LORETA method.

Results: Enhanced activation to stimuli with subjectively negative valence lasted from $200 \mathrm{msec}$ until at least $950 \mathrm{msec}$ after stimulus onset. Activity involved mainly the left hemisphere, and had a unique time course, involving several cortical areas at different time periods: Broca and Wernicke areas, secondary auditory cortex, prefrontal cortex, posterior cingulate gyrus, parahippocampal gyrus, postcentral gyrus, and cuneus. 
Conclusions: Names of people who have hurt the subject in the past evoke a unique and prolonged brain response. The response includes a specific time course of activation in language related areas, secondary auditory areas and limbic system. The left hemisphere dominates the response.

\section{Neutralization of apoE4 phenotypes with apoE4 specific} antibodies

Ophir G., Smorodinski NI, Dolev I. and Michaelson DM. Dept. of neurobiochmisrty, faculty of life scinces, Tel-aviv university, Tel Aviv

The apoE4 isoform of human apolipoprotein $E$ is associated with several brain pathologies, including Alzheimer's Disease (AD), multiple sclerosis, stroke and recovery from head injury. Animal models, in which mice transgenic to human apoE4 and apoE3 (which is the AD benign isoform) were subjected to distinct experimental paradigms, revealed that apoE4 is associated with dominant gain of negative effects specific to this isoform. Although several mechanisms have been proposed for these pathological effects of apoE4, their specific roles in mediating the effects of apoE4 in human diseases remaines to be determined. Accordingly, a theraputic approach directed at the neutralization of apoE4 is expected to be effective for patients with the apoE3/E4 genotype, which are the vast majority of the apoE4 isoform carriers.

In the present study, we prepared and characterized monoclonal antibodies (mAbs), which bind apoE4 isoform-specifically and tested their capabilities to neutralize apoE4 related brain phenotypes. mAbs were prepared by immunizing mice with an apoE4 specific peptide, and screened for their ability to interact specifically with apoE4 and not apoE3 in immublot, immunopercipitation and immunohistochmical assays. The biological effectivity of these antibodies was initialiy assessed by measurments of their ability to neutralize apoE4 dependent Ax deposition. This was preformed by a chronic i.c.v administration of Thiorphan (an inhibitor of the Ax degrading enzyme - neprilysin) which results in enhaced brain Ax deposits in apoE4 transgenic mice compared to apoE3, apoE $\mathrm{KO}$ and control mice. This experiment revealed that chronic i.c.v administration of the specific anti apoE4 mAbs neutrilized the apoE4 dependent enhancment of $A i$ deposition. The extent to which apoE4 specific behavioural traits can also be revesed by the anti apoE $4 \mathrm{mAbs}$ is currently being investigated.

Serial memory in monkeys: Is encoding of ordinal category numerical (absolute) or analog (relative)?

Orlov T. ${ }^{1}$, Amit DJ. ${ }^{2}$, , Zohary $\mathrm{E}^{1}$ and Hochstein $\mathrm{S}$.

${ }^{T}$ Int. of Life Sciences. Cent. for Neural Computation, and ${ }^{2}$ Int. of Physics, Hebrew University, Jerusalem, 'Universitx di

Roma La Sapienza, Rome

Monkeys use a combination of mnemonic strategies to retrieve lists of ordered images (Orlov et al., Nature, 2000; Cerebral Cortex, 2002). We presented them with thirty images, divided into 10 triplets, repeatedly in fixed temporal order. On each trial three images were shown sequentially, followed by a test stimulus consisting of the three images and a distractor. The monkeys touched the three images in their order of prior presentation. Surprisingly, their dominant mnemonic strategy was categorization of the images according to their ordinal position. We now ask whether this position is encoded in absolute or relative terms? If encoding is absolute (numerical), monkeys actually label images as 1st, 2nd, etc. If it is relative the image ordinal position can be categorized relatively as initial, intermediate and last. To examine this issue we trained two monkeys on retrieval of the item order in triplets and quadruplets of images, and tested them on a two-set test. Each trial of the test consisted of four images presented simultaneously: three of them belonged to the set of triplets and one was from the set of quadruplets (as a distractor). The monkeys were rewarded for touching in correct order the images from the triplet set. If encoding is absolute, they are expected to err by touching the distractor when it belongs to the same numerical category as the correct image (e.g. touching a distractor from the 3 rd category during the 3 rd touch). If encoding is relative, they would confuse between correct and distractor images if they belong to the same relative (last) category (touching distractor from the 4th category for the 3 rd touch). Results show that monkeys mainly err if the distractor and correct image are from the same numerical category. We conclude that encoding of ordinal number categories is numerical for short item lists. Supp. by Center of Excellence grant (the Israel Science Foundation) and grant from the National Institute for Psychobiology
Conceptual priming of ordinal number category

Orlov T., Haroush K. Zohary E. and Hochstein S.

Dept. of Neurobiology, Life Sciences, and Interdisciplinary

Center for Neural Computation, Hebrew University, Jerusalem

How do we recall the order of events or items in a sequence? Studying serial memory in monkeys, we found that they prefer to categorize images according to their ordinal position in a list, rather than use item-to-item chaining. Presumably, category assignment is based on an abstract label associated with all images of the same ordinal category (Orlov et al. Nature. 2000; Cerebral Cortex, 2002). We now tested the effect of these presumed ordinal number category labels on priming in human subjects. They were trained with 32 images divided into 8 quadruplets. On each trial they viewed 4 sequentially presented sample stimuli, followed by a test stimulus consisting of the same images. The task was to mouse-click the images in the order of prior presentation. Subsequent memory tests signaled that subjects successfully categorized images according to ordinal position. To test the priming effect, we presented subjects with a prime image - to which they responded by reporting covertly the image category. Then, (ls after prime presentation), they were presented with a pair of target images - to which they reported whether the targets belong to the same category or different categories. We asked whether retrieval of the prime category influences same-different judgment? Performance in trials when prime was of the same category as one or both of the targets was compared with trials with an unrelated prime. Results indicate a significantly decreased reaction time for related primes. This priming effect did not result from a speed-accuracy trade-off and was not influenced by stimulus type (faces or objects). If covert category report was replaced by retrieval of an irrelevant prime feature or passive viewing, categorics are well represented in human cortex and lead to conceptual priming.

Supported by Center of Excellence grant (ISF) and grant from the National Institute for Psychobiology, Israel

In vivo occupancy of female rat brain estrogen receptors by $17 \mathrm{~b}$-estradiol and tamoxifen

Pareto D., Alvarado M., Hanrahan S. and Biegon A.

Dept. Imaging, LBNL, Berkeley CA USA

Estrogens or antiestrogens are currently used by millions of women but the interaction of these hormonal agents with brain estrogen receptors (ER) in vivo has not been fully characterized to date. The goal of the present study was to assess, in vivo, the extent and regional distribution of brain ER occupancy in rats chronically exposed to $17 b$-estradiol $\left(E_{2}\right)$ or tamoxifen (TAM). For that purpose, female ovariectomized Sprague-Dawley rats were implanted with subcutaneous pellets containing either placebo (OVX), $E_{2}$ or TAM for 3 weeks. ER occupancy in grossly dissected regions was weeks. ER occupancy in grossly dissected regions was
quantified with $\left.16 \mathrm{a}-\left[{ }^{18} \mathrm{~F}\right] \mathrm{fluoroestradiol}\left({ }^{18} \mathrm{~F}\right] \mathrm{FES}\right)$ and showed both agents produced significant decreases in radioligand binding in the brain, but the effect of E2 was bigger and more widespread than the effect of tamoxifen. Detailed regional distribution of the interaction was then Detailed regional distribution of the interaction was then
undertaken using an iodinated undertaken using an
$11 \mathrm{~b}-$ Methoxy-16a-125 $]$ iodinated quantitative autoradiography. $E_{2}$ treatment resulted in accumulation throughout the brain while ER occupancy in the TAM group showed a marked regional distribution such that inhibition ranged from $40+1 \quad 15.6 \%$ in the ventromedial hypothalamus to $84.6+1-4.5 \%$ in the cortical amygdala. These results show that exposure to pharmacologically relevant levels of tamoxifen produces a variable, region-specific pattern of brain ER occupancy which may be influenced by the regional proportion of ER receptor subtypes.

\section{Do perceptual learning effects transfer between}

\section{hemispheres and/or between tasks?}

Pavlovskaya $\mathrm{M}^{1}$ and Hochstein $\mathrm{S}^{2}$

'Loewenstein Rehabilitation Hospital. Raanana and Faculty of Medicine, Tel Aviv University; ${ }^{2}$ Life Science Institute, Hebrew University, Jerusalem

Differences in perceptual learning transfer may be related to the cerebral sites of modification due to experience: hard-condition tasks are seen as requiring low-level (specific) representations while easy-condition tasks are performed using high cortical level mechanisms alone (Ahissar \& Hochstein Nature, 1997). We recently found that inter-hemispheric transfer also depends on task difficulty. Subjects practiced a color and orientation feature search task, each within one hemifield. Following training, we switched the sides of the color and orientation tasks. We found nearly complete transfer for easy conditions. and considerably less with difficult conditions. We have discussed the results in terms of inter-hemispheric transfer (Pavlovskaya \& Hochstein. Neural Plasticity, 2002) 
However, an alternative interpretation exists, namely, that the transfer is across task rather than across hemisphere. That is, perhaps training with color pop-out transfers to orientation search in the same hemisphere, and orientation training transfers to color erformance in the other. This result would also indicate a very high cortical level, where the same mechanisms might be responsible for finding odd elements of various features. We tested this alternative by teaching orientation (or color) in one hemifield and a third task (gap presence) in the other and then switching to a new feature for these subjects, color (or orientation) in the fist hemifield, and orientation (or color) in the other. In this case, cross-hemifield transfer would be useful only for the second hemifield. We found considerable cross-task transfer (for easy conditions).

Our results support the notion that feature search with easy conditions is performed at high cortical levels where representation mechanisms include much of the visual field on both sides of the vertical meridian, but also may detect odd elements in a generalized manner.

Supported by Charles E. Smith Laboratory for Collaborative

Research in Psychobiology, Jerusalem; Israel Science

Foundation "Center of Excellence" grant; US-Israel

Binational Science Foundation.

Learning-induced changes in motor cortex

Paz R. ${ }^{1,2}$, Boraud T. ${ }^{1,3}$, Nathan $\mathrm{C}^{\mathrm{l}}$, Bergman $\mathrm{H}^{1,2}$ and Vaadia

${ }^{I}$ Physiology Dep. Hadassah med. sch., Jerusalem, Israel; ${ }^{2}$ The Interdisciplinary center for neural computation, Hebrew

University, Jerusalem, Israel; ${ }^{3}$ Laboratoire de

Neurophysiologie, CNRS UMR 5543, Universite Victor Segalen, Bordeaux, France.

Human studies show that the learning of a new sensorimotor mapping that requires adaptation to directional errors is spatially local. We duplicated these results in monkeys that were required to adapt to visuomotor rotations. Specific changes of activity were only observed in a subpopulation of cells in the motor cortex with directional properties corresponding to the locally learned rotation. Changes adhered to the dynamics of behavior during learning and persisted between learning and relearning of the same rotation. These findings suggest a neural mechanism for the confined generalization of a newly acquired internal model and provide electrophysiological evidence for its retention in motor cortex. We further used information theory to explore learning-induced changes in encoding and decoding. After learning, neurons in the primary motor cortex conveyed more information about the direction of movement and did so with relation to their directional sensitivity. Similar to recent findings in sensory systems, this specific improvement in encoding was correlated with an increase in the slope of the neurons tuning curve. Therefore, our results suggest similar learning mechanisms for sensory and motor areas and provide evidence for a tight relationship between locality of learning and properties of neurons. We further demonstrate that the improved information enables a more accurate reconstruction of movement direction from neuronal populations. This suggests that learning induced changes can be used for better readout of neural activity, both in the brain itself as well as by an external observer.

Tracking-down novel mutations at the regulatory elements of MECP2 gene employing quantitative analysis of relative of MECP2 gene employing quantitative analysis of relatil. Petel-Galil Y. ${ }^{1,3}$ Ben Zeev B. ${ }^{2}$, Goldman B., ${ }^{1,3}$, Gak E., Danek Gertner Genetics Instititute and ${ }^{2}$ Child Neurology Dept., Sheba Medical Center; ${ }^{3}$ Sackler Medical School, Tel Aviv University.

The present awareness that MECP2 provides the possibility of molecular diagnosis of Rett syndrome and other atypical forms, prompts the clinicians to pursue the analysis of MECP2 in an increasing number of cases. However, mutation detection by direct sequencing of the MECP2 coding region, leaves a substantial number of undiagnosed cases in whom the involvement of MECP2 cannot be excluded. We considered an alternative approach based on quantitative MECP2 expression analysis in peripheral blood lymphocytes, intended for identification of novel mutations in the regulatory elements of MECP2. In a preliminary trial we included blood samples of normal subjects, and Rett patients with MECP2 coding and 3 deletion mutations, and a classical patient with no mutation findings. We also obtained samples of lymphoblast cultures generated from patients with known mutations. We monitored MECP2 expression levels using Real-Time PCR and highly specific TagMan probes for (1) MECP2 and (2) $x$-actin and RNaseP reference genes, in order to assess the relative transcript quotient most consistent with the normal and abnormal phenotypes. The MECP $2 /$ RNaseP blood ratio was fouid as a potentially meaningful value that was consistent with mutation type and Rett phenotype. The normal panel was relatively similar according to this parameter, while both 3 , deletion and no coding mutation samples had lower MECP2/RNaseP. In contrast, the coding mutation sample had normal MECP2/RNaseP value. In lymphoblast cultures, all MECP2 mutations had higher MECP2/RNaseP in compare to the normal X expression levels. The MECP $2 / \lambda$-actin ratio was not found consistent in neither blood nor lymphoblast cultures. At present, we pursue to verify these data in a larger panel of patients and controls, and further subject the MECP2 deficient samples to DHPLC analysis of the $5^{\circ}$ and $3^{\prime}$ UTR fragments, in order to identify novel mutations associated with low MECP2 expression.

Correlation between severity of schizophrenia and levels of a 7 acetylcholine receptor $\mathrm{mRNA}$ on peripheral blood lymphocytes

Perl O. ${ }^{1}$, Strous R.D. ${ }^{1,2}$ and Fuchs $S{ }^{1}$

${ }^{I}$ Dept. of Immunology, The Weizmann Institute of Science Rehovot $76100 ;{ }^{2}$ Beer Yaakov Mental Health Center

Nicotinic acetylcholine receptors (AChRs) and in particular the $\alpha 7 \mathrm{AChR}$ have been shown to participate in pathogenesis of schizophrenia. In a recent study we have reported that schizophrenia patients express lower levels of a 7 mRNA in their peripheral blood lymphocytes (PBL), than healthy controls. The decrease in a 7 mRNA levels is not a result of medication management or nicotine consumption. This decrease is in accordance with reports on a deficit in $\alpha 7 \mathrm{AChR}$ in post mortem brains of schizophrenia patients. In the present study we examined whether there is a correlation between the level of a $7 \mathrm{mRNA}$ and severity of schizophrenia. Blood samples were obtained from schizophrenia patients following evaluation verified by two board certified psychiatrists by means of a structured interview according to Structured Clinical Interview for DSM-IV Axis I, Patient Edition guidelines. To provide another control group for analysis among the psychiatrically ill subpopulation, blood samples from patients diagnosed by psychiatric valuation with "severe" personality disorder were also analyzed. Fifty patients with schizophrenia, 15 with personality disorder, and 15 healthy controls were examined. RNA was prepared from isolated lymphocytes and a7 mRNA was determined by RT-PCR followed by quantification of PCR products specific for human a7 AChR by densitometry. As previously reported, a decrease of a7 mRNA levels on PBLs of schizophrenia patients in comparison with controls was observed. Moreover, we found a significant correlation between the CGI score describing the severity of the illness given by a psychiatric evaluation and the level of $\alpha 7$ mRNA in PBL. Personality disorder patients did not show any significant change in a7 mRNA. The correlation demonstrated in this study between severity of schizophrenia and $\alpha 7 \mathrm{mRNA}$ levels in PBL may contribute to identification and evaluation of schizophrenia, and further suggests that a7 AChR may serve as a reliable peripheral marker for the disease

From snail to sciatic nerve: Retrograde injury signaling from axon to soma in lesioned neurons

Perlson E. , Hanz S. ${ }^{1}$, Medzihradsky K. ${ }^{2}$, Darula S. ${ }^{2}$, Munno $\mathrm{D}^{3}$, Massarwa R.', Shaked M. ${ }^{1}$, Gonzalez-Dunia D. ${ }^{4}$, Syed $N{ }^{3}$ 'Burlingame $A$. 2 and Fainzilber $M{ }^{1}$

${ }^{1}$ Dept. of Biological Chemistry, Weizmann Institute, Rehovot, Israel, ${ }^{2}$ Dept. of Pharmaceutical Chemistry, University of California, San Francisco, U.S.A.: ${ }^{3}$ Faculty of Medicine, University of Calgary. Calgary. Alberta. Canada: ${ }^{4}$ Dept. des Retrovirus, Institut Pasteur, Paris, France.

The cell body of a lesioned neuron must receive accurate and timely information on the site and extent of axonal damage, in order to mount an appropriate response. Specific mechanisms must therefore exist to transmit such information along the length of the axon from the lesion site to the cell body. Three distinct types of signals have been postulated to underlie this process, starting with injury-induced discharge of axon potentials, and continuing with two distinct types of retrogradely transported macromolecular signals. The latter include, on the one hand an interruption of the normal supply of retrogradely transported trophic factors from the target; and on the other hand activated proteins emanating from the injury site (Ambron \& Walters, 1996, Molecular Neurobiology 13 : 61-79).

We have used a differential proteomics approach to identify injury-induced retrogradely transported proteins in nerves of the mollusk Lymnaea. Products of a Lymnaea cytoskeletal protein p 51 are highly represented in the screen, and dsRNA knock-down of p51 inhibits regenerative outgrowth of adult Lymnaea neurons. Upon lesion of rat sciatic nerve, we observed the appearance of mp51 (mammalian p51) in the 
axoplasmic compartment, concomitantly with an increase in its interaction with a complex containing dynein. Nerve injury also enhanced an interaction of this protein with signaling molecules. Finally, the regenerative outgrowth of adult DRG neurons in culture was delayed in mp51 null mice. Thus, mp51 binding to a dynein-containg complex provides a scaffold for retrograde trafficking of signaling proteins in injured nerve.

Peripheral acetylcholinesterase facilitation of pro-inflammatory cytokines production in blood and brain Pollak Y ${ }^{1}$, Ben-Menachem O. , Ben-Hur T. ${ }^{2}$, Soreq $\mathrm{H}^{3}$ and Yirmiya $\mathrm{R}^{\text {. }}$

${ }^{\prime}$ Dept. of Psychology, The Hebrew University of Jerusalem; ${ }^{2}$ Dept. of Neurology, Hadassah-Hebrew University Hospital Jerusalem; ${ }^{3}$ Dept. of Biological Chemistry, The Hebrew University of Jerusalem, Israel

Recent findings indicate that peripheral acetylcholine ( $\mathrm{ACh}$ ) inhibits acute inflammation, adding a rapid, localized, and adaptive anti-inflammatory reflex to the known immune and neuroendocrine anti- inflammatory responses. ACh released by the efferent vagus nerve stimulates nicotinic receptors on macrophages. This attenuates the production of pro-inflammatory cytokines(e.g. interleukin-1 $\beta$ (IL-1 $\beta$ ) , TNF- $\alpha$. As inhibitors and antisense suppressors of acetylcohlinesterase (AChE) enhance cholinergic activity, we examined the hypothesis that these compounds would reduce pro-inflammatory cytokines production in the blood and within the brain. Mice were injected intraperitoneally with endotoxin together with tacrine, rivastigmine, neostigmine or EN101, an antisense oligonucleotide targeted at AChE mRNA, and IL-1 $\beta$ and TNF- $\alpha$ levels measured by ELISA $2 \mathrm{hr}$ later. All AChE inhibitors attenuated IL-1 $\beta$ and TNF $\alpha$ production in the blood, as well as IL-1 $\beta$ secretion within the hippocampus. Intriguingly, AChE inhibitors had no effect on IL-1 $\beta$ production within the hippocampus when injected together with endotoxin directly into this brain structure. Furthermore, in mixed glial cultures, exposure to nicotine, muscarine, or $\mathrm{AChE}$ inhibitors in combination with $\mathrm{ACh}$, had no effect on endotoxin-induced $\mathrm{IL}-1 \beta$ production, suggesting that the diminished production of brain IL-1 $\beta \square$ under suppressed AChE activity was due to peripheral rather than central anti-inflammatory effects of $\mathrm{ACh}$ increases. Our findings suggest that circulation $\mathrm{AChE}$ facilitates inflammatory responses in the periphery, which can indirectly promote the production of brain cytokines under inflammatory conditions. These findings point at circulation cholinesterases as new therapeutic targets, the suppression of which may become useful for treating inflammatory-associated diseases

Mechanisms underlying the coordination between the dynamic dimensions of the plasma membrane surface area and the cytoskeleton during neurites retraction

Prager-Khoutorsky $M$. and Spira M.E.

Life Sciences Institute, The Hebrew University of Jerusalem

Two main cellular systems are involved in structural neuroplasticity: the cytoskeleton and the plasma membrane. While the mechanisms of cytoskeleton modulation were extensively studied the roles of membrane budgeting in structural neuroplasticity are less understood. Nevertheless, it is generally assumed that the changes in the membrane surface area adjust to the dimension of the cytoskeleton.

Using cultured Aplysia neurons we examine here the hypothesis that alteration in the balance between constitutive exocytosis and endocytosis play a leading role in neuronal restructuring. Cultured Aplysia neurons totally retract their main axon and neurites, by membrane internalization following brefeldin A (10 $7 \mathrm{~g} / \mathrm{ml})$ application supplement 55). The retraction is the outcome of excess membrane retrieval over exocytosis and is not due to direct effects of BFA on the cytoskeleton. Retraction proceeds by orderly disassembly of the microtubules skeleton. Inhibition of calcium activated proteases by calpeptin $(50 \mu \mathrm{M})$ or the

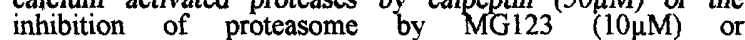
clasto-lactacystin-beta-lactone $(10 \mu \mathrm{M})$ inhibit the process of axon internalization. Imaging of the proteasome proteolytic product accumulation and the calcium concentration revealed that both are elevated in the axon hillock. Since the proteolytic inhibitors do not block membrane retrieval we conclude that BFA-induced retraction is due to blockade of the supply of Golgi derived vesicles on a background of constitutive endocytosis of the plasma membrane and the adjustment of the cytoskeleton dimensions to the reduced surface area. The mechanisms linking between the plasma membrane and the cytoskeleton are calcium-activated proteases localized at initial segment. We conclude that alteration in membrane cycling regulate the cytoskeletal organization and thereby may lead to remodeling of the neuronal cytoarchitecture.

Supported by: The Israel Science Foundation No 556/01
Is speech perception in noise impaired in learning disabled teenagers?

Putter-Katz $\mathrm{H}^{1,2,3}$ and Ahissar M. ${ }^{1,2}$

${ }^{7}$ Dept. of Psychology \& ${ }^{2}$ Center for Neural Computation,

Hebrew University, Jerusalem; 'Speech and Hearing Center.

Sheba Medical Center, Tel Hashomer

Despite the general acknowledgement that noisy environment excessively impairs language comprehension of many individuals with learning impairments, little is known about the underlying processes. Particularly, it is not clear whether impaired function stems from poorer speech perception in noise or from poorer cognitive-linguistic abilities. In the present study we compared the thresholds of speech identification in temporally modulated noise of learning impaired $7^{\text {th }}$ grade students and an age and education matched control group. Subjects were asked to repeat one disyllabic pseudo-word each time. The intensity of the pseudo-words (above the fixed noise level) was adapted in a 3 down/1 up staircase procedure, converging on $80 \%$ correct repetitions. In addition we evaluated the reading, language and working memory performance of the two groups. We found that the learning impaired students performed worse than their peers on the language and memory tasks. However, no difference was observed between the groups in their thresholds for speech identification in noise. Interestingly, in both groups, speech identification thresholds were correlated with memory span for infrequent words, but not with working memory scores. These findings indicate that, in contrast to common assertions, speech perception of learning disabled teenagers is not more sensitive to background noise. Their impaired performance in noisy environments stems either from increased perceptual sensitivity to other parameters (e.g. temporal aspects), or from higher-level cognitive factors.

Synaptic plasticity in the service of homeostasis of neuronal activity in a model cal pyramidal neuron

Rabinowitch I. and Segev I.

Interdisciplinary Center for Neural Computation and Institute for Life Science, Hebrew University in Jerusalem

Extensive experimental work carried out in recent years has demonstrated that neurons employ plasticity as a means for maintaining large time scale homeostasis of their electrical activity. One such form of plasticity is the homeostatic scaling of synaptic conductance, which can successfully counteract overall elevation (or reduction) of membrane potential (e.g. Leslie et al., 2001, J. Neurosci. 21:RC170, 1-6).

Using a detailed compartmental model of a CAl pyramidal neuron, we studied such a mechanism theoretically and found that: (i) local, synaptic-specific homeostatic scaling is capable of equalizing the large time scale average membrane potential throughout the entire dendritic tree, producing a highly non-uniform distribution of synaptic conductance, with dramatic differences between the main apical trunk and the side branches; (ii) a global change in the neuron's presynaptic activation rate results in a global multiplication of synaptic strength, in spite of the local scaling rule being used, consistent with experimental results (e.g. Turrigiano et al., 1998, Nature $391,892-896$ ); (iii) changing the average presynaptic activation rate for just a small segregated subgroup of synapses results in significant rescaling of the strength of these synapses, provided they are clustered on side branches.

We suggest that while phenomena such as voltage fluctuations or LTP link the neuron to the short time scale events taking place in its environment, homeostatic plasticity couples the neuron to substantial and persistent large time scale environmental changes, which determine the significance of those events to the long-lived "life of the neuron"

Reactive microgliosis following organophosphates-induced brain damage: Peripheral benzodiazepine receptors as faithful markers to memory impairment

Raveh L., Brandeis R., Gilat E., Rabinovitz I., Cohen G., Sonego H., and Weissman B.A.

Dept. of Pharmacology, Israel Institute for Biological

Research. Ness Ziona

Regardless of the cause of neuronal injury, reactive glial cells always appear at and around the site of insult. These cells are distinguished by the exceptional abundance of peripheral benzodiazepine receptors (PBRs; w3 sites), particularly as compared to surrounding neurons. Measuring the binding of specific ligands to PBR (e.g., $\left.{ }^{3} \mathrm{H}\right] \mathrm{PK} 11195$ ) offers a unique indirect marker for reliable damage assessment in the CNS. Positron emission tomography (PET) with the PBR ligand ${ }^{11} \mathrm{C} \mid \mathrm{PK} 11195$ is used to monitor human brain neurodegenerative processes (e.g., Alzheimer disease). Numerous animal models demonstrated the existence of a direct relationship between brain neuropathologies and cognitive dysfunction. However, while attempts to relate 
drug-induced neuropathologies to spatial memory impairments in the Morris water maze yielded relatively low correlation coefficients $(r<0.50)$, comparison to the respective PBR densities produced markedly higher values $(r>0.60)$ in rats intoxicated with soman, a potent organophosphate compound. Thus, the estimation of glial proliferation associated with neuronal damage, utilizing simple binding techniques, is shown to be a faithful indicator for the accompanying cognitive deficits.

Assessment of the functional role of the blind's occipital cortex in verbal memory tasks using fMRI

Raz N., Amedi A. and Zohary E.

Hebrew University, Jerusalem, Israel 91904

We recently showed using fMRI, that the occipital cortex of congenitally blind subjects is significantly activated during tasks requiring retrieval of words from memory. These were auditory verb generation (VG) and a verbal memory task, requiring the recall of abstract words lists (VM). No such occipital activation was found in the sighted group. Interestingly, we found that the congenitally blind have superior verbal memory capabilities compared to the sighted. Thus, performance on the standardized Wechsler Memory test (WMS-3 $3^{\text {rd }}$ edition) of $9 / 10$ blind subjects was above the sighted population average. This raises the possibility that the extra activation in the occipital cortex of the blind during performance of verbal-memory tasks, accounts for their superior mnemonic skills. We therefore focused on the correlation between both measures on a subject-by-subject basis. We utilized the fact that both verbal memory capabilities and the degree of occipital activation during VM varied between the blind subjects. Specifically, significant V1 activation ( $p<0.05$ corrected) was found in $6 / 10$ blind subjects. Crucially, strong correlation $(r=0.81)$ was observed between the individual subjects' performance in Wechsler memory test and their V1 BOLD signal intensity during VM. Similar correlation $(\mathrm{r}=0.74)$ was found with long-term memory performance, measured by the percent of words identified as belonging to the original lists used during VM, months after the scan. Using an event-related fMRI, we now test whether the signal in the occipital cortex of the blind is greater for remembered than for forgotten words, as was indicated for typical memory regions. Such an effect will establish the involvement of the occipital cortex in the mnemonic processing of verbal material in the blind.

Supported by Israel Science Foundation grant \#8009.

\section{Socially restricted housing in laboratory rats increases} morphine consumption and increases aggression: Common or separate phenomena?

Raz S. and Berger B.D.

Dept. of Psychology, Brain and Behavior Research Center, University of Haifa, Haifa. 31905

We and others have reported that socially restricted housing increases aggression and interferes with the performance of a cooperation task in male rats. One possible explanation is that social isolation increases or exacerbates irritability or stress both of which are also known to influence drug seeking behavior in humans.

We report here that socially restricted housing significantly increases the drinking of a morphine solution (but not water) in both male and female rats. On the other hand isolation-induced aggression is seen only with males. As in the case of isolation-induced aggression, as little as $60-\mathrm{min}$. of daily physical interaction with another rat completely abolishes the increase in morphine consumption in socially restricted animals.

Daily treatments with the SSRI fluoxetine $(5 \mathrm{mg} / \mathrm{kg}$ i.p.) reverses the increase in morphine consumption consistent with earlier studies that fluprazine $(5 \mathrm{mg} / \mathrm{kg}$ i p blocks the increase in aggression following socially restricted housing. However, whereas we have shown earlier that the beta blocker propranolol $(5 \mathrm{mg} / \mathrm{kg}$ i.p.), reduces isolation-induced aggression, we report here that propranolol does not reduce the morphine consumption increase in animals housed in isolation. In fact propranolol tends to increase morphine consumption in socially restricted animals, while significantly decreasing morphine intake in animals housed socially.

To evaluate the selectivity of these effects, we have begun to evaluate the effects of socially restricled housing on the intake of other substances, in cluding amphetamine, alcohol, and quinine.

From these and other results we consider whether common or separate mechanisms are responsible for the increase in consumption of morphine and the increase in aggression following socially restricted housing. We also discuss whether an increase in irritability or stress following restricted housing best describes the overall results or whether some other explanation(s) may be more appropriate
Neurorescue properties of the green tea polyphenol 3,3-epigallocatechin-3-gallate (EGCG) and its potential

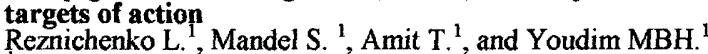
${ }^{I}$ Eve Topf and US NPF Centers for Neurodegenerative diseases and Dept. of Pharmacology, Faculty of Medicine. Technion, Haifa

We have investigated the ability of EGCG to protect and rescue rat pheochromocytoma $\mathrm{PC1} 2$ cells from apoptosis induced by serum withdrawal and the molecular mechanisms involved in these actions. To evaluate neuroprotection, PC12 cells were deprived of serum support and immediately treated with increasing concentrations of EGCG (0.01-1 $\square \mathrm{M}$ ) for 24 and $48 \mathrm{~h}$. Cell viability was significantly reduced $24 \mathrm{~h}$ after serum deprivation, as determined by phase contrast microscopy, showing retracted cell bodies and processes, or as measured by MTT reduction test $(50 \%$ reduction vs control) However, EGCG markedly increased cell survival by $30-60 \%$ over serum free (SF) conditions. The neurorescue effect of EGCG (0.1-10 $\square \mathrm{M})$ was evident when given 24-72h after serum deprivation, partially restoring cell viability to $140 \%$ over SF values, as measured by ELISA. Serum withdrawal caused activation and cleavage of the apoptotic marker caspase-3 (1.67-fold over control), while exposure to 0.1 and 1 $\mu \mathrm{M}$ of EGCG for $24 \mathrm{~h}$ effectively prevented its activation. We also compared the effect of nerve growth factor (NGF), a neurotrophic factor known to induce neurite outgrowth and neuronal survival upon serum deprivation. Similar to EGCG, NGF $(50 \mathrm{ng} / \mathrm{ml}$ ) treatment for 24 or $48 \mathrm{~h}$, prevented and rescued cell damage induced by serum withdrawal. We further examined the levels of phosphorylated (active) and non-phosphorylated serine/threonine protein kinase B (Akt), an established mediator of neuronal survival and axonal elongation. NGF, as expected caused a significant increase in the levels of phospho-Akt in both experimental paradigms (neuroprotection and neurorescue). Similar results were obtained with $0.1-1 \mu \mathrm{M}$ EGCG increasing the levels of phospho-Akt, compared to untreated control. This study shows for the first time that EGCG displays not only neuroprotective action, but also neurorescue properties, involving long-term activation of the PI3/Akt pathways, as well as inhibition of apoptotic pathway.

BOLD-fMRI of the rat barrel field cortex in response to

multiple whisker stimulation

${ }^{7}$ MRI/MRS lab, HBRC, Medical biophysics and nuclear medicine dept., Hadassah - Hebrew University Hospital

In the somato-sensory cortex in the rat brain, each whisker from the snout is represented as a highly localized region in layer 4 , which are termed 'barrels'. When the neurons in the barrel are excited by whisker movement the excitation stays localized specifically to the area in the barrel whereas in layer $2 / 3$ the area directly above the barrel receives the excitatory input which can propagate laterally as has been previously documented from electro-physiological studies (1). Additional studies have shown that there is a correlation of the neural spike count amplitude in response to different whisking frequencies (2)

In this study we examined the BOLD-fMRI response in the rat barrel field cortex as a result of multiple whisker deflection. We aim to further explore the MRI signal in response to whisker deflection at a variety of frequencies in order to compare to the results obtained from electro-physiological experiments and to better understand the source of the BOLD signal.

Methods: The rats were anaesthetized with ketamine and secured in a homemade holder similar to a stereotaxic frame. A surface coil of $15 \mathrm{~mm}$ was placed directly over the barrel field cortex. The whiskers were stimulated by air puffs directed at the central whiskers at a frequency of $5 \mathrm{~Hz}$. A fast imaging protocol was used. EPI with a TE of $17.96 \mathrm{msec}$ and a spatial resolution of $156^{*} 156 \mathrm{~m}$. The whisker stimulation paradigm consisted of alternating ON and OFF periods where each period lasted a course of one minute.

Results: The data obtained was analyzed by using a box-car correlation test. Data sets showing only activation in the superficial layers of the cortex were excluded. We obtained 4 images with activation in the contralateral barrel cortex which was not restricted solely to layer 4 , there was also a much less significant activation in the ipsilateral barrel cortex

1 Petersen CCH et al.(2003) J Neurosci 23:1298-1309; 2 Ahissar E et al. (2001) J Neurophysiol 86:354-367 
Conformational rearrangements associated with the gating of the $G$ protein coupled potassium channel revealed by FRET microscopy

Riven I., Kalmanzon E., Segev L. and Reuveny E

Dept. of Biological Chemistry, Weizmann Institute of Science, Rehovot 76100

$\mathrm{G}$ protein coupled potassium channels (GIRK/Kir3.x) are key determinants that translate inhibitory chemical neurotransmission into changes in cellular excitability. These channels are activated by the binding of the GDD subunits of $G$ protein in concert with other intracellular components. To understand the mechanism of channel activation by $G$ proteins it is necessary to define the structural rearrangements in the channel that result from interaction with G[J I subunits. In this study we used a combination of fluorescence spectroscopy and through-the-objective total internal reflection microscopy to monitor the conformational rearrangements associated with the activation of GIRK channels. Conformational changes were assessed from changes in the efficiency of fluorescence resonance energy transfer (FRET) between CFP and YFP attached at various positions in the cytosolic domains. We detect activation-induced changes in FRET consistent with a rotation of the C-termini along the central axis of the channel. We propose that rotation and expansion of the $C$-termini drives the channel to open by bending and possibly rotating the second transmembrane segment.

Neuronal repair by disaccharides derived from chondroitin

Rolls A. ${ }_{2}$, Avidan $\mathrm{H}^{2}{ }^{2}$ Cahalon $\mathrm{L}^{2}$, Schori $\mathrm{H}^{1}$, Bakalash S. , Lider O. ${ }^{2}$, Schwartz ${ }^{\prime}{ }^{1}$

Depts. of Neurobiology and Immunology $y^{2}$, The Weizmann Institute of Science, Rehovot

Chondroitin sulfate proteoglycans (CSPGs) inhibit central nervous system (CNS) axonal regeneration, and their local degradation promotes CNS recovery. We suggest that CSPGs act as nesting molecules in CNS repair; intact CSPG demarcates the site of injury, supports the immune reaction mediated by $T$ cells and promote the activation of microglia to a neuroprotective phenotype. Upon degradation CSPG provides disaccharides (DSs) whose function is the repair of damaged neurons. We have shown that a CSPG-derived DS, via activation of PKC and Pyk2 in neurons, induced neuronal survival and neurite outgrowth prevented lysophosphatidic acid-induced collapse of neurites, and protected cultured hippocampal neurons from death. Moreover, the CSPG-DS further supports the microglial activation to sustain its neuroprotective phenotype. Systemically injected disaccharides protected neurons from degeneration in rodent models of acute glutamate intoxication and chronic glaucoma. CSPG-DS might provide means of circumventing common extracellular toxic or growth inhibitory compounds, characterizing the neurodegenerating circumstances. Exogenous supply of CSPG-DS might be a way to promote repair after acute CNS injuries or in chronic neurodegencrative conditions.

Venom reveals descending control of a modulatory system which regulates the expression of locomotory behaviors Rosenberg L.A and Libersat $F$.

Zlotowski Center for Neuroscience and Dept. of Life Sciences, Ben-Gurion University of the Negev, Beer-Sheva

In insects, locomotion is generated in the thoracic central nervous system. The wasp Ampulex compressa injects a neurotoxins cocktail into the brain of its prey to induce a long lasting change in the initiation and execution of locomotory behaviors. For instance, the thoracic escape running neuronal circuit of a stung animal is impaired at the level of central synapses between thoracic interneurons to motoneurons. These central synapses are known to be modulated by bioamines. Our hypothesis is that the injected venom affects neurons located in the brain, which send descending tonic input to bioaminergic neurons. These, in turn, control the thoracic premotor circuitry. Octopamine is a bioamine, which is secreted by DUM (dorsal unpaired median) neurons in the prey's thoracic portion of the nervous system. It is known to regulate the expression of specific motor patterns by modulating the excitability of specific neurons.

The purpose of the present investigation was to examine the descending control exerted on the DUM neurons. This was approached by recording the activity of DUM neurons either after removing the brain or after an injection of the venom in the brain. We show that the activity of an identified DUM neuron from the metathoracic ganglia is altered in stung and brainless animals. The spontaneous firing rate of this DUM neuron in stung and brainless animals is 3 and 6 folds lower then control animals, respectively.
Given these results, we propose that the venom injected into the brain affects descending tonic input to the thoracic DUM neurons in a more subtle way then abolishing all descending input from the brain. The decrease in activity of the DUM neurons could be responsible for the decrease in the responsiveness of thoracic motor circuits and consequently the expression of specific locomotory patterns.

\section{Naturally occurring anti-tau antibodies in the sera of patients affected by Alzheimer's disease and in healthy} aged subjects

Rosenmann H., Steinitz M., Meiner M., Geylis V. and

Abramsky $\mathrm{O}$

Dept. of Neurology, Hadassah University Hospital, Jerusalem, and Experimental Pathology, The Hebrew

University-Hadassah Medical School, Jerusalem

Objective: To investigate whether naturally occurring anti-tau antibodies (Abs) are present in the sera of patients affected by Alzheimer's disease (AD), and whether the titers of these Abs differ between $A D$ patients and healthy subjects. The finding may point to a role that these Abs might play in the pathogenesis of the disease.

Background: A possible role of autoimmunity in the pathogenesis of $\mathrm{AD}$ has lately attracted a great attention Immunization against beta-amyloid has been reported to reduce amyloid burden and attenuate $A D$ pathogenesis, but some neuroinflammatory complications were reported Moreover, naturally occurring anti-beta-amyloid Abs have been detected in sera of $\mathrm{AD}$ patients and also in healthy adults and aged individuals, but their role is not fully understood. As for the second main component involved in the pathogenesis of $A D$ - the tau protein, it is unclear whether naturally occurring Abs targeted against the tau protein are present in the sera of $A D$ patients, and whether they are involved in disease pathogenesis

Methods: We developed a sensitive anti-tau ELISA using the full length recombinant human tau protein as antigen. The assay was used to analyze the titers of anti-tau Abs in sera of $A D$ patients and age matched controls.

Results: Naturally occurring Abs directed against tau were detected in the sera of AD patients and in healthy age matched controls. The titers of the Abs in the AD patients and in the controls were comparable, with no significant difference.

Conclusion: In this study we demonstrated the presence of autoantibodies in the sera of AD patients, and also in healthy aged individuals, with comparable titers. A larger sample is needed to confirm these results. We intend to compare not only the titers but also the isotypes of anti-tau Abs in AD patients and in healthy controls. Today, it is unknown if these anti-tau Abs are neuroprotecive or rather neurotoxic, similarly to the uncertainty with regards to the role of anti-amyloid- beta Abs in the pathogenesis of $\mathrm{AD}$.

Effects of sensory deprivation on barrel synapses in the adult mouse

Sadaka Y., Weinfeld E. and White E.L.

Morphology, Ben Gurion University of the Negev, Beer Sheva

Deprivation induced changes in the functional organization of the receptive fields of barrel neurons occur in animals deprived from birth as well as in those deprived as adults. In a previous study, trimming whiskers from birth through $\mathrm{P} 60$ caused decreases of $52 \%$ and $43 \%$ respectively, in the numerical densities of symmetrical and thalamocortical synapses in layer IV (Sadaka et al., 2003).

In this study the effects sensory deprivation in adults is examined. Thalamocortical synapses were labeled by lesion-induced degeneration in adult mice at $\mathrm{P} 60$ and $\mathrm{P} 100$ subjected to whisker deprivation from $P 40$. After fixation, cerebral hemispheres contralateral to the deprived side were sectioned at $50 \mu \mathrm{m}$ and embedded in plastic for thin sectioning. An unbiased stercological approach was applied blindly to systematic random samples of neuropil in barrel hollows to estimate the numerical density of al asymmetrical synapses, thalamocortical and symmetrical synapses, and compared in trimmed vs. control animals. Preliminary results indicate no differences in the numerical densities for any class of synapses between deprived and control animals. Thus, the considerable plasticity of the thalamocortical and symmetrical synapse populations consequent to deprivation beginning at birth is not reproduced when deprivation begins at $\mathrm{P} 40$. Supported by the Israel Science Foundation grant no. 52/00 to ELW 
AD4, a novel brain target antioxidant attenuates haloperidol-induced dyskinesia in rats Sadan O. ${ }^{1}$, Stroomza M. ${ }^{1}$, Gilgun-Sherki Y. ${ }^{1}$, Atlas D. ${ }^{2}$,

Dept. of Neurology and Felsenstein Medical Research Center Rabin Medical Center, Sackler School of Medicine, Tel Aviv University, Petah Tikva; ${ }^{2}$ Institute of Iife Sciences, Hebrew University, Edmond Safra Campus, Jerusalem

The acute and chronic treatment for schizophrenia and other psychotic disorders with anti-psychotic drugs has several side effects. tardive dyskinesia (TD) is a late onset chronic side effect, in about $30 \%$ of those patients which is characterized by abnormal movements. Oxidative stress and release of free radicals molecules attaching to dopaminergic system is one of the leading theories explaining TD's pathopysiology. In light of this assumption, we chose to try a novel low molecular weight thiol antioxidant (AD4) as a possible treatment for TD. In order to examine the characteristics of TD we used the anti-psychotic drug, haloperidol, to include experimental model in rats. The typical vacuous chewing movement (VCM, ) was occurred in rats following six weeks of chronic injection. This purposeless mouth opening in the vertical plane is similar to the human TD symptoms. We found that haloperidol increased VCM level from $16.4 \pm 2.4$ in untreated rats to $66.5 \pm 7.6(\mathrm{P}<0.01)$, while co-administration of haloperidol and AD4 decreased VCM level to $42.1 \pm 6.7(\mathrm{P}<0.05)$. Haloperidol also increased the level of lipid peroxidation and protein oxidation in the rat's brain, as was measured by the TBARS and carbonyls methods, while co-administration with AD4 preserved their normal levels. In conclusion, haloperidol causes behavioral abnormalities similar to TD in rats associated with oxidative stress. AD4 the brain-targeted potent antioxidant, reduces the cellular oxidation markers and improves the typical clinical behavior. Hence, AD4 is a potentially new treatment for anti-psychotics-induced TD.

\section{Expression of ShcC proteins in mouse brain: Effect of age} and LPS

Sagi O., Wolfson $\mathrm{M}$. and Fraifeld V

Dept. of Microbiology and Immunology, CMRA, Ben-Gurion University of the Negev, Beer-Sheva

The family of recently discovered She proteins includes ShcA ShcB, and ShcC. They act as adaptors coupling activated receptors with tyrosine kinase activity to downstream signaling molecules, including the MAPK-associated pathway. Expression of ShcB and ShcC appears to be limited to neuronal tissue. Lipopolysaccharide (LPS), a component of the cell wall of gram-negative bacteria, is a classic trigger of inflammatory reactions accompanied by a generation of reactive oxvgen species and activation of MAPKs. The brain is one of the LPS targets and the response to LPS is altered with age. The present study was aimed at examining the expression of ShcC in the mouse brain with respect to animal's age and the effect of LPS. Male CD-1 mice aged 4 days to 12 months old were injected intraperitoneally with $1 \mathrm{mg} / \mathrm{kg}$ LPS and at different times post-injection, their brains were removed for preparation of tissue extracts. The latter were processed through Western blot analysis using specific anti-ShcC antibodies. We found that (i) the basal level of ShcC in mouse brain increased gradually during the postnatal period, reaching the maximum at 2 months. Middle-age mice had a lower level of ShcC compared to the young adult and young animals; (ii) both p55 kDa and p $69 \mathrm{kDa}$ isoforms of ShcC displayed similar age-related patterns; (iii) LPS differentially affects the expression of ShcC isoforms, resulting in a marked up-regulation of $\mathrm{p} 69$ and an insignificant effect on the level of p55; (iiii) the effect of LPS on ShcC was age-related, being more pronounced in the younger mice compared to the older animals.

Supported by a grant from the United States-Israel Binational Science Foundation (BSF). Jerusalem, Israel.

Correlation between firing rate and spontaneous synaptic activity in SCN neurons

Sagiv N. and Yarom Y.

Dept. of Neurobiology, Life Sciences, Hebrew University Jerusalem

Circadian behavior in mammals is governed by the hypothalamic suprachiasmatic nucleus (SCN). GABA (7-amino butyric acid) is an abundant neurotransmitter in the $S C N$. Most SCN neurons contain GABA and show GABAergic synaptic activity, mediated mainly by GABA receptors. Yet, it is not clear to what extent SCN neurons form an intrinsic GABAergic network. In order to unravel the network connectivity in the circadian clock we recorded synaptic and firing activity of SCN neurons. Recordings were made from a $3005 \mathrm{~m}$ hypothalamic slice containing the SCN Both the firing rate and the frequency of spontaneous synaptic currents were found to be high during the day $(4.69 \pm 1.96$ and $27.45 \pm 20.10 \mathrm{~Hz}$, respectively). A low rate of activity was found during the night $(3.27 \pm 3.10$ and $17.68 \pm 18.46 \mathrm{~Hz}$ for firing rate and synaptic currents, respectively). The rate of TTX insensitive spontancous synaptic currents was $2.10 \mathrm{~Hz}$ independent of the circadian time. The calculated ratio between firing and synaptic activities was 5.8 during the subjective day and 5.4 during subjective night. Hence we concluded that a) on average there are 5 GABAergic synapses on each SCN neuron and b) the efficiency of the synaptic release mechanism seems to be independent of the circadian time. These results strongly support the hypothesis that the $\mathrm{SCN}$ is composed of a network of interconnected GABAergic neurons.

Genomic and proteomic profiling of the neuroprotective mechanisms of rasagiline in the mouse MPTP model of PD Sagi Y. ${ }^{1}$, Mandel S. ${ }^{1}$ and Youdim MBH.

${ }^{T}$ Eve Top and NPF Cntrs of Excellence for Neurodegenerative Diseases Research, and Dept. of Pharmacology. Technion Faculty of Medicine, Haifa

Rasagiline is a monoamine oxidase (MAO) $\mathrm{B}$ inhibitor antiParkinson's disease (PD) drug Its neuroprotective properties has been examined in many cell culture as well as froperties has been examined in many cell culture as well as and monkeys. These studies and cDNA microarray gene expression have indicated involvement of $\mathrm{Bcl}-2$ family proteins and other anti apoptotic genes. In order to reveal these mechanisms we adopted two novel methods: genomic and proteomic profiling systems, in the mouse model of MPTP. Both RNA as well as protein fraction were produced from midbrains of mice, cDNA was synthesized and was hybridized to microarray gene chip (Clontech, CA, USA), containing $\sim 2000$ genes and were analyzed using a software (Atlasimage Clontech, USA), while protein samples were detected and analyzed in Powerblot Proteomic array system (BD, CA, USA) containing over $1000 \mathrm{mAbs}$. Gene analysis confirmed that MPTP treatment involves proapoptotic mechanism and proteomics suggested that the INK pathway is recruited by MPTP, in addition to Fas ligand involvement. Furthermore, MPTP involved altered expression of n-nitric oxide synthase (nNOS) glutamate receptor and phosphorylated form of Glycogen Synthase Kinase-3b (GSK3). Proteomic analysis confirmed many of the gene expression profile related to MPTP neurotoxicity On the contrary, rasagiline reversed the pro apoptotic action of MPTP, enhanced anti apoptotic, growth factor and in particular $\beta-\mathrm{NGF}$, its downstream transcription factors: EGR1 (NGF-1 A binding protein) and EGR3 (early growth response protein-3) gene expressions. Rasagiline's ability to upregulate the protein levels of pro-survival signaling of Ras, PI3K, and AKT indicates that part of its neuroprotective mechanism of action is mediated via $\beta$-NGF receptor, TRK-A

Experimentally based detailed model of synaptic connections between layer 4 spiny neuron onto layer $2 / 3$ pyramids in the barrel cortex

Sarid L. ', Sakmann B. ${ }^{2}$, and Segev I. ${ }^{13}$

Tnstitute for Life Science, Hebrew University Jerusalem

${ }^{3}$ Interdisciplinary Cntr for Neural Computations, \& ${ }^{2}$ Dept. of Cell Physiology, Max-Planck Institute for Medical Research Heidelberg

In rodents, whisker movement is somatotopically represented in the "barrel" cortex by electrical activity in the corresponding cortical columns. This orderly mapping is extremely helpful when trying to understand how sensory information is processed within and between cortical columns. The present study is a first step in an endeavor to construct an in silico model of the "barrel" cortex that is tightly based on detailed morphological and physiological information from in vivo and in vitro studies. The excitatory connection between layer 4 spiny stellate cells and layer $2 / 3$ pyramids was modeled here, based on morphology and physiology of layer $2 / 3$ neurons. together with the dendritic location and properties of layer 4 to layer $2 / 3$ synaptic connection obtained from in vitro pair-recordings. Results from in vivo whole cell patch recordings following whisker stimulation, both from layer 4 and layer $2 / 3$ neurons, were used as a target for the model performance. The compartmental models for three layer $2 / 3$ neurons predict an $R_{m}$ around $15 \mathrm{KWcm}{ }^{2}$ and $C_{m}$ around $0.9 \mathrm{mF} / \mathrm{cm}^{2}$ with $R_{i}=150 \mathrm{Wcm}$ and a peak synaptic conductance change, $g_{p e a k}$, that ranges between $0.15 \mathrm{nS}-1.7 \mathrm{nS}$ (corresponding to $40-500 \mathrm{pA}$ peak synaptic current per connection). An error that ranges between $50 \%-80 \%$ in experimental estimation of $g_{p e a h}$ is expected due to imperfect space-clamp. Next, population of 350 layer 4 neurons converging onto a single layer $2 / 3$ were activated at probability and times as measured experimentally following whisker stimulation. This input gave rise to a composite modeled EPSP that ranges between $10-50 \mathrm{mV}$ with a significant NMDA contribution. Suggestions for follow-up experiments are provided together with suggestions for the next level of modeling. 
Signal attenuation leads to excessive pressing in normal humans similar to that seen in rats, and this behavior correlates with the checking and doubting subscales of the Obsessive Compulsive Inventory

Schönberg T and Joel D.

Dept. of Psychology, Tel Aviv University, Tel Aviv

Obsessive compulsive disorder (OCD) is a psychiatric affliction with a lifetime prevalence of $1-3 \%$. We (Joel \& Avisar 2001, Behav Brain Res 123:77-87) have recently developed a new animal model of OCD, the signal attenuation model. This model is based on the assumption that obsessions and compulsions result from a failure to cease responding after the successful completion of an action, due to a deficient signaling that the conditions have changed following the organism's response. In the model, excessive lever-pressing unaccompanied by an attempt to collect a reward is induced by attenuating a signal indicating that the lever-press response was effective in producing food. This 'compulsive' lever-pressing in rats has a pharmacological profile similar to obsessions and compulsions in OCD patients, and depends on similar neural substrates.

The present study tested whether signal attenuation induces a similar behavioral pattern in normal human subjects. Twenty two $1^{\text {st }}$ year social science B.A students underwent a computer task, resembling a simplified version of pinball, which was analogous to the rats' post-training signal attenuation procedure. Twenty other subjects underwent the same task, but without the signal attenuation stage. Subjects undergoing signal attenuation tended to exhibit a higher proportion of 'compulsive' mouse-clicking (calculated as the number of mouse-clicks not followed by an attempt to obtain a reward divided by the total number of mouse-clicks) compared to subjects that have not undergone signal attenuation. Moreover, 'compulsive' mouse-clicking was significantly correlated with the Checking and Doubling subscales of the Obsessive Compulsive Inventory only in the signal attenuation group. This is the first demonstration that the post-training signal attenuation procedure can be conducted in humans. Moreover the present results suggest that the human analogue of 'compulsive' lever-pressing may be relevant to checking symptoms in OCD.

\section{Early acoustic experience is necessary for sound} localization mechanisms in mammals

Seidl A.H. and Grothe B.

Max-Planck-Institute of Neurobiology, Am Klopferspitz 18 a 82152 Martinsried, Germany

Interaural time differences (ITD) occurring at the two ears are a major cue for sound localization. In mammals, ITDs are encoded in the medial superior olive (MSO) by a complex temporal interaction of binaural excitatory and inhibitory inputs. Glycinergic inhibition from the medial nucleus of the trapezoid body (MNTB) seems to be important for tuning ITD sensitivity to the physiological relevant range of ITDs (Brand et al., Nature 417:543, 2002). In normally developing animals this glycinergic input undergoes a specific structural refinement during the first days after hearing onset. This refinement depends on auditory experience and fails to develop in animals, which are reared in omnidirectional white noise designed to mask most spatial acoustic cues (Kapfer et al., Nat. Neurosci $5: 247,2002$ ).

Here we show, for the first time in mammals, that early experience during a critical period is involved in adjusting the ITD-sensitivity of single neurons in the mammalian auditory brainstem. IT $n$ tuning in animals at postnatal day $P 15,3$ days after hearing onset, (P12) significantly differs from that found in adult control animals. Animals that have been raised in an environment with omnidirectional noise from P10 to P25 fail to develop ITD-tuning as found in adult controls. In juvenile and noise-reared animals the ITD-tuning is not consistently adjusted to the range of naturally occurring ITDs. Interestingly ITD sensitivity of juvenile and noise-reared animals is similar to that of MSO neurons during blockade of glycine. In adult gerbils the same omnidirectional white noise did not alter ITD-tuning permanently

This study shows that experience of spatial acoustic cues is fundamental for developing correct low frequency sound localization capabilities. Furthermore, it seems likely that the structural effects of rearing animals in omnidirectional noise are correlated with the functional differences in ITD tuning. The development of inhibitory inputs and the resulting maturation of sound localization mechanisms due to spatia acoustic experience represents a mechanism of direct adjustment of neuronal processing to behaviorally relevan cues and offers an example of activity-driven, self-organized synaptic plasticity of inhibitory inputs.
Epileptiform activity in the blood-brain barrier disrupted cerebral cortex

Seiffert E. ${ }^{1}$, Dreier J.P. ${ }^{2}$ Heinemann W. ${ }^{1}$ and Friedman A ${ }^{1,3}$ "Institute of Physiology ${ }^{2}$ Exp. Neurology, Charit, Humbold University, Berlin, Germany; ${ }^{3}$ Zlotowski Center of

Neuroscience, Ben Gurion University, Beer Sheva, Israel

Epilepsy is one of the most common neurological diseases affecting $1-2 \%$ of the population. The electrical activity characterizing cortical neurons involved in epileptic activity is well documented in different models of the disease as paroxysmal and hypersynchronous activity of large numbers of neurons. However, the mechanisms and sequence of events leading to this abnormal activity are mostly unknown. Here we report a new animal model for direct, focal disruption of the blood-brain barrier (BBB) in the rat somatosensory cortex using low concentrations ( $<5 \mathrm{mM}$ ) of the sodium salts dehydrocholic acid (DHC) or 7-deoxycholic acid (DXC). Electrophysiological recordings 4-14 days following treatment revealed a focus of paroxysmal inter-ictal like epileptiform activity limited to the treated region. Paroxysmal discharges were associated with enhanced glutamatergic excitation, but prominent inhibitory transmission was also found in the BBB-treated region. Focal cortical superfusion with electrolytic solution in serum concentrations did not cause cortical dysfunction, whereas focal application of rat serum, denaturated serum and an electrolytic solution containing albumin resulted in focal paroxysmal activity, suggesting increased oncotic pressure to be sufficient to initiate the chain of events leading to network reorganization and epileptiform activity. While compromised BBB has been described under numerous neurological conditions, it is the first time experimental evidence is given for a specific brain pathology resulting from $\mathrm{BBB}$ breakdown. Transmitter release under high transmitter concentration

Dept. of Neurobiology, Hebrew University, Jerusalem 91904 ${ }^{2}$ Dept. of Computer Science and Applied Mathematics.

Weizmann Institute, Rehovot 76100

50 years ago it was established that $\mathrm{Ca}^{2+}$ is essential for promoting the physiological depolarization-induced phasic transmitter release. Astonishingly, papers attempting to re-confirm this role of $\mathrm{Ca}^{2+}$ still occupy an impressive volume of the published literature. In the last two decades, evidence has accumulated showing that under physiological conditions membrane potential, per se, plays a pivotal role, in addition to $\mathrm{Ca}^{2+}$, in promoting transmitter release, and the Ca-voltage hypothesis, $\mathrm{CVH}$, was suggested. The postulated controlling role of membrane potential has recently been challenged by findings suggesting that depolarization, as such, does not play a role in the physiological release (1). We show here that the lack of effect of depolarization on release is a direct consequence of the unphysiological experimental protocol cmployed in (1). The protocol included long $\mathrm{Ca}^{2+}$ pulses, long depolarization and uncaging of $\mathrm{Ca}^{2+}$ prior to depolarization. Thus, before the depolarization the transmitter concentration in the cleft rises significantly due to release induced by $\mathrm{Ca}^{2+}$ uncaging. Consequently, a fast autoinhibition process, which differs from the slow second-messenger mediated feedback inhibition, inhibits depolarization-induced release. This inhibition occurs mainly during, and shortly after the (long) depolarization pulse. Crucially, the fast autoinhibition is a direct consequence of the molecular model that describes the physiological depolarization-induced release. Accordingly, the ack of effect of depolarization on release in the experiments of (1) can be fully accounted for by the CVH. Hence, not only do these experiments not contradict the $\mathrm{CVH}$, but, rather, they provide further support to the assertion that under physiological conditions membrane potential, as such, plays an essential role in promoting selease.

Protective autoimmunity: Interferon-g activates microglia to scavenge glutamate without evoking oxidative and inflammatory mediators

Shaked $1 .{ }^{1}$, Tochros D. , Gersner $\mathrm{R}^{1}$, Mordechay $\mathrm{S}^{1{ }^{*}}$, Xiao $\mathrm{X}^{2}$ Soteropoulos $\mathrm{P}^{3}$, Tolias $\mathrm{P}^{3}$ Hart R.P ${ }^{4}$ and Schwartz $M$ ${ }^{1}$ Neurobiology, Weizmann Ins., Rehovot. Israel: ${ }^{2}$ Program in Computational Biology, Rutgers U., Newark, NJ. ${ }^{3}$ Center for Applied Genomics. International Center for Public Health,

Newark $N J \cdot{ }^{4} W M$ Keck Center for Collaborative

Neuroscience, Rutgers U. Piscataway, N.J

Glutamate at above-normal concentrations is a common player in neuronal degeneration. The adaptive immune system, via homing of $T$ cells to their specific self-antigens at the site of a central nervous system insult and their local activation there, can help protect against glutamate toxicity. Here we show that activated helper $T$ (Th1) cells increased the ability of microglia to scavenge glutamate. The Thl cytokine interferon (IFN)- $\gamma$ 
simulated this effect, without inducing an oxidative imbalance caused for example by expression of the inducible nitric oxide synthase (iNOS) and without activating inflammation-mediating enzymes such as cyclooxygenase-2 (COX-2), known to impair glutamate uptake. Analysis of TLansducer and activator protein), and IKB (inhibitor of the transcription factor NF-kB) revealed that the adaptive immunity (activated T cells via IFN- $\gamma$ ) activate micoglia via a non-innate pathway, known to be induced by non-innate pathway, known to be induced by neuron-threatening environment, to eliminate destructive self-compounds without hazardous products of the NF-KB pathway. Gene array analysis of IFN- $\gamma$-activated microglia disclosed up-regulation of gene clusters associated with defense against oxidative stress. Our results suggest that microglia, when activated by Thl cells, display a significantly increased ability not only to perform their usual immune-related functions, but also to scavenge potentially neurotoxic glutamate without risk of redox imbalance, thus serving as stand-by neural cells in situations of stress.

An inositol depletion mechanism for valproate Shaltiel G. ${ }^{1}$, Shamir A. ${ }^{1}$, Shapiro J. ${ }^{1}$, Ding D. ${ }^{2}$, Dalton E ${ }^{3}$ Bialer M. ${ }^{4}$, Harwood A.J. ${ }^{3}$, Belmaker RH ${ }^{4}$, Greenberg M.L.' and Agam G.

${ }^{\prime}$ Zlotowski Center for Neuroscience, Ben Gurion University of the Negev, Beersheva, Israel; ${ }^{2}$ Wayne State University, Detroit, Michigan USA. ' University College London, London WCIE 6BT, UK.; ${ }^{4}$ The Hebrew University of Jerusalem, Israel Lithium salts (lithium) and valproic acid (VPA $)$ are the FDA-approved drugs for treating the mood disorder manis deprescive illness (hipnlar disordes). The mechanism of mood-stabilization has not been elucidated, but the role of inositol metabolism has gained substantial support. Lithium inhibition of inositol monophosphatase (IMPase), an enzyme required for inositol recycling and de-novo synthesis suggested the hypothesis that lithium depletes brain inositol and attenuates phosphoinositide-signalling. Previous results indicate that VPA also depletes inositol in yeast, Dictyostelium and rat neurons, but no molecular mechanism was demonstrated. In the present study we show a $20 \%$ reduction of inositol in mouse frontal cortex following acute VPA administration Assays of crude homogenate of human prefrontal cortex indicate that inositol reduction can be explained by a decrease in myo-inositol-1-phosphate (MIP) synthase activity; $0.21 \mathrm{mM}$ VPA, a therapeutically-relevant VPA concentration reduces the activity by $50 \%$. Among psychotropic drugs, the effect is specific to VPA and is not obtained with other anticonvulsant mood stabilizers, typical and atypical antipsychotics, or tricyclic antidepressants. Accordingly, only VPA upregulates expression of the yeast INOI gene coding for MIP-synthase. The VPA derivative N-methyl-2,2,3,3,-tetramethyl-cyclopropane carboxamide (M-TMCD) reduces crude human brain homogenate MIP synthase activity and has an effect similar to VPA on rat neurons, whereas another VPA derivative, valpromide (VPD), poorly affects the activity and has no effect on neurons. These results suggest that the rate-limiting step of inositol biosynthesis, catalyzed by MIP synthase, is affected by VPA, and demonstrate that inositol depletion is a first event common to both lithium and VPA.

The involvement of the asymmetry of the frontal-striatal circuitry in social cognition in patients with Parkinson's disease and in patients with prefrontal brain damage Shamay-T soory S.G. ${ }^{1,2}$, Aharon-Peretz J. ${ }^{\text {, , Goldsher Y. D. , }}$ ${ }^{\prime}$ Cognitive Neurology Unit, Rambam Medical Center, Haifa, ${ }^{2}$ Dept. of Psychology, University of Haifo

The role of the right hemisphere and the prefrontal cortex in social cognition has been well documented in the literature. The purpose of the present study was to examine the contribution of the asymmetry of the fronto-striatal circuitry to social cognition, in view of recent findings, suggesting that the basal ganglia may play an important role in emotion processing and thus contribute to social cognition.

The level of basic and complex social cognition was assessed in patients with localized unilatelal prefrontal $(\mathrm{N}=26)$ and posterior $(\mathrm{N}=17)$ cortical damage, patients with unilateral onset of idiopathic Parkinson's disease ( $P D, N=21$ ) and healthy controls $(N=19)$. Basic aspects of social cognition were assessed using tests of affect recognition through facial expression and through prosody. Complex aspects of social cognition were assessed using empathy scales and theory of mind (TOM) tasks. Results indicated that both PD patients whose motor signs began on the left side of the body (right basal ganglia dopamine deficit), and patients with right prefrontal cortical damage showed impaired recognition of emotions as compared to healthy controls. However, only patients with prefrontal damage showed deficits in the empathy and the TOM tasks, indicating impaired complex social cognition abilities.

The results of the present study indicate an involvement of both cortical and subcortical structures of the right hemisphere in processing of emotional stimuli. It appears that the righ frontal-striatal circuitry is involved in basic affective processing whereas the frontal lobes have a more integrative role in mediating more complex processing of affect.

\section{Spatial cueing does not affect spatial summation Shani R. and Sagi D.}

Weizmann Institute of Science, Rehovo

Attention has been suggested to affect lateral interactions between low-level visual filters (Freeman et al , 2001). In light of this result, we asked how attention would affect spatial summation of a stimulus that is assumed to consist of excitatory lateral interactions such as in an array of low-contrast, co-oriented Gabor patches. In order to do so, we compared the psychometric curves of sixteen conditions in a spatial cueing paradigm. There were two configurations, uniform (in which all Gabor patches had the same orientation) and random; two array sizes- 2X2 (three locations- near fixation midway and far) and $5 \times 5$; and two attentional statescued and un-cued. The target appeared in one of the four visual inid juadrants that were each marked by a white circle in the un-cuea condition. In the cued condition, the circle of the cued quadrant was black. There were two main outcomes: 1) Thresholds in the uniform condition were lower than those in the Random condition. This suggests a cooperative activity between co-oriented stimuli, and strengthens the notion of excitatory lateral interactions between low-contrast co-aligned stimuli. In addition summation of the uniform stimuli followed more closely a fourth ront summation rule (sensitivity $S \geq N^{1 / 4}$ ) than the random stimuli, as was shown before for contour integration (Bonneh and Sagi, 1998). 2) Cueing did not affect contrast detection thresholds or spatial summation. This result is consistent with previous studies showing that cuing the location of a target that is presented without masks does not affect its contrast detection threshold (Smith, 2000; Zenger et al., 2000; Foley and Schwarz, 1998; Solomon et al., 1997). This observation suggests a pre-attentive, parallel processing of the visual field and is incompatible with uncertainty model s (Pelli, 1985). It also shows that the excitatory lateral interactions between the spatial filters are not affected by attention when activity is low.

\section{Altered anticonvulsant drug sensitivity of hippocampal} neurons in chronic epilepsy

Schaub C., Remy S. and Beck H.

Dept. of Epileptology, University of Bonn, Germany

The development of pharmacoresistance to antiepileptic drugs in patients with epilepsy may rely on reduced drug sensitivity of target molecules. In the case of carbamazepine (CBZ) and phenytoin (PHT), this notion is supported by a loss or reduction of use-dependent block of voltage-dependent $\mathrm{Na}^{2}$ currents in hippocampal granule neurons following epileptogenesis. We have examined whether a similar loss of efficacy occurs also in other hippocampal cell types, namely CA1 pyramidal neurons.

In control CAl neurons, CBZ $(100 \mu \mathrm{M})$ and PHT $(100 \mu \mathrm{M})$ significantly shifted the voltage-dependence of fast inactivation towards more hyperpolarized potentials (CBZ: $9.0 \pm 0.6 \mathrm{mV}$; PHT: $6.7 \pm 1.1 \mathrm{mV}, \mathrm{p}<0.05$ ) and the activation curve in the depolarized direction (CBZ: $2.4 \pm 0.5$; PHT: $2.5 \pm 1.5$ ). Both effects were unaltered in pilocarpine treated rats (Inactivation: $\mathrm{CBZ}$ : $8.6 \pm 0.4 \mathrm{mV}$; PHT: $10.6 \pm 1.2 \mathrm{mV}$; Activation: $\mathrm{CBZ}: 3.1 \pm 1.1$; PHT: $3.7 \pm 0.7$ ).

Next, we compared the drug effects on the biexponential time course of fast recovery from inactivation (time constants $t_{\text {slow }}$ and $\left.t_{\text {rass }}\right)$. CBZ induced a profound slowing of the recovery, expressed mainly as a significant increase of the amplitude proportion of the slower time constant $\left(A_{\text {slow }}\right)$ and increase in $t_{\text {fast }}$ while $t_{\text {slow }}$ even decreased ( $t_{\text {slow }}: 0.97 \mathrm{~s}$ to $0.61 \mathrm{~s} ; \mathrm{A}_{\text {slow: }}: 0.27$ to $0.55, \mathrm{p}<0.05$ ). In pilocarpine-treated rats, $\mathrm{CBZ}$ did not exert significantly different effects on the time constants, unlike prior data in granule neurons, but was less effective in altering $A_{\text {slow }}\left(A_{\text {slow }}: 0.35\right.$ to $\left.0.56, p<0.05\right)$. PHT also induced a slowing of the recovery in both groups, with an even larger effect on the amplitudes and increases in both $t_{\text {slow }}$ and $t_{\text {fast }}$ (Control: $t_{\text {siow }}: 0.87 \mathrm{~s}$ to $1.15 \mathrm{~s}: \mathrm{A}_{\text {siow }}: 0.27$ to 0.74 ; Pilocarpine: $t_{\text {infy }}: 0.93 \mathrm{~s}$ to $1.13 \mathrm{~s} ; \mathrm{A}_{\text {slow }}: 0.33$ to 0.74 ) with no significant differences between the two experimental groups.

Our results point to a subregional difference in anticonvulsant drug effects between the dentate gyrus and the CAl region of the hippocampus. 
Scanning the voltage sensor of KCNQ1 potassium channels: A puzzling portrait of channel gating Schottelndreier H., Shamgar L., Peretz A. and Attali B. Dept. of Physiology and Pharmacology, Sackler Medical School, Tel Aviv University, Tel Aviv

The KCNQ potassium channels whose mutations cause cardiovascular and neurological disorders are members of the large superfamily of voltage-gated $\mathrm{K}^{+}$channels, sharing the $\mathrm{S} 4$ voltage sensor sequence. Movement of the S4 segment induces a conformational change that opens the channel pore. The KCNQ1 pore-forming subunit can interact with various auxiliary $\mathrm{KCNE}$ protein subunits to form $\mathrm{K}+$ channels with very different gating behaviors. As an attempt to characterize the structural basis of the promiscuous gating of KCNQ1 channels, we performed a tryptophan-scanning mutagenesis of the KCNQ1 S4 sensor and analyzed the mutation-induced perturbations in gating free energy. Our data indicate that specific $\mathrm{S} 4$ residues bias $\mathrm{KCNQ} 1$ channels toward either the inactivated state the open state or the closed state, while others totally prevent channel closure. The energetic impact on many mutated residues do not accommodate with a voltage sensor interfacing with the membrane lipids at the channel periphery. Perturbing the gating energetics of KCNQ1 recapitulates many of the promiscuous gating behaviors induced by co-assembly with auxiliary $\mathrm{KCNE}$ subunits

Hypoxia inducible factor $1 \alpha$ and erythropoietin receptor upregulation in the brain of heat acclimated mice following closed head injury: possible role in neuroprotection closed head injury: possible role in neuropr

Depts. of Pharmacology and Physiology ${ }^{2}$, Hadassah Schools of Pharmacy and Dental Medicine, the Hebrew University

\section{rusalem}

Background: Long-term exposure to heat (heat acclimation, ACC) was shown to protect against brain damage after closed head injury (CHI) in rats (Restor Neurol \& Neurosci 6:107, 1994). Hypoxia inducible factor 1 (HIF1), a transcriptional activator, is essential for the activation of various genes including erythropoietin (Epo), erythropoietin receptor (EpoR), some glucose transporters and glycolytic enzymes, all of which can potentially play a role in promoting cell survival. Oxidative stress, occurring within minutes after $\mathrm{CHI}$ is a possible HIF1 regulator. This study examined 1) whether ACC is also neuroprotective in mice and 2) whether ACC affects brain expression of HIF $1 \alpha$ and subsequently EpoR levels before, and at various times after injury Methods: Mice were held at ambient temperature of $24^{\circ} \mathrm{C}$ (controls) or $34^{\circ} \mathrm{C}$ (ACC) for 30 days (Basic Res Cardiol 98/3, 185) and then subjected to left cerebral hemisphere $\mathrm{CHI}$, by a weight drop device (under ether anesthesia), or sham surgery. Clinical recovery was evaluated by measuring the motor function and edema formation (\% water content) in mice $24 \mathrm{~h}$ after $\mathrm{CHI}$. Four and $24 \mathrm{~h}$ after surgery or $\mathrm{CHI}$, mice were sacrificed and their brains removed for western blot analysis of HIFla and EpoR. Results: Clinical recovery was markedly greater in ACC mice as compared to controls $(p<0.05)$ and edema formation was significantly smaller $(p<0.05)$. Following sham surgery alone, HIF $1 \alpha$ levels tended to be higher in ACC mice than in controls. CHI led at $4 \mathrm{~h}$ to an additional increase in HIF $1 \alpha$ levels in both groups, maintaining the difference between the two $(\mathrm{p}<0.05)$. Preliminary data also indicate that basal EpoR levels in ACC mice are higher. Discussion: Our findings show neuroprotective effects of ACC in mice. The causal evidence between better $\mathrm{CHI}$ recovery following $\mathrm{ACC}$, upregulation of HIFl and in turn its target gene EpoR may suggest its involvement in neuroprotection. It is noteworthy that Epo was shown to be neuroprotective in this model.

\section{Computerized geometrical methods for analysis of the \\ sleep}

\section{Shmiel O., Teicher M. and Dagan Y.}

Bar Ilan University, Ramat Gan

The Experiment: The research is performed with the cooperation of the sleep lab at "SHIBA" hospital under the supervision of Prof M. Teicher and Dr. Y. Dagan.

The lab diagnoses sleep disorders of patients by computerized sleep rooms which allow the connection of electrodes and sensors to the patient. Each electrode/sensor records a signal of a specific channel during the night (i.e. EEG, EKG, Pulse, etc...) on the same time scale, so that all channels are synchronized. Finally, when the patient awakens, the recorded data is analyzed.

Polysomnogram examination of this integrated data gives much information about the sleep of the patients, such as recognition of sleep stages, sudden arousals, breathing difficulties and other abnormal behavior. The analysis is performed simply by looking at the channels and has a major importance in the detection of sleep disorders and other pathologies.

Background: Sleep is divided to five stages that have a significant importance on the analysis of sleep disorders. The EEG channel has a major rule in most of the well known sleep analysis procedures today. Most of the sleep analysis methods are based on the frequency of this channel, its amplitude and known structures of it. Some of these methods are described in the poster, as well as other methods regarding different channels.

Goals: 1) Prediction of pre-sleeping behavior, using only channels which can be recorded easily. 2) Prediction of arousals, using channels which can also be recorded easily. 3) Finding relationships between the GSR and other channels.

Method Description: Primarily, every channel is translated into relevant basic components. Then, repeating component patterns are detected by data mining algorithms. Finally, confrontations between the patterns are performed in order to find statistically surprising phenomena.

In addition, confrontations between patterns and human analysis are performed in order to find relationships between these patterns and the known analyzed subconscious activities.

Using data-mining techniques to reveal structure of drawing and brain activity

Shmiel T., Drori R., Ben-Shaul Y., Teicher M. and Abeles M.

Bar Ilan University, Ramat Gan

The main goal of the research was to examine relationships between the drawing behavior and the single unit activities that may be revealed by data-mining techniques.

While the direction of motion is traditionally considered to be coded in the firing rates of the units, this research focuses on finding relationships involving only synchronized spike events from the brain activity.

Activity from motor and premotor areas of two monkeys was recorded while the monkeys performed continuous drawing movements in the horizontal plane. The main method of analysis used was transforming both brain and hand activities to some other languages, which are more concise. These languages summarized the basic events in each activity, and were used later as the basis for all the revealed relationships. After this transformation, data-mining algorithms were applied to the translated data in order to find components repeating in each data independently. Finally, components from the two kinds of data were confronted (using a variety of statistical methods), and statistically unexpected relationships were searched.

Supported in part by ISF.

The whole may be perceived faster than its parts

Shneor E. and Hochstein S

Neurobiology Dept., Institute of Life Sciences and Neural Computation Center, Hebrew University, Jerusalem

Subjects detect rapidly and pre-attentively the presence of an odd element that differs greatly from surrounding elements in a single dimension, without knowing it's specific location. We now ask what will be the speed and accuracy of detecting 2 such elements, simultaneously. Subjects viewed a briefly presented $8 \times 8$ array of pink lines oriented at $55^{\circ}$ (or $60^{\circ}$ ) followed by a masking stimulus after a variable Stimulus-to-mask Onset Asynchrony. On some trials, 1 or 2 of the elements were replaced by a pale green line of the same orientation, a pink line of a different orientation $35-40^{\circ}$ (or $30^{\circ}$ ), or a line with both these changes. Subjects reported the number of odd lines $(0,1$ or 2$)$ and their nature (orientation and/or color difference). Surprisingly, we found that subjects were more accurate at detecting and discriminating targets in arrays with 2 odd elements than arrays with one, no matter the type of the odd elementls. We calculated detectability, d' according to Single Detection Theory (SDT), and found that $\mathrm{d}^{\prime}$ for detection and identification of 2 odd elements is higher than for 1 element. Consequently, people don't have a 'favorite guess' but really find detection/ identification of 2 odd elements is better! In addition, we found that it was easier to report the presence and type of two different dimensions when they were located at different places in the array. We conclude that detection and identification take place at high cortical levels, perhaps at Inferior Temporal cortex, where arrays with a pair of targets are represented as distinct unitary structures, as suggested by Reverse Hierarchy Theory (Hochstein \& Ahissar, Neuron, 2002).

Supported by an Israel Science Foundation "Center of Excellence" grant and the US-Israel Binational Science Foundation (BSF). 
Memory deficit induced by intracerebroventricular streptozotocin in rats is associated with oxidative stress, septohippocampal damage and impaired energy

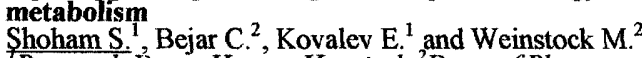

${ }^{T}$ Research Dept., Herzog Hospital, ${ }^{2}$ Dept. of Pharmacology, Faculty of Medicine, Hebrew University, Jerusalem

One of the earliest detectable abnormalities in Alzheimer's disease (AD) is a decrement in cortical energy metabolism in the cortex and hippocampus. This can be induced in rats by intracerebroventricular injection of streptozotocin (icv-STZ) We have previously shown that icv-STZ causes specific degeneration of periventricular myelinated structures including the fornix, resulting in damage to the septohippo-campal cholinergic projection and in spatial memory deficits. The present study determined whether bilateral icv-STZ $(0.5 \mathrm{mg})$ in 3-month old rats caused oxidative stress prior to myelin damage. Oxidative-nitrative stress was detected by immuno-histochemical staining of nitrotyrosine (NT), formed from nitration of tyrosine by peroxynitrite, a highly reactive derivative of NO and $\mathrm{O}_{2}^{-}$. One week after icv STZ, rats were anesthetized and brains fixed by transcardial perfusion with paraformaldehyde. A loss of astrocytes occurred in areas injection, while NT staining was seen in surviving astrocytes in the perimeter of these areas. Double staining by rabbit anti-NT and mouse anti-glial fibrillary acidic protein confirmed that NT was expressed in astrocytes in these structures as well as in astrocytes in the corpus callosum, lateral septum and fornix. Damage to myelin was observed in the same structures 5 weeks later, suggesting that that oxidative-nitrative stress in astrocytes induced by icv-STZ precedes and probably contributes to myelin damage. Nitration of tyrosine and damage to myelin also occur in the brains of AD patients in addition to deficits in memory and glucose utilization. The icv injection of STZ in the rat could therefore serve as an experimental model for the development of antioxidant and other neuroprotective medications for the treatment of conditions in which demyelination is involved. Supported by the Magnet Program of the Israeli Ministry of Trade and Industry

Stereotypic behavior in transgenic FVB/N mice with AChE-R over-expression: Evidence for cholinergic and dopaminergic contributions Shoham S. ${ }^{1}$, Kovalev E. ${ }^{1}$, Cohen O. $^{2}$, Yirmiya R. ${ }^{3}$, and Soreq H.

${ }^{I}$ Research Dept. Herzog Hospital, and Depts. of ${ }^{2}$ Biological Chemistry, and ${ }^{3}$ Psychology, Hebrew University, Jerusalem Israel

Spontaneous, drug-independent stereotypic behavior (SB) has been documented in several inherited syndromes (e.g. autism, schizophrenia), however, the neurotransmission circuits controlling this phenomenon are yet unknown. Here, we report the emergence of SB by $7-10$ weeks of age in TgR mice overexpressing, under cytomegalovirus promoter regulation, the stress-induced variant of human acetylcholinesterase hAChE-R. SB included repetitive movements, increased locomotion time, locomotor asymmetry, and reduced rearing in open field, but with preservation of circadian motor activity cycle. Immunohistochemical mapping revealed accumulation of $h A C h E-R$ in cholinoceptive regions including hypothalamic and brainstem locomotor regions, but cholinergic and dopaminergic neurons generally did not accumulate hAChE-R (except for a subset of cholinergic neurons in the nucleus basalis magnocellularis). The distributions of cholinergic and dopaminergic neurons were normal, with inter-hemispheric symmetry of dopaminergic and cholinergic neurons. However, ÁChE-R labeling in hippocampal CA1-3 pyramidal neurons was inversely associated with asymmetry and hyperlocomotion, indicating that $\mathrm{AChE}-\mathrm{R}$ accumulation in both hippocampal and other behaviorally inhibitory limbic brain regions moderates $\mathrm{SB}$. In $\mathrm{TgR}$ mice, haloperido (dopamine antagonist) at $0.5 \mathrm{mg} / \mathrm{kg}$ reduced locomotion and attenuated motor asymmetry in TgR mice, whereas apomorphine (dopamine agonist) at $0.1 \mathrm{mg} / \mathrm{kg}$ induced abnormal slow locomotion and did not affect motor asymmetry. Also, pilocarpine, a cholinergic muscarinic agonist, increased the accumulation of hAChE-R in hippocampal CA1-3 neurons while reducing accumulation in hypothalamic and brainstem locomotor regions and suppressing locomotion. These findings implicate both dopaminergic neurotransmission and neuronal distribution of AChE-R in disorders with adult onset SB, such as schizophrenia.
Cognitive impairments and negative symptoms in schizophrenia: Core dysfunctions and response to treatment

Silver $H$.

Brain Behavior Laboratory, Sha'ar Menashe Mental Health Center, Hadera, and Bruce Rappaport-Faculty of Medicine, Technion Institute of Technology, Haifa

Multiple cognitive and emotional impairments are prominent features of schizophrenia which respond poorly to treatment and retard rehabilitation. It is a goal of schizophrenia research to identify unifying, core mechanisms which can parsimoniously explain such phenomenological diversity and provide new targets for treatment. We studied relationships between diverse neuropsychological functions and symptoms in schizophrenia patients, aiming to identify such core deficits and examine their response to treatment. Data will be presented indicating that working memory (WM) dysfunction may be a core deficit in schizophrenia such that the reduced capacity of verbal and spatial "on-line storage" is rate limiting in performance of other cognitive functions. WM deficits are associated with negative symptoms suggesting use of common circuits in the PFC. Treatment with SSRI added to antipsychotics led to improvement in some but not all negative symptoms. In key primary negative symptoms including affective flattening and alogia improvement could be detected within 2-3 weeks of treatment onset. Our findings support the hypothesis that diverse cognitive and emotional deficits in schizophrenia result from impairment in a limited number of core dysfunctions. These may reflect a functional, biochemically mediated, imbalance rather than static brain damage and may be amenable to pharmacological interventions.

\section{Confocal imaging of mRNA transport, localization and} EGFP tagged protein translation in cultured Aplysia neurons

Slonimsky G., Shapira E. and Spira M.E

Dept. of Neurobiology. Life Sciences Institute the Hebrew University of Jerusalem.

Translation of proteins in "remote" neuronal compartments appears to play important roles in housekeeping and neuroplasticity. Presently, the transport, targeting, and control mechanisms of mRNAs at remote sites are mostly studied in fixed cells.

Here we examine the potential use of in vitro translated fluorescently labeled mRNA (fmRNA) that encodes EGFP tagged proteins to image on line the distribution of $\mathrm{fmRNAs}$ and their translational products. To that end we in vitro synthesized fmRNA encoding EGFP actin using protocols developed by our laboratory. The fmRNA was synthesized using Alexa 546- dUTP's and injected to the cytosol of cultured Aplysia neurons. Its spatiotemporal distribution was imaged by confocal system. Consistent with earlier studies we found that fmRNA encoding EGFP-Aplysia-actin is translated in isolated axonal segment. Injection of the fmRNA into the cell body cytosol revealed that fmRNA translocates into the axon forming a gradient in which the fmRNA intensity was greatest in the soma. In the axon the injected fmRNA appears in granules and elongated structures. A fraction of the granules are stationary while others propagated in both directions along the axon at a rate of $0.1-0.29 \square \mathrm{m} / \mathrm{sec}$. The EGFP-actin translational product distribution accompanied the moving front of the fmRNA.

Axotomy lead to the concentration of fmRNA in the growth cone's (GC) center. When a GC's lamellipodium extends from this center, typical EGFP-actin bundles are formed along the edges of the lamellipodium. EGFP-actin assembly occurs 10-20 \m away from the fmRNA localization site at the GC's center. As microtubules invade the GC's lamellipodium, the fmRNA translocates centripetally to concentrate in newly formed secondary GCs. These observations were reproduced by FISH. Interestingly, the full length form of Aplysia $\square$-actin mRNA and that which lacks the 3' UTR, behave similarly.

Supported by the BSF.

\section{Context dependant activity in primary olfactory cortex of}

humans

Helen Wills Neuroscience Institute, UC Berkeley, Berkeley, CA Attentional modulation of neural activity patterns has been demonstrated in primary visual and auditory cortex. Activity throughout the olfactory system is also context dependant. Activity in the rat olfactory bulb reflects cues that are merely associated with odor content, and activity in rat olfactory cortex reflects the motivational significance of an odor. To ask whether contextual modulation is evident in activity patterns of human primary olfactory cortex we used functional magnetic resonance imaging to measure sniff-induced neural activity in and out-of an olfactory context. Each trial of an event related 
design began with an auditory primer for "task detection" or "task inhalation". In "task detection" subjects took one sniff and determined whether an odorant was present or not. Odor was present on half of these trials. In "task inhalation" subjects also took one sniff but knew in advance that an odor would never be present. Thus, the only difference between sniffs in "task inhalation", and the no-odor sniffs of "task detection" was that in the latter condition subjects were exploring for the presence of odor. Real-time measurement of nasal respiration assured sniffs were equal across conditions. Twelve subjects were scanned at 4 T $(2$-shot T2* sensitive EPI, TR $=1000 \mathrm{~ms}$, $\mathrm{TE}=30 \mathrm{~ms}$, flip angle $=20^{\circ}, 64 \times 64$ voxel matrix, $192 \times 192$ $\mathrm{mm}$ FOV, in-plane resolution $=3.5 \mathrm{~mm}$, through-plane resolution $=3.5 \mathrm{~mm}$ ). Activity in primary olfactory cortex was significantly higher when sniffing clean air in "task detection" than when sniffing the same content in "task inhalation" $(p<$ $.04)$. In other words, activity in human primary olfactory cortex was strongly dependant on task context.

\section{$M-c u r r e n t s$ are reduced in CA1 pyramidal neurons of} chronic epileptic rats

Sochivko D. ${ }^{\text {, Chen J. }}{ }^{1}$, Becker A. ${ }^{2}$, Yaari Y. ${ }^{3}$, and Beck H. ${ }^{1}$ Depts. of Epileptology and ${ }^{2}$ Neuropathology, Univ. of Bonn Medical Center, Germany; ${ }^{3}$ Dept of Physiology, Hebrew

Univ., Israel

M-currents belong to the group of voltage-dependent potassium currents. They are characterized by slow kinetics and operate at the voltages near action potential threshold. Due to these properties, M-currents contribute to the control of neuronal excitability and firing behavior. M-currents are supposed to be mediated by KCNQ channels family. Recently, mutations of the genes encoding KCNQ2 and 3 channel subunits were shown to underlie, a form of inherited epilepsy in human. It is known that intrinsic firing properties of CAI hippocampal pyramidal neurons are modified in the chronic epileptic condition developing after single episode of status epilepticus (animal model of temporal lobe epilepsy). In the view of possible role of $M$-currents in such changes, we analyzed expression of M-currents in CAl neurons at different stages of model epileptogenesis using whole-cell patch clamp technique in hippocampal slices. M-currents were isolated by application of specific blocker linopirdine $(10 \mu \mathrm{M})$. In the early epileptic rats (7-14 days after pilocarpine-induced status epilepticus), the maximal density of $M$-currents was not
changed $(1.36+1-0.14 \mathrm{pA} / \mathrm{pF})$ when compared with the control group $(1.1+1-0.06 \mathrm{pA} / \mathrm{pF})$. In the late epileptic animals $(30-100$ days after status epilepticus), M-current density amounted to $0.74+1-0.12 \mathrm{pA} / \mathrm{pF}$ and was significantly reduced when compared with control $(1.53+1-0.27 \mathrm{pA} / \mathrm{pF}, \mathrm{p}<0.05)$. This reduction was especially prominent at physiologically significant voltages (to $26 \%$ of control value at $-43 \mathrm{mV}$ ). These results suggest that switch in the firing properties of $\mathrm{CAl}$ neurons from regular spiking to bursting in the chronic epileptic conditions may be due to the down-regulation of M-currents which makes these currents a potential target of antiepileptic drugs.

The use of virtual reality for integrative multi-modal testing, and application in schizophrenia

Sorkin A. ${ }^{1}$, Peled A. ${ }^{2}$ and Weinshall D. ${ }^{1}$

Interdisciplinary Center for Neural Computation, Hebrew

University, Jerusalem: ${ }^{2}$ Institute for Psychiatric Studies,

Sha'ar Menashe MHC, and the Rappaport Faculty of

Medicine, Technion, Haifa.

Schizophrenia is a very debilitating disease; recent insights into the brain pathology of schizophrenia suggest that disturbances in multiple parallel processing in the brain may be responsible for the disease. Since the problem is cross modal and integrative, we advocate the use of Virtual Reality Technology (VRT) for diagnosis and testing. This technology would allow us to study brain functions in parallel, and the integration among them. VRT will also allow us to measure simultaneously all the interactions of the patients with the simulated environment. To our best knowledge it is the first report of schizophrenia study using VRT.

Methods: We have completed one VRT study testing sensory integration within working memory. Specifically, subjects navigate in a VRT maze, passing through multiple rooms with 3 doors each. The doors are characterized by three features: color, shape and sound. At any given moment there is a rule controlling door opening based on a certain combination of these features. The subject needs to learn the rule and to use it for navigation through the maze. The rule changes over time, without any notification to the subject.

Results: In analyzing errors rate we measured the effect of two factors: (i) the presence of a distractor (a feature that doesn't affect the door opening rule), and (ii) the complexity of the rule (i.e., the number of features determining the rule). In the most complex condition, when the rule was determined by 2 features and one distractor, schizophrenic patients were adversely affected by the distractor. Surprisingly, we did not see decreased ability among patients to adjust to rule changing. commonly referred to as perseveration which is a known deficiency in schizophrenia. Overall schizophrenic patients did not differ from the controls on most parameters studied such as game strategy and exploration of the environment. Patients differed from the controls by overall slower performance and increased errors rate.

The dependency of the acquisition of geometrically defined motion elements on visual feedback and on the implementation of smooth motion strategy Sosnik R. ${ }^{1}$, Hauptmann B. ${ }^{2}$ Karni A. ${ }^{1,3}$ and Flash T. Teizmann Inst. of Sci., Rehovot Israel; ${ }^{2}$ Dept. of Neurology Charit10098,'Berlin, Germany: ${ }^{3}$ The Brain-Behovior Research Center, University of Haifa, Haifa, Israel

We previously showed that extensive training on a sequence of planar hand trajectories passing through several targets resulted in the co-articulation of movement components and in the formation of new movement elements (primitives) (Sosnik et al 2001). Reduction in movement duration was accompanied by the gradual replacing of straight trajectories by longer curved ones, the latter affording the maximization of movement smoothness ("global motion planning"). The results from transfer experiments, conducted by the end of the last training session, have suggested that the subjects have acquired geometrically defined movement elements, which were solely dictated by the figural (i.e., geometrical) form of the path, rather than by both path geometry and the temporally dependent velocity profiles. Here we show that only $28.5 \%$ of the participants (two out of seven) who were trained in the absence of visual feedback (dark condition) co-articulated by the end of the last training session compared to $75 \%$ (eight out of twelve) who were trained on the same target configuration in the light. Furthermore, no geometrical motion elements were acquired while training in the dark. Surprisingly, in the light condition, the emergence of geometrically defined motion elements was temporally correlated with the implementation of the "global motion planning" strategy. Our results indicate that visual feedback of the hand has strong effect on the capability to implement the "global motion planning" strategy and on the acquisition of geometrically defined motion elements in adults. Supported by Dominic-Einhorn Fund.

\section{Real-time imaging of Golgi derived vesicle exocytosis} during the formation of growth cone lamellipodium after axotomy of cultured Aplysia neurons

Spira M.E. and Malkinson G

Life Sciences Institute, The Hebrew University of Jerusalem

The transformation of a stable axonal segment into a motile growth cone is a critical step in the regeneration of amputated axons. Axotomy of cultured Aplysia neurons is followed by rapid extension of a growth cones (GC) lamellipodium. Calculations revealed that to account for this rate of growth, 80-180 vesicles/sec fuse with the plasmalemma.

Here we began to explore where, when and how Golgi derived vesicles fuse with the plasma membrane in support of the growth process. To that end, we expressed the $\mathrm{pH}$-sensitive GFP fused to the luminal domain of VAMP synapto-pHluorin (a gift from J. E. Rothman, Memorial Sloan-Kettering Cancer Center, NY) in cultured neurons. Detection of vesicles distribution before, during and after axotomy as well as throughout the process of GC extension was done by confocal imaging using excitation wavelength of $488 \mathrm{~nm}$ to image the probe during or after fusion of the labeled vesicles, and by imaging of the total population of synapto-pHluorin labeled vesicles with excitation wavelength of $405 \mathrm{~nm}$. Calcium was imaged by intracellular injection of Fluo-4 or Rhod -2. A fraction of these indicators is taken up by the vesicles and reveals relatively high intravesicular calcium levels.

We found that axotomy leads to: (a) Fusion of anterogradlly transported vesicles with the plasma membrane at the tip of the axon where the $\left[\mathrm{Ca}^{2+}\right]_{i}$ is elevated to $>1 \mathrm{mM}$. (b) After the recovery of the $\left[\mathrm{Ca}^{2+}\right]_{i}$ to control, Synapto-pHluorin labeled vesicles accumulate at the forming GC's center. (c) These vesicles fuse with the GC's plasma membrane in the vicinity of the GC center. The fusion process proceeds in the absence of any detectable fluctuations in the $\left[\mathrm{Ca}^{2+}\right]$

In conclusion, the addition of new membrane to axotomyzed axon is exclusively localized to the GC center. We propose that the preferential fusion of vesicles at the GC center is facilitated by release of calcium from the vesicles themselves and their local concentration

Supported by: The ISF and the BSF 
TIEG2 induces apoptosis in the oligodendroglial cell lineage Oli-neu

Spittau B., Peters B., Krieglstein K. and Wang Z.

Dept. of Neuroanatomy, Gottingen, Germany

Apoptosis is one of the most important incidences during the development of neurons as well as oligodendrocytes. The induction of apoptosis is mediated by various cytokines, one of them is the multifunctional growth factor TGF- $\alpha$. Our previous work has revealed that TGF- $\alpha$ induced cell death is mediated via down regulation of the anti-apoptotic protein $\mathrm{Bcl}-\mathrm{X}_{\mathrm{L}}$ and activation of caspase 3 . Here we address the question which downstream signals regulate the transcription of TGF- $\alpha$ target genes. The candidate molecule is TIEG (TGF- $\alpha$ immediate early gene), a newly defined subfamiliy of the $\mathrm{Sp}-1$ transcription factors. TIEG proteins bind to the DNA with their zinc finger motif and act as a transcriptional repressor. Over expression of TIEG1 is sufficient to induce apoptosis in many cell types, whereas TIEG2 has been shown to inhibit cell growth. In this study we show that the transient expression of TIEG 2 can induce a programmed cell death in Oli-neu cells. The detection of apoptotic cells was accomplished using the TUNEL assay and an apoptosis ELISA. Furthermore, we could demonstrate that TIEG2 functions by repressing the anti-apoptotic protein, $\mathrm{Bcl}-\mathrm{X}_{\mathrm{L}}$, whereas $\mathrm{Bcl} 2$ remained unchanged. Together, our finding suggests that TIEG serves a general role in TGF- $\alpha$ induced apoptosis, bridging from TGF- $\alpha$ dependent signaling to the acknowledged intracellular pathway of apoptosis.

Biological gain control for high dynamic range

Spitzer H., Karasik Y. and Einav S.

Dept. of Biomedical Engineering, Faculty of Engineering,

Tel-Aviv University, Tel-Aviv

The visual system has the ability to see and get detailed information from high dynamic range scene. For example, a person can observe items in a one sight while observing in a dim room and outside through a window. A model and an algorithm were developed for high dynamic range compression that expose the details in an image, for example, as occurs often in images where there are bright and dark areas where these details are appeared as lost. The model succeeds in automatically compressing the dynamic range of images to a 'human vision appearance' (as is commonly required in cameras and displays) while maintaining contrast and even improving it. The model is based on biological retinal mechanisms of adaptation (gain control): 'local', and 'remote' This model can explain also the lightness constancy. These mechanisms enable also video image applications, since they take into account the dynamics of human adaptation mechanisms. The results indicate that the contribution of adaptation mechanisms to image appearance is significant robust, and was proved to fit next generation High dynamic range cameras (CMOS based). The algorithm was tested on images with standard format $\left(2^{8}\right.$ bits $)$ and HDR $\left(\sim 2^{8}\right.$ bits). The algorithm started to be applied to: real photographs, medical images such as ultrasound images satellite images.

What are the roles of insular cortex in taste processing and memory?

Stehberg J., Desmedt A. and Dudai Y

Dept. of Neurobiology, The Weizmann Institute of Science, Rehovot.

The insular cortex is considered to contain the primary cortical area for taste processing. Yet numerous studies, using lesions and pharmacological interventions, have not yet yielded conclusive evidence concerning the precise role of the insular cortex in taste functions in general and in taste learning and memory in particular. In a set of studies, we aimed to define the role of the insular cortex in taste-guided behavior and memory, by subjecting rats with a bilateral ibotenic acid ablation of the insular cortex to a spectrum of behavioral paradigms. These experiments targeted the role of the insular cortex in taste perception, neophobia (i.e. fear of the new) familiarity acquisition, novelty-familiarity discrimination, and conditioned taste aversion (CTA). In CTA, we tested distinct memory phases, including acquisition, retention, retrieval, and experimental extinction. We also tested the potential role of cortical laterality in the aforementioned functions. Our data suggest a role of the insular cortex in the CS-US association in CTA, but not in taste perception per se. Among others, our data suggest that animals lacking insular cortex and subjected to CTA training, can still remember the US experience (malaise) but cannot associate it with a specific taste. Instead they might subsequently beware of drinking novel tastes in general. These and other results give rise to a novel anatomical and functional model for the central taste system. Supported by the Israel Science Foundation.
The many functions of herpes simplex virus type 1 latency associated gene

Steiner I., Mador N., Braun E., Haim H. and Panet A. Laboratory of Neurovirology Dept. of Neurology \& Dept. of Virology, Hadassah University Hospital

Jerusalem.

Objectives: To study the function(s) of herpes simplex virus type 1 (HSV-1) latency-associated gene (LAG).

Background: The LAG is the only gene expressed during HSV-1 latent infection in human peripheral sensory ganglia. It has been linked to viral reactivation and we have shown that it suppresses viral replication in neuronal cells (Mador et al., $J$. Virol., 1998)

Methods: In order to further facilitate the study of the LAG function we have generated a LAG-expressing transgenic mouse and assayed replication of a LAG-negative HSV-1 mutant in transgenic mouse embryonic fibroblasts (MEFs). The virus was also used to establish latent infection in trigeminal ganglia (TG) and measure reactivation efficiency. Using real-time quantitative polymerase chain reaction (PCR) was used to measure viral DNA in TG.

Results: The mutant replication was limited in transgenic MEFs. This was not due to a toxic effect of the LAG upon cell. Reactivation of the virus from TG was improved by three different parameters: reactivation ability $(100 \%$ vs. $75 \%)$; reactivation kinetics (maximal reactivation was achieved at day 7th post explantation compared to day 11th in controls) and an increased titer of reactivated virus. HSV-I genome was identified in reactivation-negative ganglia. Using real-time quantitative polymerase chain reaction we found that latently infected TG from transgenic mice did not contain increased amounts of HSV-1 DNA.

Conclusions: The LAG may provide several of the functions required for HSV-1 latent infection in peripheral sensory ganglia: enabling the virus to enter the latent state, facilitating reactivation and protection of the viral reservoir.

\section{Bidirectional communication between rhythmogenic} networks in the neonatal rat spinal cord Strauss I. and Lev-Tov A.

Dept. of Anatomy \& Cell Biology, The Hebrew University Medical School, Jerusalem

The ability of neuroactive compounds to induce coordinated rhythmic-patterns in limb- and non-limb inmervating segments of the spinal cord was examined in isolated spinal cords of neonatal rats. Addition of NMDA/5HT to the cord or selective application of NMDA/5HT to the thoracolumbar (TL) segments induced locomotor-like activity accompanied by alternating left-right sacrococcygeal (SC) thythm. The TL and SC activities were coupled in the rostrocaudal direction. Transecting the cord at the lumbosacral junction nearly blocked the SC rhythm and did not interfere with the TL rhythm. Specific lesions of the white matter funiculi revealed that the rostrocaudal coupling was obtained by pathways traveling through the ventral $(\mathrm{VF})$, ventrolateral (VLF), and lateral (LF) funiculi.

Bath application of the alphal-adrenoceptor agonist methoxamine to the cord or selective application of methoxamine to the SC segments induced a fast alternating left-right rhythm in the SC and TL segments. The fast TL rhythm appeared only in flexor dominated segments; it was entrained by the SC rhythm, and abolished in surgically-detached TL cords. Specific lesions of the VF virtually abolished the caudorostral coupling between the SC and TL segments during the rhythm, but did not impair the SC rhythm.

In summary, the TL and SC rhythmogenic networks can be selectively activated by different neurochemicals; rostrocaudal coupling between them via the VF, VLF and LF ensures a coordinated activation of the limb, axial and tail musculature; and caudorostral coupling via the VF allows modulation of activity in flexor-dominated segments of the TL cord during the methoxamine-mediated rhythm.

Putative intracellular targets of lipophilic divalent metal ion chelators in attenuating MMP-9 activity in glial cells Striem S. Gileadi C., Schatz G., Meirav T., Friedman J.E., Angel I., Kozak A. and Haring R

D-Pharm Ltd., Rehovot, Israe?

The Matrix Metalloproteinases (MMPs) are extracellular calcium- and $\mathrm{Zn}$-dependent proteases whose activation results in degradation of the extracellular matrix. Excessive expression of MMPs has been implicated in many CNS diseases involving inflammatory responses such as: Stroke MS, ALS, Alzheimer's and Parkinson's diseases. MMP-9 activity is determined by gene expression and pro-enzyme 
kinases (ERK, JNK and p38), protein kinase $\mathrm{C}$ (PKC) and $\mathrm{NF}-\mathrm{kB}$. We have synthesized a family of lipophilic metal chelators, DP-b99 and DP-109 that, in the cell-membrane milieu, preferentially bind zinc and copper compared to calcium. In the present study we have tested the hypothesis that lipophilic metal chelators can attenuate MMP-9 activity. DP-109 attenuated TNF $\alpha$-induced MMP-9 release and activity in A-172 human glioma cells, and reduced TNF $\alpha$ - and PMA-induced MMP-9 induction in C6 rat glioma cells, with an IC50 of $10 \mu \mathrm{M}$. DP-b99 (10-25 $\mu \mathrm{M})$ also reduced TNFo-induced MMP-9 activity Pretreatment with $50 \mu M$ $\mathrm{ZnCl}_{2}$ reduced the effects of DP-b99 and DP-109 on MMP-9 activity, demonstrating a zinc dependency. Activation of MMP-9 in C6 cells by TNF $\alpha$ and PMA was attenuated by PD98059 and U0126 (MEK inhibitors) and by GF109203X but not by SB203580 (p38 inhibitor), indicating involvement of ERK- and PKC-mediated pathways. Activation of ERK by

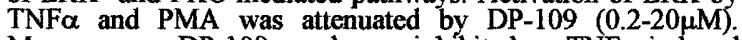
Moreover, DP-109 also inhibited TNFa-induced phospho-c-jun activation as well as NF-kB p65 nuclear translocation, indicating JNK and NF- $\mathrm{KB}$ as additional targets for DP-109. These findings suggest that DP-b99 and DP-109 can modify the activity/release of MMP-9 both directly and via affecting upstream targets. The ability of DP-b99 and DP-109 to attenuate activation of MMP-9 indicates the usefulness of these compounds in treatment of neurodegenerative disorders and correlates well with the protective effect of DP-b99 in the rat MCAO model and in human stroke, as previously reported. Support Contributed By: D-Pharm Ltd

The effect of DP-b99, a lipophilic divalent transition metal chelator, on Matrix Metalloproteinases- 2 and -9 activity in a rat MCAO ischemic model

Striem S., Schatz G., Krakovsky M., Finkelstein E., Kozak A., Angel I. and Friedman J.E.

D-Pharm Ltd., Rehovot, Israel

The Matrix Metalloproteinases (MMPs) are extracellular calcium- and $\mathrm{Zn}$-dependent proteases which are produced in a latent form and require activation for catalytic activity. Activation occurs at the cell surface and enables MMPs to degrade components of the Extracellular Matrix (ECM) at specific sites in the surrounding cell membrane. MMPs play important roles in a wide range of disorders such as brain injury and ischemia, Alzheimer's disease, inflammation and neuroinflammation, cancer, angiogenesis and cardiovascular diseases. MCAO/reperfusion injury is associated with increased MMP-9 activity correlated with a reduction in the major basal lamina constituents and brain edema. DP-b99, a lipophilic divalent transition metal chelator reduces TNFD induced MMP-9 activity in C6 glioma and in primary glial cells in vitro. In vivo, DP-b99 was found to reduce infarct volume and improve neurological scores in rats following both transient and permanent $\mathrm{MCAO}$, with a therapeutic window of at least 6 hours. In this study we tested the hypothesis that the neuroprotective effect of DP-b99 is correlated with its effect on MMP-9 and MMP-2 activity in a tMCAO ischemic rat model. The neuroscore of each animal was tested and the animals sacrificed. Blood was taken for NSE measurements and the brains for determination of infarct size and MMP-9 and MMP-2 activity. MMP activity in the ipsi-and contra-lateral hemispheres were compared and correlated with the other parameters measured. Administration of DP-b99 resulted in a decrease in the both MMP-9 and MMP-2 activity, albeit with different dynamics. The DP-b99 reduction in MMP-9 activity was highly correlated with reduced infarct volume $\left(r^{2}=0.86\right)$ and improved neuroscore $\left(r^{2}=0.79\right)$ after 24 hours. MMP-2 reduction was also well correlated $\left(\mathrm{r}^{2}=0.79\right)$ with the decrease in infarct volume indicating different patterns of regulation by DP-b99 for MMP-2 and MMP-9. These data suggest that the improved outcome of MCAO rats following DP-b99 treatment could be due, in part, to attenuation of the inflammatory component of stroke.

Support Contributed By: D-Pharm Ltd.

Pathways for anti-nociception during barbiturate induced general anesthesia

Sukhotinsky I., Devor M.

Institute of Life Sciences and Center for Research on Pain, Hebrew University of Jerusalem

Microinjection of minute quantities of pentobarbital into a restricted region of the rat brainstem, the mesopontine tegmental anaesthesia area (MPTA), can induce a state of general anesthesia including atonia and failure to response to strong noxious stimuli (Devor and Zalkind, Pain 2001). To understand the neural circuitry associated with various elements of the anesthetic state we are investigating the connectivity of MPTA. MPTA sends projections to and receives them from, various telencephalic, diencephalic, brainstem and spinal regions. There is a direct projection to the spinal cord but this ends predominantly in the ventral horn, not in dorsal horn structures normally associted with anti-nociception. However, there is also a major descending projection to the rostral ventromedial medulla (RVM), a key relay component of the descending brainstem system that modulates nociception in the dorsal horn. Do MPTA neurons with descending brainstem projections indeed terminate on RVM cells that project to the spinal cord ? To clarify this we performed double-labeling experiments. Bulbospinal RVM neurons were retrogradely labeled with CBT microinjected in the lumbar spinal cord, while the anterograde tracer BDA was microinjected into the MPTA. At the light microscopic leve we found numerous MPTA projections in the RVM. Labeled axons frequently came into close apposition with retrogradely labeled spinally projecting RVM neurons, somata and proximal dendrites. Moreover, many of these formed single or multiple varicosities, structures that may represent points of synaptic contact, although electron microscopy is necessary to confirm this inference. The massive projection of MPTA neurons onto bulbospinal RVM neurons is a route whereby barbiturates, and perhaps other anesthetic agents, may suppress responsiveness to noxious stimuli during general anesthesia.

Motor control of the octopus' fętch movement Sumbre G. ${ }^{1}$, Flash T. ${ }^{2}$, Fiorito $\mathrm{G}^{3}$ and Hochner B. 'Dept. of Neurobiology, Hebrew University of Jerusalem ${ }^{2}$ Dept. of Applied Mathematics, Weizmann Institute of Science; ${ }^{3}$ Stazione Zoologica di Napoli, Italy.

Previously we have shown that the octopus uses a highly stereotypical movement to accurately bring an object to its mouth. This fetching movement involves a dynamic three-jointed articulated-like structure, which rotates until the object reaches the mouth. Here we report an EMG study showing that the part of the arm, which is involved in the articulated-like structure (from the base of the arm to the location of the object) actively contracts or stiffens; suggesting that the articulated part of the arm is actively controlled.

A positive linear correlation is revealed between the integrated EMG level and the articulated structure length but not with kinematic variables such as the movements' velocity or acceleration. This suggests that stabilizing the articulated structure against water perturbations might be the control strategy evolved to achieve high accuracy.

Preliminary results show that two EMG signals; one starting close to the distal joint and the other at the base of the arm, propagate toward each other. Moreover, lesion experiments demonstrate that the fetching movement involves higher brain centers (basal lobe), suggesting involvement of central control. In addition, we observed a temporal pattern, in which the formation of the distal joint is followed by the medial one. This findings suggest that the stimulus produced by the grabbed object may activate, via a central command, two waves of muscle contraction, which collide at midway, creating the medial joint. This scheme may explain the adjustable feature of the two equal-length segments articulated structure, without requiring central representation of the arm and definition of the medial joint location.

Supported by ISF and DARPA grants

Currents underlying the activity of CPG neurons B31/B32 in the buccal ganglia of Aplysia

Susswein A.J., Hurwitz I. and Koester J.

Faculty of Life Sciences, Bar Ilan University, Ramat Gan,

Israel and Center for Neurobiology and Behavior, Columbia

University, New York, NY

The B31/B32 neurons are key elements in a central pattern generator (CPG) in the Aplysia buccal ganglia. B31/B32 respond to excitation with a slow depolarization that, after a delay of 1-2 seconds, triggers a plateau depolarization reaching a value of $-20 \mathrm{mV}$. The plateau is maintained for $1-2 \mathrm{sec}$. Two electrode voltage clamping was used to characterize the currents underlying B31/B32 activity.

Two inactivating outward currents were found. Threshold for activating them was $-40 \mathrm{mV}$. At $0 \mathrm{mV}$ the currents activated rapidly, reaching a maximum current amplitude within 10-20 msec. The currents were differentially affected by TEA and 4-AP The 4-AP sensitive current reached $90 \%$ inactivation $\sim 320 \mathrm{msec}$ after the peak, and the TEA sensitive current reaching $90 \%$ inactivation in $\sim 2.7 \mathrm{sec}$. The rapidly-and slowlyinactivating currents had reversal potentials of $-70 \mathrm{mV}$ and $55 \mathrm{mV}$, respectively. From a holding potential of $-60 \mathrm{mV}$ (typical resting potential for B31/B32), the amplitudes of the rapidly- and slowly-inactivating currents at $0 \mathrm{mV}$ were $200 \mathrm{nA}$ and $160 \mathrm{nA}$, respectively. These currents probably contribute to the delay between a depolarizing stimulus and the plateau depolarization. 
A non-inactivating outward current was also found which was insensitive to TE $\AA$ or 4-AP. Its activation threshold was -20 $\mathrm{mV}$, and its amplitude at $0 \mathrm{mV}$ was $26 \mathrm{nA}$. It reversed at -35 $\mathrm{mV}$. This current probably functions during the plateau depolarization as a brake on the inward currents.

An inward current was observed during spontaneous buccal motor programs, and in response to the cholinergic agonist oxotremorine (Dembrow et al., 2002). Threshold for activation was $-50 \mathrm{mV}$. The current reached peak activation in $3.5 \mathrm{sec}$, and thereafter showed no inactivation. The reversal potential was close to $0 \mathrm{mV}$. The data may be sufficient to account for the intrinsic properties of the B31/B32 and its ability to initiate buccal motor programs.

The influence of heat acclimation on neuromodulation of thermoregulation and hypohydration by angiotensin II and nitric oxide

Schwimmer $\mathrm{H}^{1}{ }^{1}$, Gerstberger $\mathbf{R}^{2}{ }^{2}$ and Horowitz $\mathbf{M}^{1}$

${ }^{1}$ The Hebrew University of Jerusalem, Israel; ${ }^{2}$ Justus-Liebig University of Giessen. Germany

Acclimation to a new environment enhances the capacity and responsiveness of preexisting features, beneficial to the organism in its new environment. Coping with thermal stress depends on body fluid compartmentalization, altering with the course of heat acclimation. Converging input of thermal and osmotic stimuli in the MnPO nucleus controls the body temperature regulatory cascade. Based on the individual involvement of central Angiotensin II (AngII) and nitric oxide (NO) in thermoregulation and fluid balance, we aimed to elucidate the mutual effect of AngII and neuronal nitric oxide synthase (nNOS) in the integration of thermo- and osmoregulation during the course of heat acclimation. Rats were divided into euhydrated or hypohydrated $(-10 \%$ body weight.) groups of heat acclimated $\left(34^{\circ} \mathrm{C}\right)$, for 30 days (LTHA), 2days (STHA) and non-acclimated (C). 7-Nitroindazole (Ni) - nNOS blocker and AngII, separately or combined, were centrally administered. Three experimental paradigms were used: 1) in-vivo: measurements of vasodilatation and evaporative cooling thresholds, and heat endurance in conscious heat stressed $\left(39^{\circ} \mathrm{C}\right)$ rats. 2) Expression levels of AT1 and AT2 receptors and nNOS were measured in the hypothalamus (Western blot). 3) Real-time PCR was used to detect transcript levels of the coding genes. Results show a biphasic pattern of temperature thresholds for skin vasodilatation and salivation during the acclimation process. LTHA prolonged endurance, with a concomitant decrease in the AT1/AT2 receptor ratio in the cell membrane, and a drop in cytosolic AT2 receptors and nNOS levels. In hypohydration the AT1/AT2 receptor ratio decreased in $C$ and LTHA. Transcription also shows a biphasic profile during the course of acclimation. We conclude that heat acclimation produces a transition from AT1 to AT2 receptors, leading to a different signaling pathway of AngII, and changes in the mutual effects of NO and AngII on thermoregulation.

Apoptotic characteristics of cell death and the neuroprotective effect of carnosine-related compounds on pheochromocytoma PC12 cells exposed to Oxygen and Glucose Deprivation (QGD)

Tabakman $R$. , Jiang H. ${ }^{2}$, Levine R.A. ${ }^{2}$, Kohen . $^{3}$ and Lazarovici $\mathrm{P}$.

Dept. of Pharmacology and Experimental Therapeutics ${ }^{\prime}$ and Dept. of Pharmacy, School of Pharmacy, Hebrew University of Jerusalem, Jerusalem 91120 , Israel, ${ }^{2}$ William T. Gossett Neurology Laboratories, of Henry Ford Hospital, Detroit, $M I$ 48202, USA

The rat pheochromocytoma cell line $\mathrm{PC} 12$, which displays phenotypic characteristics of both adrenal chromaffin cells and sympathetic neurons is a useful system for exploring neuroprotective agents. Using a special device and undifferentiated PC12 cells, we established an in vitro model for ischemia by a combination of oxygen and glucose deprivation (1). The cell death induced in this ischemic mode was evaluated by a series of markers: lactate dehydrogenase (LDH) release, caspase 3 activation, the presence of cyclin D1, cytochrome $\mathrm{C}$ leakage from the mitochondria, $\mathrm{BAX}$ association with the mitochondria, cleavage of poly (ADP-ribose) polymerase (PARP) to an $85 \mathrm{kDa}$ apoptotic fragment, and DNA fragmentation. During the OGD insult, in the absence of reoxygenation, strong activation of the MAPK isoforms extracellular regulated kinase (ERK), c-Jun NH2-terminal kinase (JNK) and stress activated protein kinase (SAPK), also known as p-38 was measured. The detection of apoptotic markers and activation of MAPKs during the ischemic insult strongly suggest that apoptosis plays an important role in the $\mathrm{PC} / 2$ cell death.

Carnosine ( $\beta$-alanyl-L-histidine) and carnosine-related compounds (CRC) such as homocarnosine ( $\gamma$-aminobutyryl-L-histidine) are present in skeletal muscles
(1-20 $\mathrm{mM})$ and in the central nervous system (0.7-10 $\mathrm{mM})$ and may act as neuroprotective antioxidant compounds but their effect in $\mathrm{PC} 12$ ischemic model was not yet investigated Homocarnosine and carnosine provided a maximal neuroprotective effect of about $50 \%$ measured by the release of LDH and caspase 3 activities. Homocarnosine reduced OGD-activation of ERK 1 and ERK 2 by $40 \%$ and $46 \%$ respectively, and OGD-activation of JNK 1 and JNK 2 by $55 \%$ and $30 \%$, respectively. Therefore, neuroprotection conferrod by homocarnosine on PC12 cells exposed to ischemic insult may be explained by interference with the apoptotic process and/or related to the differential inhibition of MAPKs.

Tabakman R., Lazarovici P. and Kohen R. (2002) Neuroprotective effects of carnosine and homocarnosine on pheochromocytoma PC12 cells exposed to ischemia. $J$. Neurosci. Res. 68,463-469.

Nerve Growth Factor pretreatment attenuates

Oxygen-Glucose-Deprivation induced c-Jun NH2-terminal kinase 1 (JNK 1) and stress-activated kinases p-38 a and $p-38 b$ activation and confers neuroprotection in the PC12 model

Tabakman R. ${ }^{1}$, Jiang H. $^{2}$, Schaefer E. ${ }^{3}$, Levine R.A. ${ }^{2}$ and

Lazarovici $\mathbf{P}$

Dept. of Pharmacology and Experimental Therapeutics, School of Pharmacy, Faculty of Medicine, The Hebrew University of Jerusalem, Jerusalem

William T. Gossett Neurology Laboratories ${ }^{2}$, Henry Ford

Health System, Detroit, MI 48202, USA, Signal Transduction and Immunology, $Q C B^{3}-A$ Division of BioSource

International, 3 Avenue D, Hopkinton, MA 01748, USA.

We used an Oxygen-Glucose-Deprivation (OGD) model to investigate the neuroprotective effect of NGF. Pheochromocytoma PC12 neuronal cultures were exposed to OGD insult in a special device, followed by OGD-reperfusion under regular oxygen atmospheric conditions. Neuronal cell death induced in this model was measured by the release of lactate dehydrogenase (LDH) and the activity of caspase-3 enzyme.

Pretreatment of the cultures with $50 \mathrm{ng} / \mathrm{ml}$ of NGF, $18 \mathrm{~h}$ prior to OGD insult, conferred $30 \%$ of neuroprotection. The neuroprotective effect was similar to that obtained with cAMP-inducing neuropeptide Pituitary Adenylate Cyclase-Activating Polypeptide (PACAP); the antioxidant 4-hydroxy-2,2,6,6-tetramethylpiperidine-1-oxyl (Tempol) and the L-type calcium channel blocker nimodipine. However, treatment of the cultures with NGF concomitantly with the OGD insult, did not result in neuroprotection. These results may suggest that the neuroprotective effect of $\mathrm{NGF}$ involved protein synthesis.

Time course experiments showed marked activation of the ERK, JNK and p-38 MAPK isoforms during the OGD phase. Pretreatment of the cultures with $50 \mathrm{ng} / \mathrm{ml}$ of NGF $18 \mathrm{~h}$ prior to OGD insult, resulted in $50 \%$ inhibition of OGD-induced activation of JNK 1 , and $20 \%$ and $50 \%$ inhibition of OGD-induced activation of $p-38 \alpha$ and $\beta$, respectively. These findings support the notion that NGF confers neuroprotection from OGD-insult a phenomenon related to differential inhibition of MAPK stress kinase isoforms

PC 2 cells exposed to OGD offer an in vitro pharmacological model to investigate the molecular neuroprotective mechanisms of NGF.

M-current determines the firing pattern of $\mathrm{CA3}$ pyramidal cells

Tamir I. and Yaari Y.

Dept. of Physiology, Institute of Medical Sciences, Faculty of Medicine, The Hebrew University, Jerusalem 91120

The muscarinic-sensitive $\mathrm{K}^{+}$current (M-current; $I \mathrm{M}$ ) is a subthreshold noninactivating voltage-gated $\mathrm{K}^{+}$current that is generated in CNS neurons by heteromeric assemblies of $K C N Q 2,3$, and 5 gene products. It is widely accepted that $I_{\mathrm{M}}$ down-regulates intrinsic neuronal excitability, but its specific function in each type of neuron is still unknown.

We have investigated the role of $I_{M}$ in regulating the excitability of $\mathrm{CA} 3$ pyramidal cells (PCs), using rat hippocampal slices and standard current-clamp intracellular recording techniques. In vivo, these neurons generate either 'simple' (i.e. single) or 'complex' (i.e. burst of) spikes, depending on the behavioral state. In vitro, when injected with brief ( $4 \mathrm{~ms}$ ) depolarizing current pulses, all PCs fired only a single spike. Longer $(180 \mathrm{~ms})$ depolarizing stimuli evoked multiple spike responses, which accommodated rapidly and completely due to the activation of a slow afterhyperpolarization (AHP). Intrinsic bursts, comprised of tightly clustered spikes riding on a depolarizing envelope, were seen in $\sim 40 \%$ of the PCs, but only in response to high-intensity stimulation. 
Exposing the slices to the selective KCNQ channel blockers linopirdine or XE991 $(10 \mu \mathrm{M})$ markedly augmented the number of spikes evoked by long stimuli, and increased the propensity to burst-fire, even in response to threshold-straddling stimuli. Additionally, these blockers induced spontaneous, low-frequency oscillations of the resting potential that lead to rhythmic bursting. In contrast, exposing the slices to the KCNQ channel opener retigabine $(10 \mu \mathrm{M})$ reduced the number of spikes evoked at any stimulus intensity. We conclude that $I_{M}$ plays a decisive role in the discharge behavior of CA3 pyramidal neurons, limiting the number of spikes and the production of spike bursts. Given that $I_{M}$ is subject to down-modulation by many neurotransmitters, it_may be used by them to convert 'simple' to 'complex' spiking in different behavioral states.

Supported by BMBF, SFB 6006

Manipulating neuronal activity in culture overcomes the death signal induced by NGF low affinity receptor pathway Tayar S. Bogoch Y. and Linial M.

Dept. of Biological Sciences, Institute of Life sciences, The

Hebrew University, Jerusalem

Understanding the effects of NGF activation of low affinity NGF receptor $p 75^{\mathrm{NRR}}$ and its relationship with depolarization is likely to shed light upon the much disputed effects of this receptor on survival of neurons. We have hypothesized that synaptic depolarization would modulate concomitant neurotrophin receptor signaling, thereby integrating the activity state of a synapse with the state of neurotrophic signaling available at the synapse. P19 embryonal carcinoma cells that serve as a model for studying differentiation processes including commitment to cell lineage, were used following activation of neuronal differentiation. The potential of these cells to mature and efficiently release neurotransmitter (NT) was established in our lab. We discovered that several variables, most notably, cell density and various neurotrophic factors affects neuronal maturation, survival and the choice of NT phenotype. P19 cells express a large quantity of p $75^{N T}$ the low affinity NGF receptor, on the plasma membrane, but not the high affinity receptor. TrkA allowing the study of p $75^{\mathrm{NTR}}$ without the influence of TrkA activation. No difference was monitored in the expression level of TrkA when najve, untreated cells were compared to NGF treated ones. Immediately following their plating and adhesion, cells were supplemented with increasing amounts of NGF $(0-200 \mathrm{ng} / \mathrm{ml})$, as well as varying potassium levels $(0-30 \mathrm{mM})$ and survival was monitored by probes indicative for membrane integrity and membrane potential. Increasing the level of exposure to NGF caused a switch of a survival signal to a death signal. Furthermore, we showed that low level tonic depolarization that mimics the physiological state of activation, reversed the death signal induced by the NGF. We suggest that P19 via its p75 $5^{\mathrm{NTR}}$ activation is a suitable system to control the delicate balance between apoptotic death, early differentiation and neuronal activity. We conclude that modulation of neurotrophin-induced signaling via changes in synaptic activity may be an important paradigm for the development of novel therapeutic strategies aumed at inducing, enhancing, and accelerating neural growth and survival.

Object recognition test: A tool for evaluation of cognitive impairment and therapeutic opportunities after traumatic

brain injury ${ }_{\text {Tsenter } \mathrm{I}^{-}, \text {Beni SM. }}{ }^{1}$, Yatsiv I. ${ }^{2}$., Biegon A. ${ }^{3}$ and Shohami E. ${ }^{1}$ Pharmacology, Hebrew University, Jerusalem, Israel: ${ }^{2}$ Physical Rehabilitation, Pediatric Intensive Care. Hebrew University and Hadassah, Jerusalem, Israel; ${ }^{3}$ LBNL, Berkeley CA, USA

Introduction: Cognitive deficits are common after brain injury. We have shown hippocampal dysfunction in a mouse model of closed head injury (CHI). Erythropoietin (EPO), a kidney-derived cytokine with growth factor and anti-apoptotic properties, is present in the brain and elevated after oxidative stress (OS). We recently showed that Epo is neuroprotective after CHI. OS is implicated in hippocampal formation of memory, while overexpressoin of SOD1 is associated with impairment of spatial learning and disability to express LTP, an NMDA-dependent process. NMDA receptors are down-regulated 1-7d after $\mathrm{CHI}$. This study was designed to evaluate the effect of Epo, NMDA and SOD on cognitive function of mice after CHI. Methods: Prior to $\mathrm{CHI}$ and 3-14 thereafter, object recognition test was performed to evaluate cognitive function. Mice were placed in a cage with two identical objects and the time spent exploring each object was recorded for $5 \mathrm{~min}$. Four or $24 \mathrm{~h}$ later, one object was replaced and exploration time was recorded Differences between times spent at a familiar and a new object were used to evaluate effect of treatments on cognitive ability of CHI mice. HuEPO $(5,000$ units $/ \mathrm{kg} /$ dose $)$ was injected 1 and $24 \mathrm{~h}$ after CHI.
NMDA $(20 \mathrm{mg} / \mathrm{kg}$ ) was injected 24 and $48 \mathrm{~h}$ after CHI. The effect of SOD was studied in SODI-overexpressing mice Results and Discussion: Normal mice spent longer time exploring a novel object. CHI mice did not differentiate between the objects within Iwk after injury but this ability was later regained. Epo and NMDA treated mice displayed significantly longer exploration time of the novel object compared to the familiar one $(p<0.01)$, hence their behavioral pattern resembled that of normal mice with intact memory Loss of recognition-memory was not prevented by SOD 1 overexpression. We propose that the object recognition test clearly demonstrates cognitive impairment after $\mathrm{CHI}$, and is sensitive to various therapeutic strategies, acting via different mechanisms.

\section{Apathy, depression and motor symptoms in Parkinson's
disease}

Tsitrinbaum $\mathrm{Z}^{1,2}$, Tomer $\mathrm{R}^{1,2}$ and Aharon-Peretz J. ${ }^{1}$

${ }^{7}$ Cognitive Neurology Unit, Rambam Medical Center, Haifa

${ }^{2}$ Dept. of Psychology \& Brain and Behavior Center, University of Haifa

Although apathy is traditionally considered an aspect of depression, it has been proposed to exist also as a separate behavioural syndrome, most likely reflecting dopaminergic dysfunction. Apathy without depression has been found in $12 \%$ of PD patients. However, it is not clear why some PD patients display only apathetic symptoms whereas others show either depression alone, or both. The current study examined the association between depression, apathy, and clinical characteristics of PD, which are known to reflect the degree of dopaminergic depletion, in non-demented patients with idiopathic PD. Method: Forty two non-demented patients with idiopathic PD were recruited: Twenty three with right side onset of the disease, nineteen subjects with left side of onset of the disease. Thirty age-, sex- and education-matched healthy volunteers served as controls. Motor symptoms of the PD subjects were assessed using the UPDRS. All subjects completed self-report questionnaires measuring apathy (Apathy Evaluation Scale) and depression (Beck Depression Inventory). Results: PD patients were significantly more depressed and apathetic than the control subjects whereas they did not differ in terms of demographic features. Although apathy and depression were significantly correlated in PD patients, several dissociations were found, suggesting that dysphoria in PD may be associated with mesolimbocortical dopaminergic depletion, whereas apathy may be related to both mesolimbocortical and nigrostriatal dopaminergic deficit in the disease.

Juvenile stress impairs adulthood avoidance learning in Juvenile stress impairs aduit potential role of $\mathrm{GABA}$

Tsoory M. M. and Richter-Levin G.

Dept. of Psychology. Faculty of Social Sciences, University of Haifa

Epidemiological studies indicate that children exposed to early adverse experiences are at increased risk for the development of affective disorders, particularly depression and anxiety disorders. Several lines of evidence suggest the involvement of a GABA deficit in those disorders. Furthermore, pharmacologically increasing GABAergic neurotransmission was found to exert antidepressant effects and to reduce anxiety.

In order to investigate how early adverse experiences relate to GABAergic dysfunction and impaired cognitive and emotional abilities in adulthood, rats underwent stressful experiences on 27-29 PNDs and at 56 PNDs their exploratory behavior and avoidance learning were assessed. At 57 PNDs limbic system tissue was collected for WB analysis of GABA receptor subunits.

Adult rats (56 PNDs) exposed to stress at 27-29 PNDs did not engage in exploratory behavior in a novel setting and exhibited poor avoidance learning compared with control. In the two way shuttle avoidance task two typical responses were evident among rats exposed to stress at 27-29 PNDs, about 2/3 learnt well the escape response but not avoidance, while the remaining $1 / 3$ learnt neither, thus exhibiting behaviors reminiscing of PTSD and depressed patients respectively.

WB analysis is currently conducted in order to examine whether these cognitive and emotional deficits are associated with abnormal structure and function of the GABAergic system in the limbic system.

The assessment of the "GABAergic Hypothesis" may have the potential to contribute significantly to the understanding of the neurophysiology of affective disorders and may serve to direct thinking about potential novel courses for drug development. 
Is the 'Error-Related Negativity (ERN)' specific to error making?

Tzur G. and Berger A

Ben Gurion University of the Negev

The anterior cingulate cortex (ACC) is considered to be involved in executive control of actions, such as monitoring conflicting response demands, detecting errors, and evaluating the emotioinal significance of events. Electro-physiological studies have shown about $100 \mathrm{~ms}$ after subjects made an error, a focal negative wave in the electrical activity over the medial frontal cortex. Source localization indicates the ACC as the plausible source of this negative wave. this wave has been attributed to the subject's awareness of making an error and is called an Error-Related-Negativity (ERN), since it has a negative amplitude peak and is related to error-making. The present study tests the plausibility of obtaining a similar negative wave when a subject just perceives an erroneous situation. Our preliminary results support this hypothesis.

Inactivation of T-type Ca2+ channels: A mechanism underlying short-term plasticity of intrinsic membrane

properties ${ }_{\text {Uebachs } M .}{ }^{1}$, Perez-Reyes E. ${ }^{2}$ and Beck $H^{1}{ }^{1}$

Dept. of Epileptology, University of Bonn, Germany;. ${ }^{2}$ Dept. of Pharmacology, University of Virginia, USA

T-type $\mathrm{Ca}^{2-}$ currents possesses a characteristic voltage-dependence, apart from other types of $\mathrm{Ca}^{2+}$ channels, including a low threshold of activation, and a slow deactivation time course. Furthermore, the steady-state inactivation curve possesses a midpoint close to the membrane potential of many neuronal cells, causing a steep dependence of channel availability on membrane potential. Following depolarizations, channel availability depends on the recovery rate from inactivation.

We have svstematically examined the recovery from inactivation of the three pore-forming $\mathrm{Ca}^{2+}$ channel subunits $\left(\mathrm{Ca}_{\mathrm{v}} 3.1, \mathrm{Ca}_{\mathrm{v}} 3.2, \mathrm{Ca}_{\mathrm{v}} 3.3\right)$ underlying T-type $\mathrm{Ca}^{2+}$ channels, expressed in HEK cells. We found that the recovery time course following conditioning prepulses to -20 or $-50 \mathrm{mV}$ was well fit by a biexponential equation. Increasing the duration of the conditioning prepulse (from 0.1 to $300 \mathrm{~s}$ ) caused a marked slowing in recovery rates in all three subunits, with complementary but overlapping ranges of recovery rates $\left(\mathrm{t}_{\text {slow }}\right.$ : $0.18-1.57 \mathrm{~s}, 2.01-7.33 \mathrm{~s}, 0.18-3.80 \mathrm{~s}$ for the three subtypes respectively). Varving the voltage of the conditioning pulse had no effect on the recovery rates for any subunit.

In marked contrast to continuous depolarizations, trains of mock action potentials at increasing frequencies $(1-100 \mathrm{~Hz})$ caused increasing inactivation of $\mathrm{Ca}^{2}$ currents, but no concomitant slowing of recovery rates. Likewise, varying the duration of action potential trains ( $30 \mathrm{~Hz}, 0.1-300$ seconds) did not affect the recovery rates. Similar findings were obtained in native thalamic relay neurons, dissociated enzymatically from rat brain.

In summary, pronounced scaling of recovery rates is invoked by continuous subthreshold depolarizations, but not by series of action potentials. This property of $\mathrm{T}$-type $\mathrm{Ca}^{2+}$ channel pore-forming subunits causes them to retain a trace of subthreshold membrane potential variations, but not action potential firing rates, encoded via scaling of recovery rates.

Bias in the measurement of auditory response curves Ulanorsky N., Las-Ahdut L. and Nelken I.

Dept of Physiology and the Interdisciplinary Center for Neural Computation. Hebrew University

Several functional roles have been suggested for neuronal adaptation, including gain control, improvement of stimulus discriminability and optimization of information transmission. We have previously shown that neurons in primary auditory cortex (A I) respond more strongly to a sound when it appears rarely in a sequence than to the same sound when common, as a result of stimulus-specific adaptation - suggesting a role for adaptation in novelty detection. Here we propose a new role for adaptation: enhancement of responses to eccentric stimuli. Adaptation can alter neuronal tuning curves, but it is widelyassumed that adaptation-induced bias is minimized when stimuli are randomized, equi-probable and have the same amplitude. We therefore checked the effect of adaptation on measurement of tuning curves. We recorded activity of neurons in Al of halothane-anesthetized cals, in response to randomly presented tones with 10 repetitions of 20 frequencies, covering a range of 1 octave. We found that adaptation was stronger for frequencies at the center of the frequency range, than for eccentric frequencies at the edges. This occured since the trials that preceded 'central' frequencies had a smaller parametric difference from those frequecnies (average difference of $1 / 4$ octave) then the trials that preceded eccentric frequencies (average difference of $1 / 2$ octave). Since adaptation is stronger for similar consecutive stimuli, this resulted in stronger adaptation to 'central' frequencies, and in enhanced responses to eccentric stimuli. This finding has both methodological and functional consequences. Methodologically, measuring response curves using unbiased stimuli (randomized and equi-probable), nevertheless results in a bias in the shape of the response curves, with selective suppression at 'central' stimuli. Functionally, the enhancement of responses to eccentric stimuli may facilitate their detection, which, as suggested by some models, may aid in frequency discrimination.

\section{Characterization and function of TRKA mutations in} Israeli patients with congenital insensitivity to pain with anhidrosis (CIPA)-peripheral neuropathy,

Umansky-Safanova $\mathrm{S}^{1}{ }^{,}$, iang $\mathrm{H}^{2}$, Galski $\mathrm{H}^{3}{ }^{3}$, Blumenfeld A. ${ }^{4}$, Oddoux $\mathrm{C}^{5}$ Axelrod $\mathrm{F}^{5}$ and Lazarovici $\mathrm{P}$.

Dept of Pharmacology, School of Pharmacy, Hebrew University of Jerusalem ; ${ }^{2}$ Laboratory of Molecular Immunology. Sheba Medical Center. Tel Hashomer.

${ }^{3}$ Molecular Biology, Hadassah University Hospital, Jerusalem. Molecular Biology, Hadassah University Hospital, Jerusal Hospital, Detroit, MI; 'Human Genetics, NYU Medical Center. New York, NY, USA

Congenital Insensitivity to Pain with Anhidrosis (CIPA) is an autosomal recessive hereditary sensory neuropathy characterized by loss of neurons from the peripheral and autonomic (sympathetic) nervous systems, associated with lack of sweating (anhidrosis) and insensitivity to pain. Nerve Growth Factor (NGF) regulates the development and survival of nociceptive and thermoceptive sensory neurons through its high affinity, trkA receptor. The human TRKA gene (NTRK1), located on chromosome 1q21-q22 encodes the receptor tyrosine kinase for NGF. Several lines of evidence indicate that mutations/polymorphism in the trkA gene result in CIPA Most of the reported patients reside in the Middle East/Mediterranean region.

In the present study we characterized the trkA mutations in the Israeli patients, in order to identify structural and physiological impairment(s) in the trkA gene and its product, and to correlate it with clinical symptoms and severity. Five novel mutations have been detected on exons 15-17, the tyrosine kinase domain located in the carboxy terminal of the trkA receptor gene. Among them mutations Arg761Trp is the most typical of Israeli CIPA patients. Mutations related to CIPA were performed by site-directed mutagenesis in order to investigate structure-function relationship of trkA receptor. This trkA receptor structure-function study is intended towards understanding the functions of the intracellular catalytic domain, and other domains of trkA in the etiology of CIPA, and possibly of other neurological trkA related disorders. The long term outcome of this study is aimed towards the development of genetic approaches for CIPA patients.

Supported by a grant from the United States-Israel Binational Science Foundation (BSF), Jerusalem, Israel.

\section{Characterization of local brain circuits participating in} memory consolidation of novel taste learning

Vardinon-Friedman $\mathrm{H}$. and Rosenblum $\mathrm{K}$.

Center for Brain and Behavior, Haifa University, Haifa 30905

Long term memory is the mental faculty by which experiences cause a persistent modification of cognition or behavior. The experience is encoded through neuronal activity leading to long term changes that are mediated by protein phosphorylation and synthesis.

In this research project we focus on the identification and characterization of the cortical circuit employed for consolidation of novel taste learning Studies based on pharmacological, electrophysiological, and lesion approaches have demonstrated that specific brain regions take part in the normal encoding of taste memories. Of these, the insular cortex was proved to be essential for taste memory consolidation. However, the identity of a neuronal circuit sub serving a given taste memory consolidation is unclear.

In order to identify the taste circuit in the gustatory cortex and to trace the changes in it, we fluorescently label specific cellular proteins that were proved to be essential for memory consolidation process (immediate early genes, kinase activity), along with cytoskeletal, and synaptic markers. Imaging of these selected molecules, using retrospective immunofluorescence, cnables focused observation on specific cellular populations, and yiclds information about neuronal activity together with sub cellular localization, at a certain time point.

It is important to define how the elementary bricks of cortical microcircuits are interconnected, as functional signatures of cortical neurons are determined by their intra-acrial circuitry. Moreover, this data is likely to be instrumental in determining circuit-level neuronal function throughout the cortex 
PolyADP-ribosylation by activated ERK1/2: A ovel nuclear mechanism apparently involved in memory formation Visochek L. ', Klein R. , Yao Z. ${ }^{2}$, Seger R. ${ }^{2}$ and Cohen-Armon $\mathrm{M}^{1}$ "Sackler School of Medicine, Tel-Aviv University. Tel-Aviv; 'Biological Regulation, Weizmann Institute of Science. Rehovot

Our findings unveil a novel signal transduction mechanism: Activated ERK $1 / 2$ modifies chromatin bound proteins by polyADP-ribosylation. PolyADP-ribosylation is a post-translational modification of proteins catalyzed mainly by the abundant and highly conserved nuclear protein PARP-1. This reaction is initiated by ADP-ribosylation on glutamic and aspartic moieties and proceeds by a rapid and transient $\left(\mathrm{t}_{1 / 2}=1\right.$ min) polymerization of the ADP-riboses into high polymers. PolyADP-ribosylation of chromatin bound proteins perturbs their binding to DNA, and thereby influences their activity and modifies the chromatin structure. This modification affects DNA replication, transcription and repair. PARP-1 is activated by its binding to free DNA-endings in nicked DNA, being one of the known proteins expressing DNA-damage scanning activity. However, recently we found an alternative mode of PARP-1 activation in the absence of DNA damage: In brain cortical neurons PARP-1 was rapidly activated downstream to phospholipase-C activation in response to high frequency depolarizing stimulation, or in response to stimulation by neurotrophins or serotonin. Furthermore, here we present for the first time evidence for an instantaneous activation of PARP-1 by activated ERK1/2 in the cell nucleus. This process is also active in nuclear proteins extract. The rapid modification of chromatin bound proteins by polyADP-ribosylation downstream to ERK-phosphorylation cascades has a wide spectrum of implications. One of these implications refers to the formation of long-term memory. Cohen-Armon and collaborators have recently demonstrated in the marine mollusk Aplysia the essential role of polyADP-ribosylation in LTF, learning and long-term memory.

Eye dominance reflected by unequal interocular stability of eye movements during fixation.

Wagner M. and Ehrenstein W.H.

Smith Family Lab. for Collaborative Research in

Psychobiology. Hebrew U. Jerusalem; The College of Judea \& Samaria, Ariel Israel: Leibniz Research Center for Working

Environment \& Human Factors, U. of Dortmund, Germany

We investigated the relation between eye dominance and binocular eye movements during fixation under unrestrained head posture. A fixation mark was always presented at the center of the screen, accompanied by two lines, that were either vertical parallel (2D representation), or tilted in a convergent or divergent way to create a pictorial depth cue $\left(2 \frac{1}{2} \mathrm{D}\right)$, so that the cross appeared either in a near or far perspective.

Eye dominance was determined by a questionnaire (Coren 1993) and a sighting task, which required binocular and monocular alignment of a target through a hole in the middie of a card. The eye that is co-linear with the hole and the target is revealed as the dominant eye (card test). Eye movements were recorded (EyeLink system).

Seven subjects were examined (two with left-eye, and one with no dominance). The dominant eye was significantly more accurate and stable in keeping fixation than the non-dominant. This difference between eyes increased when the display was changed from $2 \mathrm{D}$ to $2 \frac{1}{2} \mathrm{D}$ representation.

Our data show that fixational eye movements actually reflect eye dominance which determines the direction associated with visual straight ahead (egocentric localization), possibly to avoid diplopia in stereo depth, but already effective with pictorial depth cues.

Sipported by the Israel Science Foundation (ISF) Grant $\# 8009$.

\section{Gene and protein expression profiles of neuroprotective} and antiapoptotic action of Rasagiline in $\mathrm{PC} 12$ cell culture Weinreb O., Bar-Am O., Chillag-Talmor O., Amit T. and Youdim MBH.

Eve Topf \& US National Parkinson's Foundation Centers of Excellence for Neurodegenerative diseases, Rappaport Faculty of Medicine, Technion, Haifo

Rasagiline, a potent novel anti-Parkinsonian drug, exerts a neuroprotective activity and prevents the fall in mitochondria membrane potential and the opening of mitochondrial voltage-dependent anion channel in cultured neuronal cells and in animal models of head trauma and MPTP induced neurotoxicity. To verify whether rasagiline affects the suppression of the cell death process via mitochondrial signal transduction pathway, we examined in this study the mechanism underlying neuroprotection by this drug in serum deprivation model of rat pheochromocytoma (PC-12) cells. We have demonstrated a concentration-dependent correlation of rasagiline between modulation of cell survival and the related gene pathways involved in neuroprotection. $\mathrm{PCl} 2$ cells viability was significantly induced $(-30 \%)$ by rasagiline $(1$ and $10 \mathrm{mM})$ after $24 \mathrm{hr}$ of serum deprivation, either measured by an apoptotic cell death detection ELISA kit or by MTT reduction assay. The involvement of Bcl-2 family members, which participate in the regulation of apoptosis, was studied using quantitative real-time RT-PCR technique. Rasagiline, dose dependently $(0.1-10 \mathrm{mM})$ significantly upregulated mitochondrial antiapoptotic Bcl-2 family genes, $\mathrm{Bcl}-\mathrm{X}_{\mathrm{L}}$ and $\mathrm{Bcl}-\mathrm{w}$ and downregulated proapoptotic mRNAs of Bad and Bax at 3 and $24 \mathrm{hr}$. Western analysis of these proteins confirmed the gene changes. Our results exhibit doseand time- dependent activity of rasagiline, induced cyto-protective genes and protein expression that may rescue neurons from apoptosis mediated by the mitochondrial death cascade and protect declining neurons in neurodegenerative diseases.

\section{IL-1-signaling plays an important role in the development} of opiate tolerance

Wolf G., Yirmiya R., Bar-Hen H., Goshen I. and Shavit Y Dept. of Psychology, Hebrew University, Mount Scopus,

The development of tolerance is a prominent characteristic of chronic opiates, which has immense clinical implications. In recent years, the involvement of proinflammatory cytokines in the development of acute and chronic opiate tolerance has been suggested. The cytokine interleukin-1 (IL-1) is known to modulate pain perception in various inflammatory conditions, under which it primarily induces hyperalgesia. Recently, we have demonstrated that IL-1signaling is also involved in determining baseline pain sensitivity, and plays an anti-analgesic role in acute opiate analgesia. The present study examined whether IL-1 influences the development of morphine tolerance. Mice were injected with morphine twice a day $(10 \mathrm{mg} / \mathrm{kg}$, sc, at $8 \mathrm{am}$ and $4 \mathrm{pm})$ for five consecutive days. Morphine analgesia was assessed using the hot-plate latency, measured one hour following each morning injection. Subjects were 2 strains of mice genetically impaired in IL-1 signaling (deletion of IL-1 receptor type I, or transgenic over-expression of IL-1 receptor antagonist (IL-lra)) and their wild-type (WT) controls. Other WT mice were chronically treated with IL-1ra or placebo, using implanted micropumps, and were also tested for tolerance development. The two mutant strains displayed significant morphine analgesia throughout the 5 morphine days, while their WT controls developed morphine tolerance within a couple of days. Similarly, mice treated chronically with IL-l ra displayed significant morphine analgesia throughout the 5 morphine days, while placebo-treated mice developed morphine tolerance within a couple of days. These results suggest that IL-1 signaling plays an important role in the development of morphine tolerance.

\section{Functional role of neuronal $\mathrm{KCNQ} / \mathrm{M}$ channels} Yaari Y. and Yue $\mathrm{C}$

Dept. of Physiology, Institute of Medical Sciences, Hebrew University-Hadassah Faculty of Medicine, Jerusalem 91120

$\mathrm{KCNQ} 2 / 3 / 5$ channel subunits are widely expressed in peripheral and central neurons, where they give rise to a muscarinic-sensitive, subthreshold and noninactivating $\mathrm{K}^{+}$ current $\left(I_{M}\right)$. Activation of $I_{M}$ may contribute to spike frequency adaptation during sustained depolarizations, but is considered too slow to influence the repolarization of solitary spikes. In hippocampal pyramidal neurons, however, reducing $I_{\mathrm{M}}$ with selective KCNQ channel blockers markedly enhanced the spike afterdepolarization (ADP), thereby converting solitary spikes to high-frequency bursts of 3-7 spikes ('complex' spikes). Conversely, increasing $I_{M}$ with a KCNQ channel agonist reduced the spike ADP and induced regular firing in bursting neurons. By effectively controlling the spike ADP waveform, KCNQ channels play a critical role in determining the intrinsic firing patterns, and hence, the input-output relations of neurons.

\section{Salt appetite is not increased in swimmers}

Yativ M., Stern S. and Leshem M.

Psychology Dept. and Brain \& Behavior Center, University of Haifa

Varied forms of exercise increase salt and sweet preference. Sweet preference is related to energy replenishment. Salt preference might be due to the sympathetic arousal of exertion. but it has also been reported that it is related to the amount of sweat loss, in turn suggesting that it is sodium loss in sweat that might cause the increased preference. As a further test of this possibility, we examined salt preference after swimming training, where exertion is similar to other forms of exercise, 
but sweating is reduced. If salt preference is indeed related to sodium loss in sweat, we would expect a minimal increase in salt preference after swimming. Twenty youths $(14.4 \pm 1.2 \mathrm{y})$ training for the Haifa Maccabi swimming team participated. Salt and sweet preference were measured before and after a 60 min training session, and level of exertion and sweat loss were monitored. No increase in salt preference was found in 3 tests of salt preference, while preference for concentrated sweet solution did increase. In comparison to other studies, sweat loss and post-exercise salt preference were lower. These results are a further indication that sodium loss in sweat may be the cause of increased salt appetite after exercise, and add weight to the proposal that salt preference in humans may be increased by physiological sodium need, as it is in animals. Supported by Israel Science Foundation Grant 902/00-2 to Micah Leshem

LPS-induced heat shock protein response in mouse brain and human astrocytoma cells

Yavelsky V. ${ }^{1}$, Wolfson M. ${ }^{1}$, Vais O., Sidorik L. ${ }^{2}$ and Fraifeld

$\mathrm{V}$

IBen-Gurion University of the Negev, Beer-Sheva, Israel;

${ }^{2}$ Institute of Molecular Biology, Kiev, Ukraine.

Lipopolysaccharide (LPS) is a classic trigger of acute phase response (APR), which manifests in a non-specific activation of the immune system and in thermoregulatory changes. The brain is one of the LPS targets. Heat shock protein response (HSPR) represents another non-specific protective mechanism. which is challenged by different and generally strong stressful stimuli. Both of these responses are altered with age. Although APR and HSPR are generally considered two independent systems induced by stimuli of different natures and strengths, there is evidence that they may modulate or interact with each other. Whether or not LPS affects the level of CNS HSPs, has hardly been addressed. The present study was undertaken to examine this issue. Male CD-1 mice aged 2 weeks to 12 months old were injected with LPS $(1 \mathrm{mg} / \mathrm{kg}$, ip) and at different times post-injection their brains were removed for preparation of tissue extracts. Western blot analysis revealed that the brain HSPs are differentially altered with age and in response to LPS. Basal level of Hsp90 peaks at 2 months and decreases in middle-age mice, while Hsp60 and Hsp27 appear unaltered within the tested age range. Treatment with LPS results in clear Hsp27 and Hsp90 up-regulation in young micc ( 2 months old), without apparent changes in premature mice and a lack of, or attenuated response in middle-age group. Supplementation of LPS to human U251 astrocytoma cell cultures leads to a clear increase of $\mathrm{Hsp} 90$, slight elevation in $\mathrm{Hsp} 27$, and insignificant changes in Hsp60. Analysis of in vivo and in vitro kinetics points to a relatively rapid HSPR to LPS, suggesting that an increased intensity of the bands could mainly be attributed to post-transcriptional alterations rather than to activation of the HSP gene expression

Supported by a grant from the United States-Israel Binational Science Foundation (BSF), Jerusalem. Israel.

Simulating octopus arm movements to study motor control Yekutieli Y. ., Hochner B. ${ }^{1}$ and Flash T.

${ }^{I}$ Neurobiology and Interdisciplinary Center for Neural Computation, Hebrew University: ${ }^{2}$ Computer Science and Applied Mathematics, Weizmann Instituteof Science

Octopus arms are highly flexible, move with a wide range of velocities, are able to grip objects and exert great force and can delicately manipulate objects. Octopus arms belong to a group of organs termed muscular hydrostats, which are built almost entirely of muscles with no rigid skeletal support. The basic biomechanical principles that govern the movements of muscular hydrostats are relatively well understood. However, how this hyper-redundant arm with its virtually infinite number of degrees of freedom is controlled, is largely unknown.

Here we describe a dynamic model of the octopus arm that is used to study possible control strategies of reaching movements where a bend in the arm travels towards the tip.

The model includes both external forces (gravity, buoyancy and water drag forces) and internal forces (muscle forces and constant volume constraint forces). It is controlled by an activation signal (a simplified neuronal command) that travels along the arm.

The main findings are:

1. A simple activation wave, which moves at a constant speed, was sufficient for the model to replicate the natural reaching movement with similar kinematic characteristics.

2. The biomechanical mechanism that produced the reaching movement was a stiffening wave a wave of muscle contraction that pushes forward a bend along the arm.

3. We investigated the possibility that the control of only two parameters might fully specify the extension movement: the amplitude of the activation pulse (that leads to the generation of muscle force) and the activation time (the time it takes the activation wave to travel along the arm). We showed that the same kinematics can be achieved by applying different activation amplitudes above some threshold. Large amplitudes generate larger forces that increase the arms stability against perturbations without changing the kinematic characteristics of the movement.

Oxidative stress leads to changes in the cellular redox state which correlates with cell death in in-vitro model of mechanical stretch injury

Yeshurun-Cohen A., ${ }^{1,}$, Kohen $\mathrm{R}^{2}$, and Shohami E. ${ }^{1}$ Depts. of ${ }^{l}$ Pharmacology and ${ }^{2}$ Pharmaceutics, Hebrew University, School of Pharmacy, Jerusalem

Background: Astrocytes are the most abundant non-neuronal cells in the brain. They provide a supportive environment to the neurons by exchanging products which are essential to the normal neuronal function and protect neurons against toxic mediators and hostile environment. Glutathione is one of the products which is being exchanged between astrocytes and neurons. It is sensitive to oxidative stress and protects the brain directly and indirectly from reactive oxygen species (ROS) which are massively produced in trauma, ischemia or neurodegenerative processes. Objective: To evaluate changes in levels of reduced (GSH) and oxidized (GSSG) glutathione in response to stretch injury of different severities in astrocytes and epithelial cell line, in correlation with cell death Methods: Primary astrocytes cultured from newborn mice and $\mathrm{HaCaT}$, transformed epithelial cell line, were grown in wells on a flexible silastic bottom. A cell injury controller was used to produce a rapid pressure of known amplitude and duration leading to a stretch injury, which deforms the cells and serves as our model system. Cells were collected 1 hour after the trauma, denaturated and injected into HPLC-ECD. Cell survival was measured by MTT method. Results and discussion: The survival of both cell types decreased with increased degree of trauma. In parallel, a decrease in GSH as compared with control was found in both cell types and elevation in GSSG was noted in the astrocytes. Thus a total decrease in the ratio GSH/GSSG occurred as a result of cell injury, and the redox potential calculated by the Nernst equation was found to be less negative in severe trauma as compared to control. Our results indicate that mechanical injury leads to changes in the redox state reflecting oxidative stress, which in turn correlates with cell death. Thus, the ratio GSH/GSSG can serve as a sensitive cellular marker for oxidative stress after injury, and as a tool to evaluate novel antioxidant therapies.

Tomosyn regulates the priming step in a

calcium-dependent manner

Yizhar O. ${ }^{1}$, Matti U. ${ }^{2}$, Melamed R. ${ }^{1}$, Hagallili Y. ${ }^{1}$, Bruns D. ${ }^{2}$, Rettig J.2, and Ashery U.

${ }^{I}$ Dep. Neurobiochemistry, Tel Aviv University, ${ }^{2}$ Physiologisches Institut, Universititt des Saarlandes, Germany.

The formation of a heterotrimeric SNARE complex is a crucial step in the pathway leading to the fusion of neurotransmitter-containing vesicles. The availability of SNARE proteins to form SNARE complexes is limited by various protein-protein interactions. One such interaction, between Syntaxin and Munc18, prevents Syntaxin from forming SNARE complexes. m-Tomosyn is a brain specific, $130 \mathrm{kDa}$ protein that is capable of disrupting the high-affinity interaction of Syntaxin with Munc18. Tomosyn binds to Syntaxin and forms a novel 10S complex with Syntaxin-1, SNAP-25, and synaptotagmin. However, the precise function of tomosyn is not known. Using morphological, capacitance and amperometric measurements, we investigated the effect of tomosyn on the different kinetic components of exocytosis in adrenal chromaffin cells. Overexpression of tomosyn reduces the release probability and causes a decrease in the number of fusion competent vesicles. The number of docked vesicles remains unchanged, indicating that tomosyn inhibits the priming step. Interestingly, during prolonged stimulation with elevated $\left[\mathrm{Ca}^{2+}\right]_{\mathrm{i}}$ this inhibition is partially removed. Calcium-ramp experiments indicated that tomosyn raises the requirement for $\mathrm{Ca}^{2+}$ in exocytosis without changing the calcium cooperativity. Overexpression of a tomosyn mutant lacking the C-terminal Synaptobrevin-homology domain caused a similar reduction in secretion and shift in calcium-dependence under steady-state conditions. However, these changes were abolished upon elevation of $\left[\mathrm{Ca}^{2+}\right]_{i}$. These results suggest that tomosyn specifically inhibits the priming step and impairs, partially through its Synaptobrevin-homology domain, the efficiency of vesicle pool refilling in a calcium-dependent manner. 
Involvement of Bcl-2 and Bax in neuroprotection by lithium against MPTP neurotoxicity

Youdim MBH. and Arraf $Z$.

Eve Topf and US National Parkinson 's Foundation Centers of Excellence for Neurodegenerative diseases and Depts. of Pharmacology, Technion, Haifa

Lithium has been reported to exert neuroprotective activity in cell several neuronal cell culture models against glutamate toxicity. Since lithium has been shown to activate the antiapoptotic Bcl-2 family protein we have examined its effect on the ability of the parkinsonism neurotoxin, MPTP (N-methyl-4-phenyl-1,2,3,6-tetrahydropyridine) to deplete striatal dopamine in mice. Mice were fed for 5 weeks with diet containing $1.1,2.2,3.3$, and $4.4 \mathrm{~g} / \mathrm{kg}$ lithium chloride ( $\mathrm{LiCl}$ ) for 4 weeks, during which serum levels of lithium were monitored. The $3.3 \mathrm{~g} / \mathrm{kg}$ lithium diet gave serum level value very similar to what is observed in lithium therapy and the $4.4 \mathrm{~g} / \mathrm{kg}$ well above this. At the end of this period the mice received $24 \mathrm{mg} / \mathrm{kg}$ MPTP i.p. once daily for three days and sacrifised 4 days later. A direct relation was established with the increase in serum lithium and its ability to prevent MPTP induced depletion of striatal dopamine (DA), DOPAC and HVA. With the highest lithium diet there was an almost complete prevention of striatal dopamine depletion and the reduction in tyrosine hydroxylase activity and protein. Lithium prevented the increase in dopamine turn over (DOPAC +HVA/DA) normally observed in MPTP treatment Lithium did not interfere with the metabolism of MPTP nor its brain uptake since the level of its monoamine oxidase (MAO) B derived metabolite, $\mathrm{MPP}^{+}$in striatum was almost identical in lithium and non-lithium treated mice. Striatal Bcl-2 was significantly decreased, while Bax was increased in MPTP treated mice. Lithium treatment alone not only increased striatal $\mathrm{Bcl}-2$ in control mice, but also prevented its reduction as induced by MPTP, while opposing effect was seen with striatal Bax. The neuroprotective action of lithium in this model of Parkinson's disease has been attributed to it induction of $\mathrm{Bcl}-2$ and reduction of $\mathrm{Bax}$.

\section{Co-existence of multiple kinematic internal models}

Zach N.', Kanarek N., Grinvald Y., Inbar D.', Milshtein T. and Vaadia E.'

Physiology Dept., Hadassah medical school, 'ICNC center for neural computation. Hebrew university, Jerusalem

Learning new sensorimotor environments results in formation of an internal model representing the new input-output relationships. An open question is whether there is one internal model or more. When two different environments are learned in succession, the acquisition and consolidation of the internal model may be hindered, suggesting utilization of shared resources while no interference suggests that the brain allows co-existence of the two internal models. Co existence of internal model was shown for kinematics vs. dynamics tasks, but others argued that the confounding parameter is the dependant kinematics variable.

This study examines interference and consolidation to investigate the relationships between two kinematic tasks. We trained subjects to learn a rotational mapping followed by either an opposite rotation or an arbitrary association task, where the target color determines the motor outcome. The next day, subjects were tested for consolidation of rotational mapping. As expected, learning of opposite rotation disrupted consolidation. In contrast, learning arbitrary associations didnt interfere with consolidation of the rotational mapping. This suggests that arbitrary associations and rotational mapping utilize different resources, i.e. internal models.

We then examined the effect of immediate successive learning on acquisition. Learning of the rotational mapping was facilitated when learned in succession of an opposite rotation, suggesting that subjects generalized at the learned parameter level -rotation and providing more evidence that these tasks compete for same resources. Surprisingly, learning rotation task proactively interfered with learning arbitrary associations, suggesting that although they do not interfere for consolidation, they do compete in working memory

These results provide new support for co-existence of kinematics-dependent internal models and raise intriguing questions about the relation between working memory and consolidation.

Enhanced excitability of hippocampal neurons occurs prior to olfactory-discrimination rule learning but is not crucial for its maintenance

Zelcer I ${ }^{1}$ and Barkai $\mathrm{E}^{2}$

'Dept. of Physiology and Zlotowski center for neuroscience. Ben-Curion University, Beer-Sheva: ${ }^{2}$ Center for brain and behavior, Univrtsity of Haifa

We have previously shown that rule learning of an olfactory discrimination task is accompanied by a transient reduction in the post-burst after-hyperpolarization (AHP) and in the neuronal adaptation in piriform cortex pyramidal neurons. The purpose of the present study was to examine whether such training is associated with modifications in intrinsic neuronal properties of CA1 hippocampal neurons and to explore the relations of such modifications in the induction and maintenance of enhanced learning capability.

Water deprived rats were trained in a 4-arm maze to discriminate positive cues in pairs of odors for a water reward. Using intracellular recording in brain slices, we examined the post-burst AHP amplitude in CAl hippocampal neurons at different time intervals after the beginning of training. The averaged value of AHP in neurons from naive rats did not differ from that in neurons from pseudo trained rats, taken 2-7 days after the beginning of training $(3.74+1.15 \mathrm{mV}, \mathrm{n}=17$ in navies compared with $3.73+1.39 \mathrm{mV}, \mathrm{n}=61$ in pseudo trained). Olfactory learning-induced reduction in post-burst AHP in CAl neurons from trained rats was observed as soon as 5 days after the rats began their training (e.g. during the time in which they are still learning the rule) and until one day after rule learning was achieved $(2.52 \pm 0.86 \mathrm{mV}, \mathrm{n}=13$ after five training days, $2.82 \pm 1.20 \mathrm{mV}, \mathrm{n}=17$ one day after rule learning, 6 or 7 days of training for all rats in this time point). At this time window, enhanced neuronal excitability manifested by reduced frequency adaptation was apparent throughout the sampled neuronal population. Once olfactory-discrimination rule learning was established, the AHP amplitude in CA1 neurons resumed its control value $(4.36 \pm 1.42 \mathrm{mV}, \mathrm{n}=8)$, even if training was continued with new odors and enhanced learning capability was maintained.

We suggest that enhanced neuronal excitability in $\mathrm{CAl}$ neurons is strongly correlated with the induction of rule learning of the olfactory-discrimination task. However, once this rule is established, its maintenance is not dependent in intrinsic modifications in these neurons.

ubMunc13-2 acts as a priming factor for large dense-core vesicles and is regulated by calmodulin

Zikich D. ${ }^{1}$, Junge $\mathrm{H}^{2}{ }^{2}$, Brose $\mathrm{N}^{2}$ and Ashery U.

${ }^{1}$ Neurobiochemistry, Tel Aviv University. Israel,

${ }^{2}$ Max-Planck-Institute for Experimental Medicine, Goettingen, Germany

Priming is an essential step in the process of synaptic vesicle maturation that strongly influences vesicle fusion and is mediated by the active zone proteins Munc13-1 and Munc13-2. In chromaffin cells, priming of large dense-core vesicles occurs in a calcium dependent manner and is facilitated by Munc13-1. Structure analyses of the rat Munc13 isoforms have showed the existence of three $C_{2}$ putative calcium-sensor domains and a calmodulin-binding domain (CBD). Thus, the priming activity of Munc13 could be regulated by calcium binding directly to the $\mathrm{C}_{2}$ domains and/or through the interaction with calcium-calmodulin (CaM). Therefore, our aim was to study the role of $\mathrm{CaM}$ binding to Munc13-1/2 in vesicle priming. For that purpose we used the ubMunc13-2, a ubiquitous splice variant of the brain Munc13-2 isoform, and overexpressed it in adrenal chromaffin cells using the Semliki Forest Virus. ubMunc13-2 caused a substantial increase in membrane capacitance in response to flash photolysis of caged calcium. It enhanced significantly the number of primed vesicles prior to flash as well as facilitated vesicle refilling during the 5 -seconds-flash stimulation. In order to elucidate possible interaction with $\mathrm{CaM}$, we mutated the conserved tryplophan residue in the CBD of ubMunc13-2 to arginine (ubMunc 13-2 $2^{\mathrm{W} 38 \%}$ ). Exocytotic response of ubMunc 13-2 ${ }^{\text {w38\% }}$ overexpressing cells was considerably lower compared to wild type protein, although larger than control cells. This suggests that CaM-ubMuncl3-2 interaction facilitates priming but is not a crucial for Munc13-2 activity. In low $\mathrm{Ca}$ pre-flash conditions, both ubMunc13-2 and ubMunc13-2 $2^{\mathrm{W} 387 \mathrm{R}}$ showed reduced priming activity, possibly due to the effects of additional $\mathrm{Ca}^{2+}$ binding site(s) in ubMunc13-2. Thus, we can assume that CaM-ubMunc13-2 interaction has a regulatory nature that facilitates ubMunc 13-2 priming activity following intracellular $\mathrm{Ca}^{2+}$ elevation.

\section{Improving EEG inverse methods using a realistic head} model

Zilberstein A., Kleks A.. Adi-Japha E. and Teicher M.

Bar-llan University, Ramat Gan

Background: The EEG Inverse problem takes an important role in cognitive neuroscience research as well as in clinical applications (c.g. Locating epileptic activities). Several methods have been studied in an attempt to solve this problem (e.g. MNE, BESA, LORETA). Most of the implementations of these methods rely on simplified head models for example: $\mathrm{N}$-layered spherical head model. Some implementations use a realistic head model based on MRI scans, but these are used mostly for taking into account a realistic conductivity in the forward computations

Objective: Improving the current EEG Inverse methods by incorporating realistic structural head information. 
Methods: In previous work we solved the Inverse problem using the approach of Genetic Algorithms. In order to test our work and compare it to other works, we built a simple head model. In the current work we enhanced the head model used in the forward computations to include structural brain information obtained from MRI scans. The forward computation is based on the laws of electromagnetism and Maxwell's equations. Results: We tested our algorithm using simulated EEG data. The dipoles where simulated by averaging 20 cognitive evoked potential trials and the simulated EEG data was generated by using the forward equations on a realistic head model that has a brain-like surface structure. Current head models used by other methods assume a homogeneous spherical head model. We tested data sets with 1-3 simultaneous independent dipole sources, and results showed an improved accuracy in source localization.

Photoreception is required for the onset of the circadian clock in the pineal gland of zebrafish embryo

Ziv L., Toyama R. and Gothilf Y.

Dept. of Zoology, Wise Faculty of Sciences. Tel Aviv University The pineal gland of non-mammalian vertebrates is a photoreceptive organ that contains an intrinsic circadian oscillator. This is indicated by the rhythmic and light-sensitive production of melatonin by pineal glands in culture. Here, we describe the functional development of these features in the zebrafish embryo. A functional circadian oscillator in the pineal gland appears during the second and third day of development as evident by a robust rhythm of serotonin- $N$-acetyltransferase mRNA, characterized by high levels during the night. Since this rhythm is driven by an oscillator, it continues in constant darkness. However, the initiation of this rhythm depends on light exposure during the second day of development, at least 24 hours after fertilization. The effect of light on the circadian
oscillator may be mediated by zebrafish PERIOD2 (ZPER2). a negative component of the molecular oscillator. Zebrafish per2 mRNA expression in the embryonic pineal is light-dependent and remain undetected in embryos that were not exposed to light. Light reception in the 24-hour embryo may be mediated by a number of opsin and cryptochrome photopigments, which are expressed at this stage.

\section{Anticonvulsive treatment of organophosphate induced seizures with nasal midazolam: An electrographic, \\ behavioral and histological Study}

Brandeis R., Kadar T., Levy A., Rabinovitz I., Cohen G., Kapon J.. Tamar G., Sahar R. and Gilat E

Dept. of Pharmacology, IIBR, Ness Ziona

Centrally mediated seizures are common consequence of exposure to organophosphates (OP). These seizures rapidly progress to status epilepticus (SE), and contribute to profound brain injury. To study the clinical and behavioral implications of midazolam as an anticonvulsant, rats were implanted with chronic ECoG electrodes. They were pre treated with pyridostigmine and $\mathrm{HI}-6(0.1$ and $5 \mathrm{mg} / \mathrm{ml}) 20$ min prior to sarin exposure $(1.2 \times L D 50,108 \mu \mathrm{g} / \mathrm{kg})$. Atropine $(2 \mathrm{mg} / \mathrm{kg})$ was administered one-minute post exposure. Within $2-5 \mathrm{~min}$ after sarin exposure animals developed abnormal electrographic discharges that transformed soon after to clectrographic seizure activity (EGSA). Nasal application of midazolam $(1.5 \mathrm{mg} / \mathrm{kg}$ ) activity (EGSA). Nasal application of midazolam ( $1.5 \mathrm{mg} / \mathrm{kg}$ ) after 5 min was found efficient in amelioration of EGSA and
convulsive behavior. Application of midazolam after $30 \mathrm{~min}$ attenuated EGSA in most cases, but was not sufficieni is ameliorate ECoG paradoxical activity and convulsive behavior. Animals that received nasal midazolam at $5 \mathrm{~min}$ EGSA exhibited a moderate decrease in weight $24 \mathrm{hrs}$ post exposure but normal growing curves followed Delaying the treatment to 30 -min EGSA, resulted in severe loss of weight 24 -trs post exposure. These animals returned to original weight only a week later. Cognituve tests utulizing the Niorris Water ilaze, examining iearning and memory processes as weil as reversai learning, demonstrated that nasal midacoiam, auministered ai 5 min EGSA, abolished the effect of sarin intoxication on learning. This treatment, when given 30 min following beginning of convulsions, only decreased the sarin- induced learning impairments. The control group exhibited severe brain lesions in the hipocampus, pyriform cortex and thalamus areas. Nasal midazolam at $5 \mathrm{~min}$ of EGSA, but not at $30 \mathrm{~min}$. prevented the brain damage. In summary, nasal midazolam treatment shortly after initiation of $O P$ induced seizure led to cessation of EGSA and prevented brain lesions and behavioral deficits in the rat model.

Serial memory in monkeys: Changes in balance of mnemonic

strategies as a function of item list lẹngth 'Int. Life Sciences \& Cent. Neural Computation. 'Int. Physics.

Hebrew University, Jerusalem, ${ }^{3}$ Universitî di Roma La Sapienza, Rome

We asked which mnemonic strategies - chaining (item-to-item associations) and/or item ordinal position memory - are naturally preferred for recaling ordered item lists.
We created an experimental paradigm in which monkeys could use cither or both strategies. in working memory and/or long-term memory. 30 abstract images, divided into 10 triplets. were presented cyclically in fixed temporal order. On each trial the monkeys viewed three sequentially presented sample stimuli. followed by a test stimulus consisting of the same 3 images and a distractor image. Their task was to touch the three images in correct order avoiding the distractor. Macaques spontaneously preferred to use long-term memory for recalling image order. The dominant mnemonic routine was retrieval of ordinal number categories. They also used working memory of samples and recall of item-item associations. but only as additional strategies. recruited late in training to avoid distractor images. The categorization phenomenon and balance between chaining and categorization phenomenon and balance between chaining and increased to 4 items. Results indicate that monkeys categorized images of quadruplets according to their ordinal position in each quadruplet, recalling ordinal categories for lists with 4 items as they did for 3 item lists. Categorization was still the dominant strategy in establishing image order in 4-item lists. but its dominance was not as complete as in retrieval of 3 -item lists. Long-term memory associations between individual items contributed significantly not only to avoidance of the distractor but also to the retrieval of image order in the middle part of the list. This suggests that the impact of chaining memory on order retrieval is greater with longer lists.

Supported by a Center of Excellence grant from the ISF and a grant from the National Instimue for Psychobiology in Israel.

Quantitative Analysis of Thalamocortical Synapses in Adult Mouse Barrels

Lev D.L., Weinfeld E. and White E.L

Morphology, Ben Gurion University of the Negev. Beer Sheva

Thalamocortical afferents were labeled by anterogradely transported lysine-fixable biotinylated dextran amine (BDA) injected in vivo into the ventrobasal thalamus. Following light microscopic identification of labeled thalamocortical axons. serial thin sections through the posteromedial barrel subfield werc examined with the electron microscope to assess the spatia distribution of their synapses. Each of two reconstructed axonal segments (78 um and $82 \mathrm{um}$ in length) madel 5 synapses, all asymmetrical. In general, thalamocortical synapses were formed a axonal varicositics or boutons: one synapse occured at a non-varicose region of the axon, i.e. at a preterminal' axonal segment Axonal varicositics formed from I to 3 synapses. Eighty per cent of the thalamocortical svnapses were made with spines. the remainder with dendritic shafts, a proportion consistent with counts obtained from BDA labeled thalamocortical axons in developing barrels (postnatal dav 11), and with those observed for degenerating thalamocortical afferents in the adult

Supported by Israel Science Foundation grant 52/00-3

\section{A Laboratory (Rat) Model for Comparing the} Phenomenological States Induced by Psychoactive Drugs Shraga M., Hen E., Teucher D., and Berger B.D.

Dept of Pychology, Brain \& Behovior Research Center University of Haifa. Haifa

We present an experimental interface using a conditioned taste aversion (CTA) paradigm where laboratory animals provide behavioral information as to whether one particular drug experience is similar or different from another drug experience. Most psychoactive drugs induce conditioned taste aversions (CTA) in laboratory rats when administered after ingestion of novel foods. However, it is not known whether these various drugs produce CTA by the same or different internal states. We explore this question by capitalizing on the well-documented phenomenon that nre-evnociure to a wiven drus will weaken ite notential th nrodice CTA If various drues nröduce CTA bv inducing similar internal states, inen babuuation to the stumuit of one drug should weaken the potential of other drugs to produce CTA. On the other hand, if drugs produce CTA by inducing different internai states, pre-exposure to the stimuli of one drug should not weaken the potential of other drugs to produce CTA. Different groups of laboratory rats were injected daily for 6-days with saline, lithium. or scopolamine. Immediately following 30 - min. sucrose drinking on the pairing day, subgroups were injected with saline, $\mathrm{LiCl}$, or scopolamine. Three days later, the groups were re-tested for sucrose consumption. Ás expected, both $\mathrm{LiCl}$ and scopolamine induced CTA. Also as expected, pre-exposure to $\mathrm{LiCl}_{\mathrm{iCl}}$ or to scopolamine weakened the CTA produced by each of these drugs respectively. However, pre-exposure to $\mathrm{LiCl}$ did not weaken the CTA to scopolamine nor did pre-exposure to scopolamine weaken the CTA to $\mathrm{LiCl}$

These findings suggest that the stimulus properties of $\mathrm{LiCl}$ differ from the stimulus properties of scopolamine, and that the CTA induced by these agents mav be mediated by different mechanisms. In a broader view, these and other findings sugges that drugs may be classified according to their stimulus characteristics - as originally proposed by Overton using a somewhat more cumbersome paradigm. 


\section{Author Index}

\begin{tabular}{|c|c|c|}
\hline Abeles M, 193, 230 & Bello L., 193 & Chen J., 232 \\
\hline Abraham A., 204 & Belmaker RH, $179,195,229$ & Chertkow Y., 190 \\
\hline Abramsky O., 226 & Ben Bashat G., 184 & Chillag-Talmor O., 182,238 \\
\hline Adani R., 221 & Ben Zeev B., 223 & Chorev E., 190 \\
\hline Adi-Japha E., 240 & Ben-Arie N., 201 & Classen J., 214 \\
\hline Admon A., 195 & Benaroya-Milshtein N., 185 & Cohen A., 190 \\
\hline Agam G., $179,195,229$ & Ben-Chaim Y., 184 & Cohen G., 215, 224, 241 \\
\hline Agus S., 211 & Ben-Hur T., 205, 224 & Cohen H, 202,220 \\
\hline Aharon-Peretz J., 179, 229, 236 & Beni SM., 236 & Cohen J.E., 190 \\
\hline Ahissar E., 209 & Benjamin J., 195 & Cohen L., 179 \\
\hline Ahissar M., 182, 219, 224 & Ben-Menachem O., 224 & Cohen M., 179 \\
\hline Akirav I., 179 & Benninger $F ., 184$ & Cohen O., 231 \\
\hline Akkawi S., 216 & Bensafi $M ., 185$ & Cohen $R, 191$ \\
\hline Akselrod S., 220 & Ben-Shachar D., 194, 211 & Cohen Y., 186 \\
\hline Alvarado M., 222 & Ben-Shaul Y., 193, 230 & Cohen-Armon M., 213, 238 \\
\hline Amar S., 179 & Ben-Zur T., 184 & Cohen-Matsliah SI., 191 \\
\hline Amedi A., 225 & Berckovitch Y., 217 & Coorssen J., 197 \\
\hline Amir A., 179 & Berger A., 237 & Cove J., 191 \\
\hline Amit DJ, 185, 191, 219, 222, & Berger B.D., 225, 241 & Crowley J.C., 218 \\
\hline 241 & Bergman $H ., 195,220,223$ & Curti E., 191,219 \\
\hline Amit T., $182,216,225,238$ & Berkowitz L., 213 & Dachir S., 179 \\
\hline Amitai G., 206, 221 & Berman-Shlomovich T., 185 & Dagan Y., 230 \\
\hline Amitai Y., 209 & Bernacchia A., 185 & Dale A.M., 192 \\
\hline Amselem S., 182 & Berry E.M., 199 & Dally Farah R., 191 \\
\hline Andermann M.L., 192 & Bi X., 198 & Dalton E., 229 \\
\hline Angel I., $180,193,198,233$, & Bialer M., 229 & Dar D.E., 182 \\
\hline 234 & Bick A., 185 & Darula S., 223 \\
\hline Anglister L., 193 & Biegon A., $185,222,236$ & Dascal N, 184 \\
\hline Appelbaum L., 180 & Binshtok A.M., 186 & Dayag M., 214 \\
\hline Apter A., 185 & Biran 1., 186 & Dayan Y., 217 \\
\hline Arielly R., I80 & Birk O., 195 & De Vit H., 220 \\
\hline Arien-Zakay H., 180 & Biton I.E., 186 & De Zeeuw C.I., 190 \\
\hline Arraf $Z, 240$ & Blank M., 208 & Decortes L., 192 \\
\hline Ashery U., $189,214,218,220$, & Blank P., 197 & Deouell L.Y., 191 \\
\hline 239,240 & Blatman-Jan G., 193 & Derdikman D., 209 \\
\hline Asraf H., 197 & Blau H., 198 & Desmedt A., 192, 233 \\
\hline Assaf Y., 180, 186 & Bledi Y., 188 & D'Esposito M., 191 \\
\hline Atlas D., $181,191,201,227$ & Blinder P., 186 & Devir A., 189 \\
\hline Attali B., 230 & Blivis D., 187 & Devor A., 192 \\
\hline Avidan H., 181, 209, 214, 226 & Bloch-Shilderman E., 187 & Devor M., 234 \\
\hline Avidor B., 182,217 & Blum B., 187 & Di Segni A., 192 \\
\hline Avissar S., 217 & Blumenfeld A., 237 & Dina C., 195 \\
\hline Avital A., 181 & Boas D.A., 192 & Ding D., 229 \\
\hline Avraham Y., 199 & Bock J., 187 & Diskin T., 192 \\
\hline Axelrod F, 237 & Bogoch Y., 188, 236 & Dolev I., 192,222 \\
\hline Ayali A., 183 & Boraud T., 220,223 & Dong H., 196 \\
\hline Azmon B., 212 & Boutboul O., 188 & Dorfman N., 181 \\
\hline Azouz R., 181 & Bower J.M., 192 & Dori A., 190 \\
\hline Bachner-Melman R, 195 & Bracha A., 188 & Dreier J.P., 198, 215, 228 \\
\hline Bahar A., 181 & Brandeis R., 188, 224, 241 & Dremencov E., 193 \\
\hline Bakalash S., 226 & Braun E., 233 & Drori R., 193,230 \\
\hline Balass M., 181 & Braun K., 187, 197, 204 & Dudai Y., 179, 181, 194, 199, \\
\hline Banai K., 182 & Braw Y., 215 & 209,233 \\
\hline Banitt Y., 182 & Brenneman DE., 199 & Dunn A.K., 192 \\
\hline Bar-Am O., 182, 238 & Brenner T., 203, 206, 216, 221 & Durrant A.R., 193 \\
\hline Baranes D., 186,191 & Breznitz N., 203 & Duvdevani R., 193 \\
\hline Bar-Hen H., 238 & Breznitz Z., 188, 189, 203, 218 & Dvir E., 193 \\
\hline Barhum Y, 184, 201, 214 & Brosch M., 218 & Ebstein RP, 195 \\
\hline Bar-Ilan A., 182 & Brose N., 240 & Echarte M.M., 194 \\
\hline Bar-Joseph A., 182, 217 & Brosh I., 189 & Edelheit S., 194 \\
\hline Barkai E., 189, 191, 202, 220, & Bröstle O., 184 & Egoz I., 188 \\
\hline 240 & Bruns D., 239 & Ehrenstein W.H., 238 \\
\hline Barkai G., 210 & Bulvik S., 184, 214 & Eick I., 194 \\
\hline Barkan S., 183 & Burlingame A., 223 & Eilon K., 196 \\
\hline Barlasov A., 183 & Burshtein A., 184, 214 & Einav S., 233 \\
\hline Barnea A., 183 & Burstyn-Cohen T., 206 & Einevoll G.T., 192 \\
\hline Barnea A., 183, 205 & Buskila E., 189 & Eisenberg M., 194 \\
\hline Baron A., 215 & Buskila Y., 209 & Elbaz J, 189 \\
\hline Bar-Yosef O., 196 & Butovsky E., 189 & Elgazar V., 221 \\
\hline BazBarChani M., 205 & Butovsky O., 181,189 & Elhadad (Rosolio) N., 195 \\
\hline Beck H., 184, 229, 232, 237 & Cahalon L., 226 & Elkobi A., 184, 195 \\
\hline Becker A., 232 & Calandreau L., 192 & Engel A.K., 195 \\
\hline Bejar C., 231 & Caspi L., 189 & Engelstein R., 195 \\
\hline Belelovsky K., 184, 207 & Chapman J., 208 & Engler G., 195 \\
\hline
\end{tabular}


Erez. H., 196

Erlich S.. 192.196

Ezra D., 198

Fainzilber M., 204. 223

Farchi N.. 196

Farkas D. 196

Feinstein N., 197

Feldon J., 2 I3

Fendyur A., 197

Ferdman N., 197

Ferster D. 211

Fink G., 182, 217

Fink N., 199

Finkelstein E.. 234

Fiorito G. 234

Fishbein I., 197

Fishbine E. 179

Flash T., 217, 232, 234, 239

Fleidervish I.A.. 186, 207, 214

Fleisher-Berkovich S.. 197

Fraifeld V., 197.227, 239

Fride E. 198

Friedman A., 190, 198, 201. $206,215,228$

Fricdman J.E.. 180, 193, 198 233.234

Frisch A.. 210

Fritschy J.M.. 197

Fromovitz M., 198

Fry P., 185

Fuchs S., 223

Fuhrmann G.. 204

Furman S., 199

Furst E., 199

Gabbay E., 199

Gabbay H., 199

Gabizon R., 195, 205, 207, 217

Gak E., 200, 210, 223

Gal A., 186

Gal R., 200

Galili M., 208

Galski H. 237

Ganzburg V., 199

Gefen-Aricha R., 193

Gembom E., 211

Gershoni-Baruch R., 179

Gersner R., 228

Gerstberger R., 235

Geylis V., 226

Gidon A.A., 200

Gilad G.M., 201

Gil-Ad I., 200, 214

Gilad V.H., 201

Gilat E., 187, 224, 241

Gileadi C., 233

Gilgun-Sherki Y., 201, 227

Gilon C., 212

Goelman G., 185, 216, 225

Golan H., 201, 208, 212, 213

Golan-Lev T., 201

Goldman B, 223

Goldsher Y. D., 229

Goldshmit Y., 196

Golomb D., 202

Goncharov I., 189

Gonzalez-Dunia D., 223

Goshen I., 238

Gothilf Y., 180, 241

Gores I., 199

Graucr E., 202, 211

Grcenberg I., 202

Greenberg M.L., 229

Greig N.H., 195

Grigoriadis N., 195, 206, 207, 216

Grinfeld A., 202

Grinvald Y., 240
Gritsenko I., 195

Grossberger T., 202

Grothe B., 228

Grundland A., 189

Gur M., 202

Gurevich A., 203

Gurshumov N., 203

Gutman H., 179

Gutman M., 218

Gutnick M.J., 186, 207, 214

Guy U., 203

Hadad B., 203

Hagallili Y., 239

Hai A., 203

Haim H., 233

Haimov I., 203

Halimi M., 217

Hallak M., 208, 212

Hamra Y., 203

Hanrahan S., 222

Hanuka E., 203

Hanz S. 204, 223

Harel M. 212, 219

Hari R., 219

Haring R. 233

Haroush K., 222

Harriton-Gazall E., 212

Hart R.P., 228

Harwood A.J., 229

Haspel G., 200

Hasson U., 204, 220

Hauben E., 220

Hauptmann B., 232

Hauser JM., 199

Hecht D. 204

Heinemann U., 198, 206

Heinemann W., 228

Heller A., 191

Helmeke C., 204

Helmstaedter M., 210

Hen E., 24I

Hendler T., 180, 212, 219

Herman-Gispan I., 193

Hershfinkel M., 180, 221

Hershler O., 204

Higazi AE. 216

Hijazi N., 205

Hill JM., 199

Hirschmann S., 205

Hochner B., 196, 217, 234, 239

Hochstcin S., 183, 185, 194, $204,221,222,230,241$

Hoffman K.B. 198

Hollander N., 185

Horowitz M., 230, 235

Huerta J.J., 204

Huleihel M., 208, 212

Hurwitz I., 208, 234

Ilan Y., 199

Ilin Y., 205

Inbar D., 240

Inberg A., 205

IronyTur-Sinai M., 206

Irony-Tur-Sinai M., 216

Israeli, 187

Ivens S., 206

Jacobson S., 206

Jaffard R., 192

Jiang H., 235, 237

Joel D., 228

Jones B.C., 179

Jones S.R. 192

Jongen-Relo A.L., 213

Jovin T.M., 194

Juknat A., 189

Junge H., 240

Kadar T., 179, 187, 241
Kagan H.M., 201

Kaiserman I., 197

Kalcheim C., 206

Kalef E., 181

Kalmanzon E.. 226

Kamber D., 207

Kamsler A., 207

Kanarek N., 240

Kaphzan H., 195, 207

Kaplanski J., 197

Kapon J., 202, 241

Karasik Y., 233

Kariv-Inbal Z., 207

Karni A., 232

Karry R., 194, 211

Kasher C., 207

Kashtutsky I., 208

Katchalski-Katzir E., 181

Katz L.C., 218

Katzav A., 208

Katzoff A., 208, 213

Kavushansky A., 209

Kesner Y., 215

Khair A., 191

Khan R., 185

Kimhi S., 209

Kinor N., 193

Kipnis J., 181, 209, 214

Klar A, 203

Klein E., 194, 211

Klein R., 238

Kleinfeld D., 202

Kleks A., 240

Kloog Y., 216

Kluger M.J., 197

Knight R.T., 191

Knutsen P.M., 209

Kobilo T., 209

Kodama T., 215

Koester J., 234

Kofman O., 184

Kogan I., 210

Koh J.Y., 193, 198

Kohen R., 203, 235, 239

Kohler M., 204

Koltzenburg M., 204

Korczyn A.D., 208

Korkotian E., 210

Korn A., 201

Korngreen A., 210

Koronyo-Hamaoui M., 200, 210

Kovacs I., 194

Kovalev E., 231

Kozak A., 180, 193, 198, 233 234

Kozak W., 197

Krakovsky M., 234

Krieglstein K., 233

Kuulmann S., 211

Laifenfeld D., 211

Lamensdorf I., 211

Lamm O., 218

Lamp I., 211

Lamschtein C., 205

Las-Ahdut L., 196, 212, 237

Lazarovici P., 212, 235, 237

Lecht S., 212

Lee J.Y., 193, 198

Leiba H., 212

Lerner Y., 212

Leshem M., 188, 191, 197,

$$
\text { 204, } 238
$$

Lev D.L., 241

Lev V., 212

Levav T. 213

Levavi-Sivan B., 214 
Levi O.. 213

Levidor L., 215

Levin N., 213

Levine J., 189

Levine R.A., 235

Levitan D. 213

Levkovitz Y., 214

Lev-Tov A., 187, 199, 233

Levy A.. 187, 24

Levy I., 204, 220

Levy Y., 217

Levy Y.S., 184,214

Lewitus G.M., 214

Libersat F., 200, 226

Libman L., 214

Lider O., 226

Linial M., 188, 205, 236

Listowsky I., 197

Litvak V., 214

Loewenstein A., 212

London M., 182

Lu W-Y. 196

Luques L., 215

Lynch G. 198

Maavan R., 215

Mador N., 233

Magen I., 215

Maggio N.. 197

Major S., 215

Makranz C., 215

Malach R., 204. 212, 219. 220

Malkesman O.. 215

Malkinson G., 216, 232

Mandel S., 216, 225, 227

Manor Y., 190, 202

Maravi- Pinto FY., 216

Marciano D., 216

Martin K.A.C., 182

Massarwa R. 204. 223

Matti U.. 239

Matuzany-Ruban A., 217

Matzner H., 217

Mayer-Sonnenfeld T. 217

Mayk A., 186

McKvton A., 217

Mechoulam R., 199

Medzihradsky K.. 223

Mcilin S. 182, 217

Meiner M. 226

Meirav T.. 233

Meiri N. 194

Mclamed E., 184, 201, 214. 227

Mclamed 1., 201

Melamed R, 239

Melikyan A. 218

Merbl Y., 202

Meshulam H., 22I

Mezer A. 218

Michaeison DM. 189, 192, 213.222

Miller N., 208

Miller-Shaul S., 189, 218

Milman A., 218

Milshtein T. 240

Mishal A., 183

Misrahi A., 218

Modai I., 198

Moisesco-Yiflach T., 218

Moll C.K.E., 195

Molshanski-mor S.. 220

Mongillo G., 191, 219

Morales C.R., 19

Moran A.. 180

Moran D.S., 219

Mordechav S., 209, 214, 228

Mukamel R., 219
Munno D., 223

Naama D., 197

Naaman S., 187

Nachliel E., 218

Nahshon 1., 198

Nahum M. 219

Naimark A., 220

Nairn A.C., 184

Naijar S., 217

Narayanan S.N., 192

Nathan C., 220, 223

Neeman M, 220

Nelken I. 196, 212, 237

Nevo I., 189

Nevo U., 220

Nili U., 220

Nir Y., 204, 220

Nitzan Y.B., 22

Nizan A., 221

Nizri E., 221

Nottebohm F., 183

Nudelman A., 200

Oddoux C., 237

Ofek E., 22

Offen D., 184, 201, 214, 227

Okun A., 199

Ophir G., 222

Oren R., 189

Orlov T., 185, 222, 241

Osher Y., 195

Ovadia $\mathrm{H}_{\text {., }} 195$

Overstreet D.H., 193, 215

Paden C., 185

Panet A., 233

Pareto D., 222

Parnas H., 184, 228

Parnas I., 184, 197, 228

Pascual-Marqui R., 201

Paul L 197

Pavlovskaya M., 222

Paz R., 220, 223

Peled A., 198, 232

Peretz A., 230

Perez-Reyes E., 237

Peri D., 188

Perl O. 223

Perlson E., 204, 223

Petel-Galil Y., 223

Peters B.. 233

Pianka P., 180, 212

Pick C.G., 185, 208, 218

Pietr M. 209

Pietrasanta L.I.. 194

Pinkas-Kramarski R., 192, 196

Pollak Y., 224

Poltyrev T., 215

Polyak M.. 193

Portnoy F., 188

Prager-Khoutorsky M., 224

Pratt H., 214, 218, 221

Premkumar A., 211

Putter-Katz H., 22

Rabey J.M., 201

Rabinovitz I., 224, 241

Rabinowitch I.. 224

Rado R.. 183

Rahamimoff R., 197

RassisA., 183

Raveh A., 197

Ravch L., 224

Raz N., 225

Raz S., 193, 225

Rcich R., 212

Reichert F., 215

Remy S., 229

Renvall H. 219

Rephaeli A., 200
Rettig J., 239

Reuveny E., 226

Reznichenko L., 216, 225

Rich R., 225

Richstein A., 182, 217

Richter-Levin G., 181, 202 $205,206,209,210,236$

Ringel I., 212

Rinski L., 203

Riven I., 226

Rolls A., 226

Rosenbaum H., 179

Rosenberg L.A., 226

Rosenblum K., 184, 189, 191, $195,207,211,237$

Rosenmann H. 205, 226

Roses A.D., 213

Rotshenker S., 215

Rotshtein P., 180

Saar T, 213

Sadaka Y., 226

Sadan O., 227

Sagi D., 229

Sagi O., 197, 227

Sagi Y., 227

Sagiv N, 227

Sahar R., 179, 241

Sakmann B., 210, 227

Salpeter M.M., 193

Sapiens N., 188

Sarid L., 227

Sarig O., 202

Schaefer A.T., 210

Schaefer E., 235

Schatz G., 233, 234

Schaub C. 229

Scheffler B., 184

Scheich H., 218

Schiller J., 209

Schönberg T, 228

Schori H., 181, 226

Schottelndreier H., 230

Schramm A., 214

Schreiber G., 217

Schwartz JH., 213

Schwartz M., 181, 189, 209 $214,220,226,228$

Schwimmer H., 235

Segal M., 197, 207, 210

Segel L.A., 228

Seger R., 238

Segev I., 182, 200, 224, 227

Segev L., 226

Seidl A.H., 228

Seiffert E., 198, 228

Sekler I., 180, 221

Sela R., 228

Shabat-Simon M., 215

Shaked G.M., 207

Shaked I., 228

Shaked M., 223

Shaked M.G., 195

Shaked Y., 205

Shalem Y., 179

Shalev E., 191

Shaltiel G., 229

Shamay-Tsoory S.G., 229

Shamgar L., 230

Shamir A., 229

Shani R., 229

Shapira E., 231

Shapiro I., 180, 193

Shapiro J., 229

Shappir J., 190

Shappir Y, 203

Shavit Y., 238

Shefer O., 203 
Shein N.A., 230

Shmiel O., 230

Shmiel T., 230

Shneor E., 221, 230

Shoenfeld Y., 208

Shoham S., 215, 231

Shohami E., 192, 196, 203, $216,230,236,239$

Shraga M., 241

Shtoyerman E., 218

Sicsic C., 203

Sidorik L., 239

Sigal M., 180

Silver H., 190, 231

Silverman W.F., 221

Simantov R., 211

Simmons D.A., 198

Slonimsky G., 231

Smorodinski NI., 222

Sobel N., 185, 231

Sochivko D., 232

Sohl G., 190

Sonego H., 224

Soreq H., 190, 196, 224, 231

Sorkin A., 232

Sorokin Y., 208, 212

Sosnik R., 232

Soteropoulos P., 228

Spira M.E., 190, 196, 203, 207, $216,224,231,232$

Spira T., 203

Spittau B., 233

Spitzer H., 233

Stanleigh J., 206

Stefan K., 214

Stehberg J., 233

Steiner I., 233

Steinitz M., 226

Sterin-Cohen M., 212

Stern E.A., 212

Stern S., 238

Stien R., 192

Stolovitch C., 212

Strauss I., 233

Striem S., 233, 234

Stroomza M., 227

Strous R.D., 223

Sukhotinsky I., 234

Sumbre G., 217, 234

Susswein A.J., 208, 213, 234

Susswein Y.Z., 208

Syed N., 223
Tabakman R., 212, 235

Tal L., 216

Tal-Or P., 192

Talpalar A.E., 189

Tamar G., 241

Tamir I., 235

Tayar S., 188, 236

Tchaikovskaya T., 197

Teicher M., 230, 240

Teucher D., 241

Tibi Y., 229

Tochros D., 228

Tolias P., 228

Tomer R., 229, 236

Tonnen R., 220

Touati D., 215.

Tour O., 184

Toyama R., 241

Tsenter J., 236

Tsitrinbaum Z., 236

Tsoory M. M., 236

Tucker K.L., 184

Turetz J., 188

Twiss J.L., 204

Tzur G., 237

Uebachs M., 237

Ulanovsky N., 196, 237

Ulbert I., 192

Umansky-Safanova S., 237

Vaadia E., 220, 223, 240

Vais O., 239

van-Minnen J., 204

Vardinon-Friedman H., 237

Verhage M., 220

Visochek L., 213, 238

Vogel Z., 189

Vulih I., 199

Wagner M., 238

Wang Z., 233

Weiner I., 200

Weinfeld E., 226, 241

Weinreb O., 182, 190, 238

Weinshall D., 232

Weinstock M., 188, 215, 231

Weissman B.A., 224

Weizman A., 200, 210, 214

Weizman R., 218

Weizman Y., 193

Weksler A., 182, 217

Weller A., 215

Wernig M., 184

White E.L, 241
White E.L., 226

Wilf A., 185

Willecke K., 190

Willis D., 204

Wolf G., 238

Wolfson M., 197, 227, 239

Wormser U., 185

Xiao X., 228

Yaari Y., 232, 235, 238

Yacovan A., 182, 217

Yadid G., 193, 215

Yakovlev V., 185, $24 \mathrm{I}$

Yaniv I., 185

Yao Z., 238

Yarom Y., 190, 227

Yativ M., 238

Yatsiv I., 236

Yavelsky V., 239

Yekutieli Y., 239

Yeshurun H., 220

Yeshurun-Cohen A., 239

Yinon U., 203

Yirmiya R., 224, 231, 238

Yisraeli E., 199

Yitzhaiek S., 190, 203

Yizhar O., 239

Yogev-Falach M., 182

Yonas A., 191

Youdim MBH., 182, 190, 216, $225,227,238,240$

Younis F., 193

Yue C., 238

Zaaroor M., 214

Zach G., 188

Zach N., 240

Zaidel E., 183, 205

Zakai H., 207

Zangen A., 189

Zelcer I., 240

Zeldich E., 214

Zheng J-O., 204

Zikich D., 240

Zilberman I., 199

Zilberstein A., 240

Zimmerberg J., 197

Ziv L., 241

Ziv T., 195

Zohar A.H., 195

Zohary E., 213, 217, 222, 225 241

Zolotarev O., 199

Zuckerman L., 200 

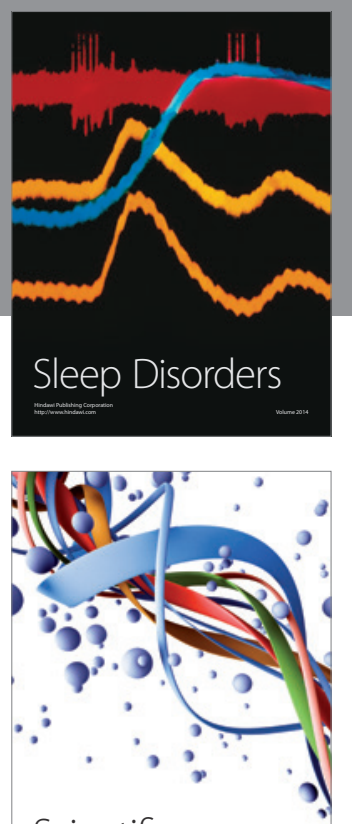

Scientifica
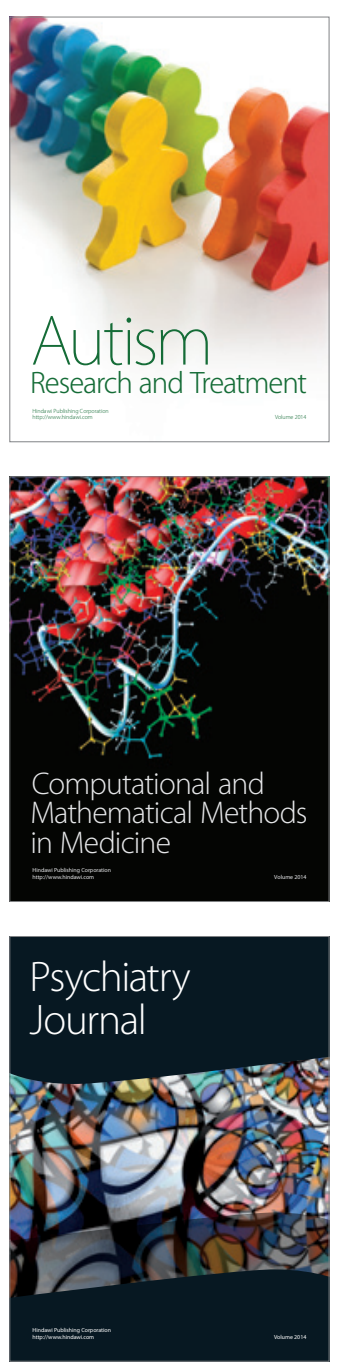
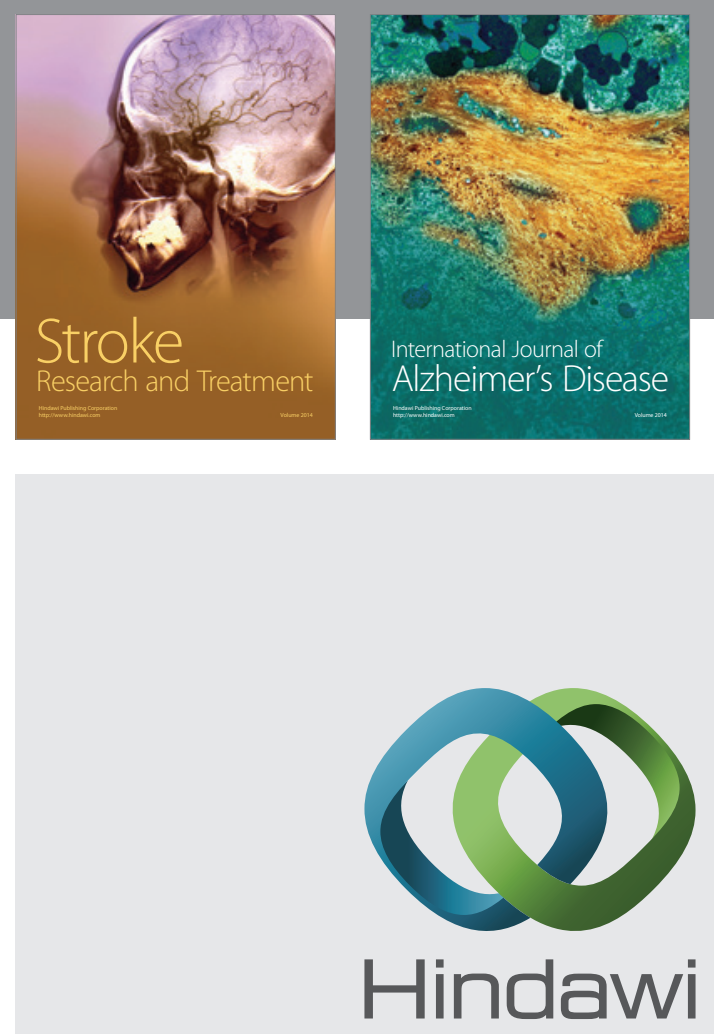

Submit your manuscripts at

http://www.hindawi.com
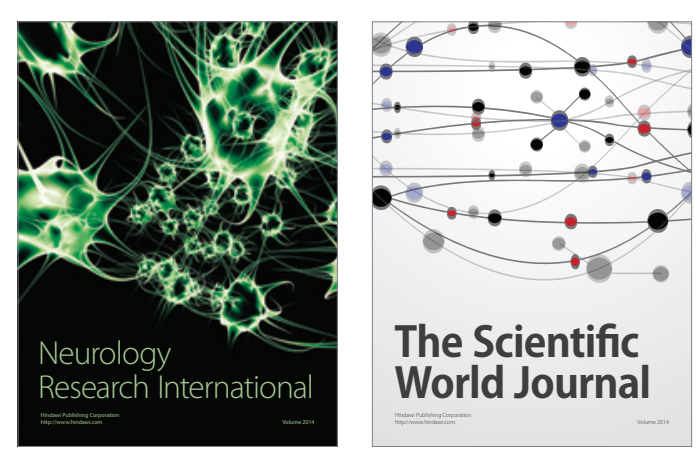

The Scientific World Journal

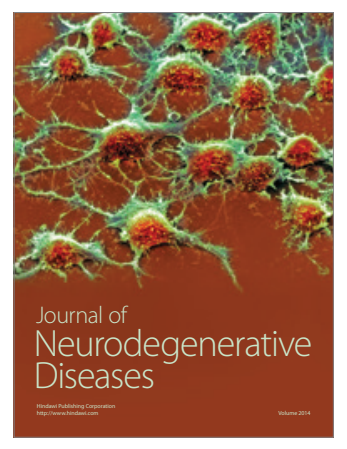

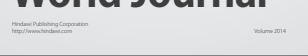

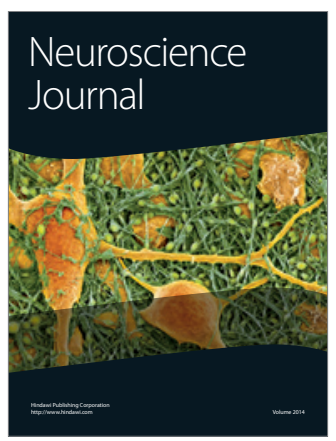

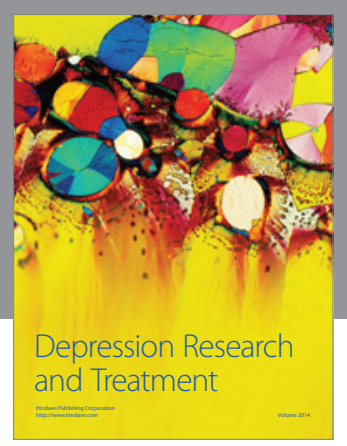
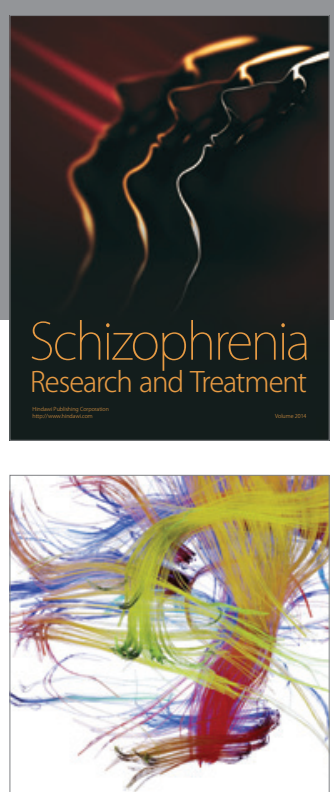

Brain Science

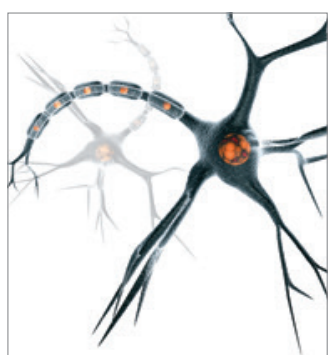

Neural Plasticity
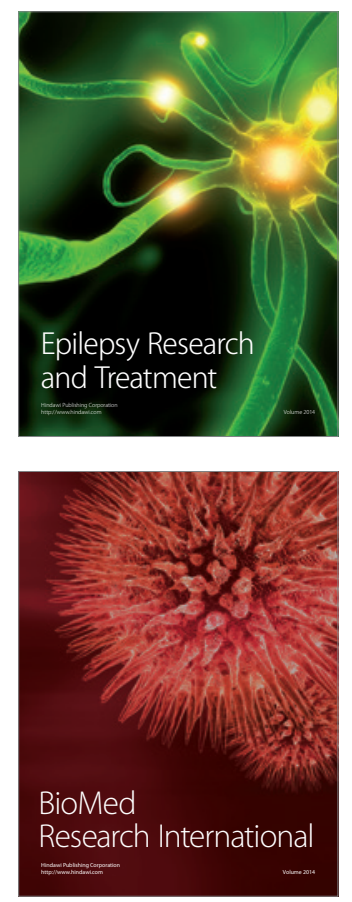

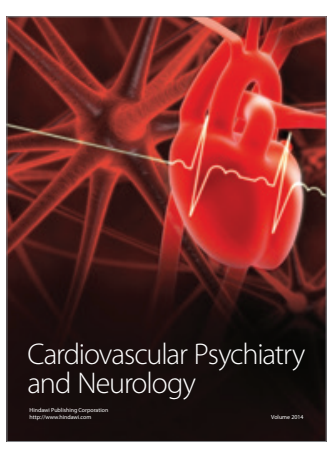

Parkinson's

Disease
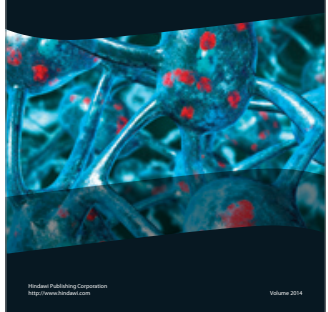